\title{
Homological manifestations of quantum group duality
}

\author{
by \\ Jason Crann \\ A thesis submitted to the \\ Faculty of Graduate and Postdoctoral Affairs \\ Carleton University \\ and \\ l'École Doctorale Sciences Pour l'Ingénieur \\ Université Lille 1: Sciences et Technologies \\ in partial fulfillment of the requirements for the degree of
}

Doctor of Philosophy

in

Pure Mathematics

(C) Copyright

2015, Jason Crann

September 9, 2015 



\section{Addresses}

School of Mathematics \& Statistics

Carleton University

4302 Herzberg Laboratories

1125 Colonel By Drive

Ottawa, Ontario, K1S 5B6

\&

Laboratoire Paul Painlevé

Université Lille 1

Cité Scientifique

218 Bâtiment M2

59655 Villeneuve d'Ascq Cedex 


\section{Abstract}

The connection between amenability of a locally compact group $G$ and injectivity of its group von Neumann algebra $\mathcal{L}(G)$ has been studied by many of the world's leading operator algebraists for decades. In this work we clarify this connection by showing the equivalence between amenability of $G$ and 1-injectivity of $\mathcal{L}(G)$ as an operator module of the Fourier algebra $A(G)$. In fact, we prove a corresponding result for all locally compact quantum groups $\mathbb{G}$, establishing at the same time new homological manifestations of quantum group duality, and a novel tool for the development of abstract harmonic analysis on locally compact quantum groups. We give several applications of our general duality result, including a proof that closed quantum subgroups of amenable quantum groups are amenable, and a simplified proof that co-amenability and amenability of the dual are equivalent for compact quantum groups which avoids the use of modular theory, suggesting a potential avenue for generalization beyond the compact setting. We also introduce a notion of inner amenability for locally compact quantum groups and study its connection to relative injectivity, establishing further homological manifestations of duality which not only help to elucidate previously known results, but provide new approaches to open problems concerning the operator homology of $A(G)$.

Key words: abstract harmonic analysis, locally compact quantum groups, amenability, operator algebras, operator modules. 


\section{Résumé}

La connexion entre moyennabilité d'un groupe localement compact $G$ et l'injectivité de son algèbre de von Neumann $\mathcal{L}(G)$ a été étudié depuis des décennies, et représente l'une des connexions les plus importantes entre l'analyse harmonique et les algèbres d'opérateurs. Dans ce travail, nous clarifions cette connexion en montrant l'équivalence de moyennabilité de $G$ et l'injectivité de $\mathcal{L}(G)$ en tant que module d'opéateur sur l'algèbre de Fourier $A(G)$. En fait, nous établissons le résultat correspondant au niveau des groupes quantiques localement compacts $\mathbb{G}$, fournissant à la fois des nouvelles manifestations de la dualité des groupes quantiques, et un nouvel outil pour le développement de l'analyse harmonique abstraite sur les groupes quantiques localement compacts. En appliquant le résultat principal, nous montrons qu'un sous-groupe quantique fermer d'un groupe quantique moyennable est moyennable, et nous prouvons un théorème de décomposition pour les applications de module complètement bornées.

Un des plus grands problèmes ouverts dans l'analyse harmonique abstraite sur les groupes quantiques localement compacts est la dualité entre moyennabilité et comoyennabilité. Le résultat le plus général jusqu'à présent est dans le cadre compact, disant qu'un groupe quantique compact $\mathbb{G}$ est co-moyennable si et seulement si son

dual $\widehat{\mathbb{G}}$ est moyennable. La preuve est extrêmement technique, utilisant la théorie modulaire des algèbres de von Neumann d'une manière essentielle [104]. Pour notre application finale du résultat principal, nous donnons une preuve simplifiée de cette théorème qui évite la théorie modulaire, suggérant une technique potentielle pour la 
généralisation au-delà du cadre compact.

Nous introduisons également une notion de moyennabilité intérieure pour les groupes quantiques localement compacts et nous étudions sa connexion à l'injectivité relative. En général, moyennabilité intérieure de $\mathbb{G}$ entraîne l'injectivité relative de $L^{\infty}(\widehat{\mathbb{G}})$ en tant que module d'opérateur sur $L^{1}(\widehat{\mathbb{G}})$, et l'inverse est vrai dans le cadre d'un groupe, c'est-à-dire, un groupe localement compact $G$ est intérieurement moyennable si et seulement si $\mathcal{L}(G)$ est relativement injective sur l'algèbre de Fourier $A(G)$. La démonstration utilise une nouvelle caractérisation des groupes moyennables intérieures, qui, incidemment, répond à une question de Lau et Paterson [67]. Nos techniques nous permettent également de résoudre partiellement une question de Forrest, Lee et Samei concernant la projectivité relative d'opérateur de $A(G)[33, \S 4$, ainsi que le problème ouverte du platitude relative d'opérateur de $A(G)$ [2]. Nous terminons en montrant l'auto dualité de platitude des algèbres de convolution des groupes quantiques localement compacts.

Mots clés: analyse harmonique abstraite, groupes quantiques localement compacts, moyennabilité, algèbres d'opérateurs, modules d'opérateurs. 


\section{Acknowledgements}

First and foremost I would like to thank my supervisors, Matthias Neufang and Mostafa Mbekhta, for providing guidance and support throughout the project, and for giving me the opportunity to study in Lille. The year in France was full of adventure, both within and without mathematics, and I am grateful for having had the experience. A special thanks also goes to Flunch $₫$, whose unlimited supply of french fries helped fuel the project in its early stages.

I would like to thank the following colleagues for helpful discussions at various points over the past four years: Mahmood Alaghmandan, Yemon Choi, H. Garth Dales, Matthew Daws, Patrick Farrell, Brian Forrest, Mehrdad Kalantar, Matthew Kennedy, Rupert Levene, Emmanuel Lorin, David Kribs, Sutanu Roy, Zhong-Jin Ruan, Nico Spronk, Zsolt Tanko, Ivan Todorov, and Roland Vergnioux. I would also like to thank my committee members Yuly Billig, Prosenjit Bose, Benoît Collins, Brian Forrest, Emmanuel Fricain and Anthony Lau.

Thank you to my friends and family for their support and their encouragement to step down from the clouds every once in a while. Special thanks to the Whitby crew, the Guelph crew and the Ottawa crew: I hope our random encounters in the beautiful places of the world continue.

Behind every man lies a great woman. It goes without saying that this thesis would not have been possible without the continual support and understanding of my better half, Carley. 


\section{Contents}

Addresses $\quad$ ii

Abstract $\quad$ iii

Résumé $\quad$ iv

Acknowledgements $\quad$ vi

1 Introduction 1

2 Preliminaries $\quad 5$

2.1 Locally Compact Groups . . . . . . . . . . . . . . . . . . . 6

2.2 Banach Algebras and Banach Modules . . . . . . . . . . . . . . 8

2.3 von Neumann Algebras . . . . . . . . . . . . . . . . . . . . 14

2.4 Operator Spaces . . . . . . . . . . . . . . . . 20

2.5 Operator Modules and Homology . . . . . . . . . . . . . 25

3 Locally Compact Quantum Groups 30

3.1 von Neumann Algebraic Quantum Groups . . . . . . . . . . . . . . 30

3.2 The Fundamental Unitary . . . . . . . . . . . . . . . . . . 35

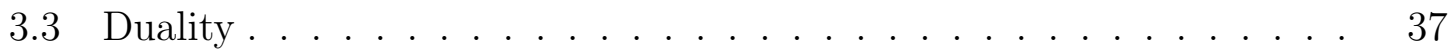

3.4 The Antipode . . . . . . . . . . . . . . . . . . . 40

3.5 Quantum Groups in the Universal Setting . . . . . . . . . . . . 43

3.6 Compact Quantum Groups . . . . . . . . . . . . . . . . . 47 
3.7 Completely Bounded Multipliers . . . . . . . . . . . . . . . . 49

4 Amenability and Injectivity 53

$4.1 \mathcal{T}\left(L^{2}(\mathbb{G})\right) \ldots \ldots \ldots \ldots \ldots \ldots \ldots$

4.2 Covariant Conditional Expectations . . . . . . . . . . 65

4.3 Injective Quantum Group Modules . . . . . . . . . . . . 75

4.4 Applications . . . . . . . . . . . . . . . . . . 91

5 Inner Amenability and Relative Injectivity 107

5.1 Classical Theory . . . . . . . . . . . . . . . . 107

5.2 Quantum Theory ... . . . . . . . . . . . . . 114

5.2.1 Definition and Basic Properties . . . . . . . . . 115

5.2 .2 Relative Injectivity . . . . . . . . . . . . . . . . . 124

5.2.3 Biflatness and Biprojectivity . . . . . . . . . . . 127

6 Future Directions $\quad 134$

$\begin{array}{ll}\text { Bibliography } & 136\end{array}$

$\begin{array}{ll}\text { Index } & 144\end{array}$ 


\section{Chapter 1}

\section{Introduction}

One of the primary objectives of abstract harmonic analysis is to study the intrinsic structure of locally compact groups through their manifestations at the level of Banach and operator algebras. In fact, the rapidly developing field of abstract harmonic analysis on locally compact quantum groups has been evolving precisely in this spirit. Moreover, this correspondence has produced many of the historical examples of operator algebras over the years, and has been an indispensable tool in studying their underlying structure. One of the most important connections in this context is the equivalence of amenability of a discrete group $G$ and injectivity of its von Neumann algebra $\mathcal{L}(G)[15,28,65]$.

More generally, amenability of a locally compact group $G$ always entails the injectivity of $\mathcal{L}(G)$ [39]. The converse is not true in general, however. Indeed, a result of Connes' [15, Corollary 7], attributed to Dixmier, states that $\mathcal{L}(G)$ is injective for any separable connected locally compact group, e.g., $G=S L(n, \mathbb{R})$ for $n \geq 2$. In order to find a strengthening of injectivity which would be equivalent to amenability, there have been two main approaches: in terms of additional properties of the underlying group, or of the associated conditional expectations. In the spirit of the first approach, Lau and Paterson showed that $G$ is amenable if and only if $\mathcal{L}(G)$ is injective and $G$ is inner amenable [66, Corollary 3.2]. Following the second approach, 
Soltan and Viselter recently proved, in the more general setting of locally compact quantum groups $\mathbb{G}$, that amenability is equivalent to the existence of a conditional expectation $E: \mathcal{B}\left(L^{2}(\mathbb{G})\right) \rightarrow L^{\infty}(\widehat{\mathbb{G}})$ which maps $L^{\infty}(\mathbb{G})$ into the center of $L^{\infty}(\widehat{\mathbb{G}})$ $[95$, Theorem 3], where $\widehat{\mathbb{G}}$ denotes the dual of $\mathbb{G}$. In this thesis we provide a homological perspective of this connection at the level of locally compact quantum groups by presenting new characterizations of amenability using the $L^{1}(\widehat{\mathbb{G}})$-module structure of $L^{\infty}(\widehat{\mathbb{G}})$ and the $\mathcal{T}\left(L^{2}(\mathbb{G})\right)$-module structure of $\mathcal{B}\left(L^{2}(\mathbb{G})\right)$, leading us to the concept of covariant injectivity. This new perspective not only clarifies the above connection between amenability and injectivity of locally compact groups, it generates important homological manifestations of quantum group duality and provides a novel tool for the development of abstract harmonic analysis on locally compact quantum groups an extremely active field of research.

The thesis is structured as follows. We begin in chapter 2 with a review of the mathematical essentials from abstract harmonic analysis, operator algebras, operator spaces and the homology of operator modules. Chapter 3 is devoted to a brief introduction to locally compact quantum groups in the sense of Kustermans and Vaes [64]. We present the main constructions and results concerning fundamental unitaries, non-abelian Pontrjagin duality and completely bounded multipliers.

Chapter 4 contains the main results of the thesis, a portion of which has been accepted for publication in the Transactions of the American Mathematical Society [17]. We begin with an overview of the various Banach algebra structures on $\mathcal{T}\left(L^{2}(\mathbb{G})\right.$ ), introduced and studied in $[74,49]$. In particular, we highlight the duality of the products arising from $\mathbb{G}$ and its dual $\widehat{\mathbb{G}}[56]$, which is of fundamental importance for the thesis. We then study the existence of conditional expectations $E: \mathcal{B}\left(L^{2}(\mathbb{G})\right) \rightarrow L^{\infty}(\widehat{\mathbb{G}})$ which commute with the various $\mathcal{T}\left(L^{2}(\mathbb{G})\right)$-module structures on $\mathcal{B}\left(L^{2}(\mathbb{G})\right)$. This yields new characterizations of amenability, co-commutativity, compactness and injectivity.

In the section on injective quantum group modules we establish the equivalence 
of amenability of $\mathbb{G}$ and 1-injectivity of $L^{\infty}(\widehat{\mathbb{G}})$ in the category of operator $L^{1}(\widehat{\mathbb{G}})$ modules. In the group setting, the dual of the commutative quantum group $L^{\infty}(G)$ is $\mathcal{L}(G)$, and our result entails that a locally compact group $G$ is amenable if and only if $\mathcal{L}(G)$ is 1 -injective as an operator $A(G)$-module. We dedicate an entire section to applications of our general result, where we prove that closed quantum subgroups (in the sense of Vaes) of amenable quantum groups are amenable, and provide a decomposition result in the spirit of Haagerup [42] for completely bounded $L^{1}(\widehat{\mathbb{G}})$ module maps on $L^{\infty}(\widehat{\mathbb{G}})$.

Arguably the biggest open problem in abstract harmonic analysis on locally compact quantum groups is the duality of amenability and co-amenability. In the group setting, this is Leptin's theorem [68], which states that a locally compact group $G$ is amenable if and only if its Fourier algebra $A(G)$ has a bounded approximate identity. In the quantum group setting, many partial results have been obtained over the years. In 1996, Ruan showed that a compact Kac algebra $\mathbb{G}$ is co-amenable if and only if its discrete dual $\widehat{\mathbb{G}}$ is amenable [90, Theorem 4.5]. Ten years later, Tomatsu generalized this equivalence to arbitrary compact quantum groups [104, Theorem 3.8]. Tomatsu's argument relies on the specific modular theory of discrete quantum groups in order to apply the Powers-Størmer inequality in a crucial step. As a final application of our main result, we give a considerably simplified proof of Tomatsu's theorem which avoids the use of modular theory and the Powers-Størmer inequality, suggesting that it may be amenable to generalization beyond the compact setting.

In chapter 5 we introduce a notion of inner amenability for locally compact quantum groups and study its connection to relative injectivity of $L^{\infty}(\widehat{\mathbb{G}})$ as an operator $L^{1}(\widehat{\mathbb{G}})$-module. We show that, in general, inner amenability of $\mathbb{G}$ implies relative 1-injectivity of $L^{\infty}(\widehat{\mathbb{G}})$, and the converse holds in the group setting. That is, a locally compact group $G$ is inner amenable if and only if $\mathcal{L}(G)$ is relatively 1-injective as an operator $A(G)$-module. Hence, 1-injectivity of $\mathcal{L}(G)$ is to relative 1-injecivity of $\mathcal{L}(G)$ what amenability is to inner amenability. 
In his memoir [52], Johnson showed that the group algebra $L^{1}(G)$ of a locally compact group $G$ is (operator) amenable if and only if $G$ is amenable. In the breakthrough paper [89], Ruan established the dual result, showing that the Fourier algebra $A(G)$ of a locally compact group $G$ is operator amenable precisely when $G$ is amenable. In the language of biflatness, one may interpret their combined results as the equivalence

$$
L^{1}(G) \text { is operator 1-biflat } \Leftrightarrow A(G) \text { is operator 1-biflat. }
$$

We generalize the above equivalence to arbitrary locally compact quantum groups.

In addition to the above, the thesis contains the solutions to the following open problems:

- Inclusion of completely bounded $\mathcal{T}\left(L^{2}(\mathbb{G})\right)$-module maps, posed by $\mathrm{Hu}$, Neufang and Ruan [49, Remark 7.4(ii)].

- Equivalence of amenability and topological amenability, posed by Bédos and Tuset $[6, \S 3]$.

- $G$-amenability of $\mathcal{L}(G)$, posed by Lau and Paterson [67, Example 5].

Moreover, we partially resolve the following open problems:

- Relative operator projectivity of $A(G)$, posed by Forrest, Lee and Samei $[33, \S 4]$.

- Relative operator biflatness of $A(G)$, posed by Runde [92, Problem 33].

The ideas and results in this thesis suggest several new avenues of future research which we summarize in the final section. 


\section{Chapter 2}

\section{Preliminaries}

In 1925, our view of the quantum world drastically changed with the advent of Heisenberg's matrix mechanics. He showed that we may accurately describe quantum phenomena by interpreting time dependent variables as infinite matrices, or linear operators, rather than functions. This "quantization" of functions led to the development of operator algebras through the pioneering work of Murray and von Neumann, which in turn motivated mathematicians to quantize other areas of mathematics. One such quantization, mainly developed by Effros-Ruan and Blecher-Paulsen, is the theory of operator spaces, which may be viewed as a quantization of Banach space theory. Another important example is the quantization of abstract harmonic analysis. This procedure, culminating in theory of locally compact quantum groups, took over 40 years to be completed and includes the work of several prominent mathematicians such as Enock, Eymard, Kac, Krein, Kustermans, Schwartz, Takesaki, Tannaka, Vaes and Vainerman. The resulting theory lies at the confluence of Banach algebras and abstract harmonic analysis, operator algebras and operator spaces.

We therefore begin the thesis with a review of the necessary background from the latter fields, assuming the reader is familiar with general functional analysis. 


\section{$2.1 \quad$ Locally Compact Groups}

Definition 2.1.1. A topological group is a set $G$ that has the structure of a group and of a Hausdorff topological space such that the group operations $(s, t) \mapsto s t$ and $s \mapsto s^{-1}$ are continuous (where the topology on $G \times G$ is the product topology). If $G$ is locally compact, it is called a locally compact group.

Definition 2.1.2. Let $G$ be a topological group. A left (respectively, right) Haar measure on $G$ is a nonzero regular Borel measure $\mu$ that satisfies $\mu(s A)=\mu(A)$ (respectively, $\mu(A s)=\mu(A))$ for every Borel set $A \subseteq G$ and every $s \in G$.

Although there are important examples of non-locally compact groups - e.g., infinite-dimensional Banach spaces under addition, and unitary groups of infinitedimensional von Neumann algebras (cf. §2.4) - throughout the thesis we will almost solely be concerned with locally compact groups for the following reason.

Theorem 2.1.3. Every locally compact group possesses a left and a right Haar measure which are unique up to positive multiplicative constants.

Given a left Haar measure $\mu_{G}$ on a locally compact group $G$, for each $t \in G$ the formula $\mu_{t}(A)=\mu_{G}(A t)$ defines a regular Borel measure on $G$. By translational invariance of $\mu_{G}$, we have $\mu_{t}(s A)=\mu_{G}(s A t)=\mu_{G}(A t)=\mu_{t}(A)$ for every Borel subset $A$ and every $s \in G$. Then $\mu_{t}$ is a left Haar measure, so there exists a positive number $\Delta(t)$ such that $\mu_{t}=\Delta(t) \mu_{G}$. The function $\Delta: G \rightarrow \mathbb{R}$ defined in this way is called the modular function of $G$. It is easily seen that $\Delta$ is determined by the group and does not depend on a particular Haar measure.

Let $L^{\infty}(G)$ be the space of essentially bounded complex-valued Haar measurable functions on a locally compact group $G$. A bounded linear functional $m: L^{\infty}(G) \rightarrow \mathbb{C}$ is called a left invariant mean if

$$
\langle m, 1\rangle=\|m\|=1 \quad \text { and } \quad m(f \cdot s)=m(f)
$$


for all $s \in G$ and $f \in L^{\infty}(G)$, where $f \cdot s(t)=f(s t)$. A locally compact group $G$ is amenable if there is a left invariant mean on $L^{\infty}(G)$. By the Markov-Kakutani fixed point theorem, any abelian locally compact group is amenable. Compact groups are also amenable as the finite (left=right) Haar measure defines a left invariant mean via integration. The canonical example of a non-amenable group is $\mathbb{F}_{2}$, the free group on two generators. As closed subgroups of amenable groups are amenable (see [92, Theorem 1.2.7] for example), any locally compact group $G$ containing $\mathbb{F}_{2}$ as a closed subgroup is not amenable. For example, the general linear group $G L(n, \mathbb{R})$ of invertible $n \times n$ matrices and the special linear group $S L(n, \mathbb{R})=\{s \in G L(n, \mathbb{R}) \mid$ $\operatorname{det}(s)=1\}$ are not amenable for $n \geq 2$.

Definition 2.1.4. Let $G$ be a locally compact abelian group and let $\mathbb{T}$ denote the group of complex numbers of modulus one. A character on $G$ is a continuous homomorphism $\chi: G \rightarrow \mathbb{T}$.

The set of characters on a locally compact abelian group $G$, denoted $\widehat{G}$, forms an abelian group under pointwise multiplication, and is called the dual group of $G$. Given the topology of compact convergence, the dual group becomes a locally compact abelian group such that each $s \in G$ induces a character $\Phi(s)$ on $\widehat{G}$ via $\Phi(s)(\chi)=\chi(s)$. In fact, these are all the characters.

Theorem 2.1.5 (Pontrjagin Duality Theorem). Let $G$ be a locally compact abelian group. The mapping $G \ni s \mapsto \Phi(s) \in \widehat{\widehat{G}}$ is a homeomorphism of topological groups.

If $X$ is a locally compact Hausdorff space, we denote by $C_{0}(X)$ the space of continuous functions on $X$ vanishing at infinity. We also denote by $L^{1}(G)$, the Lebesgue space of integrable functions with respect to a fixed left Haar measure $d s:=d \mu_{G}(s)$ on a locally compact group $G$. When $G$ is abelian, the Fourier transform is the mapping $\mathcal{F}: L^{1}(G) \rightarrow C_{0}(\widehat{G})$ given by

$$
\mathcal{F}(f)(\chi)=\int_{G} \overline{\chi(s)} f(s) d s, \quad f \in L^{1}(G), \chi \in \widehat{G} .
$$




\subsection{Banach Algebras and Banach Modules}

Definition 2.2.1. A Banach algebra is an algebra $\mathcal{A}$ over the field of complex numbers equipped with a norm with respect to which it is a Banach space and which satisfies $\|a b\| \leq\|a\|\|b\|$ for all $a, b \in \mathcal{A}$. The Banach algebra $\mathcal{A}$ is said to

- be unital if it contains a multiplicative identity, which we denote by $e$;

- have a bounded right approximate identity if there exists a bounded net $\left(e_{\alpha}\right)_{\alpha \in A}$ in $\mathcal{A}$ satisfying

$$
\lim _{\alpha} a e_{\alpha}=a, \quad a \in \mathcal{A}
$$

There are analogous notions of bounded left (respectively, two-sided) approximate identities.

Remark 2.2.2. In what follows, if $\mathcal{A}$ is a unital Banach algebra, a unital subalgebra of $\mathcal{A}$ will mean a subalgebra $\mathcal{B} \subseteq \mathcal{A}$ containing the identity of $\mathcal{A}$.

Given a Banach algebra $\mathcal{A}$, its unitization is the unital Banach algebra $\mathcal{A}^{+}:=$ $\mathcal{A} \oplus_{1} \mathbb{C}$ with norm and multiplication given by

$\|(a, \alpha)\|=\|a\|+|\alpha| \quad$ and $\quad(a, \alpha)(b, \beta)=(a b+\beta a+\alpha b, \alpha \beta), \quad a, b \in \mathcal{A}, \alpha, \beta \in \mathbb{C}$.

Definition 2.2.3. Let $\mathcal{A}$ be a Banach algebra. A Banach space $X$ is a right Banach $\mathcal{A}$-module if it is a right $\mathcal{A}$-module for which $\|x \cdot a\| \leq\|x\|\|a\|$, for all $a \in \mathcal{A}$ and $x \in X$. In other words, the multiplication map $m_{X}: X \otimes^{\gamma} \mathcal{A} \rightarrow X$ is contractive for the Banach space projective tensor product $\otimes^{\gamma}$. We denote by $\langle X \cdot \mathcal{A}\rangle$ the closed linear span of $X \cdot \mathcal{A}:=\{x \cdot a \mid x \in X, a \in \mathcal{A}\}$. Left Banach $\mathcal{A}$-modules and Banach $\mathcal{A}$-bimodules are defined analogously.

When $\mathcal{A}$ has a bounded right approximate identity, then Cohen's factorization theorem asserts the following: for any right Banach $\mathcal{A}$-module $X$, and any $x \in\langle X \cdot \mathcal{A}\rangle$, 
there exists an $a \in \mathcal{A}$ and $y \in\langle X \cdot \mathcal{A}\rangle$ such that $x=y \cdot a$. Similar statements hold for left Banach $\mathcal{A}$-modules and Banach $\mathcal{A}$-bimodules.

Given a Banach algebra $\mathcal{A}$, there is a canonical $\mathcal{A}$-bimodule structure on $\mathcal{A}^{*}$ given by

$$
\langle a \cdot \varphi, b\rangle=\langle\varphi, b a\rangle \quad \text { and } \quad\langle\varphi \cdot a, b\rangle=\langle\varphi, a b\rangle, \quad \varphi \in \mathcal{A}^{*}, a, b \in \mathcal{A} .
$$

There are also left and right Arens products on the bidual $\mathcal{A}^{* *}$, denoted $\square$ and $\diamond$, respectively, defined by

$$
\langle m \square n, \varphi\rangle=\langle m, n \square \varphi\rangle \quad \text { and } \quad\langle m \diamond n, \varphi\rangle=\langle n, \varphi \diamond m\rangle \quad m, n \in \mathcal{A}^{* *}, \varphi \in \mathcal{A}^{*},
$$

where $n \square \varphi$ and $\varphi \diamond m$ are the elements in $\mathcal{A}^{*}$ given by

$$
\langle n \square \varphi, a\rangle=\langle n, \varphi \cdot a\rangle \quad \text { and } \quad\langle\varphi \diamond m, a\rangle=\langle m, a \cdot \varphi\rangle, \quad a \in \mathcal{A} .
$$

It follows that $\left(\mathcal{A}^{* *}, \square\right)$ and $\left(\mathcal{A}^{* *}, \diamond\right)$ are Banach algebras extending the product in $\mathcal{A}$. When the left and right Arens products on $\mathcal{A}^{* *}$ coincide, $\mathcal{A}$ is said to be Arens regular. For example, any $C^{*}$-algebra (see below) is Arens regular.

Definition 2.2.4. An involution on an algebra $\mathcal{A}$ is a map ${ }^{*}: \mathcal{A} \rightarrow \mathcal{A}$ that satisfies

$$
(a+b)^{*}=a^{*}+b^{*},(\lambda a)^{*}=\bar{\lambda} a^{*},(a b)^{*}=b^{*} a^{*}, a^{* *}=a
$$

for all $a, b \in \mathcal{A}, \lambda \in \mathbb{C}$. An algebra equipped with an involution is called an involutive algebra.

- An involutive Banach algebra whose *-operation is an isometry is called a $B a$ nach *algebra.

- An involutive Banach algebra $\mathcal{A}$ that satisfies

$$
\left\|a^{*} a\right\|=\|a\|^{2}, \quad a \in \mathcal{A},
$$


is called a $C^{*}$-algebra.

The prototypical example of a $C^{*}$-algebra is $\mathcal{B}(H)$, the space of bounded linear operators on a Hilbert space, with the operator norm and involution given by the adjoint operation. The space $\mathcal{B}(H)$ is the dual of the Banach space of trace-class operators, denoted $\mathcal{T}(H)$, where an element $\rho \in \mathcal{B}(H)$ is called trace-class if $\operatorname{tr}|\rho|<$ $\infty$, with tr denoting the canonical trace of an operator with respect to an orthonormal basis of $H$. The duality between $\mathcal{B}(H)$ and $\mathcal{T}(H)$ is given by

$$
\langle T, \rho\rangle=\operatorname{tr}(\rho T), \quad \rho \in \mathcal{T}(H), T \in \mathcal{B}(H) .
$$

Given vectors $\xi, \eta \in H$, we denote the corresponding rank-1 operator $\beta \mapsto\langle\beta, \eta\rangle \xi$, $\beta \in H$, by $|\xi\rangle\langle\eta|$. The closed linear span of all rank-1 operators in $\mathcal{B}(H)$ forms the $C^{*}$-algebra subalgebra $\mathcal{K}(H)$ of compact operators on $H$. As a Banach space, it is the predual of $\mathcal{T}(H)$, where the duality is given as above by

$$
\langle\rho, y\rangle=\operatorname{tr}(\rho y), \quad \rho \in \mathcal{T}(H), y \in \mathcal{K}(H) .
$$

We remark that the dualities $\mathcal{K}(H)=\mathcal{T}(H)^{*}$ and $\mathcal{T}(H)^{*}=\mathcal{B}(H)$ are non-commutative analogues of the well-known dualities $c_{0}^{*}=\ell^{1}$ and $\left(\ell^{1}\right)^{*}=\ell^{\infty}$.

Much of abstract harmonic analysis concerns the study of Banach algebras associated to a locally compact group. Here we discuss the main examples that will be important throughout the thesis, with $G$ denoting a locally compact group with fixed left Haar measure $d t$.

The space $C_{0}(G)$ with the uniform norm, pointwise multiplication and involution given by complex conjugation, forms a commutative $C^{*}$-algebra. In fact, every commutative $C^{*}$-algebra is of the form $C_{0}(X)$ for a locally compact Hausdorff space $X$ [100, Theorem 4.4], suggesting that $C^{*}$-algebras play the role of "non-commutative topological spaces".

By the Riesz representation theorem, the Banach space dual $C_{0}(G)^{*}$ is isometri- 
cally isomorphic to $M(G)$, the space of finite complex regular Borel measures on $G$ with total variation norm. The duality is given by

$$
\langle\mu, f\rangle=\int_{G} f(s) d \mu(s), \quad \mu \in M(G), f \in C_{0}(G) .
$$

The space $M(G)$ becomes a Banach algebra when equipped with the convolution product, defined by

$$
\langle\mu * \nu, f\rangle=\int_{G} \int_{G} f(s t) d \mu(s) d \nu(t), \quad \mu, \nu \in M(G), f \in C_{0}(G) .
$$

The resulting algebra is called the measure algebra of $G$, and is commutative if and only if $G$ is abelian. With the involution $\mu^{*}(A)=\overline{\mu\left(A^{-1}\right)}, A \subseteq G$ Borel, $M(G)$ becomes a Banach *-algebra.

We may identify $L^{1}(G)$ with a norm closed ideal in $M(G)$ consisting of those measures that are absolutely continuous with respect to the Haar measure. With this identification, the convolution of $f$ and $g$ in $L^{1}(G)$ is the function

$$
f * g(s)=\int_{G} f(t) g\left(t^{-1} s\right) d t, \quad s \in G .
$$

The restricted involution on $L^{1}(G)$ takes the form $f^{o}(s)=\Delta\left(s^{-1}\right) \overline{f\left(s^{-1}\right)}, s \in G$. Under the convolution product and the above involution, $L^{1}(G)$ also becomes a Banach *-algebra, called the group algebra of $G$. The associated bimodule actions on its dual $L^{\infty}(G)$ are given by

$$
f * h(s)=\int_{G} h(s t) f(t) d t \quad \text { and } \quad h * f(s)=\int_{G} h(t s) f(t) d t
$$

where $f \in L^{1}(G)$ and $h \in L^{\infty}(G)$. Let $C_{b}(G)$ denote the space of bounded continuous functions on $G$. Given any such $h \in L^{\infty}(G)$ and $f \in L^{1}(G)$, it turns out that $g:=h * f \in C_{b}(G)$ is always left uniformly continuous, meaning that the map $G \ni s \mapsto g \cdot s \in C_{b}(G)$ is continuous with respect to the supremum norm. The space 
of such functions is denoted $\operatorname{LUC}(G)$ and is a $C^{*}$-subalgebra $C_{b}(G)$. Moreover, we have the following equalities:

$$
\operatorname{LUC}(G)=\left\langle L^{\infty}(G) * L^{1}(G)\right\rangle=L^{\infty}(G) * L^{1}(G),
$$

where the last equality follows from Cohen's factorization theorem as $L^{1}(G)$ always has a bounded approximate identity (see [32, Proposition 2.42], for instance). Analogously, the space of right uniformly continuous functions is denoted $\operatorname{RUC}(G)$ and satisfies

$$
\operatorname{RUC}(G)=\left\langle L^{1}(G) * L^{\infty}(G)\right\rangle=L^{1}(G) * L^{\infty}(G) .
$$

Definition 2.2.5. An element $a$ in a Banach ${ }^{*}$-algebra $\mathcal{A}$ said to be positive, denoted $a \geq 0$, if $a=b^{*} b$ for some $b \in \mathcal{A}$. We denote the set of positive elements by $\mathcal{A}_{\geq 0}$.

A linear map $\varphi: \mathcal{A} \rightarrow \mathcal{B}$ between Banach *-algebras is said to be positive if $\varphi\left(\mathcal{A}_{\geq 0}\right) \subseteq \mathcal{B}_{\geq 0}$. In the special case where $\mathcal{B}=\mathbb{C}$, we say that $\varphi$ is a positive linear functional. The collection of positive linear functionals of norm one, denoted $\mathcal{S}(\mathcal{A})$, is called the state space of $\mathcal{A}$. When $\mathcal{A}$ is a $C^{*}$-algebra, $\mathcal{S}(\mathcal{A})$ forms a convex subset of the closed unit ball of $\mathcal{A}^{*}[86$, Proposition 2.3.7]. Its extreme points are called pure states. We say that a state $\varphi \in \mathcal{S}(\mathcal{A})$ is tracial if $\varphi(a b)=\varphi(b a)$ for all $a, b \in \mathcal{A}$, and faithful if $\varphi(a)=0$ implies $a=0$ for all $a \in \mathcal{A}_{\geq 0}$.

Remark 2.2.6. If $\mathcal{A}$ is a unital Banach *-algebra, then a positive linear functional $\varphi$ is a state if and only if $\varphi(1)=1[100$, Lemma 9.9]. In this case, $\mathcal{S}(\mathcal{A})$ is a weak* closed subset of the closed unit ball of $\mathcal{A}^{*}$, and is therefore compact by Alaoglu's theorem.

Positive linear functionals on $C^{*}$-algebras are necessarily bounded, and similar to the Jordan decomposition of signed measures on locally compact spaces, any bounded linear functional on a $C^{*}$-algebra can be uniquely decomposed into a sum of four positive linear functionals [100]. 
Definition 2.2.7. A ${ }^{*}$-representation of a Banach ${ }^{*}$-algebra $\mathcal{A}$ is a ${ }^{*}$-homomorphism $\pi: \mathcal{A} \rightarrow \mathcal{B}\left(H_{\pi}\right)$, where $H_{\pi}$ is a Hilbert space. We say that $\pi$ is:

- spatially nondegenerate, or s.nd. for short, if $\pi(\mathcal{A}) H_{\pi}:=\{\pi(a) \xi: a \in \mathcal{A}, \quad \xi \in$ $\left.H_{\pi}\right\}$ is dense in $H_{\pi}$;

- *-representation!cyclic if there is a vector $\xi \in H_{\pi}$ such that $\pi(\mathcal{A}) \xi$ is dense in $H_{\pi}$

- irreducible if there are no non-trivial proper invariant subspaces of $H_{\pi}$ under $\pi(\mathcal{A})$

- faithful if $\pi$ is injective.

Two *-representations $\pi_{1}$ and $\pi_{2}$ are said to be unitarily equivalent if there exists a unitary $U: H_{\pi_{1}} \rightarrow H_{\pi_{2}}$ such that $\pi_{2}(a)=U \pi_{1}(a) U^{*}$ for all $a \in \mathcal{A}$.

Any *-representation of a $C^{*}$-algebra is bounded [86, Proposition 2.3.17].

For $f \in L^{1}(G)$, define

$$
\|f\|_{*}=\sup \left\{\|\pi(f)\| \mid \pi: L^{1}(G) \rightarrow \mathcal{B}\left(H_{\pi}\right) \quad \text { is a s.nd. *-representation }\right\} .
$$

It is easily seen that $\|\cdot\|_{*}$ is a norm on $L^{1}(G)$ satisfying $\|\cdot\|_{*} \leq\|\cdot\|_{1}$, whose completion becomes a $C^{*}$-algebra, denoted $C^{*}(G)$, called the universal group $C^{*}$-algebra of $G$.

Given a ${ }^{*}$-representation $\pi: \mathcal{A} \rightarrow \mathcal{B}(H)$ of a Banach *-algebra and a vector $\xi \in H_{\pi}$, the function $\varphi(x)=\langle\pi(x) \xi, \xi\rangle, x \in \mathcal{A}$, defines a positive linear functional on $\mathcal{A}$. The converse of this result is known as the GNS construction.

Theorem 2.2.8. If $\varphi$ is a positive linear functional on a Banach *-algebra $\mathcal{A}$, and $\mathcal{A}$ has a bounded approximate identity, there is a unique cyclic ${ }^{*}$-representation $(\pi, \xi)$ of $\mathcal{A}$ on a Hilbert space $H_{\varphi}$ (up to unitary equivalence) such that $\varphi(x)=\langle\pi(x) \xi, \xi\rangle$ for all $x \in \mathcal{A}$. Moreover, if $\varphi \in \mathcal{S}(\mathcal{A})$, then $\varphi$ is pure if and only if $\pi$ is irreducible. 
Given two $C^{*}$-algebras $\mathcal{A}$ and $\mathcal{B}$, acting on Hilbert spaces $H$ and $K$, respectively, the algebraic tensor product $\mathcal{A} \otimes \mathcal{B}$ acts naturally on the Hilbert space tensor product $H \otimes_{2} K$, and the norm closure of $\mathcal{A} \otimes \mathcal{B}$ inside $\mathcal{B}\left(H \otimes_{2} K\right)$ is a $C^{*}$-algebra called the minimal $C^{*}$-tensor product of $\mathcal{A}$ and $\mathcal{B}$, denoted $\mathcal{A} \otimes_{\min } \mathcal{B}$. It can be shown that this definition is independent of the Hilbert space representation [86, §3.2]. Furthermore, denoting the identity map by id, if $\varphi \in \mathcal{A}^{*}$, then the right slice map $\varphi \otimes$ id $: \mathcal{A} \otimes \mathcal{B} \rightarrow \mathcal{B}$ defined on elementary tensors by

$$
(\varphi \otimes \mathrm{id})(a \otimes b)=\varphi(a) b, \quad a \in \mathcal{A}, b \in \mathcal{B},
$$

extends uniquely to a bounded linear map (still denoted) $\varphi \otimes$ id $: \mathcal{A} \otimes_{\min } \mathcal{B} \rightarrow \mathcal{B}[86$, $\S 3.2]$.

\section{$2.3 \quad$ von Neumann Algebras}

Definition 2.3.1. A von Neumann algebra $M$ acting on a Hilbert space $H$ is a unital $C^{*}$-subalgebra of $\mathcal{B}(H)$ that is closed under the strong operator topology. The unit in a von Neumann algebra $M$ will be denoted by 1 .

Let $(X, \Sigma, \mu)$ be a localizable measure space (e.g., a locally compact group). Under pointwise multiplication, the space of (complex-valued) essentially bounded $\mu$ measurable functions $L^{\infty}(X, \mu)$ becomes a commutative von Neumann algebra when viewed as the algebra of multiplication operators on $L^{2}(X, \mu)$. In fact, every commutative von Neumann algebra arises in this fashion for a localizable measure space $(X, \Sigma, \mu)[86$, Theorem 5.3.4], suggesting that von Neumann algebras play the role of "non-commutative measure spaces".

For a subset $\mathcal{S}$ of $B(H)$, the commutant of $\mathcal{S}$, denoted $\mathcal{S}^{\prime}$, is the set of operators in $\mathcal{B}(H)$ that commute with every element of $\mathcal{S}$, that is

$$
\mathcal{S}^{\prime}=\{T \in \mathcal{B}(H): T S=S T \text { for all } S \in \mathcal{S}\}
$$


The double commutant or bicommutant of $\mathcal{S}$ is defined by $\mathcal{S}^{\prime \prime}=\left(\mathcal{S}^{\prime}\right)^{\prime}$. von Neumann's celebrated bicommutant theorem relates this purely algebraic concept with the topological structure of von Neumann algebras.

Theorem 2.3.2. If $M$ is a self-adjoint unital subalgebra of $\mathcal{B}(H)$, then the weak*, weak and strong operator closures of $M$ coincide with $M^{\prime \prime}$.

A von Neumann algebra $M \subseteq \mathcal{B}(H)$ is said to be a factor if $\mathcal{Z}(M):=M \cap M^{\prime}=$ $\mathbb{C} 1$. Factors are the basic building blocks of von Neumann algebras [100, §IV.8]. We say that $M$ is a factor of type $I I_{1}$ if it has a unique normal faithful tracial state.

A distinguishing feature of a von Neumann algebra $M \subseteq \mathcal{B}(H)$ amongst $C^{*}$ algebras is that it is isometrically isomorphic to the dual of a unique Banach space $M_{*}$, called the predual of $M$. As the predual $M_{*}$ sits isometrically in $M^{*}$, it inherits a canonical Banach $M$-bimodule structure via

$$
\langle x \cdot \omega, y\rangle=\langle\omega, y x\rangle \quad \text { and } \quad\langle\omega \cdot x, y\rangle=\langle\omega, x y\rangle, \quad \omega \in M_{*}, x, y \in M .
$$

Definition 2.3.3. If $M$ and $N$ are von Neumann algebras, then a linear map $\Phi$ : $M \rightarrow N$ is said to be normal if it is weak*-weak* continuous.

If $M \subseteq \mathcal{B}(H)$, observe that for $\xi, \eta \in H$, the linear functional $\omega_{\xi, \eta}: M \rightarrow \mathbb{C}$ defined by $\left\langle\omega_{\xi, \eta}, x\right\rangle=\langle x \xi, \eta\rangle, x \in M$, is normal. For simplicity, we denote $\omega_{\xi, \xi}$ by $\omega_{\xi}$.

If $G$ is a locally compact group, then $L^{\infty}(G)$ is a von Neumann algebra with predual $L^{1}(G)[32, \S 2.3]$. For the remainder of the thesis, elements of $L^{\infty}(G)$ will be viewed as functions and/or multiplication operators when convenient. We will denote functions by $f$ and their corresponding multiplication operators by $M_{f}$.

Many important von Neumann algebras in abstract harmonic analysis arise from strongly continuous unitary representations of a locally compact group $G$, the two main examples being the left and right regular representations, given, respectively, by 
$\lambda: G \rightarrow \mathcal{B}\left(L^{2}(G)\right)$ and $\rho: G \rightarrow \mathcal{B}\left(L^{2}(G)\right)$, where

$$
\lambda(s) \xi(t)=\xi\left(s^{-1} t\right) \quad \text { and } \quad \rho(s) \xi(t)=\Delta(s)^{1 / 2} \xi(t s)
$$

for $\xi \in L^{2}(G), s, t \in G$. It follows that

$$
\mathcal{L}(G):=\overline{\operatorname{span}\{\lambda(s): s \in G\}}^{S O T} \quad \text { and } \quad \mathcal{R}(G):=\overline{\operatorname{span}\{\rho(s): s \in G\}}^{S O T}
$$

are von Neumann algebras in $\mathcal{B}\left(L^{2}(G)\right)$, called the left and right group von Neumann algebras, respectively. Furthermore, they satisfy the following commutation relations:

$$
\mathcal{L}(G)^{\prime}=\mathcal{R}(G) \quad \text { and } \quad \mathcal{R}(G)^{\prime}=\mathcal{L}(G)
$$

Remark 2.3.4. By lifting the left regular representation to $\lambda: L^{1}(G) \rightarrow \mathcal{B}\left(L^{2}(G)\right)$ via:

$$
\langle\lambda(f), \omega\rangle=\int_{G} f(s)\langle\lambda(s), \omega\rangle d s, \quad f \in L^{1}(G), \omega \in \mathcal{T}\left(L^{2}(G)\right),
$$

it follows that

$$
\mathcal{L}(G)={\overline{\operatorname{span}\left\{\lambda(f): f \in L^{1}(G)\right\}}}^{S O T} .
$$

If instead we take the norm closure in equation $(2.2)$, then we obtain a $C^{*}$-algebra, denoted $C_{\lambda}^{*}(G)$, known as the left reduced $C^{*}$-algebra. Similarly, we have the corresponding result for the right regular representation.

The set of coefficient functions of the left regular representation,

$$
A(G)=\left\{\psi: G \rightarrow \mathbb{C}: \psi(s)=\langle\lambda(s) \xi, \eta\rangle, \quad \xi, \eta \in L^{2}(G), \quad s \in G\right\}
$$

is called the Fourier algebra of $G$. It was shown by Eymard that, endowed with the norm

$$
\|\psi\|=\inf \{\|\xi\|\|\eta\|: \psi(\cdot)=\langle\lambda(\cdot) \xi, \eta\rangle\}
$$


$A(G)$ is a Banach algebra under pointwise multiplication [31, Proposition 3.4]. Furthermore, it is the predual of the left group von Neumann algebra $\mathcal{L}(G)$, where the duality is given by

$$
\langle\psi, \lambda(s)\rangle=\psi(s), \quad \psi \in A(G), s \in G .
$$

Eymard also showed that the space of functions $\varphi: G \rightarrow \mathbb{C}$ for which there exists a strongly continuous unitary representation $\pi: G \rightarrow \mathcal{B}\left(H_{\pi}\right)$ and $\xi, \eta \in H_{\pi}$ such that $\varphi(s)=\langle\pi(s) \xi, \eta\rangle, s \in G$, is a unital Banach algebra (with pointwise multiplication) under the norm

$$
\|\varphi\|=\sup _{f \in L^{1}(G),\|f\|_{*} \leq 1}\left|\int_{G} f(s) \varphi(s) d s\right|
$$

called the Fourier-Stieltjes algebra of $G$ [31, Proposition 2.16], denoted by $B(G)$. This algebra coincides with the Banach space dual of the group $C^{*}$-algebra $C^{*}(G)$.

When $G$ is abelian with dual group $\widehat{G}$, the algebra $A(\widehat{G})$ is simply the image of the Fourier transform $\mathcal{F}: L^{1}(G) \rightarrow C_{0}(\widehat{G})$. The dual mapping $\mathcal{F}^{*}: \mathcal{L}(\widehat{G}) \rightarrow L^{\infty}(G)$ therefore provides a von Neumann algebraic isomorphism. In this sense, $L^{\infty}(G)$ and $\mathcal{L}(G)$ are "dual" to one another, and it was precisely this correspondence that set the stage for the quantization of abstract harmonic analysis to non-abelian groups; a theory which utilizes non-commutative integration in an essential way. For details we refer the reader to [101].

Definition 2.3.5. A weight on a von Neumann algebra $M$ is a function $\varphi: M_{\geq 0} \rightarrow$ $[0, \infty]$ satisfying:

1. $\varphi(x+y)=\varphi(x)+\varphi(y)$ for all $x, y \in M_{\geq 0}$;

2. $\varphi(r x)=r \varphi(x)$ for all $r \in \mathbb{R}_{\geq 0}, \quad x \in M_{\geq 0}$.

The fact that weights may be viewed as non-commutative integrals is justified by the following canonical example. 
Example 2.3.6. If $(X, \Sigma, \mu)$ is a $\sigma$-finite measure space, then the function

$$
\varphi(f)=\int_{X} f(s) d \mu(s), \quad 0 \leq f \in L^{\infty}(X, \mu),
$$

is a weight on the commutative von Neumann algebra $L^{\infty}(X, \mu)$.

Given a weight $\varphi$ on a von Neumann algebra $M$, we adopt the following standard notations:

- $\mathcal{M}_{\varphi}^{+}=\left\{x \in M_{\geq 0}: \varphi(x)<\infty\right\}$

- $\mathcal{N}_{\varphi}=\left\{x \in M: \varphi\left(x^{*} x\right)<\infty\right\}$

- $\mathcal{M}_{\varphi}=\operatorname{span}\left\{x^{*} y: x, y \in \mathcal{N}_{\varphi}\right\}$

It follows that $\mathcal{N}_{\varphi}$ is a left ideal in $M$, and that $\mathcal{M}_{\varphi}=\operatorname{span} \mathcal{M}_{\varphi}^{+}$is a ${ }^{*}$-subalgebra of $M$ [101, Lemma VII.1.2]. We may therefore extend $\varphi$ linearly to $\mathcal{M}_{\varphi}$, which will also be denoted by $\varphi$.

In the classical setting of Example 2.3.6, $\mathcal{M}_{\varphi}$ and $\mathcal{N}_{\varphi}$ correspond to $L^{\infty}(X, \mu) \cap$ $L^{1}(X, \mu)$ and $L^{\infty}(X, \mu) \cap L^{2}(X, \mu)$, respectively. In general, we say that the weight $\varphi$ is:

- semi-finite if $\mathcal{M}_{\varphi}$ is weak ${ }^{*}$ dense in $M$;

- faithful if $\varphi(x)=0$ implies $x=0$ for all $x \in M_{\geq 0}$;

- normal if $\varphi\left(\sup _{\alpha} x_{\alpha}\right)=\sup _{\alpha} \varphi\left(x_{\alpha}\right)$ for every bounded increasing net $\left(x_{\alpha}\right)_{\alpha \in A}$ in $M_{\geq 0}$.

If $\varphi$ is a normal semi-finite faithful (n.s.f.) weight on a von Neumann algebra $M$, we may define an inner product on $\mathcal{N}_{\varphi}$ by

$$
\langle x, y\rangle=\varphi\left(y^{*} x\right), \quad x, y \in \mathcal{N}_{\varphi} .
$$


Denoting by $H_{\varphi}$ the Hilbert space completion of $\mathcal{N}_{\varphi}$, we obtain the canonical embed$\operatorname{ding} \Lambda_{\varphi}: \mathcal{N}_{\varphi} \rightarrow H_{\varphi}$ and a ${ }^{*}$-representation $\pi_{\varphi}: M \rightarrow B\left(H_{\varphi}\right)$ of $M$ given by

$$
\pi_{\varphi}(x)\left(\Lambda_{\varphi}(a)\right)=\Lambda_{\varphi}(x a), \quad x \in M, a \in \mathcal{N}_{\varphi} .
$$

The triple $\left(H_{\varphi}, \pi_{\varphi}, \Lambda_{\varphi}\right)$ is called the GNS-construction for $\varphi$, and we may identity $M \cong \pi_{\varphi}(M)$ as von Neumann algebras. At the level of the Hilbert space $H_{\varphi}$, the adjoint operation in $M$ generates a closable operator $\Lambda_{\varphi}(x) \mapsto \Lambda_{\varphi}\left(x^{*}\right), x \in \mathcal{N}_{\varphi}$, whose closure $S_{\varphi}$ has polar decomposition $J_{\varphi} \Delta_{\varphi}$, where $J_{\varphi}: H_{\varphi} \rightarrow H_{\varphi}$ is a conjugate linear isometry and $\Delta_{\varphi}$ is the modular operator. It follows that $\Delta_{\varphi}^{i t} M \Delta_{\varphi}^{-i t}=M$ for all $t \in \mathbb{R}$, which gives rise to the modular automorphism group $\left(\sigma_{t}^{\varphi}\right)_{t \in \mathbb{R}}$ of $\varphi$, defined by $\sigma_{t}^{\varphi}(x)=\Delta_{\varphi}^{i t} x \Delta_{\varphi}^{-i t}$, for $x \in M$.

An element $x \in M$ is said to be analytic with respect to $\varphi$ if the function $\mathbb{R} \ni$ $t \mapsto \sigma_{t}^{\varphi}(x) \in M$ has an extension to an analytic function $\mathbb{C} \ni z \mapsto \sigma_{z}^{\varphi}(x) \in M$, that is, for every $\omega \in M_{*}$, the function $\mathbb{C} \ni z \mapsto\left\langle\sigma_{z}^{\varphi}(x), \omega\right\rangle \in \mathbb{C}$ is analytic. We denote by $\mathcal{T}_{\varphi}$ the set of analytic elements. When $\varphi$ is tracial, that is, $\varphi\left(x^{*} x\right)=\varphi\left(x x^{*}\right), x \in \mathcal{N}_{\varphi}$, it follows that $\sigma_{t}^{\varphi}=\mathrm{id}_{M}$ for all $t \in \mathbb{R}$, the operator $S_{\varphi}=J_{\varphi}$ is an isometry, and the modular operator $\Delta_{\varphi}=1$.

In general, the conjugate linear isometry $J_{\varphi}$ obeys the formula

$$
J_{\varphi} \Lambda_{\varphi}(x)=\Lambda_{\varphi}\left(\sigma_{i / 2}^{\varphi}(x)^{*}\right), \quad x \in \mathcal{N}_{\varphi} \cap \mathcal{D}\left(\sigma_{i / 2}^{\varphi}\right),
$$

where $\mathcal{D}\left(\sigma_{i / 2}^{\varphi}\right)$ denotes the domain of $\sigma_{i / 2}^{\varphi}$. Letting $\mathcal{P}_{\varphi}=\overline{\left\{\Lambda_{\varphi}(x) J_{\varphi} \Lambda_{\varphi}(x) \mid x \in \mathcal{N}_{\varphi}\right\}} \subseteq$ $H_{\varphi}$, it follows that $\mathcal{P}_{\varphi}$ is a self-dual cone and that the quadruple $\left(\pi_{\varphi}(M), H_{\varphi}, J_{\varphi}, \mathcal{P}_{\varphi}\right)$ has the following properties:

1. $J_{\varphi} \pi_{\varphi}(M) J_{\varphi}=\pi_{\varphi}(M)^{\prime}$

2. $J_{\varphi} z J_{\varphi}=z^{*}$ for all $z \in \mathcal{Z}\left(\pi_{\varphi}(M)\right)$;

3. $J_{\varphi} \xi=\xi$ for all $\xi \in \mathcal{P}_{\varphi}$; 
4. $x J_{\varphi} x J_{\varphi}\left(\mathcal{P}_{\varphi}\right) \subseteq \mathcal{P}_{\varphi}$ for all $x \in \pi_{\varphi}(M)$.

In general, a quadruple $(N, H, J, \mathcal{P})$ consisting of a von Neumann algebra $N$ on a Hilbert space $H$, a conjugate linear isometry $J: H \rightarrow H$, and a self-dual cone $\mathcal{P}$ in $H$ is called a standard form if the above conditions (1) - (4) are satisfied. In this case, we simply say that $N$ is standardly represented on $H$. An important property of standard representations is that every normal state $\omega \in N_{*}$ is the restriction of the vector state $\omega_{\xi}$ to $N$ for a unique vector $\xi \in \mathcal{P}$ [40, Lemma 2.10]. For details on standard representations of von Neumann algebras we refer the reader to [40].

\subsection{Operator Spaces}

If $X$ is a linear space, for $m, n \in \mathbb{N}$ we let $M_{m, n}(X)$ denote the space of $m \times n$ matrices with entries in $X$. If $m=n$, we simply denote $M_{m, n}(X)$ by $M_{n}(X)$.

A matrix norm on a linear space $X$ is a sequence of norms $\left(\|\cdot\|_{n}\right)_{n \in \mathbb{N}}$ where $\|\cdot\|_{n}$ is defined on $M_{n}(X)$ for every $n \in \mathbb{N}$. An abstract operator space is a linear space $X$ together with a matrix norm such that each $\|\cdot\|_{n}$ is complete and satisfies

- R1 $\|x \oplus y\|_{m+n}=\max \left\{\|x\|_{m},\|y\|_{n}\right\}$,

- R2 $\|\alpha x \beta\|_{n} \leq\|\alpha\|\|x\|_{m}\|\beta\|$,

for all $m, n \in \mathbb{N}, x \in M_{m}(X), y \in M_{n}(X), \alpha \in M_{n, m}(\mathbb{C}), \beta \in M_{m, n}(\mathbb{C})$. Such a matrix norm is called an operator space matrix norm.

Given abstract operator spaces $X, Y$ and a linear mapping $\Phi: X \rightarrow Y$, for each $n \in \mathbb{N}$ there is a corresponding linear map $\Phi_{n}: M_{n}(X) \rightarrow M_{n}(Y)$ given by

$$
\Phi_{n}(x)=\left[\Phi\left(x_{i j}\right)\right]
$$

for all $x=\left[x_{i j}\right] \in M_{n}(X)$, called the $n^{t h}$ amplification of $\Phi$.

Definition 2.4.1. Let $X$ and $Y$ be abstract operator spaces. A linear map $\Phi: X \rightarrow$ $Y$ is called: 
- completely bounded if $\|\Phi\|_{c b}:=\sup \left\{\left\|\Phi_{n}\right\|: n \in \mathbb{N}\right\}<\infty$;

- completely contractive if $\|\Phi\|_{c b} \leq 1$;

- a complete isometry if $\Phi_{n}$ is an isometry for all $n \in \mathbb{N}$;

- a complete quotient map if $\Phi_{n}$ is a quotient map for all $n \in \mathbb{N}$.

- an exact complete quotient map if $\Phi_{n}$ is an exact quotient map for all $n \in \mathbb{N}$.

The prototypical example of an abstract operator space is a closed subspace $X$ of $\mathcal{B}(H)$ for some Hilbert space $H$. The matrix norms are defined by the identification $M_{n}(X) \subseteq M_{n}(\mathcal{B}(H)) \cong \mathcal{B}\left(H^{n}\right)$, where $H^{n}$ is the $n$-fold Hilbert space direct sum and $n \in \mathbb{N}$. These are known as concrete operator spaces, and in fact, there is a bijective correspondence.

Theorem 2.4.2 (Ruan's Theorem). [87] Every abstract operator space is completely isometrically isomorphic to a concrete operator space.

In what follows, we will therefore omit the adjectives abstract and concrete and simply use the term operator space. Also, we denote by $M_{\infty}, K_{\infty}$ and $T_{\infty}$ the space of all bounded, compact and trace-class operators on a separable Hilbert space, respectively.

Let $H$ and $K$ be a Hilbert spaces and let $X$ and $Y$ be self-adjoint unital subspaces of $\mathcal{B}(H)$ and $\mathcal{B}(K)$, respectively. Then $M_{n}(X)$ and $M_{n}(Y)$ are self-adjoint subspaces of $M_{n}(\mathcal{B}(H))$ and $M_{n}(\mathcal{B}(K))$ for $n \geq 1$. A linear map $\Phi: X \rightarrow Y$ is completely positive if $\Phi_{n}$ is positive for all $n \in \mathbb{N}$, where positivity is taken with respect to the natural positive cones in $M_{n}(\mathcal{B}(H))$ and $M_{n}(\mathcal{B}(K))$, respectively. In this case, it follows that $\Phi$ is automatically completely bounded with $\|\Phi\|_{c b}=\|\Phi\|=\|\Phi(1)\|$ [80, Proposition 3.6]. We denote the set of completely positive maps from $X$ to $Y$ by $\mathcal{C P}(X, Y)$. If $X=\mathcal{A}$ and $Y=\mathcal{B}$ are $C^{*}$-algebras, then there is a convenient characterization of complete positivity [100, Corollary 3.4]. 
Proposition 2.4.3. Let $\mathcal{A}$ and $\mathcal{B}$ be $C^{*}$-algebras. Then a linear map $\Phi: \mathcal{A} \rightarrow \mathcal{B}$ is completely positive if and only if

$$
\sum_{i, j=1}^{n} b_{i}^{*} \Phi\left(a_{i}^{*} a_{j}\right) b_{j} \geq 0
$$

for all $a_{1}, \ldots, a_{n} \in \mathcal{A}, b_{1}, \ldots, b_{n} \in \mathcal{B}$ and $n \in \mathbb{N}$.

Another important fact that we will use is that a unital complete contraction $\Phi: \mathcal{A} \rightarrow \mathcal{B}$ between unital $C^{*}$-algebras is automatically completely positive [80, Proposition 2.12]. Moreover, in this case, the multiplicative domain of $\Phi$, defined as

$$
\mathcal{M D}_{\Phi}=\left\{a \in \mathcal{A} \mid \Phi\left(a^{*} a\right)=\Phi(a)^{*} \Phi(a) \quad \text { and } \quad \Phi\left(a a^{*}\right)=\Phi(a) \Phi(a)^{*}\right\},
$$

is a $C^{*}$-subalgebra of $\mathcal{A}$ such that $\Phi$ is an $\mathcal{M D}_{\Phi}$-bimodule map [12], that is, $\Phi\left(a_{1} a a_{2}\right)=$ $\Phi\left(a_{1}\right) \Phi(a) \Phi\left(a_{2}\right)$ for $a_{1}, a_{2} \in \mathcal{M D}_{\Phi}$ and $a \in \mathcal{A}$.

Given operator spaces $X$ and $Y$, we denote the set of completely bounded mappings from $X$ to $Y$ by $\mathcal{C B}(X, Y)$. Note that each $\Phi \in M_{n}(\mathcal{C B}(X, Y))$ may be identified with an element of $\mathcal{C B}\left(X, M_{n}(Y)\right)$, for $n \in \mathbb{N}$. By defining

$$
\|\Phi\|_{M_{n}(\mathcal{C B}(X, Y))}:=\|\Phi\|_{\mathcal{C B}\left(X, M_{n}(Y)\right)}
$$

it follows that $\|\cdot\|_{M_{n}(\mathcal{C B}(X, Y))}$ is an operator space matrix norm, turning $\mathcal{C B}(X, Y)$ into an operator space in its own right. As is customary, if $X=Y$ we simply write $\mathcal{C B}(X)$ for $\mathcal{C B}(X, X)$. When $M$ and $N$ are von Neumann algebras, the space of normal completely bounded maps from $M$ to $N$ will be denoted $\mathcal{C B}^{\sigma}(M, N)$. If $X$ is subspace of $M$, and $\mathcal{A}$ is a subalgebra of $M$, we denote by $\mathcal{C B}_{\mathcal{A}}^{\sigma, X}(M)$ the algebra of normal completely bounded $\mathcal{A}$-bimodule maps on $M$ that leave $X$ globally invariant, that is, those completely bounded maps $\Phi: M \rightarrow M$ that satisfy $\Phi(a x b)=a \Phi(x) b$, for $x \in M, a, b \in \mathcal{A}$ and $\Phi(X) \subseteq X$.

Since every bounded linear functional $\varphi: X \rightarrow \mathbb{C}$ is completely bounded with 
$\|\varphi\|_{c b}=\|\varphi\|\left[80\right.$, Proposition 3.8], we have $X^{*}=\mathcal{C B}(X, \mathbb{C})$, isometrically, and the identification $M_{n}\left(X^{*}\right)=\mathcal{C B}\left(X, M_{n}(\mathbb{C})\right), n \in \mathbb{N}$, turns $X^{*}$ into an operator space. In particular, norm closed subspaces of $X^{*}$ are operator spaces.

Let $\left(X_{i}\right)_{i \in I}$ be a collection of operator spaces. The direct sum $\oplus_{i \in I} X_{i}$ becomes an operator space under the matrix norm

$$
\|x\|_{M_{n}\left(\oplus_{i \in I} X_{i}\right)}=\sup _{i \in I}\left\|x_{i}\right\|_{M_{n}\left(X_{i}\right)}, \quad x=\oplus_{i \in I} x_{i} \in M_{n}\left(\oplus_{i \in I} X_{i}\right), n \in \mathbb{N} .
$$

There is an associated operator space structure on the predual $\left(\oplus_{i \in I} X_{i}^{*}\right)_{*}$ which will be denoted $\oplus_{1}\left\{X_{i} \mid i \in I\right\}$.

In general, we have the following duality relations for a linear map $\Phi: X \rightarrow Y$ (see [29, Theorem 4.1.8]):

- $\Phi$ is a complete quotient map $\Leftrightarrow \Phi^{*}$ is a complete isometry;

- $\Phi$ is a complete isometry $\Leftrightarrow \Phi^{*}$ is a complete quotient map $\Leftrightarrow \Phi^{*}$ is an exact complete quotient map.

If $X$ and $Y$ are operator spaces, given $u \in M_{n}(X \otimes Y)$, we define

$$
\|u\|_{\wedge}=\inf \{\|\alpha\|\|x\|\|y\|\|\beta\|: u=\alpha(x \otimes y) \beta\}
$$

where the infimum is taken over all possible decompositions with $x \in M_{p}(X), y \in$ $M_{q}(Y), \alpha \in M_{n, p q}(\mathbb{C}), \beta \in M_{p q, n}(\mathbb{C})$ and $p, q \in \mathbb{N}$. One may verify that this is indeed an operator space matrix norm. The completion of the algebraic tensor product $X \otimes Y$ under this matrix norm becomes an operator space, called the operator space projective tensor product, denoted by $X \widehat{\otimes} Y$. Analogously to the Banach space setting, we have the following completely isometric isomorphisms

$$
\mathcal{C B}\left(X, Y^{*}\right) \cong(X \widehat{\otimes} Y)^{*} \cong \mathcal{C B}\left(Y, X^{*}\right)
$$

via the identification $\langle\Phi, x \otimes y\rangle:=\langle\Phi(x), y\rangle$ for all $\Phi \in \mathcal{C B}\left(X, Y^{*}\right), x \in X$ and $y \in Y$, 
and similarly for $\Phi \in \mathcal{C B}\left(Y, X^{*}\right)$.

Given operator spaces $X_{i}$ and $Y_{i}$ for $i=1,2$ and completely bounded maps $\Phi$ : $X_{1} \rightarrow X_{2}$ and $\Psi: Y_{1} \rightarrow Y_{2}$, the map $\Phi \otimes \Psi: X_{1} \otimes Y_{1} \rightarrow X_{2} \otimes Y_{2}$ extends uniquely to a completely bounded map (still denoted) $\Phi \otimes \Psi: X_{1} \widehat{\otimes} Y_{1} \rightarrow X_{2} \widehat{\otimes} Y_{2}$ with $\|\Phi \otimes \Psi\| \leq$ $\|\Phi\|_{c b}\|\Psi\|_{c b}[29$, Corollary 7.1.3].

If $M$ and $N$ are von Neumann algebras acting on Hilbert spaces $H$ and $K$, respectively, then the algebraic tensor product $M \otimes N$ acts naturally on $H \otimes_{2} K$, and its weak* closure in $\mathcal{B}\left(H \otimes_{2} K\right)$ is a von Neumann algebra, called the von Neumann algebraic tensor product of $M$ and $N$, denoted $M \bar{\otimes} N$. It can be shown that this definition is independent of the choice of Hilbert spaces [100, §4.5]. At the level of preduals, there is a fundamental relationship with the operator space projective tensor product [29, Theorem 7.2.4]:

$$
(M \bar{\otimes} N)_{*} \cong M_{*} \widehat{\otimes} N_{*},
$$

completely isometrically.

If $M_{i}$ and $N_{i}$ are von Neumann algebras for $i=1,2$ and $\Phi: M_{1} \rightarrow M_{2}$ and $\Psi: N_{1} \rightarrow N_{2}$ are normal completely bounded maps, then the map $\Phi \otimes \Psi: M_{1} \otimes$ $N_{1} \rightarrow M_{2} \otimes N_{2}$ extends uniquely to a normal completely bounded map from $M_{1} \bar{\otimes} N_{1}$ to $M_{2} \bar{\otimes} N_{2}$. In particular, if $N_{1}=N_{2}=N$ and $\Psi=$ id is the identity on $N$, then $\Phi \otimes$ id : $M_{1} \otimes N \rightarrow M_{2} \otimes N$ extends uniquely to an element (still denoted) $\Phi \otimes$ id $\in \mathcal{C B}^{\sigma}\left(M_{1} \bar{\otimes} N, M_{2} \bar{\otimes} N\right)$. More generally, if $\Phi \in \mathcal{C B}\left(M_{1}, M_{2}\right)$ is not necessarily normal, there exists an amplification to an element $\Phi \otimes$ id $\in \mathcal{C B}\left(M_{1} \bar{\otimes} N, M_{2} \bar{\otimes} N\right)$ which is defined by

$$
\langle(\Phi \otimes \operatorname{id})(X), \tau \otimes \omega\rangle=\langle\Phi((\operatorname{id} \otimes \omega)(X)), \tau\rangle, \quad X \in M_{1} \bar{\otimes} N, \tau \in\left(M_{2}\right)_{*}, \omega \in N_{*} .
$$

For details on this amplification procedure we refer the reader to [75]. An important property that we shall use frequently without comment is the following commutation 
relation (see [75, Theorem 5.1]): if $\Phi \in \mathcal{C B}\left(M_{1}, M_{2}\right)$ and $\Psi \in \mathcal{C B}^{\sigma}\left(N_{1}, N_{2}\right)$, then

$$
\left(\Phi \otimes \operatorname{id}_{N_{2}}\right) \circ\left(\operatorname{id}_{M_{1}} \otimes \Psi\right)=\left(\operatorname{id}_{M_{2}} \otimes \Psi\right) \circ\left(\Phi \otimes \operatorname{id}_{N_{1}}\right) \in \mathcal{C} \mathcal{B}\left(M_{1} \bar{\otimes} N_{1}, M_{2} \bar{\otimes} N_{2}\right) .
$$

If $M \subseteq \mathcal{B}(H)$ is a von Neumann algebra, then $M$ is said to be injective if there exists a projection of norm one $E: \mathcal{B}(H) \rightarrow M$. By a classical result of Tomiyama [105], any such projection is automatically a completely positive $M$-bimodule map, and is therefore called a conditional expectation. The definition of injectivity is independent of the faithfully representing Hilbert space, and moreover, it is equivalent to 1-injectivity in the category of operator spaces and completely bounded maps (see §2.5). One of the major results in operator algebras relates injectivity with the following approximation property for $C^{*}$-algebras.

Definition 2.4.4. A $C^{*}$-algebra $\mathcal{A}$ is nuclear if there exist nets of completely positive contractions $\Phi_{i}: \mathcal{A} \rightarrow M_{n_{i}}(\mathbb{C})$ and $\Psi_{i}: M_{n_{i}}(\mathbb{C}) \rightarrow \mathcal{A}$ such that $\Psi_{i} \circ \Phi_{i}$ converges to $\operatorname{id}_{\mathcal{A}}$ in the point-norm topology, that is,

$$
\left\|\Psi_{i} \circ \Phi_{i}(x)-x\right\| \rightarrow 0, \quad x \in \mathcal{A} .
$$

For any $C^{*}$-algebra $\mathcal{A}$, it is well-known that its second dual $\mathcal{A}^{* *}$ is a von Neumann algebra under the left (= right) Arens product [86, Theorem 2.11.2]. In fact, $\mathcal{A}^{* *}$ is the universal enveloping von Neumann algebra of $\mathcal{A}$ [100, Theorem III.2.4]. Through the ground-breaking work of Connes [15], Choi-Effros [13], and Effros-Lance [28], it was shown that $\mathcal{A}$ is nuclear if and only if $\mathcal{A}^{* *}$ is injective.

\subsection{Operator Modules and Homology}

Definition 2.5.1. Let $\mathcal{A}$ be an operator space which has the structure of a Banach algebra. Then $\mathcal{A}$ is a completely contractive Banach algebra if the multiplication map extends to a complete contraction $m_{\mathcal{A}}: \mathcal{A} \widehat{\otimes} \mathcal{A} \rightarrow \mathcal{A}$. We say that an operator space 
$X$ a right operator $\mathcal{A}$-module if it is a right Banach $\mathcal{A}$-module such that the module map $m_{X}: X \widehat{\otimes} \mathcal{A} \rightarrow X$ is completely contractive. This is equivalent to the following condition:

$$
\left\|\left[x_{i j} \cdot a_{k l}\right]\right\|_{m n} \leq\left\|\left[x_{i j}\right]\right\|_{m}\left\|\left[a_{k l}\right]\right\|_{n}
$$

for all $\left[x_{i j}\right] \in M_{m}(X),\left[a_{k l}\right] \in M_{n}(\mathcal{A})$ and $m, n \in \mathbb{N}$. We say that $X$ is

- essential if $\langle X \cdot \mathcal{A}\rangle=X$, where $\langle\cdot\rangle$ denotes the closed linear span;

- faithful if for every non-zero $x \in X$, there is $a \in \mathcal{A}$ such that $x \cdot a \neq 0$.

We denote by $\bmod -\mathcal{A}$ the category of right operator $\mathcal{A}$-modules with morphisms given by completely bounded module homomorphisms. If $X \in \bmod -\mathcal{A}$ is a dual operator space such that the action of $a$ is weak* continuous for all $a \in \mathcal{A}$, then we say that $X$ is a dual operator $\mathcal{A}$-module. We let $\operatorname{nmod}-\mathcal{A}$ denote the category of dual right operator $\mathcal{A}$-modules with morphisms given by weak*-weak* continuous completely bounded module homomorphisms (the $\mathbf{n}$ standing for normal, as usual).

Left operator $\mathcal{A}$-modules and operator $\mathcal{A}$-bimodules are defined similarly, along with the analogous notions of essentiality and faithfulness. We denote the respective categories by $\mathcal{A}-\bmod$ and $\mathcal{A}-\bmod -\mathcal{A}$. Throughout this section we will mainly restrict ourselves to right operator modules and leave the corresponding notions and results for left/bimodules to the reader.

Remark 2.5.2. Regarding terminology, in what follows we will often omit the term "operator" when discussing homological properties of operator modules as we will be working exclusively in the operator space category.

Let $\mathcal{A}$ be a completely contractive Banach algebra, $X \in \bmod -\mathcal{A}$ and $Y \in$ $\mathcal{A}$-mod. The $\mathcal{A}$-module tensor product of $X$ and $Y$ is the quotient space $X \widehat{\otimes}_{\mathcal{A}} Y:=$ $X \widehat{\otimes} Y / N$, where

$$
N=\langle x \cdot a \otimes y-x \otimes a \cdot y \mid x \in X, y \in Y, a \in \mathcal{A}\rangle .
$$


Analogously to the isomorphism (2.3), it follows that

$$
\mathcal{C B}_{\mathcal{A}}\left(X, Y^{*}\right) \cong N^{\perp} \cong\left(X \widehat{\otimes}_{\mathcal{A}} Y\right)^{*}
$$

where $\mathcal{C B}_{\mathcal{A}}\left(X, Y^{*}\right)$ denotes the space of completely bounded right $\mathcal{A}$-module maps $\Phi: X \rightarrow Y^{*}$. If $Y=\mathcal{A}$, then clearly $N \subseteq \operatorname{Ker}\left(m_{X}\right)$ where $m_{X}: X \widehat{\otimes} \mathcal{A} \rightarrow X$ is the module map. If the induced mapping $\widetilde{m}_{X}: X \widehat{\otimes}_{\mathcal{A}} \mathcal{A} \rightarrow X$ is a completely isometric isomorphism we say that $X$ is an induced right $\mathcal{A}$-module. A similar definition applies for left modules. In particular, we say that a completely contractive Banach algebra $\mathcal{A}$ is self-induced if $\widetilde{m}_{\mathcal{A}}: \mathcal{A} \widehat{\otimes}_{\mathcal{A}} \mathcal{A} \cong \mathcal{A}$, completely isometrically.

Let $\mathcal{A}$ be a completely contractive Banach algebra and $X \in \bmod -\mathcal{A}$. The identification $\mathcal{A}^{+}=\mathcal{A} \oplus_{1} \mathbb{C}$ turns the unitization of $\mathcal{A}$ into a unital completely contractive Banach algebra, and it follows that $X$ becomes a right operator $\mathcal{A}^{+}$module via the extended action

$$
x \cdot(a+\lambda e)=x \cdot a+\lambda x, \quad a \in \mathcal{A}^{+}, \lambda \in \mathbb{C}, x \in X .
$$

Let $C \geq 1$. We say that $X$ is relatively $C$-projective if there exists a morphism $\Phi: X \rightarrow X \widehat{\otimes} \mathcal{A}^{+}$satisfying $\|\Phi\|_{c b} \leq C$ which is a right inverse to the extended multiplication map $m_{X}^{+}: X \widehat{\otimes} \mathcal{A}^{+} \rightarrow X$. We say that $X$ is $C$-projective if for every $Y, Z \in \bmod -\mathcal{A}$, every complete quotient morphism $\Phi: Y \rightarrow Z$, every morphism $\Psi: X \rightarrow Z$, and every $\varepsilon>0$, there exists a morphism $\widetilde{\Psi}_{\varepsilon}: X \rightarrow Y$ such that $\left\|\widetilde{\Psi}_{\varepsilon}\right\|_{c b}<C\|\Psi\|_{c b}+\varepsilon$ and $\Phi \circ \widetilde{\Psi}_{\varepsilon}=\Psi$, that is, the following diagram commutes:

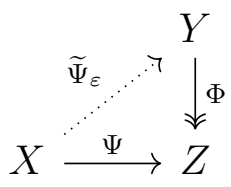

For example, any unital completely contractive Banach algebra $\mathcal{A}$ is $\|e\|$-projective over itself, as in the above situation one simply defines $\widetilde{\Psi}_{\varepsilon}(a)=y_{\varepsilon} \cdot a, a \in \mathcal{A}$, where $y_{\varepsilon} \in Y$ satisfies $\Phi\left(y_{\varepsilon}\right)=\Psi(e)$ and $\left\|y_{\varepsilon}\right\|<\|\Psi(e)\|+\varepsilon$. When $\mathcal{A}=\mathbb{C}$, the definition 
of $C$-projectivity coincides with that of a $C$-projective operator space [8, Definition 3.3].

The dual notion of projectivity, which arises by reversing the arrows in the above diagram, is injectivity, and is a central concept in this thesis. If $X, Y \in \bmod -\mathcal{A}$, an injective morphism $\Phi: X \rightarrow Y$ is said to be admissible if there exists a completely bounded map (not necessarily a morphism) $\Phi^{-1}: Y \rightarrow X$ such that $\Phi^{-1} \circ \Phi=$ $\operatorname{id}_{X}$. For $C \geq 1$, we say that $X$ is $C$-injective (respectively, relatively $C$-injective) if for every $Y, Z \in \bmod -\mathcal{A}$, every completely isometric (respectively, completely isometric admissible) morphism $\Phi: Y \hookrightarrow Z$, and every morphism $\Psi: Y \rightarrow X$, there exists a morphism $\widetilde{\Psi}: Z \rightarrow X$ such that $\|\widetilde{\Psi}\|_{c b} \leq C\|\Psi\|_{c b}$ (respectively, $\|\widetilde{\Psi}\|_{c b} \leq$ $C\|\Psi\|_{c b}\left\|\Phi^{-1}\right\|_{c b}$ ) and $\widetilde{\Psi} \circ \Phi=\Psi$, i.e., the following diagram commutes:

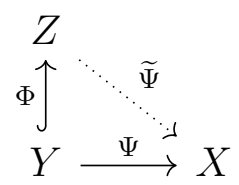

For example, the dual $X^{*}$ of any $X \in \bmod -\mathcal{A}$ has a canonical left $\mathcal{A}$-module structure, and $X^{*}$ is $C$-injective in $\mathcal{A}-\bmod$ whenever $X$ is $C$-projective in $\bmod -\mathcal{A}$ by the module version of $[8$, Theorem 3.5]. Also, $X \in \bmod -\mathcal{A}$ is relatively $C$ projective if and only if $X^{*}$ is relatively $C$-injective in $\mathcal{A}-\operatorname{nmod}$ (see Proposition 4.3.3), where, naturally, the notion of admissibility in $\mathcal{A}-\mathbf{n m o d}$ is with respect to weak* continuous inverses.

The celebrated Arveson-Wittstock extension theorem says that $\mathcal{B}(H)$ is 1-injective in $\bmod -\mathbb{C}$ for any Hilbert space $H[3,115]$, that is, 1-injective in the category of operator spaces and completely bounded maps.

In Chapter 4 we will need a predual characterization of 1-injectivity for dual operator modules. With this in mind, let $\mathcal{A}$ be a completely contractive Banach algebra and let $X \in \mathcal{A}-\bmod$. We say that $X$ is a 1 -flat in $\mathcal{A}-\bmod$ if $X^{*}$ is 1 -injective in $\bmod -\mathcal{A}$. It is easily seen that 1 -injectivity of $X^{*}$ is equivalent to the 
following condition: for every 1 -exact sequence of right $\mathcal{A}$-modules

$$
0 \rightarrow Y \hookrightarrow Z \rightarrow Z / Y \rightarrow 0
$$

the induced sequence

$$
0 \rightarrow \mathcal{C B}_{\mathcal{A}}\left(Z / Y, X^{*}\right) \hookrightarrow \mathcal{C B}_{\mathcal{A}}\left(Z, X^{*}\right) \rightarrow \mathcal{C B}_{\mathcal{A}}\left(Y, X^{*}\right) \rightarrow 0
$$

is 1-exact, where 1-exactness refers to an exact sequence such that the injection $(\hookrightarrow)$ is a complete isometry and the surjection $(\rightarrow)$ is a complete quotient map. Taking the pre-adjoint of the above sequence we obtain the following sequence

$$
0 \rightarrow Y \widehat{\otimes}_{\mathcal{A}} X \hookrightarrow Z \widehat{\otimes}_{\mathcal{A}} X \rightarrow Z / Y \widehat{\otimes}_{\mathcal{A}} X \rightarrow 0 .
$$

Since a short sequence is 1-exact if and only if its dual sequence is 1-exact, it follows that $X$ is 1-flat if and only if the functor $(\cdot) \widehat{\otimes}_{\mathcal{A}} X$ is 1-exact, i.e., it maps 1-exact sequences of right $\mathcal{A}$-modules to 1 -exact sequences of operator spaces. Naturally, there are notions of $C$-flatness but we will only be concerned with $C=1$ (see [114] for details).

Remark 2.5.3. Our notions of projectivity and injectivity are closer in spirit to the approach taken in operator space theory (cf. [8]) and the recent approach of Helemskii (cf. [44]) rather than Banach algebra homology, where the related notions are usually studied solely from the relative perspective, and allow for liftings (respectively, extensions) via admissible morphisms that are not necessarily complete quotients (respectively, complete isometries). 


\section{Chapter 3}

\section{Locally Compact Quantum Groups}

The quantization of abstract harmonic analysis was established over half a century culminating in the early seventies with the theory of Kac algebras [30]. This wider category contains that of locally compact groups and provides the general framework necessary to establish Pontrjagin duality.

Shortly afterwards, similar structures were appearing in algebra and mathematical physics, and the concept of a "quantum group" emerged. This resulted in many different axiomatic approaches, but from a harmonic analysis perspective, the most natural are the von Neumann algebraic locally compact quantum groups, as developed by Kustermans and Vaes $[63,64]$. This chapter is devoted to a brief introduction to this theory. We refer the reader to $[63,64,106]$ for details and proofs.

\section{1 von Neumann Algebraic Quantum Groups}

Definition 3.1.1. A Hopf-von Neumann algebra is a pair $(M, \Gamma)$ where $M$ is a von Neumann algebra and $\Gamma: M \rightarrow M \bar{\otimes} M$ is a normal, unital, injective *-homomorphism satisfying the co-associativity condition $(\mathrm{id} \otimes \Gamma) \Gamma=(\Gamma \otimes \mathrm{id}) \Gamma$, that is, the following 
diagram commutes.

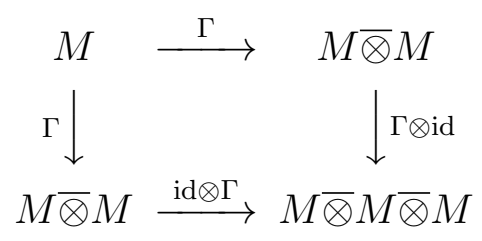

$\Gamma$ is then called a co-multiplication.

Given a Hopf-von Neumann algebra $(M, \Gamma)$, the preadjoint $\Gamma_{*}: M_{*} \widehat{\otimes} M_{*} \rightarrow M_{*}$ of the co-multiplication induces an associative completely contractive multiplication on $M_{*}$, turning it into a completely contractive Banach algebra.

Definition 3.1.2. A locally compact quantum group $\mathbb{G}$ is a quadruple $\mathbb{G}=(M, \Gamma, \varphi, \psi)$ where:

1. $(M, \Gamma)$ is a Hopf-von Neumann algebra;

2. $\varphi$ is an n.s.f. weight on $M$, called the left Haar weight, satisfying:

$$
\varphi((\omega \otimes \mathrm{id}) \Gamma(x))=\omega(1) \varphi(x), \quad x \in \mathcal{M}_{\varphi}, \omega \in M_{*} ;
$$

3. $\psi$ is an n.s.f. weight on $M$, called the right Haar weight, satisfying:

$$
\psi((\operatorname{id} \otimes \omega) \Gamma(x))=\omega(1) \psi(x), \quad x \in \mathcal{M}_{\psi}, \omega \in M_{*} .
$$

If $\mathbb{G}=(M, \Gamma, \varphi, \psi)$ is a locally compact quantum group, we say that $\mathbb{G}$ is commutative if $M$ is commutative, and co-commutative if $\Gamma$ is symmetric, that is, $\Sigma \circ \Gamma=\Gamma$ where $\Sigma: M \bar{\otimes} M \rightarrow M \bar{\otimes} M$ is the flip map given by $\Sigma(x \otimes y)=y \otimes x, x, y \in M$. Two quantum groups $\mathbb{G}_{1}$ and $\mathbb{G}_{2}$ are isomorphic if there exists a von Neumann algebraic isomorphism $\alpha: M_{1} \rightarrow M_{2}$ such that $\Gamma_{2} \circ \alpha=(\alpha \otimes \alpha) \circ \Gamma_{1}$.

Example 3.1.3. Let $G$ be a locally compact group, and define $\Gamma_{a}: L^{\infty}(G) \rightarrow$ $L^{\infty}(G) \bar{\otimes} L^{\infty}(G) \cong L^{\infty}(G \times G)$ by

$$
\Gamma_{a}(h)(s, t)=h(s t), \quad h \in L^{\infty}(G), s, t \in G .
$$


Then $\Gamma_{a}$ is a co-multiplication which turns $\left(L^{\infty}(G), \Gamma_{a}\right)$ into a Hopf-von Neumann algebra. If $\varphi_{a}$ is the weight defined by the left Haar measure $d t$, then for $f \in L^{1}(G)=$ $L^{\infty}(G)_{*}$ and $h \in L^{\infty}(G) \cap L^{1}(G)=\mathcal{M}_{\varphi_{a}}$, we have

$$
\begin{aligned}
\varphi_{a}\left((f \otimes \mathrm{id}) \Gamma_{a}(h)\right) & =\int_{G}(f \otimes \mathrm{id}) \Gamma_{a}(h)(t) d t \\
& =\int_{G} \int_{G} f(s) h(s t) d s d t .
\end{aligned}
$$

Since $h \in \mathcal{M}_{\varphi_{a}}$, we may apply Fubini's theorem to obtain:

$$
\begin{aligned}
\varphi_{a}\left((f \otimes \mathrm{id}) \Gamma_{a}(h)\right) & =\int_{G} f(s) \int_{G} h(s t) d t d s \\
& =\int_{G} f(s) \int_{G} h(t) d t d s \\
& =\langle f, 1\rangle \varphi_{a}(h) .
\end{aligned}
$$

Thus, $\varphi_{a}$ is a left Haar weight on $L^{\infty}(G)$. Similarly, the weight $\psi_{a}$ given by the right Haar measure is a right Haar weight, and $\mathbb{G}_{a}=\left(L^{\infty}(G), \Gamma_{a}, \varphi_{a}, \psi_{a}\right)$ is a commutative locally compact quantum group. The co-multiplication $\Gamma_{a}$ is the dual of convolution in $L^{1}(G)$, viewed as a map * : $L^{1}(G) \widehat{\otimes} L^{1}(G) \rightarrow L^{1}(G)$.

As expected, we see that every locally compact group $G$ generates a "classical" locally compact quantum group given by the commutative von Neumann algebra $L^{\infty}(G)$. In fact, these are all such quantum groups.

Theorem 3.1.4. [109, §2] Let $\mathbb{G}=(M, \Gamma, \varphi, \psi)$ be a commutative locally compact quantum group. Then there exists a unique locally compact group $G$ for which $\mathbb{G} \cong$ $\mathbb{G}_{a}=\left(L^{\infty}(G), \Gamma_{a}, \varphi_{a}, \psi_{a}\right)$.

Example 3.1.5. Let $G$ be a locally compact group. The map $\Gamma_{s}$ given on generators of $\mathcal{L}(G)$ by

$$
\Gamma_{s}(\lambda(t))=\lambda(t) \otimes \lambda(t), \quad t \in G,
$$

can be extended to a symmetric co-multiplication $\Gamma_{s}: \mathcal{L}(G) \rightarrow \mathcal{L}(G) \bar{\otimes} \mathcal{L}(G)$. It 
follows that there exists an n.s.f. weight $\varphi_{s}$ on $\mathcal{L}(G)$ (unique up to a positive scalar), namely Haagerup's Plancherel weight, which is both a left and a right Haar weight with respect to $\Gamma_{s}\left([100], \S\right.$ VII.3). Thus, $\mathbb{G}_{s}=\left(\mathcal{L}(G), \Gamma_{s}, \varphi_{s}\right)$ is a co-commutative locally compact quantum group. The co-multiplication $\Gamma_{s}$ is the dual of pointwise multiplication in $A(G)$, viewed as a map $\cdot: A(G) \widehat{\otimes} A(G) \rightarrow A(G)$.

Locally compact quantum groups therefore provide a sufficient framework for studying the duality of non-abelian groups, that is, the duality between $L^{\infty}(G)$ and $\mathcal{L}(G)$. Consequently, there is a dual version of Theorem 3.1.4. The proof can be established using theory we will encounter in $\S 3.3$, along with [109, §2].

Theorem 3.1.6. Let $\mathbb{G}=(M, \Gamma, \varphi, \psi)$ be a co-commutative locally compact quantum group. Then there exists a unique locally compact group $G$ for which $\mathbb{G} \cong \mathbb{G}_{s}=$ $\left(\mathcal{L}(G), \Gamma_{s}, \varphi_{s}\right)$.

Motivated by the above examples, we may generalize various concepts from abstract harmonic analysis to the setting of locally compact quantum groups. Firstly, note that the existence of Haar weights is assumed in the definition of a locally compact quantum group. To find an axiomatization which yields the existence is a major open problem in quantum group theory. Despite this fact, assuming existence is enough to guarantee uniqueness.

Theorem 3.1.7. [106, §1.10] The left and right Haar weights on a locally compact quantum group are unique up to positive scalars.

Remark 3.1.8. Under the GNS construction of the left (respectively, right) Haar weight, we may identify $M$ with a subalgebra in $\mathcal{B}\left(H_{\varphi}\right)$ (respectively, $\mathcal{B}\left(H_{\psi}\right)$ ) and we will do so without comment throughout the thesis.

For notational symmetry, given a locally compact quantum group $\mathbb{G}=(M, \Gamma, \varphi, \psi)$, we write $L^{\infty}(\mathbb{G}):=M$ and $L^{1}(\mathbb{G}):=M_{*}$. In the commutative (respectively, cocommutative) setting we simply have $L^{\infty}\left(\mathbb{G}_{a}\right)=L^{\infty}(G)$ and $L^{1}\left(\mathbb{G}_{a}\right)=L^{1}(G)$ (respectively, $L^{\infty}\left(\mathbb{G}_{s}\right)=\mathcal{L}(G)$ and $\left.L^{1}\left(\mathbb{G}_{s}\right)=A(G)\right)$ for a locally compact group $G$. For 
the remainder of the thesis we will refrain from writing the quadruple notation for a locally compact quantum group and simply write $\mathbb{G}$, where the usual notations for the co-multiplication and Haar weights will be understood.

Analogously to the group setting, a locally compact quantum group $\mathbb{G}$ is said to be discrete if $L^{1}(\mathbb{G})$ is a unital Banach algebra and compact if $\varphi$ is finite.

If $X$ is an operator system in $L^{\infty}(\mathbb{G})$ that is also a right $L^{1}(\mathbb{G})$-submodule, then a left invariant mean on $X$ is a state $m \in X^{*}$ satisfying

$$
\langle m, x \star f\rangle=\langle f, 1\rangle\langle m, x\rangle \quad x \in X, f \in L^{1}(\mathbb{G}) .
$$

Right and two-sided invariant means are defined similarly. A locally compact quantum group $\mathbb{G}$ is said to be amenable if there exists a left invariant mean on $L^{\infty}(\mathbb{G})$. It is known that $\mathbb{G}$ is amenable if and only if there exists a right (equivalently, two-sided) invariant mean on $L^{\infty}(\mathbb{G})$ (cf. [26, Proposition 3]). We say that $\mathbb{G}$ is co-amenable if $L^{1}(\mathbb{G})$ has a bounded left (equivalently, right or two-sided) approximate identity (cf. [6, Theorem 3.1]). Commutative quantum groups $\mathbb{G}_{a}$ are always co-amenable and are amenable precisely when the underlying group is amenable. Dually, co-commutative quantum groups $\mathbb{G}_{s}$ are always amenable [85, Theorem 4], and are co-amenable if and only if the underlying is amenable, by Leptin's classical theorem [68]. In Example 3.6.1 we will encounter examples of amenable and co-amenable quantum groups not arising from groups. We also mention that there is a general procedure for constructing non-commutative and non-co-commutative examples of locally compact quantum groups with desirable properties, such as amenability, known as the bi-crossed product construction $[70,109]$.

An important fact that we will use several times throughout the thesis is that $L^{1}(\mathbb{G})$ is always self-induced. The proof follows from a simple application of $[107$, Theorem 2.7], but we provide the details for the convenience of the reader.

Proposition 3.1.9. Let $\mathbb{G}$ be a locally compact quantum group. Then $L^{1}(\mathbb{G})$ is a self-induced completely contractive Banach algebra. 
Proof. Let $\widetilde{m}: L^{1}(\mathbb{G}) \widehat{\otimes}_{L^{1}(\mathbb{G})} L^{1}(\mathbb{G}) \rightarrow L^{1}(\mathbb{G})$ be the induced multiplication map. Then $\widetilde{m}^{*}: L^{\infty}(\mathbb{G}) \rightarrow\left(L^{1}(\mathbb{G}) \widehat{\otimes}_{L^{1}(\mathbb{G})} L^{1}(\mathbb{G})\right)^{*}$ is nothing but the co-multiplication $\Gamma$. Since $\left(L^{1}(\mathbb{G}) \widehat{\otimes}_{L^{1}(\mathbb{G})} L^{1}(\mathbb{G})\right)^{*} \cong N^{\perp}$, where $N \subseteq L^{1}(\mathbb{G}) \widehat{\otimes} L^{1}(\mathbb{G})$ is the closed linear span of $\left\{f \star g \otimes h-f \otimes g \star h \mid f, g, h \in L^{1}(\mathbb{G})\right\}$, given $X \in\left(L^{1}(\mathbb{G}) \widehat{\otimes}_{L^{1}(\mathbb{G})} L^{1}(\mathbb{G})\right)^{*} \subseteq$ $L^{\infty}(\mathbb{G}) \bar{\otimes} L^{\infty}(\mathbb{G})$, it follows that $(\Gamma \otimes \mathrm{id})(X)=(\mathrm{id} \otimes \Gamma)(X)$. Hence, $X \in \Gamma\left(L^{\infty}(\mathbb{G})\right)$ by [107, Theorem 2.7], and $\widetilde{m}^{*}$ is surjective. Since $\widetilde{m}^{*}=\Gamma$ is also a complete isometry, the result follows.

\subsection{The Fundamental Unitary}

Given a locally compact group $G$, there is a fundamental unitary operator $W_{a}$ : $L^{2}(G \times G) \rightarrow L^{2}(G \times G)$ given by

$$
W_{a} \xi(s, t)=\xi\left(s, s^{-1} t\right), \quad s, t \in G,
$$

for all $\xi \in L^{2}(G \times G) \cong L^{2}(G) \otimes_{2} L^{2}(G)$. We use the adjective "fundamental" as this unitary carries nearly all the information of $G$ along with its corresponding quantum group $\mathbb{G}_{a}$ in the following sense:

1. $\Gamma_{a}\left(M_{h}\right)=W_{a}^{*}\left(1 \otimes M_{h}\right) W_{a}, h \in L^{\infty}(G)$;

2. $W_{a} \in L^{\infty}(G) \bar{\otimes} \mathcal{L}(G)=L^{\infty}(G) \bar{\otimes} \mathcal{R}(G)^{\prime}$;

3. $L^{\infty}(G)=\overline{\left\{(\operatorname{id} \otimes \psi) W_{a}: \psi \in A(G)\right.}{ }^{*}$;

4. $C_{0}(G)={\overline{\left\{(\mathrm{id} \otimes \psi) W_{a}: \psi \in A(G)\right.}}^{\|\cdot\|}$;

5. $\lambda(f)=(f \otimes \mathrm{id})\left(W_{a}\right), f \in L^{1}(G)$;

6. $\left.\mathcal{L}(G)=\overline{\left\{(f \otimes \mathrm{id}) W_{a}: f \in L^{1}(G)\right.}\right\}^{w^{*}} ;$

7. $\left.C_{\lambda}^{*}(G)=\overline{\left\{(f \otimes \mathrm{id}) W_{a}: f \in L^{1}(G)\right.}\right\}^{\|\cdot\|}$. 
In general, given a locally compact quantum group $\mathbb{G}$, there exists a unitary operator $W: H_{\varphi} \otimes_{2} H_{\varphi} \rightarrow H_{\varphi} \otimes_{2} H_{\varphi}$ satisfying

$$
W^{*}\left(\Lambda_{\varphi}(x) \otimes \Lambda_{\varphi}(y)\right)=\left(\Lambda_{\varphi} \otimes \Lambda_{\varphi}\right)(\Gamma(y)(x \otimes 1)), \quad x, y \in \mathcal{N}_{\varphi} .
$$

This operator satisfies the pentagonal relation, that is

$$
W_{12} W_{13} W_{23}=W_{23} W_{12},
$$

where $W_{12}=W \otimes 1, W_{23}=1 \otimes W, W_{13}=(\sigma \otimes 1) W_{23}(\sigma \otimes 1)$ and $\sigma$ is the flip map on $H_{\varphi} \otimes_{2} H_{\varphi}$. The map $W$ is therefore called a multiplicative unitary, and similarly to the classical case above, it carries nearly all the information about $\mathbb{G}$. In particular, the co-multiplication on $L^{\infty}(\mathbb{G})$ may be written as

$$
\Gamma(x)=W^{*}(1 \otimes x) W, \quad x \in L^{\infty}(\mathbb{G}) .
$$

The operator $W$ is called the left fundamental unitary of $\mathbb{G}$.

Definition 3.2.1. The left regular representation $\lambda: L^{1}(\mathbb{G}) \rightarrow \mathcal{B}\left(H_{\varphi}\right)$ of $\mathbb{G}$ is defined by

$$
\lambda(f)=(f \otimes \mathrm{id})(W), \quad f \in L^{1}(\mathbb{G}) .
$$

Using the properties of $W$, it can be shown that $\lambda$ is an injective completely contractive homomorphism.

In a similar fashion, there is a right fundamental unitary operator $V: H_{\psi} \otimes_{2} H_{\psi} \rightarrow$ $H_{\psi} \otimes_{2} H_{\psi}$ defined by

$$
V\left(\Lambda_{\psi}(x) \otimes \Lambda_{\psi}(y)\right)=\left(\Lambda_{\psi} \otimes \Lambda_{\psi}\right)(\Gamma(x)(1 \otimes y)), \quad x, y, \in \mathcal{N}_{\psi}
$$


It can be shown that $V$ also satisfies the pentagonal relation:

$$
V_{12} V_{13} V_{23}=V_{23} V_{12}
$$

and that the co-multiplication on $L^{\infty}(\mathbb{G})$ may be written as

$$
\Gamma(x)=V(x \otimes 1) V^{*}, \quad x \in L^{\infty}(\mathbb{G}) .
$$

We also obtain the right regular representation $\rho: L^{1}(\mathbb{G}) \rightarrow \mathcal{B}\left(H_{\psi}\right)$, given by

$$
\rho(f)=(\mathrm{id} \otimes f)(V), \quad f \in L^{1}(\mathbb{G}) .
$$

\subsection{Duality}

One of the many triumphs of Fourier analysis on locally compact abelian groups provided the following isomorphisms:

$$
L^{\infty}(G) \cong \mathcal{L}(\widehat{G}), \quad L^{1}(G) \cong A(\widehat{G}), \quad \text { and } \quad L^{2}(G) \cong L^{2}(\widehat{G})
$$

In order to achieve a successful generalization, we must therefore ensure the duality of $\mathbb{G}_{a}=\left(L^{\infty}(G), \Gamma_{a}, \varphi_{a}, \psi_{a}\right)$ and $\mathbb{G}_{s}=\left(\mathcal{L}(G), \Gamma_{s}, \varphi_{s}\right)$ for all locally compact groups. This may be done with the aid of the fundamental unitaries introduced above.

Theorem 3.3.1 (The Dual Quantum Group). [106, $\S 1.11]$ Let $\mathbb{G}$ be a locally compact quantum group with left fundamental unitary $W$. Define $L^{\infty}(\widehat{\mathbb{G}})=\{\lambda(f): f \in$ $\left.L^{1}(\mathbb{G})\right\}^{\prime \prime}$. Then $\widehat{\Gamma}: L^{\infty}(\widehat{\mathbb{G}}) \rightarrow L^{\infty}(\widehat{\mathbb{G}}) \bar{\otimes} L^{\infty}(\widehat{\mathbb{G}})$ given by

$$
\widehat{\Gamma}(\hat{x})=\widehat{W}^{*}(1 \otimes \hat{x}) \widehat{W}, \quad \hat{x} \in L^{\infty}(\widehat{\mathbb{G}}),
$$

where $\widehat{W}=\sigma W^{*} \sigma$, is a co-multiplication on $L^{\infty}(\widehat{\mathbb{G}})$. Furthermore, there exist a left Haar weight $\widehat{\varphi}$ and a right Haar weight $\widehat{\psi}$ on $L^{\infty}(\widehat{\mathbb{G}})$ such that $\widehat{\mathbb{G}}=\left(L^{\infty}(\widehat{\mathbb{G}}), \widehat{\Gamma}, \widehat{\varphi}, \widehat{\psi}\right)$ 
is a locally compact quantum group.

As a consequence, it follows that $W \in L^{\infty}(\mathbb{G}) \bar{\otimes} L^{\infty}(\widehat{\mathbb{G}})$ and $H_{\varphi} \cong H_{\widehat{\varphi}}$. Hence the dual (left) regular representation

$$
\widehat{\lambda}: L^{1}(\widehat{\mathbb{G}}) \ni \hat{f} \mapsto(\hat{f} \otimes \mathrm{id})(\widehat{W})=(\mathrm{id} \otimes \hat{f})\left(W^{*}\right) \in L^{\infty}(\mathbb{G})
$$

generates a von Neumann subalgebra $L^{\infty}(\widehat{\widehat{\mathbb{G}}}):=\left\{\widehat{\lambda}(\hat{f}): \hat{f} \in L^{1}(\widehat{\mathbb{G}})\right\}^{\prime \prime}$ of $L^{\infty}(\mathbb{G})$. In fact, we have a perfect duality.

Theorem 3.3.2 (Quantum Pontrjagin Duality). [106, §1.11] Let $\mathbb{G}$ be a locally compact quantum group. Then

$$
\widehat{\widehat{G}} \cong \mathbb{G}
$$

By considering the right regular representation, we obtain the corresponding dual quantum group $\widehat{\mathbb{G}}^{\prime}=\left(L^{\infty}\left(\widehat{\mathbb{G}}^{\prime}\right), \widehat{\Gamma}^{\prime}, \widehat{\varphi}^{\prime}, \widehat{\psi}^{\prime}\right)$, where the co-multiplication $\widehat{\Gamma}^{\prime}$ is defined by

$$
\widehat{\Gamma}^{\prime}\left(\hat{x}^{\prime}\right)=\widehat{V}^{\prime}\left(\hat{x}^{\prime} \otimes 1\right) \widehat{V}^{\prime *}, \quad \hat{x}^{\prime} \in L^{\infty}\left(\widehat{\mathbb{G}}^{\prime}\right),
$$

and $\widehat{V}^{\prime}=\sigma V^{*} \sigma$. In this case (by considering the duality between $\mathbb{G}$ and $\widehat{\mathbb{G}}^{\prime}$ ), we obtain $\widehat{V}^{\prime} \in L^{\infty}(\mathbb{G}) \bar{\otimes} L^{\infty}\left(\widehat{\mathbb{G}}^{\prime}\right)$ and therefore $V \in L^{\infty}\left(\widehat{\mathbb{G}}^{\prime}\right) \bar{\otimes} L^{\infty}(\mathbb{G})$.

As noted above, the Hilbert spaces in the GNS-constructions of the left Haar weights on $\mathbb{G}$ and $\widehat{\mathbb{G}}$ may be identified. The analogous result holds for the right Haar weights, and it follows that $H_{\varphi} \cong H_{\widehat{\varphi}} \cong H_{\psi} \cong H_{\widehat{\psi}}$. We will therefore denote this common Hilbert space by $L^{2}(\mathbb{G})$. By modular theory, there exists conjugate linear isometries $J, \widehat{J}: L^{2}(\mathbb{G}) \rightarrow L^{2}(\mathbb{G})$ satisfying

$$
J L^{\infty}(\mathbb{G}) J=L^{\infty}(\mathbb{G})^{\prime} \quad \text { and } \quad \widehat{J} L^{\infty}(\widehat{\mathbb{G}}) \widehat{J}=L^{\infty}\left(\widehat{\mathbb{G}}^{\prime}\right) .
$$

Moreover, the unitary $U:=\widehat{J} J$ intertwines the left and right regular representations via $\rho(f)=U \lambda(f) U^{*}, f \in L^{1}(\mathbb{G})$. At the level of the fundamental unitaries, this 
relation becomes

$$
V=\sigma(1 \otimes U) W\left(1 \otimes U^{*}\right) \sigma .
$$

We also record the adjoint formulas $(\widehat{J} \otimes J) W(\widehat{J} \otimes J) W^{*}$ and $(J \otimes \widehat{J}) V(J \otimes \widehat{J})=V^{*}$.

By analogy with the classical setting, we define

$$
C_{0}(\mathbb{G}):={\overline{\widehat{\lambda}}\left(L^{1}(\widehat{\mathbb{G}})\right)}^{\|\cdot\|}
$$

Then $C_{0}(\mathbb{G})$ is a weak* dense $C^{*}$-subalgebra of $L^{\infty}(\mathbb{G})$, called the reduced quantum group associated to $\mathbb{G}$. We define $M(\mathbb{G})$ to be the operator dual $C_{0}(\mathbb{G})^{*}$. Then we have $C_{0}\left(\mathbb{G}_{a}\right)=C_{0}(G)$ and $M\left(\mathbb{G}_{a}\right)=M(G)$.

Example 3.3.3. If $G$ is a locally compact group, $\mathbb{G}_{a}=\left(L^{\infty}(G), \Gamma_{a}, \varphi_{a}, \psi_{a}\right)$ and $\mathbb{G}_{s}=$ $\left(\mathcal{L}(G), \Gamma_{s}, \varphi_{s}\right)$ are dual objects in the category of locally compact quantum groups, therefore establishing Pontrjagin duality for non-abelian locally compact groups.

An important consequence of this duality is given by the following proposition, known as Heisenberg's theorem.

Proposition 3.3.4. Let $G$ be a locally compact group. Then

$$
L^{\infty}(G) \cap \mathcal{L}(G)=L^{\infty}(G) \cap \mathcal{R}(G)=\mathbb{C} 1 .
$$

Equivalently, $L^{\infty}(G) \vee \mathcal{L}(G)=L^{\infty}(G) \vee \mathcal{R}(G)=\mathcal{B}\left(L^{2}(G)\right)$, where $L^{\infty}(G) \vee \mathcal{L}(G)$ denotes the von Neumann algebra generated by $L^{\infty}(G)$ and $\mathcal{L}(G)$.

The following two propositions constitute the generalization of Heisenberg's theorem to the non-commutative setting. Here, the notation ${\overline{\left\langle L^{\infty}(\mathbb{G}) L^{\infty}(\widehat{\mathbb{G}})\right\rangle}}^{w *}$ refers to the weak* closed linear span of $L^{\infty}(\mathbb{G}) L^{\infty}(\widehat{\mathbb{G}})=\left\{x \hat{x}: x \in L^{\infty}(\mathbb{G}), \hat{x} \in L^{\infty}(\widehat{\mathbb{G}})\right\}$. 
Proposition 3.3.5. [112, Proposition 3.4] Let $\mathbb{G}$ be a locally compact quantum group. Then

$$
\begin{gathered}
L^{\infty}(\mathbb{G}) \cap L^{\infty}(\widehat{\mathbb{G}})=L^{\infty}(\mathbb{G})^{\prime} \cap L^{\infty}(\widehat{\mathbb{G}})= \\
=L^{\infty}(\mathbb{G}) \cap L^{\infty}\left(\widehat{\mathbb{G}}^{\prime}\right)=L^{\infty}(\mathbb{G})^{\prime} \cap L^{\infty}\left(\widehat{\mathbb{G}}^{\prime}\right)=\mathbb{C} 1 .
\end{gathered}
$$

Proposition 3.3.6. [110, Proposition 2.5] Let $\mathbb{G}$ be a locally compact quantum group. Then

$$
\begin{aligned}
& {\overline{\left\langle L^{\infty}\left(\widehat{\mathbb{G}}^{\prime}\right) L^{\infty}(\widehat{\mathbb{G}})\right\rangle}}^{w *}={\overline{\left\langle L^{\infty}(\widehat{\mathbb{G}}) L^{\infty}(\mathbb{G})^{\prime}\right\rangle}}^{w *}= \\
= & {\overline{\left\langle L^{\infty}(\mathbb{G}) L^{\infty}(\widehat{\mathbb{G}})\right\rangle}}^{w *}={\overline{\left\langle L^{\infty}(\mathbb{G})^{\prime} L^{\infty}\left(\widehat{\mathbb{G}}^{\prime}\right)\right\rangle}}^{w *}=\mathcal{B}\left(L^{2}(\mathbb{G})\right) .
\end{aligned}
$$

\subsection{The Antipode}

The antipode is the quantum analogue of group inversion. By way of motivation, let us consider the commutative case.

If $G$ is a locally compact group, define $S: L^{\infty}(G) \rightarrow L^{\infty}(G)$ by $S(f)(s)=f\left(s^{-1}\right)$, $s \in G$. We may also define a corresponding right Haar weight $\psi$ on $L^{\infty}(G)$ by

$$
\psi(f)=\int_{G} f\left(s^{-1}\right) d s, \quad f \in L^{\infty}(G),
$$

where $d s$ denotes a fixed left Haar measure on $G$. In terms of the co-multiplication on $L^{\infty}(G)$, it follows that

$$
S\left((\psi \otimes \mathrm{id})\left((f \otimes 1) \Gamma_{a}(g)\right)\right)=(\psi \otimes \mathrm{id})\left(\Gamma_{a}(f)(g \otimes 1)\right), \quad f, g \in L^{\infty}(G) .
$$


Indeed,

$$
\begin{aligned}
S\left((\psi \otimes \mathrm{id})\left((f \otimes 1) \Gamma_{a}(g)\right)\right)(s) & =(\psi \otimes \mathrm{id})\left((f \otimes 1) \Gamma_{a}(g)\right)\left(s^{-1}\right) \\
& =\int_{G}(f \otimes 1) \Gamma_{a}(g)\left(t^{-1}, s^{-1}\right) d t \\
& =\int_{G} f\left(t^{-1}\right) g\left(t^{-1} s^{-1}\right) d t \\
& =\int_{G} f\left(t^{-1} s\right) g\left(t^{-1}\right) d t \\
& =(\psi \otimes \mathrm{id})\left(\Gamma_{a}(f)(g \otimes 1)\right)(s) .
\end{aligned}
$$

In order to obtain a similar formula for general $\mathbb{G}$, we begin at the level of the Hilbert space $L^{2}(\mathbb{G})$ on which it acts.

Proposition 3.4.1. [106, Proposition 1.14.3] For every $x, y \in \mathcal{N}_{\varphi}^{*} \mathcal{N}_{\psi}$, we have $(\psi \otimes \mathrm{id})\left(\Gamma\left(x^{*}\right)(y \otimes 1)\right) \in \mathcal{N}_{\varphi}$. If we define

$$
G \Lambda_{\varphi}\left((\psi \otimes \mathrm{id})\left(\Gamma\left(x^{*}\right)(y \otimes 1)\right)\right)=\Lambda_{\varphi}\left((\psi \otimes \mathrm{id})\left(\Gamma\left(y^{*}\right)(x \otimes 1)\right)\right)
$$

then $G$ is a closed densely defined anti-linear operator on $L^{2}(\mathbb{G})$. Furthermore, if $I N^{\frac{1}{2}}$ is its polar decomposition, then

1. $I=I^{*}$;

2. $I^{2}=1$;

3. $I N I=N^{-1}$.

Defining the operators

$$
\begin{aligned}
& R: L^{\infty}(\mathbb{G}) \ni x \mapsto I x^{*} I \in L^{\infty}(\mathbb{G}) ; \\
& \tau_{t}: L^{\infty}(\mathbb{G}) \ni x \mapsto N^{-i t} x N^{i t} \in L^{\infty}(\mathbb{G}), \quad t \in \mathbb{R},
\end{aligned}
$$

it follows that $R$ is a ${ }^{*}$-anti-automorphism on $L^{\infty}(\mathbb{G})$ such that $R^{2}=1$, and $\left(\tau_{t}\right)_{t \in \mathbb{R}}$ is a strongly continuous one-parameter group of automorphisms on $L^{\infty}(\mathbb{G})$. We call 
$R$ the unitary antipode of $\mathbb{G}$, and $\left(\tau_{t}\right)_{t \in \mathbb{R}}$ the scaling group of $\mathbb{G}$. Note that $\tau_{t} R=R \tau_{t}$ for all $t \in \mathbb{R}$ since $I N I=N^{-1}$. It follows that $R(x)=\widehat{J} x^{*} \widehat{J}$, for $x \in L^{\infty}(\mathbb{G})[64$, Proposition 2.1].

Definition 3.4.2. The antipode of $\mathbb{G}$ is defined as $S=R \tau_{-\frac{i}{2}}$, where $\tau_{-\frac{i}{2}}$ is the analytic extension of $\left(\tau_{t}\right)_{t \in \mathbb{R}}$ at $z=-\frac{i}{2} \in \mathbb{C}$. We let $\mathcal{D}(S)$ denote its domain.

Theorem 3.4.3. [106, §1.4] The antipode $S$ has the following properties:

1. $S$ is densely defined and has dense range;

2. $S$ is injective and $S^{-1}=R \tau_{\frac{i}{2}}=\tau_{\frac{i}{2}} R$;

3. $S$ is anti-multiplicative, that is, for all $x, y \in \mathcal{D}(S), x y \in \mathcal{D}(S)$ and $S(x y)=$ $S(y) S(x)$;

4. for all $x \in \mathcal{D}(S), S(x)^{*} \in \mathcal{D}(S)$ and $S\left(S(x)^{*}\right)^{*}=x$;

5. for every $x, y \in \mathcal{N}_{\psi}$, we have $(\psi \otimes \mathrm{id})\left(\left(x^{*} \otimes 1\right) \Gamma(y)\right) \in \mathcal{D}(S)$ and

$$
S\left((\psi \otimes \mathrm{id})\left(\left(x^{*} \otimes 1\right) \Gamma(y)\right)\right)=(\psi \otimes \mathrm{id})\left(\Gamma\left(x^{*}\right)(y \otimes 1)\right)
$$

6. $\mathcal{D}(S)$ contains $(\mathrm{id} \otimes \hat{f})(W)$ for all $\hat{f} \in L^{1}(\widehat{\mathbb{G}})$, and

$$
S((\mathrm{id} \otimes \hat{f})(W))=(\mathrm{id} \otimes \hat{f})\left(W^{*}\right) .
$$

Let $\mathbb{G}$ be a locally compact quantum group such that the antipode $S$ is bounded. Then the scaling group is trivial and $S=R$, the unitary antipode. If, in addition, the left Haar weight $\varphi$ satisfies

$$
(\mathrm{id} \otimes \varphi)\left(\left(1 \otimes y^{*}\right) \Gamma(x)\right)=R\left((\mathrm{id} \otimes \varphi)\left(\Gamma\left(y^{*}\right)(1 \otimes x)\right)\right), \quad x, y \in \mathcal{N}_{\varphi}
$$


and $R \circ \sigma_{t}^{\varphi}=\sigma_{-t}^{\varphi} \circ R$ for all $t \in \mathbb{R}$, where $\sigma_{t}^{\varphi}$ is the modular automorphism group of $\varphi$, then we say that $\mathbb{G}$ is Kac algebra. Historically, these were the first examples of locally compact quantum groups. Indeed, all commutative and co-commutative quantum groups are Kac algebras. The reader is referred to [30] for details.

\subsection{Quantum Groups in the Universal Setting}

In the classical setting one uses the antipode $S$ to define an involution on $L^{1}(G)=$ $L^{1}\left(\widehat{\mathbb{G}_{s}}\right)$, whose enveloping $C^{*}$-algebra $C^{*}(G)$ plays an important role in abstract harmonic analysis. For general locally compact quantum groups, one would like an analogue of the group $C^{*}$-algebra. Unfortunately, the straightforward generalization to define an involution on $L^{1}(\widehat{\mathbb{G}})$ respected by the left regular representation is not possible as the antipode $S$ may be unbounded. Instead, one defines an involution on a dense subalgebra of $L^{1}(\widehat{\mathbb{G}})$, then passes to its enveloping $C^{*}$-algebra in the same manner as in the classical case. The resulting object is known as the universal quantum group associated to $\mathbb{G}$, and we outline its construction below; for details, see [61].

Let $\mathbb{G}$ be a locally compact quantum group with dual $\widehat{\mathbb{G}}$, and let $L_{*}^{1}(\widehat{\mathbb{G}})$ be the subspace of $L^{1}(\widehat{\mathbb{G}})$ defined by

$$
L_{*}^{1}(\widehat{\mathbb{G}})=\left\{\hat{f} \in L^{1}(\widehat{\mathbb{G}}): \exists \hat{g} \in L^{1}(\widehat{\mathbb{G}}) \quad \text { s.t. } \quad \hat{g}(\hat{x})=\hat{f}^{*} \circ \widehat{S}(\hat{x}) \quad \forall \hat{x} \in \mathcal{D}(\widehat{S})\right\}
$$

where $\hat{f}^{*}(\hat{x})=\overline{\hat{f}\left(\hat{x}^{*}\right)}, \hat{x} \in L^{\infty}(\widehat{\mathbb{G}})$. It is known from [106, $\left.\S 1.13\right]$ that $L_{*}^{1}(\widehat{\mathbb{G}})$ is a dense subalgebra of $L^{1}(\widehat{\mathbb{G}})$. There is an involution on $L_{*}^{1}(\widehat{\mathbb{G}})$ given by $\hat{f}^{o}=\hat{f}^{*} \circ \widehat{S}$, such that $L_{*}^{1}(\widehat{\mathbb{G}})$ becomes a Banach *-algebra under the norm

$$
\|\hat{f}\|_{*}=\max \left\{\|\hat{f}\|,\left\|\hat{f}^{o}\right\|\right\} .
$$

Moreover, the restricted left regular representation $\widehat{\lambda}: L_{*}^{1}(\widehat{\mathbb{G}}) \rightarrow C_{0}(\mathbb{G})$ becomes a 
*-homomorphism. Indeed, by Theorem 3.4.3, the domain of $\widehat{S}$ contains (id $\otimes f)(\widehat{W})$, $f \in L^{1}(\mathbb{G})$, and since $\widehat{S}((\mathrm{id} \otimes f)(\widehat{W}))=(\mathrm{id} \otimes f)\left(\widehat{W}^{*}\right)$, we have

$$
\begin{aligned}
\left\langle\widehat{\lambda}\left(\hat{f}^{o}\right), f\right\rangle & =\left\langle\left(\hat{f}^{o} \otimes \mathrm{id}\right)(\widehat{W}), f\right\rangle \\
& =\left\langle\hat{f}^{o},(\mathrm{id} \otimes f)(\widehat{W})\right\rangle \\
& =\left\langle\hat{f}^{*},(\mathrm{id} \otimes f)\left(\widehat{W}^{*}\right)\right\rangle \\
& =\left\langle\left(\hat{f}^{*} \otimes \mathrm{id}\right)\left(\widehat{W}^{*}\right), f\right\rangle \\
& =\left\langle[(\hat{f} \otimes \mathrm{id})(\widehat{W})]^{*}, f\right\rangle \\
& =\left\langle\widehat{\lambda}(\hat{f})^{*}, f\right\rangle,
\end{aligned}
$$

where in the second last step we have used the normality of the linear functional $\left(\hat{f}^{*} \otimes \mathrm{id}\right): L^{\infty}(\widehat{\mathbb{G}}) \bar{\otimes} L^{\infty}(\mathbb{G}) \rightarrow L^{\infty}(\mathbb{G})$, together with the weak* continuity of the adjoint.

Next, define the enveloping $C^{*}$-algebra by introducing the following norm for all $\hat{f} \in L_{*}^{1}(\widehat{\mathbb{G}})$,

$$
\|\hat{f}\|_{u}=\sup \left\{\|\pi(\hat{f})\| \mid \pi: L_{*}^{1}(\widehat{\mathbb{G}}) \rightarrow \mathcal{B}(H) \text { is a s.nd. *-representation }\right\} .
$$

Taking the completion, we obtain the universal quantum group $C^{*}$-algebra of $\mathbb{G}$, denoted $C_{u}(\mathbb{G})$. Writing $\widehat{\lambda}_{u}$ for the embedding of $L_{*}^{1}(\widehat{\mathbb{G}})$ into $C_{u}(\mathbb{G})$, the pair $\left(C_{u}(\mathbb{G}), \widehat{\lambda}_{u}\right)$ is determined (up to ${ }^{*}$-isomorphism) by the following universal property: if $\mathcal{A}$ is a $C^{*}$-algebra and $\pi: L_{*}^{1}(\widehat{\mathbb{G}}) \rightarrow \mathcal{A}$ is a ${ }^{*}$-representation, then there exists a unique *-representation $\pi_{u}: C_{u}(\mathbb{G}) \rightarrow \mathcal{A}$ such that $\pi_{u} \circ \widehat{\lambda}_{u}=\pi$.

For commutative quantum groups $\mathbb{G}_{a}$, we have $C_{u}\left(\mathbb{G}_{a}\right)=C_{0}(G)$ and for cocommutative quantum groups $\mathbb{G}_{s}$, we obtain $C_{u}\left(\mathbb{G}_{s}\right)=C^{*}(G)$, the full group $C^{*}$ algebra of $G$, thereby making $C_{u}(\mathbb{G})$ the appropriate generalization to the quantum group setting. 
Definition 3.5.1. Let $\mathcal{A}$ be a $C^{*}$-algebra. The idealizer of $\mathcal{A}$ in $\left(\mathcal{A}^{* *}, \square\right)$, denoted $M(\mathcal{A})$, is defined by

$$
\left\{x \in \mathcal{A}^{* *}: x \square a, a \square x \in \mathcal{A} \text { for all } a \in \mathcal{A}\right\},
$$

and is called the multiplier algebra of $\mathcal{A}$.

Note that $M(\mathcal{A})$ is a $C^{*}$-subalgebra of $\mathcal{A}^{* *}$, containing $\mathcal{A}$ as a norm-closed two sided ideal, and $\mathcal{A}$ is unital if and only if $M(\mathcal{A})=\mathcal{A}$. Moreover, if $\mathcal{A}$ is a $C^{*}$ subalgebra of $\mathcal{B}(H)$ for a Hilbert space $H$, then $M(\mathcal{A}) \subseteq \mathcal{B}(H)$ [86, Proposition 2.12.9]. For instance, if $\mathcal{A}=\mathcal{K}(H)$, the $C^{*}$-algebra of compact operators $H$, then $M(\mathcal{A})=\mathcal{B}(H)$. For details on multiplier algebras, see [86, §2.12].

Definition 3.5.2. Let $\mathcal{A}$ and $\mathcal{B}$ be $C^{*}$-algebras. For every $a \in \mathcal{A}$, define a semi-norm on $M(\mathcal{A})$ by

$$
\|x\|_{a}=\|x \square a\|+\|a \square x\|, \quad x \in M(\mathcal{A}) .
$$

The weak topology generated by the semi-norms $\left\{\|\cdot\|_{a}: a \in \mathcal{A}\right\}$ is called the strict topology in $M(\mathcal{A})$. We say that a linear map $\pi: \mathcal{A} \rightarrow M(\mathcal{B})$ is strict if it is norm bounded and strictly continuous on bounded sets.

One can show that if $\pi: \mathcal{A} \rightarrow M(\mathcal{B})$ is strict, then it has a unique bounded linear extension $\tilde{\pi}: M(\mathcal{A}) \rightarrow M(\mathcal{B})$ which is strictly continuous on bounded sets $[62, \S 7]$. Three examples of importance are bounded linear functionals $\varphi \in \mathcal{A}^{*}$, their associated slice maps

$$
\varphi \otimes \text { id }: \mathcal{A} \otimes_{\min } \mathcal{B} \rightarrow M(\mathcal{B}) \quad \text { and } \quad \text { id } \otimes \varphi: \mathcal{B} \otimes_{\min } \mathcal{A} \rightarrow M(\mathcal{B}),
$$

and nondegenerate ${ }^{*}$-representations $\pi: \mathcal{A} \rightarrow M(\mathcal{B})$, meaning

$$
\mathcal{B}=\overline{\{\pi(a) \square b: a \in \mathcal{A}, \quad b \in \mathcal{B}\}}^{\|\cdot\|} .
$$


In what follows, we will refrain from writing $\widetilde{\pi}$ for the extensions of strict linear mappings, and simply write $\pi$.

The multiplier algebra of $C_{0}(\mathbb{G})$ plays a prominent role in the $C^{*}$-algebraic formulation of locally compact quantum groups. For instance, $\left.\Gamma\right|_{C_{0}(\mathbb{G})}: C_{0}(\mathbb{G}) \rightarrow$ $M\left(C_{0}(\mathbb{G}) \otimes_{\min } C_{0}(\mathbb{G})\right)$ is the co-multiplication on the reduced quantum group $C_{0}(\mathbb{G})$ [4]. This allows one to construct a multiplication on $M(\mathbb{G})$ via

$$
\langle\mu \star \nu, x\rangle=\langle\mu \otimes \nu, \Gamma(x)\rangle, \quad x \in C_{0}(\mathbb{G}), \mu, \nu \in M(\mathbb{G}),
$$

where $\mu \otimes \nu \in M\left(C_{0}(\mathbb{G}) \otimes_{\min } C_{0}(\mathbb{G})\right)^{*}$ is understood as the composition $\mu \circ(\mathrm{id} \otimes \nu$ ). Under $\star$, the space $M(\mathbb{G})$ becomes a completely contractive Banach algebra containing $L^{1}(\mathbb{G})$ as a norm closed two-sided ideal via the map $\left.L^{\infty}(\mathbb{G})^{*} \supseteq L^{1}(\mathbb{G}) \ni f \mapsto f\right|_{C_{0}(\mathbb{G})} \in$ $M(\mathbb{G})$.

Since $\hat{\lambda}: L_{*}^{1}(\widehat{\mathbb{G}}) \rightarrow C_{0}(\mathbb{G})$ is a ${ }^{*}$-homomorphism, by the universal property of $C_{u}(\mathbb{G})$, there exists a unique surjective ${ }^{*}$-homomorphism $\pi: C_{u}(\mathbb{G}) \rightarrow C_{0}(\mathbb{G})$ such that $\pi \circ \widehat{\lambda}_{u}(\hat{f})=\hat{\lambda}(\hat{f})$ for all $\hat{f} \in L_{*}^{1}(\widehat{\mathbb{G}})$. Furthermore, there is a non-degenerate co-associative ${ }^{*}$-homomorphism $\Gamma_{u}: C_{u}(\mathbb{G}) \rightarrow M\left(C_{u}(\mathbb{G}) \otimes_{\min } C_{u}(\mathbb{G})\right)$ satisfying

$$
(\pi \otimes \pi) \circ \Gamma_{u}=\Gamma \circ \pi
$$

see [61, Proposition 6.2]. The space $C_{u}(\mathbb{G})^{*}$ then has the structure of a unital completely contractive Banach algebra via

$$
\left\langle\mu \star_{u} \nu, x\right\rangle=\left\langle\mu \otimes \nu, \Gamma_{u}(x)\right\rangle, \quad x \in C_{u}(\mathbb{G}), \mu, \nu \in C_{u}(\mathbb{G})^{*} .
$$

Under this multiplication, the map $i: L^{1}(\mathbb{G}) \rightarrow C_{u}(\mathbb{G})^{*}$ given by the composition of the inclusion $L^{1}(\mathbb{G}) \subseteq M(\mathbb{G})$ and $\pi^{*}: M(\mathbb{G}) \rightarrow C_{u}(\mathbb{G})^{*}$ is a completely isometric homomorphism. It follows that $i\left(L^{1}(\mathbb{G})\right)$ is a norm closed two-sided ideal in $C_{u}(\mathbb{G})^{*}$ [61, Proposition 8.3]. 


\subsection{Compact Quantum Groups}

Recall that a locally compact quantum group $\mathbb{G}=\left(L^{\infty}(\mathbb{G}), \Gamma, \varphi, \psi\right)$ is compact if $\varphi$ is finite. In this case, $\varphi$ is both left and right invariant, and we normalize by $\varphi(1)=1$.

A unitary co-representation of $\mathbb{G}$ is a unitary $U \in L^{\infty}(\mathbb{G}) \bar{\otimes} \mathcal{B}(H)$ which satisfies $(\Gamma \otimes \mathrm{id})(U)=U_{13} U_{23}$. The co-representation $U$ then defines a homomorphism $\pi_{U}$ : $L^{1}(\mathbb{G}) \rightarrow \mathcal{B}(H)$ via

$$
\pi_{U}(f)=(f \otimes \mathrm{id})(U), \quad f \in L^{1}(\mathbb{G}) .
$$

We say that $U$ is irreducible if the corresponding representation $\pi_{U}$ is irreducible.

We have the following facts about $\mathbb{G}$ : every irreducible co-representation $u^{\alpha}$ is finite-dimensional and is unitarily equivalent to a sub-representation of $W$, and every unitary co-representation of $\mathbb{G}$ can be decomposed into a direct sum of irreducible representations. We let $\operatorname{Irr}(\mathbb{G}):=\left\{u^{\alpha}\right\}$ denote a complete set of representatives of irreducible co-representations of $\mathbb{G}$ which are pairwise in-equivalent (cf. [118]). Slicing by vector functionals $\omega_{i j}=\omega_{e_{j}, e_{i}}$ relative to an orthonormal basis of $H_{\alpha}$, we obtain elements $u_{i j}^{\alpha}=\left(\right.$ id $\left.\otimes \omega_{i j}\right)\left(u^{\alpha}\right) \in L^{\infty}(\mathbb{G})$ satisfying

$$
\Gamma\left(u_{i j}^{\alpha}\right)=\sum_{k=1}^{n_{\alpha}} u_{i k}^{\alpha} \otimes u_{k j}^{\alpha}
$$

for all $1 \leq i, j \leq n_{\alpha}$. The linear space $\mathcal{A}:=\operatorname{span}\left\{u_{i j}^{\alpha} \mid \alpha \in \operatorname{Irr}(\mathbb{G}), 1 \leq i, j \leq n_{\alpha}\right\}$ forms unital Hopf *-algebra which is linearly dense in $C(\mathbb{G}):=C_{0}(\mathbb{G})$ [118]. On $\mathcal{A}$, the antipode and Haar weight are given respectively by

$$
S\left(u_{i j}^{\alpha}\right)=\left(u_{j i}^{\alpha}\right)^{*} \quad \text { and } \quad \varphi\left(u_{i j}^{\alpha}\right)=\delta_{\alpha, \alpha_{0}}, \quad 1 \leq i, j \leq n_{\alpha}, \alpha \in \operatorname{Irr}(\mathbb{G}),
$$

where $\alpha_{0}$ is the trivial representation, satisfying $u^{\alpha_{0}}=1$.

In this case, the dual quantum group $\widehat{\mathbb{G}}$ is discrete and has underlying von Neu- 
mann algebra

$$
\ell^{\infty}(\widehat{\mathbb{G}})=\bigoplus_{\alpha \in \operatorname{Irr}(\mathbb{G})} M_{n_{\alpha}}(\mathbb{C}) .
$$

For every $\alpha \in \operatorname{Irr}(\mathbb{G})$ there exists a positive invertible matrix $F^{\alpha} \in M_{n_{\alpha}}(\mathbb{C})$ such that the corresponding " $F$-matrices" implement the left Haar weight of the dual $\widehat{\mathbb{G}}$ in the sense that

$$
\widehat{\varphi}(x)=\sum_{\alpha \in \operatorname{Irr}(\mathbb{G})} \operatorname{tr}\left(F^{\alpha}\right) \operatorname{tr}\left(F^{\alpha} x\right), \quad x \in \mathcal{M}_{\widehat{\varphi}} .
$$

Without loss of generality, we may assume that $F^{\alpha}=\operatorname{diag}\left(\lambda_{1}^{\alpha}, \cdots, \lambda_{n_{\alpha}}^{\alpha}\right)[19$, Proposition 2.1]. Since $\operatorname{tr}\left(F^{\alpha}\right)=\operatorname{tr}\left(F^{\alpha}\right)^{-1}$, it follows that

$$
\sum_{i=1}^{n_{\alpha}} \lambda_{i}^{\alpha}=\sum_{i=1}^{n_{\alpha}} \frac{1}{\lambda_{i}^{\alpha}}=\operatorname{tr}\left(F^{\alpha}\right)=: d_{\alpha}
$$

where $d_{\alpha}$ is the quantum dimension of $u^{\alpha}$. If $\mathbb{G}$ is a Kac algebra, then $d_{\alpha}=n_{\alpha}$ and $F^{\alpha}=1_{n_{\alpha}}$ for all $\alpha \in \operatorname{Irr}(\mathbb{G})$.

For a concrete example of a compact quantum group, we present an instance of the Van Daele-Wang construction of universal quantum groups [111].

Example 3.6.1. Let $N \geq 2$ be an integer and let $F \in G L(N, \mathbb{C})$ be such that $F \bar{F}=\lambda I_{N}$ for some $\lambda \in \mathbb{R}$. We let $A_{o}(F)$ be the universal $C^{*}$-algebra generated by $N^{2}$ elements $u_{i j}$ such that the matrix $u=\left[u_{i j}\right]$ is unitary and $F \bar{u} F^{-1}=u$, where $\bar{u}=\left[u_{i j}^{*}\right]$. Define $\Gamma_{u}: A_{o}(F) \rightarrow A_{o}(F) \otimes_{\min } A_{o}(F)$ on the generators by

$$
\Gamma_{u}\left(u_{i j}\right)=\sum_{k=1}^{N} u_{i k} \otimes u_{k j}, \quad 1 \leq i, j \leq N .
$$

It follows that there exists a unique Haar state $\varphi$ on $A_{o}(F)$ satisfying $(\operatorname{id} \otimes \varphi) \Gamma_{u}(x)=$ $(\varphi \otimes \mathrm{id}) \Gamma_{u}(x)=\varphi(x) 1, x \in A_{o}(F)$ [118]. The GNS construction $\left(\pi_{\varphi}, \xi_{\varphi}\right)$ of $\varphi$ then yields a von Neumann algebra $L^{\infty}\left(\mathbb{G}_{F}\right):=\pi_{\varphi}\left(A_{o}(F)\right)^{\prime \prime} \subseteq \mathcal{B}\left(H_{\varphi}\right)$ and a comultiplication $\Gamma: L^{\infty}\left(\mathbb{G}_{F}\right) \rightarrow L^{\infty}\left(\mathbb{G}_{F}\right) \bar{\otimes} L^{\infty}\left(\mathbb{G}_{F}\right)$ such that $\mathbb{G}_{F}=\left(L^{\infty}\left(\mathbb{G}_{F}\right), \Gamma, \omega_{\xi_{\varphi}}\right)$ becomes a compact quantum group, called the free orthogonal quantum group (with 
parameter matrix $F)$. In this example, $C_{u}\left(\mathbb{G}_{F}\right)=A_{o}(F)$ and $C_{0}\left(\mathbb{G}_{F}\right)=C\left(\mathbb{G}_{F}\right)=$ $\pi_{\varphi}\left(A_{o}(F)\right) \subseteq \mathcal{B}\left(H_{\varphi}\right)$. We mention a few special cases of interest:

- It is known that $\mathbb{G}_{F}$ is co-amenable if and only if $N=2$ [5, Corollaire 1], and hence, $\widehat{\mathbb{G}_{F}}$ is amenable if and only if $N=2$ by Tomatsu's theorem [104, Theorem 3.8].

- When $F=\left(\begin{array}{cc}0 & 1 \\ -q^{-1} & 0\end{array}\right)$, for $q \in[-1,1], q \neq 0$, we recover Woronowicz's deformation $S U_{q}(2)$ of the compact Lie group $S U(2)$ [117], namely as the universal $C^{*}$-algebra generated by $\alpha$ and $\gamma$ such that the matrix

$$
\left(\begin{array}{cc}
\alpha & -q \gamma^{*} \\
\gamma & \alpha^{*}
\end{array}\right)
$$

is unitary.

- It is known that $\mathbb{G}_{F}$ is a Kac algebra precisely when $F$ is a scalar multiple of a unitary matrix (see [5, Théorème 3]).

- When $F=I_{N}$, and we take the universal commutative $C^{*}$-algebra generated by $u_{i j}$ satisfying the above relations, then we obtain $C(O(N))$, the commutative $C^{*}$-algebra of continuous functions on the orthogonal group $O(N)$.

\subsection{Completely Bounded Multipliers}

Building on work of Cowling, de Cannière, Haagerup and Kraus regarding completely bounded multipliers of the Fourier algebra [16, 24, 43], Kraus and Ruan introduced the notion of completely bounded multipliers for Kac algebras [59], which was then extended to the setting of locally compact quantum groups in $[53,49]$. We finish this chapter with an overview of the relevant theory that will be important in the sequel. 
Definition 3.7.1. Let $\mathbb{G}$ be a locally compact quantum group. An element $\hat{b}^{\prime} \in$ $L^{\infty}(\widehat{\mathbb{G}})^{\prime}$ is said to be a completely bounded right multiplier of $L^{1}(\mathbb{G})$ if $\rho(f) \hat{b}^{\prime} \in$ $\rho\left(L^{1}(\mathbb{G})\right)$ for all $f \in L^{1}(\mathbb{G})$ and the induced map

$$
m_{\hat{b}^{\prime}}^{r}: L^{1}(\mathbb{G}) \ni f \mapsto \rho^{-1}\left(\rho(f) \hat{b}^{\prime}\right) \in L^{1}(\mathbb{G})
$$

is completely bounded on $L^{1}(\mathbb{G})$. We let $M_{c b}^{r}\left(L^{1}(\mathbb{G})\right)$ denote the space of all completely bounded right multipliers of $L^{1}(\mathbb{G})$. It is easy to see that $M_{c b}^{r}\left(L^{1}(\mathbb{G})\right)$ is a completely contractive Banach algebra with respect to the norm

$$
\left\|\left[\hat{b}_{i j}^{\prime}\right]\right\|_{M_{n}\left(M_{c b}^{r}\left(L^{1}(\mathbb{G})\right)\right)}=\left\|\left[m_{\hat{b}_{i j}}^{r}\right]\right\|_{c b} .
$$

Completely bounded left multipliers are defined analogously as those $\hat{b} \in L^{\infty}(\widehat{\mathbb{G}})$ such that $\hat{b} \lambda\left(L^{1}(\mathbb{G})\right) \subseteq \lambda\left(L^{1}(\mathbb{G})\right)$ and the induced map

$$
m_{\hat{b}}^{l}: L^{1}(\mathbb{G}) \ni f \mapsto \lambda^{-1}(\hat{b} \lambda(f)) \in L^{1}(\mathbb{G})
$$

is completely bounded. We let $M_{c b}^{l}\left(L^{1}(\mathbb{G})\right)$ denote the completely contractive Banach algebra of completely bounded left multipliers.

Given $\hat{b}^{\prime} \in M_{c b}^{r}\left(L^{1}(\mathbb{G})\right)$, the adjoint $\Theta^{r}\left(\hat{b}^{\prime}\right):=\left(m_{\hat{b}^{\prime}}^{r}\right)^{*}$ defines a normal completely bounded right $L^{1}(\mathbb{G})$-module map on $L^{\infty}(\mathbb{G})$. When $\hat{b}^{\prime}=\rho(f)$, the map $\Theta^{r}(\rho(f))$ is nothing but the convolution action of $L^{1}(\mathbb{G})$ on $L^{\infty}(\mathbb{G})$, that is,

$$
\Theta^{r}(\rho(f))(x)=f \star x=(\mathrm{id} \otimes f) \Gamma(x), \quad x \in L^{\infty}(\mathbb{G}) .
$$

In general, the restriction $\left.\Theta^{r}\left(\hat{b}^{\prime}\right)\right|_{C_{0}(\mathbb{G})}$ leaves $C_{0}(\mathbb{G})$ invariant by [53, Proposition 4.1], and, together with [53, Proposition 4.2], we have the completely isometric identifications

$$
M_{c b}^{r}\left(L^{1}(\mathbb{G})\right) \cong \mathcal{C B}_{L^{1}(\mathbb{G})}^{\sigma}\left(L^{\infty}(\mathbb{G})\right) \cong \mathcal{C B}_{L^{1}(\mathbb{G})}\left(C_{0}(\mathbb{G})\right)
$$


In the commutative case, $M_{c b}^{r}\left(L^{1}\left(\mathbb{G}_{a}\right)\right) \cong M(G)$ by Wendel's theorem [113], and the action on $L^{\infty}(G)$ is given by

$$
\Theta^{r}(\mu)(f)(s)=\mu \star f(s)=\int_{G} f(s t) d \mu(t), \quad \mu \in M(G), f \in L^{\infty}(G) .
$$

In the co-commutative case, $M_{c b}^{r}\left(L^{1}\left(\mathbb{G}_{s}\right)\right)=M_{c b} A(G)$, the completely bounded multipliers of the Fourier algebra, and the action on $\mathcal{L}(G)$ is given by

$$
\widehat{\Theta}^{r}(\varphi)(\lambda(s))=\varphi(s) \lambda(s), \quad \varphi \in M_{c b} A(G), s \in G .
$$

For a locally compact quantum group $\mathbb{G}$, it is known that $M_{c b}^{r}\left(L^{1}(\mathbb{G})\right)$ is a dual space [49, Theorem 3.5], with predual $Q_{c b}^{r}\left(L^{1}(\mathbb{G})\right)$ given as the completion of $L^{1}\left(\widehat{\mathbb{G}}^{\prime}\right)$ under the completely contractive embedding

$$
L^{1}\left(\widehat{\mathbb{G}}^{\prime}\right) \ni \widehat{f}^{\prime} \mapsto \alpha_{\hat{f}^{\prime}} \in M_{c b}^{r}\left(L^{1}(\mathbb{G})\right)^{*},
$$

where $\left\langle\alpha_{\hat{f}^{\prime}}, \hat{b}^{\prime}\right\rangle=\left\langle\hat{b}^{\prime}, \hat{f}^{\prime}\right\rangle$, for $\hat{f}^{\prime} \in L^{1}\left(\widehat{\mathbb{G}}^{\prime}\right)$ and $\hat{b}^{\prime} \in M_{c b}^{r}\left(L^{1}(\mathbb{G})\right)$. In the co-commutative setting, Haagerup and Kraus gave a representation for elements of $Q_{c b} A(G)$ as $\Omega_{X, \rho}$ for $X \in C_{\lambda}^{*}(G) \otimes_{\min } K_{\infty}$ and $\rho \in A(G) \widehat{\otimes} T_{\infty}$ [43, Proposition 1.5], where

$$
\left\langle\varphi, \Omega_{X, \rho}\right\rangle=\left\langle\left(\widehat{\Theta}^{r}(\varphi) \otimes \operatorname{id}_{K_{\infty}}\right)(X), \rho\right\rangle, \quad \varphi \in M_{c b} A(G) .
$$

This was later generalized to the setting of Kac algbras by Kraus and Ruan [60, Theorem 3.3]. Relying upon the general result [43, Lemma 1.6], their argument readily extends to arbitrary locally compact quantum groups.

Proposition 3.7.2. Let $\mathbb{G}$ be a locally compact quantum group. Then

$$
Q_{c b}^{r}\left(L^{1}(\mathbb{G})\right)=\left\{\Omega_{X, \rho} \mid X \in C_{0}(\mathbb{G}) \otimes_{\min } K_{\infty}, \rho \in L^{1}(\mathbb{G}) \widehat{\otimes} T_{\infty}\right\}
$$

where $\left\langle\hat{b}, \Omega_{X, \rho}\right\rangle=\left\langle\left(\Theta^{r}\left(\hat{b}^{\prime}\right) \otimes \operatorname{id}_{K_{\infty}}\right)(X), \rho\right\rangle, \hat{b}^{\prime} \in M_{c b}^{r}\left(L^{1}(\mathbb{G})\right)$. 
As is well-known in the group case (see $[34,74,76,98])$, for any $\hat{b}^{\prime} \in M_{c b}^{r}\left(L^{1}(\mathbb{G})\right)$, the map $\Theta^{r}\left(\hat{b}^{\prime}\right)$ extends to a normal completely bounded $L^{\infty}(\widehat{\mathbb{G}})$-bimodule map on $\mathcal{B}\left(L^{2}(\mathbb{G})\right)$ which leaves $L^{\infty}(\mathbb{G})$ globally invariant [53, Theorem 4.5], and the corresponding representation

$$
\Theta^{r}: M_{c b}^{r}\left(L^{1}(\mathbb{G})\right) \cong \mathcal{C B}_{L^{\infty}(\widehat{\mathbb{G}})}^{\sigma, L^{\infty}(\mathbb{G})}\left(\mathcal{B}\left(L^{2}(\mathbb{G})\right)\right)
$$

yields a completely isometric isomorphism of completely contractive Banach algebras. By duality, we obtain

$$
\widehat{\Theta}^{r}: M_{c b}^{r}\left(L^{1}(\widehat{\mathbb{G}})\right) \cong \mathcal{C B}_{L^{\infty}(\mathbb{G})}^{\sigma, L^{\infty}(\widehat{\mathbb{G}})}\left(\mathcal{B}\left(L^{2}(\mathbb{G})\right)\right)
$$

so the above representation framework allows one to study the actions of $\mathbb{G}$ and $\widehat{\mathbb{G}}$ on the single space $\mathcal{B}\left(L^{2}(\mathbb{G})\right)$. One manifestation of this duality is the following commutation theorem, where for a subset $\mathcal{S}$ of $\mathcal{C} \mathcal{B}\left(\mathcal{B}\left(L^{2}(\mathbb{G})\right)\right)$, we denote its commutant in $\mathcal{C B}\left(\mathcal{B}\left(L^{2}(\mathbb{G})\right)\right)$ by $\mathcal{S}^{c}$.

Theorem 3.7.3. [53, Theorem 5.1] Let $\mathbb{G}$ be a locally compact quantum group. Then

$$
\widehat{\Theta}^{r}\left(M_{c b}^{r}\left(L^{1}(\widehat{\mathbb{G}})\right)\right)=\Theta^{r}\left(M_{c b}^{r}\left(L^{1}(\mathbb{G})\right)\right)^{c} \cap \mathcal{C B}_{L^{\infty}(\mathbb{G})}^{\sigma}\left(\mathcal{B}\left(L^{2}(\mathbb{G})\right)\right)
$$

The corresponding result for completely bounded left multipliers takes the following form, and can be proved by combining [53, Theorem 5.1] with [49, Theorem 4.9].

Theorem 3.7.4. Let $\mathbb{G}$ be a locally compact quantum group. Then

$$
\widehat{\Theta}^{l}\left(M_{c b}^{l}\left(L^{1}(\widehat{\mathbb{G}})\right)\right)=\mathcal{U}^{*} \Theta^{r}\left(M_{c b}^{r}\left(L^{1}(\mathbb{G})\right)\right)^{c} \mathcal{U} \cap \mathcal{C B}_{L^{\infty}(\mathbb{G})^{\prime}}^{\sigma}\left(\mathcal{B}\left(L^{2}(\mathbb{G})\right)\right)
$$

where $\mathcal{U}$ is conjugation with the unitary $U=\widehat{J} J$. 


\section{Chapter 4}

\section{Amenability and Injectivity}

In this chapter we introduce and study versions of injectivity of $L^{\infty}(\widehat{\mathbb{G}})$ that capture fundamental properties of $\mathbb{G}$, such as amenability, compactness, and co-commutativity. The underlying idea is to refine injectivity through a covariance condition, by which we mean the existence of a conditional expectation respecting the natural $\mathcal{T}\left(L^{2}(\mathbb{G})\right)$ -

module of $\mathcal{B}\left(L^{2}(\mathbb{G})\right)$ or the $L^{1}(\widehat{\mathbb{G}})$-module structure of $L^{\infty}(\widehat{\mathbb{G}})$. We then relate this concept to that of injectivity in the category of operator modules.

\section{$4.1 \mathcal{T}\left(L^{2}(\mathbb{G})\right)$}

Let $\mathbb{G}$ be a locally compact quantum group. The right fundamental unitary $V$ of $\mathbb{G}$ induces a co-associative co-multiplication

$$
\Gamma^{r}: \mathcal{B}\left(L^{2}(\mathbb{G})\right) \ni T \mapsto V(T \otimes 1) V^{*} \in \mathcal{B}\left(L^{2}(\mathbb{G})\right) \bar{\otimes} \mathcal{B}\left(L^{2}(\mathbb{G})\right),
$$

and the restriction of $\Gamma^{r}$ to $L^{\infty}(\mathbb{G})$ yields the original co-multiplication $\Gamma$ on $L^{\infty}(\mathbb{G})$. The pre-adjoint of $\Gamma^{r}$ induces an associative completely contractive multiplication on $\mathcal{T}\left(L^{2}(\mathbb{G})\right)$, defined by

$$
\triangleright: \mathcal{T}\left(L^{2}(\mathbb{G})\right) \widehat{\otimes} \mathcal{T}\left(L^{2}(\mathbb{G})\right) \ni \omega \otimes \tau \mapsto \omega \triangleright \tau=\Gamma_{*}^{r}(\omega \otimes \tau) \in \mathcal{T}\left(L^{2}(\mathbb{G})\right) .
$$


Since $\Gamma^{r}$ is a complete isometry, it follows that $\Gamma_{*}^{r}$ is a complete quotient map, so we have

$$
\mathcal{T}\left(L^{2}(\mathbb{G})\right)=\left\langle\mathcal{T}\left(L^{2}(\mathbb{G})\right) \triangleright \mathcal{T}\left(L^{2}(\mathbb{G})\right)\right\rangle
$$

Analogously, the left fundamental unitary $W$ of $\mathbb{G}$ induces a co-associative co-multiplication

$$
\Gamma^{l}: \mathcal{B}\left(L^{2}(\mathbb{G})\right) \ni T \mapsto W^{*}(1 \otimes T) W \in \mathcal{B}\left(L^{2}(\mathbb{G})\right) \bar{\otimes} \mathcal{B}\left(L^{2}(\mathbb{G})\right)
$$

and the restriction of $\Gamma^{l}$ to $L^{\infty}(\mathbb{G})$ is also equal to $\Gamma$. The pre-adjoint of $\Gamma^{l}$ induces another associative completely contractive multiplication

$$
\triangleleft: \mathcal{T}\left(L^{2}(\mathbb{G})\right) \widehat{\otimes} \mathcal{T}\left(L^{2}(\mathbb{G})\right) \ni \omega \otimes \tau \mapsto \omega \triangleleft \tau=\Gamma_{*}^{l}(\omega \otimes \tau) \in \mathcal{T}\left(L^{2}(\mathbb{G})\right)
$$

These two products on $\mathcal{T}\left(L^{2}(\mathbb{G})\right)$ are quite different in general. It is known that $\left(\mathcal{T}\left(L^{2}(\mathbb{G})\right), \triangleright\right)$ is always left faithful, and right faithful if and only if $\mathbb{G}$ is trivial. Similarly, $\left(\mathcal{T}\left(L^{2}(\mathbb{G})\right), \triangleleft\right)$ is always right faithful, and is left faithful if and only if $\mathbb{G}$ is trivial (cf. [49]).

The above Banach algebra structure on $\mathcal{T}\left(L^{2}(\mathbb{G})\right)$ was introduced in the commutative case by Neufang [74], and was further studied in [1, 76, 83]. The general case has been investigated in $[49,50,56]$. In particular, it was shown in [49, Lemma 5.2] that the pre-annihilator $L^{\infty}(\mathbb{G})_{\perp}$ of $L^{\infty}(\mathbb{G})$ in $\mathcal{T}\left(L^{2}(\mathbb{G})\right)$ is a norm closed two sided ideal in $\left(\mathcal{T}\left(L^{2}(\mathbb{G})\right), \triangleright\right)$ and $\left(\mathcal{T}\left(L^{2}(\mathbb{G})\right), \triangleleft\right)$, respectively, and the complete quotient map

$$
\pi: \mathcal{T}\left(L^{2}(\mathbb{G})\right) \ni \omega \mapsto f=\left.\omega\right|_{L^{\infty}(\mathbb{G})} \in L^{1}(\mathbb{G})
$$

is a completely contractive algebra homomorphism from $\left(\mathcal{T}\left(L^{2}(\mathbb{G})\right), \triangleright\right)$, respectively, $\left(\mathcal{T}\left(L^{2}(\mathbb{G})\right), \triangleleft\right)$, onto $L^{1}(\mathbb{G})$. Therefore, we have the completely isometric Banach 
algebra identifications

$$
\left(L^{1}(\mathbb{G}), \star\right) \cong\left(\mathcal{T}\left(L^{2}(\mathbb{G})\right), \triangleright\right) / L^{\infty}(\mathbb{G})_{\perp} \quad \text { and } \quad\left(L^{1}(\mathbb{G}), \star\right) \cong\left(\mathcal{T}\left(L^{2}(\mathbb{G})\right), \triangleleft\right) / L^{\infty}(\mathbb{G})_{\perp}
$$

This allows us to view each of $\left(\mathcal{T}\left(L^{2}(\mathbb{G})\right), \triangleright\right)$ and $\left(\mathcal{T}\left(L^{2}(\mathbb{G})\right), \triangleleft\right)$ as a lifting of $\left(L^{1}(\mathbb{G}), \star\right)$.

Letting $\widehat{J}$ denote the conjugate linear isomorphism of $L^{2}(\mathbb{G})$ arising from the GNS construction of the left Haar weight $\widehat{\varphi}$, we obtain a canonical extension of the unitary antipode to a ${ }^{*}$-anti-automorphism $R: \mathcal{B}\left(L^{2}(\mathbb{G})\right) \rightarrow \mathcal{B}\left(L^{2}(\mathbb{G})\right)$, given by $R(T)=\widehat{J} T^{*} \widehat{J}, T \in \mathcal{B}\left(L^{2}(\mathbb{G})\right)$. The extended antipode maps $L^{\infty}(\mathbb{G})$ and $L^{\infty}(\widehat{\mathbb{G}})$ onto $L^{\infty}(\mathbb{G})$ and $L^{\infty}\left(\widehat{\mathbb{G}}^{\prime}\right)$, respectively, and satisfies the generalized antipode relations; that is,

$$
(R \otimes R) \circ \Gamma^{r}=\Sigma \circ \Gamma^{l} \circ R \text { and }(R \otimes R) \circ \Gamma^{l}=\Sigma \circ \Gamma^{r} \circ R,
$$

where $\Sigma$ is the flip map on $\mathcal{B}\left(L^{2}(\mathbb{G})\right) \bar{\otimes} \mathcal{B}\left(L^{2}(\mathbb{G})\right)$. At the level of $\mathcal{T}\left(L^{2}(\mathbb{G})\right)$, the relations (4.3) mean

$$
R_{*}(\omega \triangleright \tau)=R_{*}(\tau) \triangleleft R_{*}(\omega) \text { and } \quad R_{*}(\omega \triangleleft \tau)=R_{*}(\tau) \triangleright R_{*}(\omega)
$$

for all $\omega, \tau \in \mathcal{T}\left(L^{2}(\mathbb{G})\right)$. We may therefore pass between the left and right products using $R$, and as a result, we will often focus on the right product $\triangleright$ throughout the thesis, leaving the corresponding result to the reader to establish.

Example 4.1.1. Let $\mathbb{G}_{a}=\left(L^{\infty}(G), \Gamma_{a}, \varphi_{a}, \psi_{a}\right)$ be a commutative locally compact quantum group. Then, as shown in [74], we have

$$
\omega \triangleright_{a} \tau=\int_{G} \rho(s)^{*} \omega \rho(s) \pi_{a}(\tau)(s) d s \text { and } \omega \triangleleft_{a} \tau=\int_{G} \lambda(s) \tau \lambda(s)^{*} \pi_{a}(\omega)(s) d s
$$

for any $\omega, \tau \in \mathcal{T}\left(L^{2}(G)\right)$. Hence, in the commutative case we may literally view $\triangleright_{a}$ and $\triangleleft_{a}$ as a lifting of convolution from $L^{1}(G)$ to $\mathcal{T}\left(L^{2}(G)\right)$. 
Example 4.1.2. Let $\mathbb{G}_{s}=\left(\mathcal{L}(G), \Gamma_{s}, \varphi_{s}\right)$ be a co-commutative quantum group with $G$ discrete. Then using the canonical orthonormal basis $\left(\delta_{s}\right)_{s \in G}$ of $\ell^{2}(G)$, one may easily verify that

$$
\omega \triangleright_{s} \tau=\left[\pi_{s}(\tau)\left(r t^{-1}\right)\right]_{r, t} \circ_{S} \omega \text { and } \omega \triangleleft_{s} \tau=\left[\pi_{s}(\omega)\left(r^{-1} t\right)\right]_{r, t} \circ_{S} \tau
$$

where $\left[\pi_{s}(\tau)\left(r t^{-1}\right)\right]_{r, t}$ is the correlation matrix obtained from $\pi_{s}(\tau) \in A(G)$, and $\circ_{S}$ is the Schur product with respect to the canonical orthonormal basis. We may therefore view the products $\triangleright_{s}$ and $\triangleleft_{s}$, at least concretely in the discrete case, as a lifting of pointwise multiplication from $A(G)$ to $\mathcal{T}\left(\ell^{2}(G)\right)$.

Since $L^{2}(\mathbb{G})=L^{2}(\widehat{\mathbb{G}})$ for any locally compact quantum group $\mathbb{G}$, applying the above construction to the co-multiplication $\widehat{\Gamma}$ on $L^{\infty}(\widehat{\mathbb{G}})$ yields two dual products

$$
\begin{aligned}
& \widehat{\triangleright}: \mathcal{T}\left(L^{2}(\mathbb{G})\right) \widehat{\otimes} \mathcal{T}\left(L^{2}(\mathbb{G})\right) \ni \omega \otimes \tau \mapsto \omega \widehat{\triangleright} \tau=\widehat{\Gamma}_{*}^{r}(\omega \otimes \tau) \in \mathcal{T}\left(L^{2}(\mathbb{G})\right), \\
& \widehat{\triangleleft}: \mathcal{T}\left(L^{2}(\mathbb{G})\right) \widehat{\otimes} \mathcal{T}\left(L^{2}(\mathbb{G})\right) \ni \omega \otimes \tau \mapsto \omega \widehat{\triangleleft} \tau=\widehat{\Gamma}_{*}^{l}(\omega \otimes \tau) \in \mathcal{T}\left(L^{2}(\mathbb{G})\right) .
\end{aligned}
$$

As with the case of completely bounded multipliers, lifting convolution to $\mathcal{T}\left(L^{2}(\mathbb{G})\right)$ allows one to study properties of $\mathbb{G}$ and $\widehat{\mathbb{G}}$ as well as their interactions a single space. The advantage of $\mathcal{T}\left(L^{2}(\mathbb{G})\right)$ is that certain interactions have important homological consequences. One such interaction was shown in [56], and states that the dual products anti-commute.

Theorem 4.1.3. [56, Theorem 3.3] Let $\mathbb{G}$ be a locally compact quantum group. Then for every $\rho, \omega, \tau \in \mathcal{T}\left(L^{2}(\mathbb{G})\right)$ we have

$$
(\rho \triangleright \omega) \widehat{\triangleright} \tau=(\rho \widehat{\triangleright} \tau) \triangleright \omega .
$$

Equation (4.4) is a manifestation of the commutation theorem for completely bounded multipliers $(3.9)$ at the level of $\mathcal{T}\left(L^{2}(\mathbb{G})\right.$ ). Below we derive an important consequence (Proposition 4.1.6) that will be used many times throughout this chapter. 
For now, we show that in the Kac algebra setting we may further interpret the above commutation relation at the level of the co-multiplications $\Gamma$ and $\widehat{\Gamma}$. A similar result was recently obtained by Kasprzak and Soltan using different techniques [58, Proposition 6.3].

Theorem 4.1.4. Let $\mathbb{G}$ be a Kac algebra. Then

$$
\Gamma\left(L^{\infty}(\mathbb{G})\right)=(U \otimes 1) \widehat{\Gamma}\left(L^{\infty}(\widehat{\mathbb{G}})\right)^{\prime}\left(U^{*} \otimes 1\right) \cap L^{\infty}(\mathbb{G}) \bar{\otimes} L^{\infty}(\mathbb{G}),
$$

where $U=\widehat{J} J$.

Proof. Throughout the proof we denote conjugation with $U$ by $\mathcal{U}$. Let $b \in M_{c b}^{l}\left(L^{1}(\widehat{\mathbb{G}})\right)$, then by [21, Proposition 6.1], for any $\xi, \eta, \alpha, \beta \in L^{2}(\mathbb{G})$ we have

$$
\left\langle\widehat{\Theta}^{l}(b)(|\xi\rangle\langle\eta|) \alpha, \beta\right\rangle=\left\langle(\mathrm{id} \otimes R) \Gamma(b), \omega_{\alpha, \eta} \otimes \omega_{\xi, \beta}\right\rangle .
$$

We remark that the above formula involving the unitary antipode is specific to the case of Kac algebras (see [21, Proposition 6.1]). By an unpublished result of Haagerup [41], there exist nets $\left(a_{i}\right)_{i \in I}$ and $\left(b_{i}\right)_{i \in I}$ in $L^{\infty}(\mathbb{G})$ such that $\sum_{i \in I} a_{i} a_{i}^{*}, \sum_{i \in I} b_{i}^{*} b_{i}$ converge weak* in $L^{\infty}(\mathbb{G})$ and

$$
\widehat{\Theta}^{l}(b)(T)=\sum_{i \in I} a_{i} T b_{i}, \quad T \in \mathcal{B}\left(L^{2}(\mathbb{G})\right)
$$

where the series converges weak* in $\mathcal{B}\left(L^{2}(\mathbb{G})\right)$. Using this with equation (4.5) gives

$$
\sum_{i \in I} a_{i} \otimes b_{i}=\Sigma \circ(\mathrm{id} \otimes R) \Gamma(b)=(\mathrm{id} \otimes R) \Gamma(R(b)) \in L^{\infty}(\mathbb{G}) \bar{\otimes} L^{\infty}(\mathbb{G}) .
$$

By duality, for any $\widehat{b} \in M_{c b}^{l}\left(L^{1}(\mathbb{G})\right)$ there exist nets $\left(\hat{x}_{j}\right)_{j \in J}$ and $\left(\hat{y}_{j}\right)_{j \in J}$ in $L^{\infty}(\widehat{\mathbb{G}})$ such that $\sum_{j \in J} \hat{x}_{j} \hat{x}_{j}^{*}, \sum_{j \in J} \hat{y}_{j}^{*} \hat{y}_{j}$ converge weak ${ }^{*}$ in $L^{\infty}(\widehat{\mathbb{G}})$,

$$
\Theta^{l}(\hat{b})(T)=\sum_{j \in J} \widehat{x}_{j} T \widehat{y}_{j}, \quad T \in \mathcal{B}\left(L^{2}(\mathbb{G})\right),
$$


and

$$
\left.\sum_{j \in J} \widehat{x}_{j} \otimes \widehat{y}_{j}=\Sigma \circ(\operatorname{id} \otimes \widehat{R}) \widehat{\Gamma} \widehat{b}\right)=(\operatorname{id} \otimes \widehat{R}) \widehat{\Gamma}(\widehat{R}(\widehat{b})) \in L^{\infty}(\widehat{\mathbb{G}}) \bar{\otimes} L^{\infty}(\widehat{\mathbb{G}}) .
$$

Noting that $\mathcal{U}=R \circ \widehat{R}=\widehat{R} \circ R$, we obtain

$$
\begin{aligned}
& \left\langle\Theta^{l}(\widehat{b}) \circ \mathcal{U} \circ \widehat{\Theta}^{l}(b) \circ \mathcal{U}^{*}(|\xi\rangle\langle\eta|) \alpha, \beta\right\rangle \\
& =\sum_{j \in J} \sum_{i \in I}\left\langle\hat{x}_{j} U a_{i} U^{*} \mid \xi\right\rangle\left\langle\eta \mid U b_{i} U^{*} \widehat{y}_{j} \alpha, \beta\right\rangle \\
& =\sum_{j \in J} \sum_{i \in I}\left\langle\widehat{x}_{j} U a_{i} U^{*} \otimes U b_{i} U^{*} \widehat{y}_{j}, \omega_{\xi, \beta} \otimes \omega_{\alpha, \eta}\right\rangle \\
& =\sum_{j \in J} \sum_{i \in I}\left\langle\left(\widehat{x}_{j} \otimes \widehat{R}\left(\widehat{y}_{j}\right)\right)\left(U a_{i} U^{*} \otimes \widehat{R}\left(U b_{i} U^{*}\right)\right), \omega_{\xi, \beta} \otimes \omega_{\alpha, \eta} \circ \widehat{R}\right\rangle \\
& =\sum_{j \in J} \sum_{i \in I}\left\langle\left(\widehat{x}_{j} \otimes \widehat{R}\left(\widehat{y}_{j}\right)\right)\left((U \otimes 1)(\mathrm{id} \otimes R)\left(a_{i} \otimes b_{i}\right)\left(U^{*} \otimes 1\right)\right), \omega_{\xi, \beta} \otimes \omega_{\alpha, \eta} \circ \widehat{R}\right\rangle \\
& =\sum_{j \in J}\left\langle\left(\widehat{x}_{j} \otimes \widehat{R}\left(\widehat{y}_{j}\right)\right)\left((U \otimes 1) \Gamma(R(b))\left(U^{*} \otimes 1\right)\right), \omega_{\xi, \beta} \otimes \omega_{\alpha, \eta} \circ \widehat{R}\right\rangle \\
& =\left\langle\widehat{\Gamma}(\widehat{R}(\widehat{b}))\left((U \otimes 1) \Gamma(R(b))\left(U^{*} \otimes 1\right)\right), \omega_{\xi, \beta} \otimes \omega_{\alpha, \eta} \circ \widehat{R}\right\rangle .
\end{aligned}
$$

On the other hand, $\Theta^{l}(\widehat{b}) \circ \mathcal{U} \circ \widehat{\Theta}^{l}(b) \circ \mathcal{U}^{*}=\mathcal{U} \circ \widehat{\Theta}^{l}(b) \circ \mathcal{U}^{*} \circ \Theta^{l}(\widehat{b})$ by (3.10), and a similar computation gives

$$
\left\langle\Theta^{l}(\widehat{b}) \circ \widehat{\Theta}^{l}(b)(|\xi\rangle\langle\eta|) \alpha, \beta\right\rangle=\left\langle(U \otimes 1) \Gamma(R(b))\left(U^{*} \otimes 1\right) \widehat{\Gamma}(\widehat{R}(\widehat{b})), \omega_{\eta, \beta} \otimes \omega_{\alpha, \eta} \circ \widehat{R}\right\rangle .
$$

By density of $\omega_{\eta, \beta} \otimes \omega_{\alpha, \eta} \circ \widehat{R}$ in $\mathcal{T}\left(L^{2}(\mathbb{G})\right) \widehat{\otimes} \mathcal{T}\left(L^{2}(\mathbb{G})\right)$, it follows that

$$
\left[\widehat{\Gamma}(\widehat{R}(\widehat{b})),(U \otimes 1) \Gamma(R(b))\left(U^{*} \otimes 1\right)\right]=0 \Leftrightarrow\left[(U \otimes 1) \widehat{\Gamma}(\hat{b})\left(U^{*} \otimes 1\right), \Gamma(b)\right]=0
$$

for all $b \in M_{c b}^{l}\left(L^{1}(\widehat{\mathbb{G}})\right)$ and $\widehat{b} \in M_{c b}^{l}\left(L^{1}(\mathbb{G})\right)$ (the equivalence follows from the antipode relations). By normality and weak* density of $M_{c b}^{l}\left(L^{1}(\mathbb{G})\right)$ and $M_{c b}^{l}\left(L^{1}(\widehat{\mathbb{G}})\right)$ in $L^{\infty}(\widehat{\mathbb{G}})$ 
and $L^{\infty}(\mathbb{G})$, respectively, we obtain the inclusion

$$
\Gamma\left(L^{\infty}(\mathbb{G})\right) \subseteq(U \otimes 1) \widehat{\Gamma}\left(L^{\infty}(\widehat{\mathbb{G}})\right)^{\prime}\left(U^{*} \otimes 1\right) \cap L^{\infty}(\mathbb{G}) \bar{\otimes} L^{\infty}(\mathbb{G})
$$

Conversely, suppose $X \in(U \otimes 1) \widehat{\Gamma}\left(L^{\infty}(\widehat{\mathbb{G}})\right)^{\prime}\left(U^{*} \otimes 1\right) \cap L^{\infty}(\mathbb{G}) \bar{\otimes} L^{\infty}(\mathbb{G})$. Then, unfolding the co-multiplication we obtain

$$
\widehat{W}\left(U^{*} \otimes 1\right) X(U \otimes 1) \widehat{W}^{*}(1 \otimes \widehat{x})=(1 \otimes \widehat{x}) \widehat{W}\left(U^{*} \otimes 1\right) X(U \otimes 1) \widehat{W}^{*}
$$

for every $\widehat{x} \in L^{\infty}(\widehat{\mathbb{G}})$. Since $\widehat{W} \in L^{\infty}(\widehat{\mathbb{G}}) \bar{\otimes} L^{\infty}(\mathbb{G})$, it follows that

$$
(\rho \otimes \mathrm{id})\left(\widehat{W}\left(U^{*} \otimes 1\right) X(U \otimes 1) \widehat{W}^{*}\right) \in L^{\infty}\left(\widehat{\mathbb{G}}^{\prime}\right) \cap L^{\infty}(\mathbb{G})=\mathbb{C} 1
$$

for every $\rho \in \mathcal{T}\left(L^{2}(\mathbb{G})\right)$. Hence, $\widehat{W}\left(U^{*} \otimes 1\right) X(U \otimes 1) \widehat{W}^{*}=T \otimes 1$ for some $T \in$ $\mathcal{B}\left(L^{2}(\mathbb{G})\right)$, and formula $(3.5)$ gives $X=V\left(U T U^{*} \otimes 1\right) V^{*}=\Gamma^{r}\left(U T U^{*}\right)$, implying that $X \in \Gamma^{r}\left(\mathcal{B}\left(L^{2}(\mathbb{G})\right)\right) \cap L^{\infty}(\mathbb{G}) \bar{\otimes} L^{\infty}(\mathbb{G})$. But then $(\Gamma \otimes \mathrm{id})(X)=(\mathrm{id} \otimes \Gamma)(X)$ by co-associativity, which forces $X \in \Gamma\left(L^{\infty}(\mathbb{G})\right)$ by [107, Theorem 2.7].

For a locally compact quantum group $\mathbb{G}$, the multiplication $\triangleright$ defines a completely contractive $\left(\mathcal{T}\left(L^{2}(\mathbb{G})\right), \triangleright\right)$-bimodule structure on $\mathcal{B}\left(L^{2}(\mathbb{G})\right)$ via

$\mathcal{B}\left(L^{2}(\mathbb{G})\right) \widehat{\otimes} \mathcal{T}\left(L^{2}(\mathbb{G})\right) \ni(T, \omega) \mapsto T \triangleright \omega=(\omega \otimes \mathrm{id}) V(T \otimes 1) V^{*} \in L^{\infty}(\mathbb{G}) \subseteq \mathcal{B}\left(L^{2}(\mathbb{G})\right) ;$ $\mathcal{T}\left(L^{2}(\mathbb{G})\right) \widehat{\otimes} \mathcal{B}\left(L^{2}(\mathbb{G})\right) \ni(\omega, T) \mapsto \omega \triangleright T=(\mathrm{id} \otimes \omega) V(T \otimes 1) V^{*} \in \mathcal{B}\left(L^{2}(\mathbb{G})\right)$.

Note that since $V \in L^{\infty}\left(\widehat{\mathbb{G}}^{\prime}\right) \bar{\otimes} L^{\infty}(\mathbb{G})$, the (restricted) bimodule action on $L^{\infty}(\widehat{\mathbb{G}})$ becomes rather trivial. Indeed, for $\hat{x} \in L^{\infty}(\widehat{\mathbb{G}})$ and $\omega \in \mathcal{T}\left(L^{2}(\mathbb{G})\right)$

$$
\hat{x} \triangleright \omega=\langle\omega, \hat{x}\rangle 1 \text { and } \omega \triangleright \hat{x}=\langle\omega, 1\rangle \hat{x} .
$$

The multiplication $\triangleleft$ defines, analogously, a completely contractive $\left(\mathcal{T}\left(L^{2}(\mathbb{G})\right), \triangleleft\right)$ - 
bimodule structure on $\mathcal{B}\left(L^{2}(\mathbb{G})\right)$ via

$\mathcal{T}\left(L^{2}(\mathbb{G})\right) \widehat{\otimes} \mathcal{B}\left(L^{2}(\mathbb{G})\right) \ni(\omega, T) \mapsto \omega \triangleleft T=(\mathrm{id} \otimes \omega) W^{*}(1 \otimes T) W \in L^{\infty}(\mathbb{G}) \subseteq \mathcal{B}\left(L^{2}(\mathbb{G})\right) ;$

$\mathcal{B}\left(L^{2}(\mathbb{G})\right) \widehat{\otimes} \mathcal{T}\left(L^{2}(\mathbb{G})\right) \ni(T, \omega) \mapsto T \triangleleft \omega=(\omega \otimes \mathrm{id}) W^{*}(1 \otimes T) W \in \mathcal{B}\left(L^{2}(\mathbb{G})\right)$.

In particular, for $x \in L^{\infty}(\mathbb{G})$ and $\omega \in \mathcal{T}\left(L^{2}(\mathbb{G})\right)$, denoting $f=\left.\omega\right|_{L^{\infty}(\mathbb{G})}$, we have

$$
x \triangleright \omega=x \triangleleft \omega=x \star f \quad \text { and } \quad \omega \triangleleft x=\omega \triangleright x=f \star x .
$$

As above, we see that the bimodule actions of $\left(\mathcal{T}\left(L^{2}(\mathbb{G})\right), \triangleright\right)$ and $\left(\mathcal{T}\left(L^{2}(\mathbb{G})\right), \triangleleft\right)$ on $\mathcal{B}\left(L^{2}(\mathbb{G})\right)$ are liftings of the usual bimodule action of $L^{1}(\mathbb{G})$ on $L^{\infty}(\mathbb{G})$. When $\mathbb{G}=\mathbb{G}_{a}$ is commutative, the right $\left(\mathcal{T}\left(L^{2}(\mathbb{G})\right), \triangleright_{a}\right)$-action and left $\left(\mathcal{T}\left(L^{2}(\mathbb{G})\right), \triangleleft_{a}\right)$-action take the following form (see [74]):

$$
T \triangleright_{a} \omega(s)=\left\langle\rho(s) T \rho(s)^{*}, \omega\right\rangle \quad \text { and } \quad \omega \triangleleft_{a} T(s)=\left\langle\lambda(s)^{*} T \lambda(s), \omega\right\rangle, \quad s \in G .
$$

Motivated by the group setting, the subspaces $\operatorname{LUC}(\mathbb{G})$ and $\operatorname{RUC}(\mathbb{G})$ of $L^{\infty}(\mathbb{G})$ are defined by $[48,93]$

$$
\operatorname{LUC}(\mathbb{G})=\left\langle L^{\infty}(\mathbb{G}) \star L^{1}(\mathbb{G})\right\rangle \text { and } \operatorname{RUC}(\mathbb{G})=\left\langle L^{1}(\mathbb{G}) \star L^{\infty}(\mathbb{G})\right\rangle
$$

It was shown by Runde in $[93$, Theorem 2.4$]$ that $\operatorname{LUC}(\mathbb{G})$ and $\mathrm{RUC}(\mathbb{G})$ are operator systems in $L^{\infty}(\mathbb{G})$ such that

$$
\mathbb{C}_{0}(\mathbb{G}) \subseteq \operatorname{LUC}(\mathbb{G}), \operatorname{RUC}(\mathbb{G}) \subseteq M\left(C_{0}(\mathbb{G})\right)
$$

By definition, $\operatorname{LUC}\left(\mathbb{G}_{a}\right)$ (respectively, $\operatorname{RUC}\left(\mathbb{G}_{a}\right)$ ) is the usual space $\operatorname{LUC}(G)$ (respectively, $\operatorname{RUC}(G)$ ) of bounded left (respectively, right) uniformly continuous functions on $G$, and $\operatorname{LUC}\left(\mathbb{G}_{s}\right)=\operatorname{RUC}\left(\mathbb{G}_{s}\right)$ is the space $\operatorname{UCB}(\widehat{G})$ of uniformly continuous bounded linear functionals on $A(G)$ introduced by Granirer [37]. Using the extended 
module actions of $\mathcal{T}\left(L^{2}(\mathbb{G})\right)$ on $\mathcal{B}\left(L^{2}(\mathbb{G})\right)$, it was shown in [49, Proposition 5.3] that

$$
\begin{aligned}
& \operatorname{LUC}(\mathbb{G})=\left\langle\operatorname{LUC}(\mathbb{G}) \star L^{1}(\mathbb{G})\right\rangle=\left\langle\mathcal{B}\left(L^{2}(\mathbb{G})\right) \triangleright \mathcal{T}\left(L^{2}(\mathbb{G})\right)\right\rangle \\
& \operatorname{RUC}(\mathbb{G})=\left\langle L^{1}(\mathbb{G}) \star \operatorname{RUC}(\mathbb{G})\right\rangle=\left\langle\mathcal{T}\left(L^{2}(\mathbb{G})\right) \triangleleft \mathcal{B}\left(L^{2}(\mathbb{G})\right)\right\rangle
\end{aligned}
$$

When $\mathbb{G}$ is co-amenable, $\left(\mathcal{T}\left(L^{2}(\mathbb{G})\right), \triangleright\right)$ (respectively, $\left.\left(\mathcal{T}\left(L^{2}(\mathbb{G})\right), \triangleleft\right)\right)$ has a contractive right (respectively, left) approximate identity [49, Proposition 5.4]. In this case, Cohen's factorization theorem yields the equalities

$$
\operatorname{LUC}(\mathbb{G})=\mathcal{B}\left(L^{2}(\mathbb{G})\right) \triangleright \mathcal{T}\left(L^{2}(\mathbb{G})\right) \quad \text { and } \quad \operatorname{RUC}(\mathbb{G})=\mathcal{T}\left(L^{2}(\mathbb{G})\right) \triangleleft \mathcal{B}\left(L^{2}(\mathbb{G})\right)
$$

For every locally compact quantum group $\mathbb{G}$, we have the left and right Arens products $\square$ and $\diamond$ on $L^{\infty}(\mathbb{G})^{*}=L^{1}(\mathbb{G})^{* *}$. Then $\left(L^{\infty}(\mathbb{G})^{*}, \square\right)$ and $\left(L^{\infty}(\mathbb{G})^{*}, \diamond\right)$ are completely contractive Banach algebras. Given $m \in \operatorname{LUC}(\mathbb{G})^{*}$, we define a bounded linear map $m_{L}$ on $L^{\infty}(\mathbb{G})$ by

$$
m_{L}: L^{\infty}(\mathbb{G}) \ni x \mapsto m \square x \in L^{\infty}(\mathbb{G}) .
$$

This map is completely bounded, with $\left\|m_{L}\right\|_{c b} \leq\|m\|$, and a right $L^{1}(\mathbb{G})$-module map, since

$$
\langle m \square(x \star f), g\rangle=\langle m, x \star(f \star g)\rangle=\langle m \square x, f \star g\rangle=\langle(m \square x) \star f, g\rangle
$$

for all $x \in L^{\infty}(\mathbb{G})$ and $f, g \in L^{1}(\mathbb{G})$. Therefore, $m_{L}$ maps $\operatorname{LUC}(\mathbb{G})$ into $\operatorname{LUC}(\mathbb{G})$, and the left Arens product $\square$ on $L^{\infty}(\mathbb{G})^{*}$ induces a completely contractive multiplication on $\operatorname{LUC}(\mathbb{G})^{*}$, also denoted $\square$, so that the restriction

$$
\left.L^{\infty}(\mathbb{G})^{*} \ni m \mapsto m\right|_{\mathrm{LUC}(\mathbb{G})} \in \operatorname{LUC}(\mathbb{G})^{*}
$$

is a multiplicative complete quotient map from $\left(L^{\infty}(\mathbb{G})^{*}, \square\right)$ onto $\left(\operatorname{LUC}(\mathbb{G})^{*}, \square\right)$. 
Let $m \in \operatorname{LUC}(\mathbb{G})^{*}$. Then, as $\operatorname{LUC}(\mathbb{G})=\left\langle\mathcal{B}\left(L^{2}(\mathbb{G})\right) \triangleright \mathcal{T}\left(L^{2}(\mathbb{G})\right)\right\rangle$, the module map $m_{L}$ may be extended to a right $\left(\mathcal{T}\left(L^{2}(\mathbb{G})\right), \triangleright\right)$-module map $\Theta^{r}(m)$ on $\mathcal{B}\left(L^{2}(\mathbb{G})\right)$ via

$$
\left\langle\Theta^{r}(m)(T), \omega\right\rangle=\langle m, T \triangleright \omega\rangle=\left\langle m,(\omega \otimes \mathrm{id}) V(T \otimes 1) V^{*}\right\rangle
$$

for $T \in \mathcal{B}\left(L^{2}(\mathbb{G})\right)$ and $\omega \in \mathcal{T}\left(L^{2}(\mathbb{G})\right)$. In this case, we also have $\left\|\Theta^{r}(m)\right\|_{c b} \leq$ $\|m\|$, and if we let $\mathcal{C B}_{\mathcal{T}_{\triangleright}}\left(\mathcal{B}\left(L^{2}(\mathbb{G})\right)\right)$ denote the algebra of completely bounded right $\left(\mathcal{T}\left(L^{2}(\mathbb{G})\right), \triangleright\right)$-module maps on $\mathcal{B}\left(L^{2}(\mathbb{G})\right)$, it follows that

$$
\Theta^{r}: \operatorname{LUC}(\mathbb{G})^{*} \ni m \mapsto \Theta^{r}(m) \in \mathcal{C} \mathcal{B}_{\mathcal{T}_{\triangleright}}\left(\mathcal{B}\left(L^{2}(\mathbb{G})\right)\right)
$$

is a weak*-weak* continuous, injective, completely contractive algebra homomorphism [49, Proposition 6.5].

Remark 4.1.5. Since $\operatorname{LUC}(\mathbb{G}) \subseteq M\left(C_{0}(\mathbb{G})\right)$, one can show that the algebra $M(\mathbb{G})$ sits isometrically inside $\operatorname{LUC}(\mathbb{G})^{*}$ via

$$
\left.M(\mathbb{G}) \ni \mu \mapsto \widetilde{\mu}\right|_{\mathrm{LUC}(\mathbb{G})} \in \operatorname{LUC}(\mathbb{G})^{*},
$$

where $\widetilde{\mu} \in M\left(C_{0}(\mathbb{G})\right)^{*}$ is the unique strict extension of $\mu$ (see [49, Proposition 6.1]). When $\mathbb{G}$ is co-amenable, we have $M_{c b}^{r}\left(L^{1}(\mathbb{G})\right) \cong M(\mathbb{G})[49$, Theorem 4.2], and the restricted representation $\left.\Theta^{r}\right|_{M(\mathbb{G})}$ coincides with (3.8), justifying our common notation. In general, $M(\mathbb{G})$ sits completely contractively inside $M_{c b}^{r}\left(L^{1}(\mathbb{G})\right)$ [49, Proposition 4.1], and the restricted representations of $\operatorname{LUC}(\mathbb{G})^{*}$ and $M_{c b}^{r}\left(L^{1}(\mathbb{G})\right)$ agree on $M(\mathbb{G})$, so we will continue to denote both representations by $\Theta^{r}$, the particular case being clear from context.

Analogously, the right Arens product $\diamond$ induces a completely contractive Banach algebra structure on $\operatorname{RUC}(\mathbb{G})^{*}$, and there exists a weak*-weak* continuous, injective, 
completely contractive anti-homomorphism

$$
\Theta^{l}: \operatorname{RUC}(\mathbb{G})^{*} \rightarrow \mathcal{T}_{\triangleleft} \mathcal{C B}\left(\mathcal{B}\left(L^{2}(\mathbb{G})\right)\right)
$$

where $\mathcal{T}_{\triangleleft} \mathcal{C B}\left(\mathcal{B}\left(L^{2}(\mathbb{G})\right)\right)$ is the algebra of completely bounded left $\left(\mathcal{T}\left(L^{2}(\mathbb{G})\right), \triangleleft\right)$ module maps on $\mathcal{B}\left(L^{2}(\mathbb{G})\right)$.

In $[49$, Remark 7.4], the authors observe that for co-amenable $\mathbb{G}$ we have

$$
\mathcal{C} \mathcal{B}_{\mathcal{T}_{\triangleright}}\left(\mathcal{B}\left(L^{2}(\mathbb{G})\right)\right) \subseteq \mathcal{C B}_{L^{\infty}(\mathbb{G})}^{L^{\infty}(\mathbb{G})}\left(\mathcal{B}\left(L_{2}(\mathbb{G})\right)\right)
$$

where $\mathcal{C B}_{L^{\infty}(\widetilde{\mathbb{G}})}^{L^{\infty}(\mathbb{G})}\left(\mathcal{B}\left(L_{2}(\mathbb{G})\right)\right)$ is the algebra of completely bounded $L^{\infty}(\widehat{\mathbb{G}})$-bimodule maps on $\mathcal{B}\left(L^{2}(\mathbb{G})\right)$ that leave $L^{\infty}(\mathbb{G})$ globally invariant. As a corollary to the commutation relation (4.4), we can remove the co-amenability hypothesis in the above inclusion using the following "automatic" module property.

Proposition 4.1.6. Let $\mathbb{G}$ be locally compact quantum group. Then

$$
\mathcal{C B}_{\mathcal{T}_{\triangleright}}\left(\mathcal{B}\left(L^{2}(\mathbb{G})\right)\right) \subseteq \mathcal{T}_{\bar{\triangleright}} \mathcal{C B}\left(\mathcal{B}\left(L^{2}(\mathbb{G})\right)\right)
$$

Proof. Let $\Phi \in \mathcal{C B}_{\mathcal{T}_{\triangleright}}\left(\mathcal{B}\left(L^{2}(\mathbb{G})\right)\right)$, and fix $\rho \in \mathcal{T}\left(L^{2}(\mathbb{G})\right)$ and $T \in \mathcal{B}\left(L^{2}(\mathbb{G})\right)$. Then for any $\omega, \tau \in \mathcal{T}\left(L^{2}(\mathbb{G})\right)$, we have

$\langle(\rho \widehat{\triangleright} T) \triangleright \tau, \omega\rangle=\langle\rho \widehat{\triangleright} T, \tau \triangleright \omega\rangle=\langle T,(\tau \triangleright \omega) \widehat{\triangleright} \rho\rangle=\langle T,(\tau \widehat{\triangleright} \rho) \triangleright \omega\rangle=\langle T \triangleright(\tau \widehat{\triangleright} \rho), \omega\rangle$.

Thus,

$$
\begin{aligned}
\langle\Phi(\rho \widehat{\triangleright} T), \tau \triangleright \omega\rangle & =\langle\Phi(\rho \widehat{\triangleright} T) \triangleright \tau, \omega\rangle=\langle\Phi((\rho \widehat{\triangleright} T) \triangleright \tau), \omega\rangle \\
& =\langle\Phi(T \triangleright(\tau \widehat{\triangleright} \rho)), \omega\rangle=\langle\Phi(T) \triangleright(\tau \widehat{\triangleright} \rho), \omega\rangle \\
& =\langle\Phi(T),(\tau \widehat{\triangleright} \rho) \triangleright \omega\rangle=\langle\Phi(T),(\tau \triangleright \omega) \widehat{\triangleright} \rho\rangle \\
& =\langle\rho \widehat{\triangleright} \Phi(T), \tau \triangleright \omega\rangle .
\end{aligned}
$$


By (4.1), it follows that $\Phi(\rho \widehat{\triangleright} T)=\rho \widehat{\triangleright} \Phi(T)$, as required.

Corollary 4.1.7. For any locally compact quantum group $\mathbb{G}$, we have

$$
\mathcal{C B}_{\mathcal{T}_{\triangleright}}\left(\mathcal{B}\left(L^{2}(\mathbb{G})\right)\right) \subseteq \mathcal{C B}_{L^{\infty}(\widetilde{\mathbb{G}})}^{L^{\infty}(\mathbb{B}}\left(\mathcal{B}\left(L_{2}(\mathbb{G})\right)\right)
$$

Proof. Let $\Phi \in \mathcal{C B}_{\mathcal{T}_{\triangleright}}\left(\mathcal{B}\left(L^{2}(\mathbb{G})\right)\right)$, and $\hat{x}, \hat{y} \in L^{\infty}(\widehat{\mathbb{G}})$. Then for any $\rho \in \mathcal{T}\left(L^{2}(\mathbb{G})\right)$ and $T \in \mathcal{B}\left(L^{2}(\mathbb{G})\right)$ we have

$$
\begin{aligned}
(\hat{x} T \hat{y}) \triangleright \rho & =(\rho \otimes \mathrm{id}) V(\hat{x} T \hat{y} \otimes 1) V^{*}=(\rho \otimes \mathrm{id})\left((\hat{x} \otimes 1) V(T \otimes 1) V^{*}(\hat{y} \otimes 1)\right) \\
& =(\hat{y} \cdot \rho \cdot \hat{x} \otimes \mathrm{id}) V(T \otimes 1) V^{*}=T \triangleright(\hat{y} \cdot \rho \cdot \hat{x}) .
\end{aligned}
$$

Thus, for any $\omega \in \mathcal{T}\left(L^{2}(\mathbb{G})\right)$ we obtain

$$
\begin{aligned}
\langle\Phi(\hat{x} T \hat{y}), \rho \triangleright \omega\rangle & =\langle\Phi(\hat{x} T \hat{y}) \triangleright \rho, \omega\rangle=\langle\Phi((\hat{x} T \hat{y}) \triangleright \rho), \omega\rangle \\
& =\langle\Phi(T \triangleright(\hat{y} \cdot \rho \cdot \hat{x})), \omega\rangle=\langle\Phi(T) \triangleright(\hat{y} \cdot \rho \cdot \hat{x}), \omega\rangle \\
& =\langle(\hat{x} \Phi(T) \hat{y}) \triangleright \rho, \omega\rangle=\langle\hat{x} \Phi(T) \hat{y}, \rho \triangleright \omega\rangle .
\end{aligned}
$$

Again by (4.1), it follows that $\Phi$ is an $L^{\infty}(\widehat{\mathbb{G}})$-bimodule map on $\mathcal{B}\left(L^{2}(\mathbb{G})\right)$.

By Proposition 4.1.6, we have $\Phi \in \in_{\bar{\nabla}} \mathcal{C B}\left(\mathcal{B}\left(L^{2}(\mathbb{G})\right)\right)$, and since $\widehat{V} \in L^{\infty}(\mathbb{G})^{\prime} \bar{\otimes} L^{\infty}(\widehat{\mathbb{G}})$, for any $x \in L^{\infty}(\mathbb{G})$ we have

$$
(\mathrm{id} \otimes \rho) \widehat{V}(\Phi(x) \otimes 1) \widehat{V}^{*}=\rho \widehat{\triangleright} \Phi(x)=\Phi(\rho \widehat{\triangleright} x)=\langle\rho, 1\rangle \Phi(x)=(\mathrm{id} \otimes \rho)(\Phi(x) \otimes 1) .
$$

It follows that $\widehat{V}(\Phi(x) \otimes 1) \widehat{V}^{*}=\Phi(x) \otimes 1$, which implies that $\widehat{\rho}(\hat{f}) \Phi(x)=\Phi(x) \widehat{\rho}(\hat{f})$ for every $\hat{f} \in L^{1}(\widehat{\mathbb{G}})$. Since $\widehat{\rho}\left(L^{1}(\widehat{\mathbb{G}})\right)$ is weak* dense in $L^{\infty}(\mathbb{G})^{\prime}$, we have $\Phi(x) \in$ $L^{\infty}(\mathbb{G})^{\prime \prime}=L^{\infty}(\mathbb{G})$. Thus, $\Phi$ leaves $L^{\infty}(\mathbb{G})$ globally invariant, and the claim follows. 


\subsection{Covariant Conditional Expectations}

Definition 4.2.1. For a locally compact quantum group $\mathbb{G}$, we say that a mapping $\Phi \in \mathcal{C B}\left(\mathcal{B}\left(L^{2}(\mathbb{G})\right)\right)$ is covariant if $\Phi \in \mathcal{C B}_{\mathcal{T}_{\triangleright}}\left(\mathcal{B}\left(L^{2}(\mathbb{G})\right)\right)$.

We now use the representation $(4.10)$ of $\operatorname{LUC}(\mathbb{G})^{*}$ to establish a one-to-one correspondence between left invariant means on $\operatorname{LUC}(\mathbb{G})$ and covariant conditional expectations onto $L^{\infty}(\widehat{\mathbb{G}})$.

Theorem 4.2.2. Let $\mathbb{G}$ be a locally compact quantum group. The following statements are equivalent:

1. $\mathbb{G}$ is amenable;

2. there is a left invariant mean on $\operatorname{LUC}(\mathbb{G})$;

3. there is a covariant conditional expectation $E: \mathcal{B}\left(L^{2}(\mathbb{G})\right) \rightarrow L^{\infty}(\widehat{\mathbb{G}})$.

Proof. (1) $\Rightarrow(2)$ : Restriction of a left invariant mean on $L^{\infty}(\mathbb{G})$ yields (2).

$(2) \Rightarrow(3)$ : Let $m \in \operatorname{LUC}(\mathbb{G})^{*}$ be a left invariant mean. Then $m \square y=\langle m, y\rangle 1$ for all $y \in \operatorname{LUC}(\mathbb{G})$ by left invariance, which gives

$$
\langle m \square m, y\rangle=\langle m, m \square y\rangle=\langle m, y\rangle\langle m, 1\rangle=\langle m, y\rangle .
$$

Hence, $m$ is a norm one idempotent in $\operatorname{LUC}(\mathbb{G})^{*}$, making $\Theta^{r}(m)$ a projection of norm one in $\mathcal{C B}_{\mathcal{T}_{\triangleright}}\left(\mathcal{B}\left(L^{2}(\mathbb{G})\right)\right)$. As such, its image is equal to its fixed points, denoted $\mathcal{H}_{\Theta(m)}$. First observe that $L^{\infty}(\widehat{\mathbb{G}}) \subseteq \mathcal{H}_{\Theta(m)}$ as $\Theta^{r}(m)(\hat{x})=(\mathrm{id} \otimes m) V(\hat{x} \otimes 1) V^{*}=\hat{x}$, $\hat{x} \in L^{\infty}(\widehat{\mathbb{G}})$. On the other hand, as $\Theta^{r}(m)$ is a $\mathcal{T}\left(L^{2}(\mathbb{G})\right)$-module map, its fixed points form a $\mathcal{T}\left(L^{2}(\mathbb{G})\right)$-submodule of $\mathcal{B}\left(L^{2}(\mathbb{G})\right)$. Thus, $T \triangleright \omega \in \mathcal{H}_{\Theta(m)}$ for every $T \in \mathcal{H}_{\Theta(m)}$ and $\omega \in \mathcal{T}\left(L^{2}(\mathbb{G})\right)$. But if $y \in \mathcal{H}_{\Theta(m)} \cap \operatorname{LUC}(\mathbb{G})$, then $x=\Theta^{r}(m)(x)=m \square x=$ $\langle m, x\rangle 1$. Hence, if $T \in \mathcal{H}_{\Theta(m)}$ and $\omega \in \mathcal{T}\left(L^{2}(\mathbb{G})\right)$ then $T \triangleright \omega=\langle m, T \triangleright \omega\rangle 1$, so that 
for any $\tau \in \mathcal{T}\left(L^{2}(\mathbb{G})\right)$

$$
\begin{aligned}
\left\langle\Gamma^{r}(T), \omega \otimes \tau\right\rangle & =\langle T, \omega \triangleright \tau\rangle=\langle T \triangleright \omega, \tau\rangle=\langle m, T \triangleright \omega\rangle\langle 1, \tau\rangle \\
& =\left\langle\Theta^{r}(m)(T), \omega\right\rangle\langle 1, \tau\rangle=\langle T \otimes 1, \omega \otimes \tau\rangle .
\end{aligned}
$$

As $\omega, \tau \in \mathcal{T}\left(L^{2}(\mathbb{G})\right)$ were arbitrary, it follows that $\Gamma^{r}(T)=V(T \otimes 1) V^{*}=T \otimes 1$, that is, $V(T \otimes 1)=(T \otimes 1) V$. Hence, $\rho(f) T=T \rho(f)$, for all $f \in L^{1}(\mathbb{G})$ and $T \in \rho\left(L^{1}(\mathbb{G})\right)^{\prime}=L^{\infty}(\widehat{\mathbb{G}})$, making $E:=\Theta^{r}(m)$ the required projection.

$(3) \Rightarrow(1)$ : If $E: \mathcal{B}\left(L^{2}(\mathbb{G})\right) \rightarrow L^{\infty}(\widehat{\mathbb{G}})$ is a conditional expectation in $\mathcal{C} \mathcal{B}_{\mathcal{T}_{\triangleright}}\left(\mathcal{B}\left(L^{2}(\mathbb{G})\right)\right)$, then $E(\operatorname{LUC}(\mathbb{G})) \subseteq \operatorname{LUC}(\mathbb{G}) \cap L^{\infty}(\widehat{\mathbb{G}})=\mathbb{C} 1$. Thus, by restriction we obtain a bounded linear functional $n \in \operatorname{LUC}(\mathbb{G})^{*}$ satisfying $\langle n, x\rangle 1=E(x)$ for all $x \in \operatorname{LUC}(\mathbb{G})$. Moreover, considering the associated map $\Theta^{r}(n) \in \mathcal{C B}_{\mathcal{T}_{\triangleright}}\left(\mathcal{B}\left(L^{2}(\mathbb{G})\right)\right)$, we see that

$$
\langle E(T), \omega\rangle 1=E(T) \triangleright \omega=E(T \triangleright \omega)=\langle n, T \triangleright \omega\rangle 1=\left\langle\Theta^{r}(n)(T), \omega\right\rangle 1
$$

for all $T \in \mathcal{B}\left(L^{2}(\mathbb{G})\right)$ and $\omega \in \mathcal{T}\left(L^{2}(\mathbb{G})\right)$. This ensures that $E=\Theta^{r}(n)$, so $E$ leaves $L^{\infty}(\mathbb{G})$ globally invariant by Corollary 4.1 .7 , and we have $E\left(L^{\infty}(\mathbb{G})\right) \subseteq L^{\infty}(\mathbb{G}) \cap$ $L^{\infty}(\widehat{\mathbb{G}})=\mathbb{C} 1$ by Proposition 3.3.5. Put $m:=\left.E\right|_{L^{\infty}(\mathbb{G})}$. Then $m \in L^{\infty}(\mathbb{G})^{*}$ is a state satisfying

$$
\langle m, x \star f\rangle 1=E(x \star f)=E\left(x \triangleright \rho_{f}\right)=E(x) \triangleright \rho_{f}=\langle m, x\rangle\langle 1, f\rangle 1
$$

for every $x \in L^{\infty}(\mathbb{G})$ and $f \in L^{1}(\mathbb{G})$, where $\pi\left(\rho_{f}\right)=f$. Hence, $m$ is a left invariant mean on $L^{\infty}(\mathbb{G})$.

Corollary 4.2.3. A locally compact group $G$ is amenable if and only if there is a covariant conditional expectation $E: \mathcal{B}\left(L^{2}(G)\right) \rightarrow \mathcal{L}(G)$.

There is a corresponding result involving right invariant means on $\operatorname{RUC}(\mathbb{G})$ and conditional expectations in $\mathcal{T}_{\triangleleft} \mathcal{C B}\left(\mathcal{B}\left(L^{2}(\mathbb{G})\right)\right.$ ). We state the result for completeness and for later use, but omit the details of the proof as the argument can easily be 
adapted from above using the left representation (4.11).

Theorem 4.2.4. Let $\mathbb{G}$ be a locally compact quantum group. The following statements are equivalent:

1. $\mathbb{G}$ is amenable;

2. there is a right invariant mean on $\mathrm{RUC}(\mathbb{G})$;

3. there is a conditional expectation $E: \mathcal{B}\left(L^{2}(\mathbb{G})\right) \rightarrow L^{\infty}\left(\widehat{\mathbb{G}}^{\prime}\right)$ in $\mathcal{T}_{\triangleleft} \mathcal{C B}\left(\mathcal{B}\left(L^{2}(\mathbb{G})\right)\right)$.

As $\mathbb{G}$ is compact if and only if it admits a left invariant mean in $L^{1}(\mathbb{G})[6$, Proposition 3.1], and the maps $\Theta^{r}(f), \Theta^{l}(f) \in \mathcal{C B}\left(\mathcal{B}\left(L^{2}(\mathbb{G})\right)\right)$ are normal for all $f \in L^{1}(\mathbb{G})$ $[53, \S 4]$, the following corollary is immediate.

Corollary 4.2.5. Let $\mathbb{G}$ be a locally compact quantum group. Then $\mathbb{G}$ is compact if and only if there is a normal covariant conditional expectation $E: \mathcal{B}\left(L^{2}(\mathbb{G})\right) \rightarrow$ $L^{\infty}(\widehat{\mathbb{G}})$.

Remark 4.2.6. In [6], a notion of topological amenability for locally compact quantum groups $\mathbb{G}$ was defined by the existence of a left invariant mean on $M\left(C_{0}(\mathbb{G})\right)$. The authors then asked if this notion of amenability is equivalent to the original one. The answer was recently provided, in the affirmative, by Zobeidi [120], generalizing the partial result of Runde in the co-amenable setting [93, Theorem 3.6]. As we always have $\operatorname{LUC}(\mathbb{G}) \subseteq M\left(C_{0}(\mathbb{G})\right.$ ), Theorem 4.2.2 provides an alternative proof (which had been found independently from [120]) for arbitrary locally compact quantum groups.

For amenable locally compact groups $G$, one may ask if every conditional expectation $E: \mathcal{B}\left(L^{2}(G)\right) \rightarrow \mathcal{L}(G)$ is covariant. As we will show in the next theorem, this is not the case.

Theorem 4.2.7. There exists a discrete amenable group $G$ and a conditional expectation $E: \mathcal{B}\left(L^{2}(G)\right) \rightarrow \mathcal{L}(G)$ that is not covariant. 
Proof. Consider the group $G=S_{\mathbb{N}}^{F}$ of finite permutations of the natural numbers. As a countable amenable discrete ICC group, its right von Neumann algebra $\mathcal{R}(G)$ is an injective $I I_{1}$-factor with separable predual [86, Proposition 7.3.20]. By [36, Proposition 3.7], its unitary group $U(\mathcal{R}(G))$ with the strong operator topology is extremely amenable, meaning there exists a multiplicative left invariant mean $m$ on $\operatorname{LUC}(U(\mathcal{R}(G)))$, the $C^{*}$-algebra of left uniformly continuous functions on the nonlocally compact group $U(\mathcal{R}(G))$. In other words, $m: \operatorname{LUC}(U(\mathcal{R}(G))) \rightarrow \mathbb{C}$ is a unital *-homomorphism satisfying

$$
\langle m, f \cdot u\rangle=\langle m, f\rangle, \quad u \in U(\mathcal{R}(G)), f \in \operatorname{LUC}(U(\mathcal{R}(G)))
$$

where $f \cdot u(v)=f(u v)$, for $u, v \in U(\mathcal{R}(G))$. Appealing to a construction of de la Harpe [25] (see also [81]), this invariant mean gives rise to a conditional expectation $E(m): \mathcal{B}\left(L^{2}(G)\right) \rightarrow \mathcal{L}(G)$ via

$$
\langle E(m)(T) \xi, \eta\rangle=\left\langle m, f_{\xi, \eta}^{T}\right\rangle, \quad T \in \mathcal{B}\left(L^{2}(G)\right), \xi, \eta \in L^{2}(G)
$$

where $f_{\xi, \eta}^{T} \in \operatorname{LUC}(U(\mathcal{R}(G)))$ is defined by $f_{\xi, \eta}^{T}(u)=\left\langle u T u^{*} \xi, \eta\right\rangle, u \in U(\mathcal{R}(G))$. Denoting the corresponding map $\operatorname{LUC}(U(\mathcal{R}(G)))^{*} \rightarrow \mathcal{C B}\left(\mathcal{B}\left(L^{2}(G)\right)\right)$ by $E$, it follows that $E$ is an isometry on states. Indeed, for any $n \in \mathbb{N}, S_{1}, T_{1}, \ldots, S_{n}, T_{n} \in \mathcal{B}\left(L^{2}(G)\right)$ and $\xi \in L^{2}(G)$,

$$
\sum_{i, j=1}^{n} f_{T_{j} \xi T_{i} \xi}^{S_{i}^{*} S_{j}}(u)=\sum_{i, j=1}^{n}\left\langle u S_{i}^{*} S_{j} u^{*} T_{j} \xi, T_{i} \xi\right\rangle=\left\langle\left(\sum_{i=1}^{n} S_{i} u^{*} T_{i}\right)^{*}\left(\sum_{j=1}^{n} S_{j} u^{*} T_{j}\right) \xi, \xi\right\rangle \geq 0
$$

for all $u \in U(\mathcal{R}(G))$. If $n \in \operatorname{LUC}(U(\mathcal{R}(G)))^{*}$ is a state then

$$
\sum_{i, j=1}^{n}\left\langle T_{i}^{*} E(n)\left(S_{i}^{*} S_{j}\right) T_{j} \xi, \xi\right\rangle=\sum_{i, j=1}^{n}\left\langle n, f_{T_{j} \xi, T_{i} \xi}^{S_{i}^{*} S_{j}}\right\rangle \geq 0
$$


so $E(n)$ is completely positive by Proposition 2.4 .3 and we have

$$
\|E(n)\|_{c b}=\|E(n)(1)\|=\|1\|=1=\|n\| .
$$

Now, suppose that $E(m)$ is covariant. By the proof of Theorem 4.2.2 there would exist a left invariant mean $n \in \operatorname{LUC}(G)^{*}$ such that $E(m)=\Theta^{r}(n)$. Viewing $G$ as a subgroup of $U(\mathcal{R}(G))$ via the right regular representation $\rho$, it follows that the restriction map $r: \operatorname{LUC}(U(\mathcal{R}(G))) \rightarrow \operatorname{LUC}(G)$ is a surjective homomorphism. Indeed, we know that $\operatorname{LUC}(G)=\mathcal{B}\left(L^{2}(G)\right) \triangleright_{a} \mathcal{T}\left(L^{2}(G)\right)$ by equation (4.9), and for any $T \in \mathcal{B}\left(L^{2}(G)\right)$ and $\omega \in \mathcal{T}\left(L^{2}(G)\right)$, we have

$$
T \triangleright_{a} \omega(s)=\left\langle\rho(s) T \rho(s)^{*}, \omega\right\rangle=r\left(f_{\omega}^{T}\right)(\rho(s)), \quad s \in G,
$$

where $f_{\omega}^{T} \in \operatorname{LUC}(U(\mathcal{R}(G)))$ is given as above by $f_{\omega}^{T}(u)=\left\langle u T u^{*}, \omega\right\rangle, u \in U(\mathcal{R}(G))$. Then

$$
\langle E(m)(T) \xi, \eta\rangle=\left\langle\Theta^{r}(n)(T) \xi, \eta\right\rangle=\left\langle n, T \triangleright \omega_{\xi, \eta}\right\rangle=\left\langle n, r\left(f_{\xi, \eta}^{T}\right)\right\rangle=\left\langle E\left(r^{*}(n)\right)(T) \xi, \eta\right\rangle
$$

for all $T \in \mathcal{B}\left(L^{2}(G)\right)$ and $\xi, \eta \in L^{2}(G)$, so $r^{*}(n)=m$ as $E$ is injective on states. Thus, given $f, g \in \operatorname{LUC}(G)$, take $\widetilde{f}, \widetilde{g} \in \operatorname{LUC}(U(\mathcal{R}(G)))$ satisfying $r(\widetilde{f})=f$ and $r(\widetilde{g})=g$. Then

$$
\langle n, f g\rangle=\langle n, r(\widetilde{f}) r(\widetilde{g})\rangle=\langle n, r(\widetilde{f} \widetilde{g})\rangle=\langle m, \widetilde{f} \widetilde{g}\rangle=\langle m, \widetilde{f}\rangle\langle m, \widetilde{g}\rangle=\langle n, f\rangle\langle n, g\rangle .
$$

Hence, $n$ is a multiplicative left invariant mean on $\operatorname{LUC}(G)$, which makes $G=\{e\}$ by $[38$, Lemma 4] - a contradiction.

Remark 4.2.8. In [25], de la Harpe showed that a von Neumann algebra $M \subseteq \mathcal{B}(H)$ is injective if and only if there is a left invariant mean on $\operatorname{LUC}\left(U\left(M^{\prime}\right)\right)$, where $U\left(M^{\prime}\right)$ denotes the unitary group of $M^{\prime}$ under the strong operator topology. For any such invariant mean $m$, the map $E(m)$ defined in Theorem 4.2.7 is a conditional expectation 
from $\mathcal{B}(H)$ onto $M$. The natural question then arises: is every conditional expectation $E: \mathcal{B}(H) \rightarrow M$ of the form $E(m)$ for some invariant mean $m$ on $\operatorname{LUC}\left(U\left(M^{\prime}\right)\right)$ ? Moreover, it would be interesting to find a characterization of conditional expectations $\mathcal{B}(H) \rightarrow M$ arising from extreme invariant means.

In Theorem 4.2.2, we characterized the amenability of $\mathbb{G}$ by means of a conditional expectation $E: \mathcal{B}\left(L^{2}(\mathbb{G})\right) \rightarrow L^{\infty}(\widehat{\mathbb{G}})$ commuting with the right $\left(\mathcal{T}\left(L^{2}(\mathbb{G})\right), \triangleright\right)$ module action on $\mathcal{B}\left(L^{2}(\mathbb{G})\right.$ ). As there are seven other $\mathcal{T}\left(L^{2}(\mathbb{G})\right.$ )-module structures on $\mathcal{B}\left(L^{2}(\mathbb{G})\right)$, we will now characterize the existence of module projections $E$ : $\mathcal{B}\left(L^{2}(\mathbb{G})\right) \rightarrow L^{\infty}(\widehat{\mathbb{G}})$ in each of the remaining cases. To this end, we denote by $\mathcal{T}_{\triangleright} \mathcal{C B}\left(\mathcal{B}\left(L^{2}(\mathbb{G})\right)\right)\left(\right.$ respectively, $\left.\mathcal{C B}_{\mathcal{T}_{\triangleleft}}\left(\mathcal{B}\left(L^{2}(\mathbb{G})\right)\right)\right)$ the space of completely bounded left $\left(\mathcal{T}\left(L^{2}(\mathbb{G})\right), \triangleright\right)$-module (respectively, right $\left(\mathcal{T}\left(L^{2}(\mathbb{G})\right), \triangleleft\right)$-module) maps on $\mathcal{B}\left(L^{2}(\mathbb{G})\right)$, and similarly for the dual products $\widehat{\triangleright}$ and $\widehat{\triangleleft}$.

Proposition 4.2.9. Let $\mathbb{G}$ be a locally compact quantum group. There exists a conditional expectation $E: \mathcal{B}\left(L^{2}(\mathbb{G})\right) \rightarrow L^{\infty}(\widehat{\mathbb{G}})$ in ${ }_{\mathcal{T}_{\triangleright}} \mathcal{C B}\left(\mathcal{B}\left(L^{2}(\mathbb{G})\right)\right)$ if and only if $\mathbb{G}$ is amenable.

Proof. By restriction, we may view any $f \in L^{1}(\mathbb{G}) \subseteq L^{\infty}(\mathbb{G})^{*}$ as an element of LUC $(\mathbb{G})^{*}$. Moreover, if $\pi:\left(\mathcal{T}\left(L^{2}(\mathbb{G})\right), \triangleright\right) \rightarrow\left(L^{1}(\mathbb{G}), \star\right)$ denotes the restriction map (4.2), for $\omega, \rho \in \mathcal{T}\left(L^{2}(\mathbb{G})\right)$ and $T \in \mathcal{B}\left(L^{2}(\mathbb{G})\right)$ we have

$$
\langle\rho \triangleright T, \omega\rangle=\langle T, \omega \triangleright \rho\rangle=\langle T \triangleright \omega, \rho\rangle=\langle T \triangleright \omega, \pi(\rho)\rangle=\left\langle\Theta^{r}(\pi(\rho))(T), \omega\right\rangle .
$$

Thus, $\rho \triangleright T=\Theta^{r}(\pi(\rho))(T)$, so that a map $\Phi \in \mathcal{C B}\left(\mathcal{B}\left(L^{2}(\mathbb{G})\right)\right)$ is a left $\left(\mathcal{T}\left(L^{2}(\mathbb{G})\right)\right.$, $)$ module homomorphism if and only if $\Phi \in \Theta^{r}\left(L^{1}(\mathbb{G})\right)^{c}$.

If $\mathbb{G}$ is amenable, then there exists a two-sided invariant mean $m$ on $L^{\infty}(\mathbb{G})$. Denoting again by $m$ its restriction to $\operatorname{LUC}(\mathbb{G})$, it follows that

$$
m \square f=f \square m=\langle f, 1\rangle m
$$


for every $f \in L^{1}(\mathbb{G})$. Hence, $\Theta^{r}(m) \in \Theta^{r}\left(L^{1}(\mathbb{G})\right)^{c}$ by (4.10). Moreover, the proof of Theorem 4.2.2 implies that $\Theta^{r}(m)$ is a conditional expectation onto $L^{\infty}(\widehat{\mathbb{G}})$.

Conversely, suppose that there exists a conditional expectation $E: \mathcal{B}\left(L^{2}(\mathbb{G})\right) \rightarrow$ $L^{\infty}(\widehat{\mathbb{G}})$ in $\mathcal{T}_{\triangleright} \mathcal{C B}\left(\mathcal{B}\left(L^{2}(\mathbb{G})\right)\right)$, and let $\hat{f} \in L^{1}(\widehat{\mathbb{G}})$ be a state. For $\omega \in \mathcal{T}\left(L^{2}(\mathbb{G})\right)$ with $f=\pi(\omega) \in L^{1}(\mathbb{G})$ and $x \in L^{\infty}(\mathbb{G})$, the relations (4.6) imply

$$
\langle\hat{f} \circ E, f \star x\rangle=\langle\hat{f} \circ E, \omega \triangleright x\rangle=\langle\hat{f}, \omega \triangleright E(x)\rangle=\langle\omega, 1\rangle\langle\hat{f} \circ E, x\rangle=\langle f, 1\rangle\langle\hat{f} \circ E, x\rangle .
$$

Thus, $\hat{f} \circ E$ is a right invariant mean on $L^{\infty}(\mathbb{G})$.

Proposition 4.2.10. Let $\mathbb{G}$ be a locally compact quantum group. There exists a conditional expectation $E: \mathcal{B}\left(L^{2}(\mathbb{G})\right) \rightarrow L^{\infty}(\widehat{\mathbb{G}})$ in $\mathcal{C B}_{\mathcal{T}_{\triangleleft}}\left(\mathcal{B}\left(L^{2}(\mathbb{G})\right)\right.$ if and only if $L^{\infty}(\widehat{\mathbb{G}})$ is injective.

Proof. Suppose that $\widehat{\mathbb{G}}$ is injective. Then there exists a conditional expectation $E$ : $\mathcal{B}\left(L^{2}(\mathbb{G})\right) \rightarrow L^{\infty}(\widehat{\mathbb{G}})$. By [105], $E$ is an $L^{\infty}(\widehat{\mathbb{G}})$-bimodule map on $\mathcal{B}\left(L^{2}(\mathbb{G})\right)$. We will show that it also lies in $\mathcal{C B}_{\mathcal{T}_{\triangleleft}}\left(\mathcal{B}\left(L^{2}(\mathbb{G})\right)\right)$. To this end, observe that a map $\Phi \in \mathcal{C B}\left(\mathcal{B}\left(L^{2}(\mathbb{G})\right)\right)$ is a right $\left(\mathcal{T}\left(L^{2}(\mathbb{G})\right), \triangleleft\right)$-module map if and only if $\Phi \in \Theta^{l}\left(L^{1}(\mathbb{G})\right)^{c}$ (see the proof of Proposition 4.2.9). If $f \in L^{1}(\mathbb{G})$, then from [53, Theorem 4.10], $\Theta^{l}(f)$ is a normal completely bounded $L^{\infty}\left(\widehat{\mathbb{G}}^{\prime}\right)$-bimodule map on $\mathcal{B}\left(L^{2}(\mathbb{G})\right)$, which by [41] implies the existence of two nets $\left(\hat{a}_{i}\right)_{i \in I}$ and $\left(\hat{b}_{i}\right)_{i \in I}$ in $L^{\infty}(\widehat{\mathbb{G}})$ such that

$$
\Theta^{l}(f)(T)=\sum_{i \in I} \hat{a}_{i} T \hat{b}_{i}
$$

where the sum converges in the weak ${ }^{*}$ topology of $\mathcal{B}\left(L^{2}(\mathbb{G})\right)$ for all $T \in \mathcal{B}\left(L^{2}(\mathbb{G})\right)$. By [71, Lemma 2.3] we may approximate $E$ in the weak* topology of $\mathcal{C B}\left(\mathcal{B}\left(L^{2}(\mathbb{G})\right)\right)$ by a net of normal completely bounded $L^{\infty}(\widehat{\mathbb{G}})$-bimodule maps $\left(\Phi_{j}\right)_{j \in J}$. Consequently, 
for $T \in \mathcal{B}\left(L^{2}(\mathbb{G})\right)$ and $\rho \in \mathcal{T}\left(L^{2}(\mathbb{G})\right)$,

$$
\begin{aligned}
\left\langle E\left(\Theta^{l}(f)(T)\right), \rho\right\rangle & =\lim _{j \in J}\left\langle\Phi_{j}\left(\Theta^{l}(f)(T)\right), \rho\right\rangle=\lim _{j \in J} \sum_{i \in I}\left\langle\Phi_{j}\left(\hat{a}_{i} T \hat{b}_{i}\right), \rho\right\rangle \\
& =\lim _{j \in J} \sum_{i \in I}\left\langle\hat{a}_{i} \Phi_{j}(T) \hat{b}_{i}, \rho\right\rangle=\lim _{j \in J}\left\langle\Theta^{l}(f)\left(\Phi_{j}(T)\right), \rho\right\rangle \\
& =\left\langle\Theta^{l}(f)(E(T)), \rho\right\rangle .
\end{aligned}
$$

Since $f \in L^{1}(\mathbb{G})$ was arbitrary, we have $E \in \Theta^{l}\left(L^{1}(\mathbb{G})\right)^{c}$. The converse is trivial.

Remark 4.2.11. After the above proposition was proven, it came to the authors' attention that Soltan and Viselter had independently obtained a related result in [95]; the main result of [95] also characterizes amenability of $\mathbb{G}$ in terms of the existence of certain conditional expectations (namely those mapping $L^{\infty}(\mathbb{G})$ into the center of $\left.L^{\infty}(\widehat{\mathbb{G}})\right)$, however, the authors do not adopt a homological viewpoint.

Corollary 4.2.12. Let $\mathbb{G}$ be a locally compact quantum group for which there exists a state $M \in \mathcal{B}\left(L^{2}(\mathbb{G})\right)^{*}$ satisfying $M(\rho \triangleright T)=M(T \triangleleft \rho)$ for all $T \in \mathcal{B}\left(L^{2}(\mathbb{G})\right)$ and $\rho \in \mathcal{T}\left(L^{2}(\mathbb{G})\right)$. Then $\mathbb{G}$ is amenable if and only if $L^{\infty}(\widehat{\mathbb{G}})$ is injective.

Proof. Suppose $E: \mathcal{B}\left(L^{2}(\mathbb{G})\right) \rightarrow L^{\infty}(\widehat{\mathbb{G}})$ is a conditional expectation. Then by Proposition 4.2.10, $E$ is a right $\left(\mathcal{T}\left(L^{2}(\mathbb{G})\right), \triangleleft\right)$-module map. Thus, $N:=M \circ E$ is a state on $\mathcal{B}\left(L^{2}(\mathbb{G})\right)$ satisfying $N(T \triangleleft \rho)=M(E(T) \triangleleft \rho)=M(\rho \triangleright E(T))=\langle\rho, 1\rangle N(T)$ for all $T \in \mathcal{B}\left(L^{2}(\mathbb{G})\right)$ and $\rho \in \mathcal{T}\left(L^{2}(\mathbb{G})\right)$. It follows that $\left.N\right|_{L^{\infty}(\mathbb{G})}$ is a left invariant mean on $L^{\infty}(\mathbb{G})$.

Remark 4.2.13. The above condition, i.e., the existence of a state $M \in \mathcal{B}\left(L^{2}(\mathbb{G})\right)^{*}$ such that $M(\rho \triangleright T)=M(T \triangleleft \rho)$ for all $T \in \mathcal{B}\left(L^{2}(\mathbb{G})\right)$ and $\rho \in \mathcal{T}\left(L^{2}(\mathbb{G})\right)$, may be seen as a form of inner amenability for locally compact quantum groups. We will systematically pursue this notion in the final chapter.

Proposition 4.2.14. Let $\mathbb{G}$ be a locally compact quantum group. There exists a conditional expectation $E: \mathcal{B}\left(L^{2}(\mathbb{G})\right) \rightarrow L^{\infty}(\widehat{\mathbb{G}})$ in ${ }_{\mathcal{T}_{\triangleleft}} \mathcal{C B}\left(\mathcal{B}\left(L^{2}(\mathbb{G})\right)\right)$ if and only if $\mathbb{G}$ is co-commutative, i.e., $L^{\infty}(\mathbb{G})=\mathcal{L}(G)$ for some locally compact group $G$. 
Proof. If $\mathbb{G}$ is co-commutative, then $L^{\infty}(\mathbb{G})=\mathcal{L}(G)$ for some locally compact group $G$, and by $[85$, Theorem 4] there exists a right invariant mean $m$ on $\mathcal{L}(G)$. In this case, its restriction to $\operatorname{UCB}(\widehat{G})=\operatorname{RUC}(\mathbb{G})$ is also a right invariant mean, and Theorem 4.2.4 provides a conditional expectation $\Theta^{l}(m): \mathcal{B}\left(L^{2}(\mathbb{G})\right) \rightarrow L^{\infty}\left(\widehat{\mathbb{G}}^{\prime}\right)$ in $\mathcal{T}_{\triangleleft} \mathcal{C B}\left(\mathcal{B}\left(L^{2}(\mathbb{G})\right)\right)$. By duality, $L^{\infty}(\widehat{\mathbb{G}})=L^{\infty}(G)=L^{\infty}\left(\widehat{\mathbb{G}}^{\prime}\right)$, making $\Theta^{l}(m)$ the desired projection.

If $E: \mathcal{B}\left(L^{2}(\mathbb{G})\right) \rightarrow L^{\infty}(\widehat{\mathbb{G}})$ exists in $\mathcal{T}_{\triangleleft} \mathcal{C B}\left(\mathcal{B}\left(L^{2}(\mathbb{G})\right)\right.$ ), then a simple calculation implies that $(E \otimes \mathrm{id}) \circ \Gamma^{l}=\Gamma^{l} \circ E . \quad$ As $\Gamma^{l}(\cdot)=W^{*}(1 \otimes(\cdot)) W$, with $W \in$ $L^{\infty}(\mathbb{G}) \bar{\otimes} L^{\infty}(\widehat{\mathbb{G}})$, and $E\left(\mathcal{B}\left(L^{2}(\mathbb{G})\right)\right)=L^{\infty}(\widehat{\mathbb{G}})$, we must have $(E \otimes \mathrm{id}) \circ \Gamma^{l}(T)=$ $\Gamma^{l} \circ E(T) \in L^{\infty}(\mathbb{G}) \bar{\otimes} L^{\infty}(\widehat{\mathbb{G}})$ for every $T \in \mathcal{B}\left(L^{2}(\mathbb{G})\right)$. In particular, for $\hat{x}^{\prime} \in L^{\infty}\left(\widehat{\mathbb{G}}^{\prime}\right)$, we have

$$
(E \otimes \mathrm{id}) \circ \Gamma^{l}\left(\hat{x}^{\prime}\right)=(E \otimes \mathrm{id})\left(W^{*}\left(1 \otimes \hat{x}^{\prime}\right) W\right)=1 \otimes \hat{x}^{\prime} \in L^{\infty}(\mathbb{G}) \bar{\otimes} L^{\infty}(\widehat{\mathbb{G}}),
$$

implying that $L^{\infty}\left(\widehat{\mathbb{G}}^{\prime}\right) \subseteq L^{\infty}(\widehat{\mathbb{G}})$. As $L^{\infty}(\widehat{\mathbb{G}})$ is in standard form on $\mathcal{B}\left(L^{2}(\mathbb{G})\right)$, there exists a conjugate linear isometric involution $\widehat{J}$ on $L^{2}(\mathbb{G})$ satisfying $\widehat{J} L^{\infty}(\widehat{\mathbb{G}}) \widehat{J}=$ $L^{\infty}\left(\widehat{\mathbb{G}}^{\prime}\right)$. We therefore obtain $L^{\infty}(\widehat{\mathbb{G}}) \subseteq L^{\infty}\left(\widehat{\mathbb{G}}^{\prime}\right)$, that is, $L^{\infty}(\widehat{\mathbb{G}})$ is commutative. By $[99,109$, Theorem $2 ; \S 2], L^{\infty}(\widehat{\mathbb{G}})=L^{\infty}(G)$ for some locally compact group $G$, making $L^{\infty}(\mathbb{G})$ co-commutative.

Proposition 4.2.15. Let $\mathbb{G}$ be a locally compact quantum group. There exists a conditional expectation $E: \mathcal{B}\left(L^{2}(\mathbb{G})\right) \rightarrow L^{\infty}(\widehat{\mathbb{G}})$ in $\mathcal{C B}_{\mathcal{T}_{\bar{\unrhd}}}\left(\mathcal{B}\left(L^{2}(\mathbb{G})\right)\right)$ if and only if $\mathbb{G}=\mathbb{C} 1$.

Proof. For any $T \in \mathcal{B}\left(L^{2}(\mathbb{G})\right)$ and $\rho \in \mathcal{T}\left(L^{2}(\mathbb{G})\right)$, we have $T \widehat{\triangleright} \rho \in L^{\infty}(\widehat{\mathbb{G}})$, so if such a conditional expectation $E$ exists, then $T \widehat{\triangleright} \rho=E(T \widehat{\triangleright} \rho)=E(T) \widehat{\triangleright} \rho$. By density of products $(4.1)$, it follows that $E(T)=T$. In particular $\mathcal{B}\left(L^{2}(\mathbb{G})\right) \subseteq L^{\infty}(\widehat{\mathbb{G}})$, which entails that $\mathbb{G}=\widehat{\mathbb{G}}=\mathbb{C} 1$. The converse is trivial.

Proposition 4.2.16. Let $\mathbb{G}$ be a locally compact quantum group. There exists a conditional expectation $E: \mathcal{B}\left(L^{2}(\mathbb{G})\right) \rightarrow L^{\infty}(\widehat{\mathbb{G}})$ in $\tau_{\tau_{\mathcal{\triangleleft}}} \mathcal{C B}\left(\mathcal{B}\left(L^{2}(\mathbb{G})\right)\right)$ if and only if $\mathbb{G}=\mathbb{C} 1$. 
Proof. Using the extended unitary antipode of $\widehat{\mathbb{G}}$, denoted by $\widehat{R}$, it follows that $\widehat{R} \circ E \circ$ $\widehat{R}$ is a conditional expectation onto $L^{\infty}(\widehat{\mathbb{G}})$ in $\mathcal{C B}_{\mathcal{T}_{\widehat{\widehat{D}}}}\left(\mathcal{B}\left(L^{2}(\mathbb{G})\right)\right)$, so the result follows from Proposition 4.2.15.

Proposition 4.2.17. Let $\mathbb{G}$ be a locally compact quantum group. There exists a conditional expectation $E: \mathcal{B}\left(L^{2}(\mathbb{G})\right) \rightarrow L^{\infty}(\widehat{\mathbb{G}})$ in $\mathcal{T}_{\bar{\S}} \mathcal{C B}\left(\mathcal{B}\left(L^{2}(\mathbb{G})\right)\right)$ if and only if $\mathbb{G}$ is amenable.

Proof. If $\mathbb{G}$ is amenable, then by Theorem 4.2.2 there exits a conditional expectation onto $L^{\infty}(\widehat{\mathbb{G}})$ in $\mathcal{C B}_{\mathcal{T}_{\triangleright}}\left(\mathcal{B}\left(L^{2}(\mathbb{G})\right)\right)$, which, thanks to Proposition 4.1.6, lies in $\mathcal{T}_{\bar{\S}} \mathcal{C B}\left(\mathcal{B}\left(L^{2}(\mathbb{G})\right)\right)$.

On the other hand, if there exists a conditional expectation $E$ in $\mathcal{T}_{\bar{\Delta}} \mathcal{C B}\left(\mathcal{B}\left(L^{2}(\mathbb{G})\right)\right)$, then for any $x \in L^{\infty}(\mathbb{G})$ and $\rho \in \mathcal{T}\left(L^{2}(\mathbb{G})\right)$ we have

$$
\rho \widehat{\triangleright} E(x)=E(\rho \widehat{\triangleright} x)=\langle\rho, 1\rangle E(x) .
$$

As in the proof of Corollary 4.1.7, this implies $E(x) \in L^{\infty}(\mathbb{G})$. But then $E(x) \in$ $L^{\infty}(\mathbb{G}) \cap L^{\infty}(\widehat{\mathbb{G}})=\mathbb{C} 1$ by Proposition 3.3.5. Define $m \in L^{\infty}(\mathbb{G})^{*}$ by $\langle m, x\rangle 1=E(x)$, $x \in L^{\infty}(\mathbb{G})$. Let $f \in L^{1}(\mathbb{G})$ with $\omega \in \mathcal{T}\left(L^{2}(\mathbb{G})\right)$ satisfying $f=\left.\omega\right|_{L^{\infty}(\mathbb{G})}$. Then since $E \in \mathcal{C B}_{\mathcal{T}_{\triangleleft}}\left(\mathcal{B}\left(L^{2}(\mathbb{G})\right)\right)$ by the proof of Proposition 4.2.10, for any $x \in L^{\infty}(\mathbb{G})$ we obtain

$$
\langle m, x \star f\rangle=\langle m, x \triangleleft \omega\rangle=E(x \triangleleft \omega)=E(x) \triangleleft \omega=\langle f, 1\rangle\langle m, x\rangle .
$$

As $f \in L^{1}(\mathbb{G})$ was arbitrary, $\mathbb{G}$ is amenable.

Proposition 4.2.18. Let $\mathbb{G}$ be a locally compact quantum group. There exists a conditional expectation $E: \mathcal{B}\left(L^{2}(\mathbb{G})\right) \rightarrow L^{\infty}(\widehat{\mathbb{G}})$ in $\mathcal{C B}_{\mathcal{T}_{\widehat{\triangleleft}}}\left(\mathcal{B}\left(L^{2}(\mathbb{G})\right)\right)$ if and only if $\mathbb{G}$ is amenable.

Proof. This follows from Proposition 4.2.17 using the extended unitary antipode $\widehat{R}$. 
We record the normal version of Proposition 4.2.18 for later use. The proof follows accordingly and is left to the reader to establish.

Proposition 4.2.19. Let $\mathbb{G}$ be a locally compact quantum group. There exists a normal conditional expectation $E: \mathcal{B}\left(L^{2}(\mathbb{G})\right) \rightarrow L^{\infty}(\widehat{\mathbb{G}})$ in $\mathcal{C B}_{\mathcal{T}_{\widehat{\triangleleft}}}\left(\mathcal{B}\left(L^{2}(\mathbb{G})\right)\right)$ if and only if $\mathbb{G}$ is compact.

Remark 4.2.20. By the proof of [90, Theorem 2.1], it follows that a locally compact quantum group $\mathbb{G}$ is amenable if and only if there exists a non-zero left (respectively, right, two-sided) invariant functional $m \in L^{\infty}(\mathbb{G})^{*}$. Hence, the existence of a completely bounded covariant projection $E: \mathcal{B}\left(L^{2}(\mathbb{G})\right) \rightarrow L^{\infty}(\widehat{\mathbb{G}})$ is equivalent to the amenability of $\mathbb{G}$, and it follows that we may replace "conditional expectation" by "completely bounded projection" in the statements of all the results in this section, except for Propositions 4.2.17, 4.2.18, and 4.2.19. Recall that a von Neumann algebra $M \subseteq \mathcal{B}(H)$ is injective if and only if there exists a completely bounded projection $E: \mathcal{B}(H) \rightarrow M[14,84]$.

\subsection{Injective Quantum Group Modules}

Continuing in the spirit of the previous section, below we establish a perfect duality between quantum group amenability and injectivity in the category of $\mathcal{T}\left(L^{2}(\mathbb{G})\right.$ )- and $L^{1}(\mathbb{G})$-modules. We also show that both amenability of $\mathbb{G}$ and of $\widehat{\mathbb{G}}$ may be characterized through the injectivity of $\mathcal{B}\left(L^{2}(\mathbb{G})\right)$ as a left, respectively, right $\mathcal{T}\left(L^{2}(\mathbb{G})\right.$ )module. We begin with a few general results on the homology of operator modules which appear to be new.

Proposition 4.3.1. Let $\mathcal{A}$ be a completely contractive Banach algebra and let $X \in$ $\bmod -\mathcal{A}$. If $X$ is $C_{1}$-projective in $\bmod -\mathbb{C}$ and is relatively $C_{2}$-projective in $\bmod -\mathcal{A}$, then $X$ is $C_{1} C_{2}$-projective in $\bmod -\mathcal{A}$.

Proof. Let $Y, Z \in \bmod -\mathcal{A}$, let $\Psi: Y \rightarrow Z$ be a complete quotient morphism and $\Phi: X \rightarrow Z$ be a morphism. By relative $C_{2}$-projectivity, there exists a morphism 
$\alpha: X \rightarrow X \widehat{\otimes} \mathcal{A}^{+}$satisfying $m_{X}^{+} \circ \alpha=\mathrm{id}_{X}$ and $\|\alpha\|_{c b} \leq C_{2}$, where $m_{X}^{+}: X \widehat{\otimes} \mathcal{A}^{+} \rightarrow$ $X$ is the extended multiplication map. Since $X$ is a $C_{1}$-projective operator space, for every $\varepsilon>0$, there exists a lifting $\Phi_{\varepsilon}: X \rightarrow Y$ satisfying $\Psi \circ \Phi_{\varepsilon}=\Phi$ and $\left\|\Phi_{\varepsilon}\right\|_{c b}<C_{1}\|\Phi\|_{c b}+\varepsilon / C_{2}$. The mapping $\left(\Phi_{\varepsilon} \otimes \mathrm{id}\right): X \widehat{\otimes} \mathcal{A}^{+} \rightarrow Y \widehat{\otimes} \mathcal{A}^{+}$is completely bounded with $\left\|\Phi_{\varepsilon} \otimes \mathrm{id}\right\|<C_{1}\|\Phi\|_{c b}+\varepsilon / C_{2}$, and composing with $\alpha$ together with the multiplication $m_{Y}^{+}: Y \widehat{\otimes} \mathcal{A}^{+} \rightarrow Y$ we obtain a morphism $\widetilde{\Phi}_{\varepsilon}=m_{Y}^{+} \circ\left(\Phi_{\varepsilon} \otimes \mathrm{id}\right) \circ \alpha$ : $X \rightarrow Y$ satisfying $\left\|\widetilde{\Phi}_{\varepsilon}\right\|<C_{1} C_{2}\|\Phi\|_{c b}+\varepsilon$. Moreover, using the module properties of the pertinent morphisms we have

$$
\begin{aligned}
\Psi \circ \widetilde{\Phi}_{\varepsilon} & =\Psi \circ m_{Y}^{+} \circ\left(\Phi_{\varepsilon} \otimes \mathrm{id}\right) \circ \alpha \\
& =m_{Z}^{+} \circ(\Psi \otimes \mathrm{id}) \circ\left(\Phi_{\varepsilon} \otimes \mathrm{id}\right) \circ \alpha \\
& =m_{Z}^{+} \circ(\Phi \otimes \mathrm{id}) \circ \alpha \\
& =\Phi \circ m_{X}^{+} \circ \alpha \\
& =\Phi .
\end{aligned}
$$

Hence, $X$ is $C_{1} C_{2}$-projective.

Example 4.3.2. The converse of Proposition 4.3.1 (when $C_{1}=C_{2}=1$ ) is not true in general as $\mathcal{A}$ is both 1-projective and relatively 1-projective in $\bmod -\mathcal{A}$ for any unital $C^{*}$-algebra. However, the only $C^{*}$-algebra which is a 1 -projective operator space is $\mathbb{C}$ by $[8$, Theorem 3.4].

Given a completely contractive Banach algebra $\mathcal{A}$ and $X \in \bmod -\mathcal{A}$, there is a canonical completely contractive morphism $\Delta^{+}: X \rightarrow \mathcal{C B}\left(\mathcal{A}^{+}, X\right)$ given by

$$
\Delta^{+}(x)(a)=x \cdot a, \quad x \in X, a \in \mathcal{A}^{+}
$$

where the right $\mathcal{A}$-module structure on $\mathcal{C B}\left(\mathcal{A}^{+}, X\right)$ is defined by

$$
(\Psi \cdot a)(b)=\Psi(a b), \quad a \in \mathcal{A}, \Psi \in \mathcal{C B}\left(\mathcal{A}^{+}, X\right), b \in \mathcal{A}^{+} .
$$


An analogous construction exists for objects in $\mathcal{A}-\bmod$ and $X \in \mathcal{A}-\bmod -\mathcal{A}$.

The following well-known characterization of relative injectivity will be used several times throughout this thesis. The proof follows verbatim from the classical result [45, Theorem VII.1.39] (see also [114, Proposition 3.8]).

Proposition 4.3.3. Let $\mathcal{A}$ be a completely contractive Banach algebra and let $X \in$ $\bmod -\mathcal{A}$. Then $X$ is relatively $C$-injective if and only if there exists a morphism $\Phi: \mathcal{C B}\left(\mathcal{A}^{+}, X\right) \rightarrow X$ with $\|\Phi\|_{c b} \leq C$ that is a left inverse to $\Delta^{+}$. Moreover, if $X$ is faithful, then $X$ is relatively $C$-injective if and only if there exists a morphism $\Phi: \mathcal{C B}(\mathcal{A}, X) \rightarrow X$ with $\|\Phi\|_{c b} \leq C$ that is a left inverse to $\Delta: X \rightarrow \mathcal{C B}(\mathcal{A}, X)$, where $\Delta(x)(a):=\Delta^{+}(x)(a)$ for all $x \in X$ and $a \in \mathcal{A}$.

In the case of operator bimodules we also have a similar result, which we state in the special case where $X=\mathcal{A}^{*} \in \mathcal{A}-\bmod -\mathcal{A}$. The proof follows from the operator space versions of [92, Lemma 5.3.10, Theorem 5.3.12], so we leave the details to the reader.

Proposition 4.3.4. Let $\mathcal{A}$ be a completely contractive Banach algebra. Then $\mathcal{A}^{*}$ is relatively $C$-injective in $\mathcal{A}-\bmod -\mathcal{A}$ if and only if there exists a morphism $\Phi:(\mathcal{A} \widehat{\otimes} \mathcal{A})^{*} \rightarrow \mathcal{A}^{*}$ satisfying $\|\Phi\|_{c b} \leq C$ and $\Phi \circ m_{\mathcal{A}}^{*}=\operatorname{id}_{\mathcal{A}^{*}}$, where $m_{\mathcal{A}}: \mathcal{A} \widehat{\otimes} \mathcal{A} \rightarrow \mathcal{A}$ is the multiplication map.

Using Proposition 4.3.3, we now prove an analogue of Proposition 4.3.1 for injective modules.

Proposition 4.3.5. Let $\mathcal{A}$ be a completely contractive Banach algebra and let $X \in$ $\bmod -\mathcal{A}$. If $X$ is $C_{1}$-injective in $\bmod -\mathbb{C}$ and is relatively $C_{2}$-injective in $\bmod -\mathcal{A}$, then $X$ is $C_{1} C_{2}$-injective in $\bmod -\mathcal{A}$.

Proof. We first show that $\mathcal{C B}\left(\mathcal{A}^{+}, X\right)$ is $C_{1}$-injective in $\bmod -\mathcal{A}$ using the standard argument. To this end, let $Y, Z \in \bmod -\mathcal{A}$, let $\kappa: Y \hookrightarrow Z$ be a completely isometric morphism, and let $\alpha: Y \rightarrow \mathcal{C B}\left(\mathcal{A}^{+}, X\right)$ be a morphism. Let $\beta: Y \rightarrow X$ 
be defined by $\beta(y)=\alpha(y)(e), y \in Y$, where $e \in \mathcal{A}^{+}$is the unit. By $C_{1}$-injectivity of $X$ in $\bmod -\mathbb{C}$, there exists an extension $\widetilde{\beta}: Z \rightarrow X$ satisfying $\beta=\widetilde{\beta} \circ \kappa$ and $\|\widetilde{\beta}\|_{c b} \leq C_{1}\|\beta\|_{c b} \leq C_{1}\|\alpha\|_{c b}$. Define $\widetilde{\alpha}: Z \rightarrow \mathcal{C B}\left(\mathcal{A}^{+}, X\right)$ by $\widetilde{\alpha}(z)(a)=\widetilde{\beta}(z \cdot a)$, for $z \in Z, a \in \mathcal{A}^{+}$. Then

$$
\widetilde{\alpha}(z) \cdot a(b)=\widetilde{\alpha}(z)(a b)=\widetilde{\beta}(z \cdot a b)=\widetilde{\alpha}(z \cdot a)(b)
$$

for all $z \in Z$ and $a, b \in \mathcal{A}$. Thus, $\widetilde{\alpha}$ is a morphism extending $\alpha$ such that $\|\widetilde{\alpha}\|_{c b} \leq$ $\|\widetilde{\beta}\|_{c b} \leq C_{1}\|\alpha\|_{c b}$.

Now, by relative $C_{2}$-injectivity of $X$ in $\bmod -\mathcal{A}$, Proposition 4.3 .3 entails the existence of a morphism $\Phi: \mathcal{C B}\left(\mathcal{A}^{+}, X\right) \rightarrow X$ satisfying $\Phi \circ \Delta^{+}=\operatorname{id}_{X}$ and $\|\Phi\|_{c b} \leq C_{2}$. Thus, if $Y, Z \in \bmod -\mathcal{A}$ with $\kappa: Y \hookrightarrow Z$ a completely isometric morphism, and $\alpha$ : $Y \rightarrow X$ is a morphism, then we may extend the morphism $\Delta^{+} \circ \alpha: Y \rightarrow \mathcal{C B}\left(\mathcal{A}^{+}, X\right)$ to a morphism $\widetilde{\alpha}: Z \rightarrow \mathcal{C B}\left(\mathcal{A}^{+}, X\right)$ with $\|\widetilde{\alpha}\|_{c b} \leq C_{1}\left\|\Delta^{+} \circ \alpha\right\|_{c b} \leq C_{1}\|\alpha\|_{c b}$. Hence, the morphism $\Phi \circ \widetilde{\alpha}: Z \rightarrow X$ is the desired extension satisfying $\|\Phi \circ \widetilde{\alpha}\|_{c b} \leq C_{1} C_{2}\|\alpha\|_{c b}$.

Example 4.3.6. The converse of Proposition 4.3.5 is not true in general (when $C_{1}=C_{2}=1$ ). Indeed, for any unital completely contractive Banach algebra $\mathcal{A}$ and any 1-injective operator space $X$, it follows from the proof of Proposition 4.3.5 that $\mathcal{C B}(\mathcal{A}, X)$ is 1 -injective in $\bmod -\mathcal{A}$. This clearly implies relative 1-injectivity in $\bmod -\mathcal{A}$. However, consider $\mathcal{A}=B(G)$ and $X=\mathbb{C}$, where $G$ is a non-amenable discrete group. Then $\mathcal{C B}(\mathcal{A}, X)=B(G)^{*}=C^{*}(G)^{* *}$, and if this were a 1 -injective operator space, the group $C^{*}$-algebra $C^{*}(G)$ would be nuclear, forcing $G$ to be amenable by [65, Theorem 4.2].

For a completely contractive Banach algebra $\mathcal{A}$, we let $\mathcal{A}^{e}:=\mathcal{A}^{o p} \widehat{\otimes} \mathcal{A}$ be its enveloping completely contractive Banach algebra, where $\mathcal{A}^{\text {op }}$ is the opposite algebra of $\mathcal{A}$, with multiplication given by $a \cdot_{\text {op }} b=b a, a, b \in \mathcal{A}$. There is a one-to-one correspondence between operator $\mathcal{A}$-bimodules $X$ and right operator $\mathcal{A}^{e}$-modules via

$$
x \cdot(a \otimes b)=a \cdot x \cdot b, \quad x \in X, a, b \in \mathcal{A} .
$$


Thus, two-sided homological properties of $\mathcal{A}$-bimodules, such as injectivity, may be studied via its one-sided counterpart over the enveloping algebra $\mathcal{A}^{e}$. As a result, we obtain bimodule versions of Propositions 4.3.1 and 4.3.5, which we state in the special case of $X=\mathcal{A}^{*} \in \mathcal{A}-\bmod -\mathcal{A}$.

Proposition 4.3.7. Let $\mathcal{A}$ be a completely contractive Banach algebra. If $\mathcal{A}$ is $C_{1}$ projective in $\mathbb{C}-\bmod -\mathbb{C}$ and is relatively $C_{2}$-projective in $\mathcal{A}-\bmod -\mathcal{A}$, then $\mathcal{A}$ is $C_{1} C_{2}$-projective in $\mathcal{A}-\bmod -\mathcal{A}$.

Proposition 4.3.8. Let $\mathcal{A}$ be a completely contractive Banach algebra. If $\mathcal{A}^{*}$ is $C_{1}$ injective in $\mathbb{C}-\bmod -\mathbb{C}$ and relatively $C_{2}$-injective in $\mathcal{A}-\bmod -\mathcal{A}$, then $\mathcal{A}^{*}$ is $C_{1} C_{2}$-injective in $\mathcal{A}-\bmod -\mathcal{A}$.

We now consider the injectivity of $\mathcal{B}\left(L^{2}(\mathbb{G})\right)$ as a $\mathcal{T}\left(L^{2}(\mathbb{G})\right)$-module. In what follows we let $\mathcal{T}_{\triangleright}$ denote the Banach algebra $\left(\mathcal{T}\left(L^{2}(\mathbb{G})\right), \triangleright\right)$. Note that if $T \in \mathcal{B}\left(L^{2}(\mathbb{G})\right)$ satisfies $\rho \triangleright T=0$ for all $\rho \in \mathcal{T}\left(L^{2}(\mathbb{G})\right)$, then $0=\langle\rho \triangleright T, \omega\rangle=\langle T, \omega \triangleright \rho\rangle$ for all $\rho, \omega \in \mathcal{T}\left(L^{2}(\mathbb{G})\right)$, entailing $T=0$ by (4.1) Thus, $\mathcal{B}\left(L^{2}(\mathbb{G})\right)$ is faithful in $\mathcal{T}_{\triangleright}-\bmod$. By a similar argument it follows that $\mathcal{B}\left(L^{2}(\mathbb{G})\right)$ is also faithful in $\mathbf{m o d}-\mathcal{T}_{\triangleright}$.

Theorem 4.3.9. Let $\mathbb{G}$ be a locally compact quantum group. Then $\mathbb{G}$ is amenable if and only if $\mathcal{B}\left(L^{2}(\mathbb{G})\right)$ is 1-injective in $\mathcal{T}_{\triangleright}-\bmod$.

Proof. Suppose $\mathbb{G}$ is amenable, and let $m \in L^{\infty}(\mathbb{G})^{*}$ be a right invariant mean. Since $\mathcal{B}\left(L^{2}(\mathbb{G})\right)$ is a 1-injective operator space and is faithful in $\mathcal{T}_{\triangleright}-\bmod$, by Propositions 4.3.3 and 4.3.5 it suffices to provide a completely contractive morphism that is a left inverse for the map $\Delta: \mathcal{B}\left(L^{2}(\mathbb{G})\right) \rightarrow \mathcal{C B}\left(\mathcal{T}\left(L^{2}(\mathbb{G})\right), \mathcal{B}\left(L^{2}(\mathbb{G})\right)\right)$ given by

$$
\Delta(T)(\rho)=\rho \triangleright T, \quad T \in \mathcal{B}\left(L^{2}(\mathbb{G})\right), \rho \in \mathcal{T}\left(L^{2}(\mathbb{G})\right)
$$

Identifying $\mathcal{C B}\left(\mathcal{T}\left(L^{2}(\mathbb{G})\right), \mathcal{B}\left(L^{2}(\mathbb{G})\right)\right) \cong \mathcal{B}\left(L^{2}(\mathbb{G})\right) \bar{\otimes} \mathcal{B}\left(L^{2}(\mathbb{G})\right)$ via

$$
\langle\Phi, \rho \otimes \omega\rangle=\langle\Phi(\omega), \rho\rangle, \quad \Phi \in \mathcal{C} \mathcal{B}\left(\mathcal{T}\left(L^{2}(\mathbb{G})\right), \mathcal{B}\left(L^{2}(\mathbb{G})\right)\right), \rho, \omega \in \mathcal{T}\left(L^{2}(\mathbb{G})\right),
$$


one easily sees that $\Delta=\Gamma^{r}$, and that the corresponding left $\mathcal{T}_{\triangleright}$-module structure on $\mathcal{B}\left(L^{2}(\mathbb{G})\right) \bar{\otimes} \mathcal{B}\left(L^{2}(\mathbb{G})\right)$ is given by

$$
\rho \neg A=(\mathrm{id} \otimes \mathrm{id} \otimes \rho)\left(\mathrm{id} \otimes \Gamma^{r}\right)(A), \quad A \in \mathcal{B}\left(L^{2}(\mathbb{G})\right) \bar{\otimes} \mathcal{B}\left(L^{2}(\mathbb{G})\right), \rho \in \mathcal{T}\left(L^{2}(\mathbb{G})\right) .
$$

It then remains to find a completely contractive morphism $\Phi: \mathcal{B}\left(L^{2}(\mathbb{G})\right) \bar{\otimes} \mathcal{B}\left(L^{2}(\mathbb{G})\right) \rightarrow$ $\mathcal{B}\left(L^{2}(\mathbb{G})\right)$ satisfying $\Phi \circ \Gamma^{r}=\operatorname{id}_{\mathcal{B}\left(L^{2}(\mathbb{G})\right)}$.

Let $\rho_{0} \in \mathcal{T}\left(L^{2}(\mathbb{G})\right)$ be a state. Then $m_{0}:=\rho_{0} \circ \Theta^{r}(m)$ is a state on $\mathcal{B}\left(L^{2}(\mathbb{G})\right)$, and we define $\Phi: \mathcal{B}\left(L^{2}(\mathbb{G})\right) \bar{\otimes} \mathcal{B}\left(L^{2}(\mathbb{G})\right) \rightarrow \mathcal{B}\left(L^{2}(\mathbb{G})\right)$ by

$$
\Phi(A)=\left(\mathrm{id} \otimes m_{0}\right)\left(V^{*} A V\right), \quad A \in \mathcal{B}\left(L^{2}(\mathbb{G})\right) \bar{\otimes} \mathcal{B}\left(L^{2}(\mathbb{G})\right) .
$$

Clearly, $\Phi$ is a complete contraction, and for $T \in \mathcal{B}\left(L^{2}(\mathbb{G})\right)$, we have

$$
\Phi\left(\Gamma^{r}(T)\right)=\Phi\left(V(T \otimes 1) V^{*}\right)=\left(\mathrm{id} \otimes m_{0}\right)(T \otimes 1)=T,
$$

so $\Phi$ is a left inverse for $\Gamma^{r}$. Fix $A \in \mathcal{B}\left(L^{2}(\mathbb{G})\right) \bar{\otimes} \mathcal{B}\left(L^{2}(\mathbb{G})\right)$ and $\rho \in \mathcal{T}\left(L^{2}(\mathbb{G})\right)$. Then, using the standard leg notation, we obtain

$$
\begin{aligned}
\Phi(\rho) A) & =\Phi\left((\mathrm{id} \otimes \mathrm{id} \otimes \rho)\left(V_{23} A_{12} V_{23}^{*}\right)\right) \\
& =\left(\mathrm{id} \otimes m_{0}\right)(\mathrm{id} \otimes \mathrm{id} \otimes \rho)\left(V_{12}^{*} V_{23} A_{12} V_{23}^{*} V_{12}\right) \\
& =\left(\mathrm{id} \otimes m_{0}\right)(\mathrm{id} \otimes \mathrm{id} \otimes \rho)\left(V_{13} V_{23} V_{12}^{*} A_{12} V_{12} V_{23}^{*} V_{13}^{*}\right) \quad(\text { by }(3.4)) \\
& =(\mathrm{id} \otimes \rho)\left(V\left(\mathrm{id} \otimes m_{0} \otimes \mathrm{id}\right)\left(V_{23} V_{12}^{*} A_{12} V_{12} V_{23}^{*}\right) V^{*}\right) \quad(\text { by }(2.5)) .
\end{aligned}
$$

Now, for any $\tau, \omega \in \mathcal{T}\left(L^{2}(\mathbb{G})\right)$, recalling that $\pi: \mathcal{T}\left(L^{2}(\mathbb{G})\right) \rightarrow L^{1}(\mathbb{G})$ denotes the canonical quotient map, we have 
$\left\langle\left(\mathrm{id} \otimes m_{0} \otimes \mathrm{id}\right)\left(V_{23} V_{12}^{*} A_{12} V_{12} V_{23}^{*}\right), \tau \otimes \omega\right\rangle=\left\langle\left(m_{0} \otimes \mathrm{id}\right) V\left((\tau \otimes \mathrm{id})\left(V^{*} A V\right) \otimes 1\right) V^{*}, \omega\right\rangle$

$$
\begin{aligned}
& =\left\langle m_{0}, \Theta^{r}(\pi(\omega))\left((\tau \otimes \mathrm{id}) V^{*} A V\right)\right\rangle \\
& =\left\langle\rho_{0}, \Theta^{r}(m) \circ \Theta^{r}(\pi(\omega))\left((\tau \otimes \mathrm{id}) V^{*} A V\right)\right\rangle \\
& =\left\langle\rho_{0}, \Theta^{r}(m \square \pi(\omega))\left((\tau \otimes \mathrm{id}) V^{*} A V\right)\right\rangle \\
& =\left\langle\rho_{0},\langle\omega, 1\rangle \Theta^{r}(m)\left((\tau \otimes \mathrm{id}) V^{*} A V\right)\right\rangle \\
& =\left\langle m_{0} \otimes \omega,(\tau \otimes \mathrm{id})\left(V^{*} A V\right) \otimes 1\right\rangle \\
& =\left\langle\left(\mathrm{id} \otimes m_{0} \otimes \mathrm{id}\right)\left(V^{*} A V \otimes 1\right), \tau \otimes \omega\right\rangle \\
& =\langle\Phi(A) \otimes 1, \tau \otimes \omega\rangle .
\end{aligned}
$$

Since $\tau$ and $\omega$ in $\mathcal{T}\left(L^{2}(\mathbb{G})\right)$ were arbitrary, it follows that

$$
\begin{aligned}
\Phi(\rho \triangleright A) & =(\mathrm{id} \otimes \rho)\left(V\left(\mathrm{id} \otimes m_{0} \otimes \mathrm{id}\right)\left(V_{23} V_{12}^{*} A_{12} V_{12} V_{23}^{*}\right) V^{*}\right) \\
& =(\mathrm{id} \otimes \rho)\left(V(\Phi(A) \otimes 1) V^{*}\right) \\
& =\rho \triangleright \Phi(A) .
\end{aligned}
$$

Conversely, if $\mathcal{B}\left(L^{2}(\mathbb{G})\right)$ is 1-injective in $\mathcal{T}_{\triangleright}-\bmod$, then it is relatively 1-injective in $\mathcal{T}_{\triangleright}-\bmod$, and by Proposition 4.3.3 there exists a completely contractive morphism $\Phi: \mathcal{B}\left(L^{2}(\mathbb{G})\right) \bar{\otimes} \mathcal{B}\left(L^{2}(\mathbb{G})\right) \rightarrow \mathcal{B}\left(L^{2}(\mathbb{G})\right)$ that is a left inverse to $\Gamma^{r}$. Then $\Gamma^{r} \circ \Phi$ is a projection of norm one onto the image of $\Gamma^{r}$. For any $A \in \mathcal{B}\left(L^{2}(\mathbb{G})\right) \bar{\otimes} \mathcal{B}\left(L^{2}(\mathbb{G})\right)$ and $\rho, \omega \in \mathcal{T}\left(L^{2}(\mathbb{G})\right)$, using the module property of $\Phi$ together with (2.5) we obtain

$$
\begin{aligned}
\left\langle\Gamma^{r} \circ \Phi(A), \omega \otimes \rho\right\rangle & =\left\langle(\operatorname{id} \otimes \rho)\left(\Gamma^{r}(\Phi(A))\right), \omega\right\rangle=\langle\rho \triangleright \Phi(A), \omega\rangle=\langle\Phi(\rho \triangleright A), \omega\rangle \\
& =\left\langle\Phi\left((\operatorname{id} \otimes \operatorname{id} \otimes \rho)\left(\operatorname{id} \otimes \Gamma^{r}\right)(A)\right), \omega\right\rangle \\
& =\left\langle(\operatorname{id} \otimes \rho)\left((\operatorname{id} \otimes \Phi) \circ\left(\operatorname{id} \otimes \Gamma^{r}\right)(A)\right), \omega\right\rangle \\
& =\left\langle(\operatorname{id} \otimes \Phi) \circ\left(\operatorname{id} \otimes \Gamma^{r}\right)(A), \omega \otimes \rho\right\rangle .
\end{aligned}
$$


Thus, $\Gamma^{r} \circ \Phi=(\Phi \otimes$ id $) \circ\left(\right.$ id $\left.\otimes \Gamma^{r}\right)$. Define a map $E: \mathcal{B}\left(L^{2}(\mathbb{G})\right) \rightarrow \mathcal{B}\left(L^{2}(\mathbb{G})\right)$ by

$$
E(T)=\Phi(T \otimes 1), \quad T \in \mathcal{B}\left(L^{2}(\mathbb{G})\right) .
$$

Then $E$ is a complete contraction, and for $T \in \mathcal{B}\left(L^{2}(\mathbb{G})\right)$ we have

$$
\begin{aligned}
\Gamma^{r}(E(T)) & =\Gamma^{r}(\Phi(T \otimes 1))=(\Phi \otimes \mathrm{id}) \circ\left(\mathrm{id} \otimes \Gamma^{r}\right)(T \otimes 1) \\
& =(\Phi \otimes \mathrm{id})(T \otimes 1 \otimes 1)=\Phi(T \otimes 1) \otimes 1 \\
& =E(T) \otimes 1,
\end{aligned}
$$

which by the standard argument shows that $E(T) \in L^{\infty}(\widehat{\mathbb{G}})$. Moreover, $E(\hat{x})=$ $\Phi(\hat{x} \otimes 1)=\Phi\left(\Gamma^{r}(\hat{x})\right)=\hat{x}$ for all $\hat{x} \in L^{\infty}(\widehat{\mathbb{G}})$, making $E$ a projection of norm one onto $L^{\infty}(\widehat{\mathbb{G}})$.

Since $\Gamma^{r} \circ \Phi$ is a projection of norm one onto $\Gamma^{r}\left(\mathcal{B}\left(L^{2}(\mathbb{G})\right)\right)$, it follows from [105] that

$$
\left(\Gamma^{r} \circ \Phi\right)\left(\Gamma^{r}(S) A \Gamma^{r}(T)\right)=\Gamma^{r}(S)\left(\Gamma^{r} \circ \Phi(A)\right) \Gamma^{r}(T)=\Gamma^{r}(S \Phi(A) T),
$$

which, by the injectivity of $\Gamma^{r}$, implies $\Phi\left(\Gamma^{r}(S) A \Gamma^{r}(T)\right)=S \Phi(A) T$ for all $S, T \in$ $\mathcal{B}\left(L^{2}(\mathbb{G})\right)$ and $A \in \mathcal{B}\left(L^{2}(\mathbb{G})\right) \bar{\otimes} \mathcal{B}\left(L^{2}(\mathbb{G})\right)$. Taking $A=x^{\prime} \otimes 1 \in L^{\infty}(\mathbb{G})^{\prime} \bar{\otimes} L^{\infty}(\mathbb{G})^{\prime}$ and $x \in L^{\infty}(\mathbb{G})$, we therefore have $\Phi\left(x^{\prime} \otimes 1\right) x=x \Phi\left(x^{\prime} \otimes 1\right)$. Consequently, $E\left(x^{\prime}\right)=$ $\Phi\left(x^{\prime} \otimes 1\right) \in L^{\infty}(\mathbb{G})^{\prime}$ for every $x^{\prime} \in L^{\infty}(\mathbb{G})^{\prime}$. Since $L^{\infty}(\mathbb{G})$ is standard in $\mathcal{B}\left(L^{2}(\mathbb{G})\right)$, there is a conjugate linear involution $J$ on $L^{2}(\mathbb{G})$ satisfying $J L^{\infty}(\mathbb{G}) J=L^{\infty}(\mathbb{G})^{\prime}$. Moreover, $J L^{\infty}(\widehat{\mathbb{G}}) J \subseteq L^{\infty}(\widehat{\mathbb{G}})\left[64\right.$, Proposition 2.1], so that $E_{J}: \mathcal{B}\left(L^{2}(\mathbb{G})\right) \rightarrow L^{\infty}(\widehat{\mathbb{G}})$ given by

$$
E_{J}(T)=J E(J T J) J, \quad T \in \mathcal{B}\left(L^{2}(\mathbb{G})\right)
$$

also defines a conditional expectation onto $L^{\infty}(\widehat{\mathbb{G}})$. Clearly, $E_{J}\left(L^{\infty}(\mathbb{G})\right) \subseteq L^{\infty}(\mathbb{G}) \cap$ $L^{\infty}(\widehat{\mathbb{G}})=\mathbb{C} 1$, so let $m \in L^{\infty}(\mathbb{G})^{*}$ be given by $m(x)=E_{J}(x)$. Then $m$ is a state and 
by the proof of Proposition 4.2.10 $E_{J} \in \mathcal{C B}_{\mathcal{T}_{\triangleleft}}\left(\mathcal{B}\left(L^{2}(\mathbb{G})\right)\right)$, thus

$$
\langle m, x \star \pi(\rho)\rangle=\langle m, x \triangleleft \rho\rangle=E_{J}(x \triangleleft \rho)=E_{J}(x) \triangleleft \rho=\langle\pi(\rho), 1\rangle\langle m, x\rangle
$$

for all $x \in L^{\infty}(\mathbb{G})$ and $\rho \in \mathcal{T}\left(L^{2}(\mathbb{G})\right)$. Hence, $\mathbb{G}$ is amenable.

By considering the category of dual operator left $\mathcal{T}\left(L^{2}(\mathbb{G})\right)$-modules with weak* continuous completely bounded morphisms, denoted $\mathcal{T}_{\triangleright}-$ nmod, we obtain the following characterization of compactness.

Corollary 4.3.10. Let $\mathbb{G}$ be a locally compact quantum group. Then $\mathbb{G}$ is compact if and only if $\mathcal{B}\left(L^{2}(\mathbb{G})\right)$ is relatively 1-injective in $\mathcal{T}_{\triangleright}$ - nmod.

Proof. If $\mathbb{G}$ is compact, there is a two-sided invariant mean $m \in L^{1}(\mathbb{G})$, and one may define a normal morphism as in equation (4.14) to produce a completely contractive left module inverse to $\Delta$, as defined in (4.13). Conversely, one may repeat the second half of the proof of Theorem 4.3.9 to obtain a normal conditional expectation from $\mathcal{B}\left(L^{2}(\mathbb{G})\right)$ onto $L^{\infty}(\widehat{\mathbb{G}})$ mapping $L^{\infty}(\mathbb{G})$ into $\mathbb{C} 1$. Then [56, Theorem 4.2] implies that $\widehat{\mathbb{G}}$ is discrete, whence $\mathbb{G}$ is compact.

Remark 4.3.11. At this point it is not clear whether we can replace relative 1injectivity with 1-injectivity in the statement of Corollary 4.3.10.

From the asymmetry of the completely contractive Banach algebra $\mathcal{T}_{\triangleright}$, one may expect a corresponding asymmetry between the left and right module structure of $\mathcal{B}\left(L^{2}(\mathbb{G})\right)$. The following theorem justifies this intuition and reveals a duality between the two: amenability of $\mathbb{G}$ is captured by left injectivity of $\mathcal{B}\left(L^{2}(\mathbb{G})\right)$ while amenability of $\widehat{\mathbb{G}}$ is captured by right injectivity of $\mathcal{B}\left(L^{2}(\mathbb{G})\right.$ ). Moreover, the theorem provides one of the main homological manifestations of duality of the thesis - amenability of $\widehat{\mathbb{G}}$ and 1-injectivity of $L^{\infty}(\mathbb{G})$ as an operator $L^{1}(\mathbb{G})$-module. 
Theorem 4.3.12. Let $\mathbb{G}$ be a locally compact quantum group. The following conditions are equivalent:

1. $\widehat{\mathbb{G}}$ is amenable;

2. $\mathcal{B}\left(L^{2}(\mathbb{G})\right)$ is 1-injective in $\bmod -\mathcal{T}_{\triangleright}$;

3. $L^{\infty}(\mathbb{G})$ is 1-injective in $\bmod -L^{1}(\mathbb{G})$.

Proof. (1) $\Rightarrow(2)$ : By [64, Proposition 2.15], the unitary operator $U \otimes U:=\widehat{J} J \otimes \widehat{J} J$ on $L^{2}(\mathbb{G}) \otimes L^{2}(\mathbb{G})$ intertwines the right fundamental unitaries of $\widehat{\mathbb{G}}$ and $\widehat{\mathbb{G}}^{\prime}$, denoted $\widehat{V}$ and $\widehat{V}^{\prime}$, respectively. One then obtains a one-to-one correspondence between invariant means on $L^{\infty}(\widehat{\mathbb{G}})$ and $L^{\infty}\left(\widehat{\mathbb{G}}^{\prime}\right)$ via conjugation with $U$, making $\widehat{\mathbb{G}}$ amenable if and only if $\widehat{\mathbb{G}}^{\prime}$ is. Thus, assuming amenability of $\widehat{\mathbb{G}}$, we let $\widehat{m}^{\prime}$ be a two-sided invariant mean on $L^{\infty}\left(\widehat{\mathbb{G}}^{\prime}\right)$. Similarly to the previous theorem, it suffices to provide a completely contractive morphism which is a left inverse to the map $\Delta: \mathcal{B}\left(L^{2}(\mathbb{G})\right) \rightarrow \mathcal{C B}\left(\mathcal{T}\left(L^{2}(\mathbb{G})\right), \mathcal{B}\left(L^{2}(\mathbb{G})\right)\right)$ given by

$$
\Delta(T)(\rho)=T \triangleright \rho, \quad T \in \mathcal{B}\left(L^{2}(\mathbb{G})\right), \rho \in \mathcal{T}\left(L^{2}(\mathbb{G})\right)
$$

In this case, we identify $\mathcal{C B}\left(\mathcal{T}\left(L^{2}(\mathbb{G})\right), \mathcal{B}\left(L^{2}(\mathbb{G})\right)\right) \cong \mathcal{B}\left(L^{2}(\mathbb{G})\right) \bar{\otimes} \mathcal{B}\left(L^{2}(\mathbb{G})\right)$ via

$$
\langle\Phi, \rho \otimes \omega\rangle=\langle\Phi(\rho), \omega\rangle, \quad \Phi \in \mathcal{C} \mathcal{B}\left(\mathcal{T}\left(L^{2}(\mathbb{G})\right), \mathcal{B}\left(L^{2}(\mathbb{G})\right)\right), \rho, \omega \in \mathcal{T}\left(L^{2}(\mathbb{G})\right)
$$

This ensures $\Delta=\Gamma^{r}$, and that the corresponding $\mathcal{T}\left(L^{2}(\mathbb{G})\right.$ )-module structure on $\mathcal{B}\left(L^{2}(\mathbb{G})\right) \bar{\otimes} \mathcal{B}\left(L^{2}(\mathbb{G})\right)$ is given by $A \bullet \rho=(\rho \otimes \mathrm{id} \otimes \mathrm{id})\left(\Gamma^{r} \otimes \mathrm{id}\right)(A)$ for $A \in \mathcal{B}\left(L^{2}(\mathbb{G})\right) \bar{\otimes} \mathcal{B}\left(L^{2}(\mathbb{G})\right)$ and $\rho \in \mathcal{T}\left(L^{2}(\mathbb{G})\right)$.

Take a normal state $\rho_{0} \in \mathcal{T}\left(L^{2}(\mathbb{G})\right)$, and define $\Phi: \mathcal{B}\left(L^{2}(\mathbb{G})\right) \bar{\otimes} \mathcal{B}\left(L^{2}(\mathbb{G})\right) \rightarrow$ $\mathcal{B}\left(L^{2}(\mathbb{G})\right)$ by

$$
\Phi(A)=\left(\mathrm{id} \otimes m_{0}\right)\left(V^{*} A V\right) \quad A \in \mathcal{B}\left(L^{2}(\mathbb{G})\right) \bar{\otimes} \mathcal{B}\left(L^{2}(\mathbb{G})\right),
$$


where $m_{0}:=\rho_{0} \circ \widehat{\Theta}^{\prime r}\left(\widehat{m}^{\prime}\right)$ is a state on $\mathcal{B}\left(L^{2}(\mathbb{G})\right)$, and $\widehat{\Theta}^{\prime r}$ denotes the representation (4.10) of LUC $\left(\widehat{\mathbb{G}}^{\prime}\right)^{*}$. Clearly, $\Phi$ is a completely contractive left inverse to $\Gamma^{r}$. To show that $\Phi$ is also a morphism we follow along similar lines as in Theorem 4.3.9. Fix $A \in \mathcal{B}\left(L^{2}(\mathbb{G})\right) \bar{\otimes} \mathcal{B}\left(L^{2}(\mathbb{G})\right)$ and $\rho \in \mathcal{T}\left(L^{2}(\mathbb{G})\right)$. Then

$$
\begin{aligned}
\Phi(A \triangleright \rho) & =\Phi\left((\rho \otimes \mathrm{id} \otimes \mathrm{id})\left(V_{12} A_{13} V_{12}^{*}\right)\right) \\
& =\left(\mathrm{id} \otimes m_{0}\right)(\rho \otimes \mathrm{id} \otimes \mathrm{id})\left(V_{23}^{*} V_{12} A_{13} V_{12}^{*} V_{23}\right) \\
& =\left(\mathrm{id} \otimes m_{0}\right)(\rho \otimes \mathrm{id} \otimes \mathrm{id})\left(V_{12} V_{23}^{*} V_{13}^{*} A_{13} V_{13} V_{23} V_{12}^{*}\right) \\
& =(\rho \otimes \mathrm{id})\left(V\left(\mathrm{id} \otimes \mathrm{id} \otimes m_{0}\right)\left(V_{23}^{*} V_{13}^{*} A_{13} V_{13} V_{23}\right) V^{*}\right) .
\end{aligned}
$$

Denoting by $\widehat{\pi}^{\prime}$ the canonical quotient map $\mathcal{T}\left(L^{2}(\mathbb{G})\right) \cong \mathcal{T}\left(L^{2}\left(\widehat{\mathbb{G}}^{\prime}\right)\right) \rightarrow L^{1}\left(\widehat{\mathbb{G}}^{\prime}\right)$, and using the fact that $\widehat{V}^{\prime}=\sigma V^{*} \sigma$, where $\sigma$ is the flip map on $L^{2}(\mathbb{G}) \otimes_{2} L^{2}(\mathbb{G})$, for any $\tau, \omega \in \mathcal{T}\left(L^{2}(\mathbb{G})\right)$, we have

$$
\begin{aligned}
& \left\langle\left(\mathrm{id} \otimes \mathrm{id} \otimes m_{0}\right)\left(V_{23}^{*} V_{13}^{*} A_{13} V_{13} V_{23}\right), \tau \otimes \omega\right\rangle \\
& =\left\langle\left(\mathrm{id} \otimes \mathrm{id} \otimes m_{0}\right)\left(V_{23}^{*}(\sigma \otimes 1) V_{23}^{*} A_{23} V_{23}(\sigma \otimes 1) V_{23}\right), \tau \otimes \omega\right\rangle \\
& =\left\langle\left(\mathrm{id} \otimes \mathrm{id} \otimes m_{0}\right)\left(V_{13}^{*} V_{23}^{*} A_{23} V_{23} V_{13}\right), \omega \otimes \tau\right\rangle \\
& =\left\langle\left(\mathrm{id} \otimes m_{0}\right)\left(V^{*}\left(1 \otimes(\tau \otimes \mathrm{id})\left(V^{*} A V\right)\right) V\right), \omega\right\rangle \\
& =\left\langle\left(m_{0} \otimes \mathrm{id}\right)\left(\widehat{V}^{\prime}\left((\tau \otimes \mathrm{id})\left(V^{*} A V\right) \otimes 1\right) \widehat{V}^{\prime *}\right), \omega\right\rangle \\
& =\left\langle m_{0}, \widehat{\Theta}^{\prime r}\left(\widehat{\pi}^{\prime}(\omega)\right)\left((\tau \otimes \mathrm{id})\left(V^{*} A V\right)\right)\right\rangle \\
& =\left\langle\rho_{0}, \widehat{\Theta}^{\prime r}\left(\widehat{m}^{\prime}\right) \circ \widehat{\Theta}^{\prime r}\left(\widehat{\pi}^{\prime}(\omega)\right)\left((\tau \otimes \mathrm{id})\left(V^{*} A V\right)\right)\right\rangle \\
& =\left\langle\rho_{0}, \widehat{\Theta}^{\prime r}\left(\widehat{m}^{\prime} \widehat{\square}^{\prime} \widehat{\pi}^{\prime}(\omega)\right)\left((\tau \otimes \mathrm{id})\left(V^{*} A V\right)\right)\right\rangle \\
& =\left\langle\rho_{0},\langle\omega, 1\rangle \Theta^{r}\left(\widehat{m}^{\prime}\right)\left((\tau \otimes \mathrm{id})\left(V^{*} A V\right)\right)\right\rangle \\
& =\left\langle m_{0} \otimes \omega,(\tau \otimes \mathrm{id})\left(V^{*} A V\right) \otimes 1\right\rangle \\
& =\left\langle\left(\mathrm{id} \otimes m_{0} \otimes \mathrm{id}\right)\left(V^{*} A V \otimes 1\right), \tau \otimes \omega\right\rangle \\
& =\langle\Phi(A) \otimes 1, \tau \otimes \omega\rangle .
\end{aligned}
$$


As $\tau$ and $\omega$ were arbitrary, we have

$$
\begin{aligned}
\Phi(A \triangleright \rho) & =(\rho \otimes \mathrm{id})\left(V\left(\mathrm{id} \otimes \mathrm{id} \otimes m_{0}\right)\left(V_{23}^{*} V_{13}^{*} A_{13} V_{13} V_{23}\right) V^{*}\right) \\
& =(\rho \otimes \mathrm{id})\left(V(\Phi(A) \otimes 1) V^{*}\right) \\
& =\Phi(A) \triangleright \rho .
\end{aligned}
$$

$(2) \Rightarrow(3)$ : If $\mathcal{B}\left(L^{2}(\mathbb{G})\right)$ is 1 -injective in $\bmod -\mathcal{T}_{\triangleright}$, there exists a completely contractive morphism $\Phi: \mathcal{B}\left(L^{2}(\mathbb{G})\right) \bar{\otimes} \mathcal{B}\left(L^{2}(\mathbb{G})\right) \rightarrow \mathcal{B}\left(L^{2}(\mathbb{G})\right)$ such that $\Phi \circ \Gamma^{r}=$ $\operatorname{id}_{\mathcal{B}\left(L^{2}(\mathbb{G})\right)}$. By the proof of Proposition 4.1.6, it follows that $\Phi(\rho \widehat{\boldsymbol{\nabla}} X)=\rho \widehat{\triangleright} \Phi(X)$, where the left module action is given by

$$
\rho \widehat{\boldsymbol{\Sigma}} X=(\mathrm{id} \otimes \rho \otimes \mathrm{id})\left(\widehat{\Gamma}^{r} \otimes \mathrm{id}\right)(X), \quad \rho \in \mathcal{T}\left(L^{2}(\mathbb{G})\right), \quad X \in \mathcal{B}\left(L^{2}(\mathbb{G})\right) \bar{\otimes} \mathcal{B}\left(L^{2}(\mathbb{G})\right) .
$$

Furthermore, the proof of Corollary 4.1.7 entails $\Phi\left(L^{\infty}(\mathbb{G}) \bar{\otimes} \mathcal{B}\left(L^{2}(\mathbb{G})\right)\right) \subseteq L^{\infty}(\mathbb{G})$. Since $\Gamma^{l}: \mathcal{B}\left(L^{2}(\mathbb{G})\right) \rightarrow L^{\infty}(\mathbb{G}) \bar{\otimes} \mathcal{B}\left(L^{2}(\mathbb{G})\right)$, the composition $\Phi \circ \Gamma^{l}$ therefore maps into $L^{\infty}(\mathbb{G})$. Moreover, if $x \in L^{\infty}(\mathbb{G})$ then $\Phi \circ \Gamma^{l}(x)=\Phi \circ \Gamma^{r}(x)=x$, making $\Phi \circ \Gamma^{l}$ a projection of norm one from $\mathcal{B}\left(L^{2}(\mathbb{G})\right)$ onto $L^{\infty}(\mathbb{G})$. Thus, $L^{\infty}(\mathbb{G})$ is 1-injective in $\bmod -\mathbb{C}$.

Next, consider the map $\Psi=\left.\Phi\right|_{L^{\infty}(\mathbb{G}) \bar{\otimes} L^{\infty}(\mathbb{G})}: L^{\infty}(\mathbb{G}) \bar{\otimes} L^{\infty}(\mathbb{G}) \rightarrow L^{\infty}(\mathbb{G})$. Since the right module action of $\left(\mathcal{T}\left(L^{2}(\mathbb{G})\right), \triangleright\right)$ on $\mathcal{B}\left(L^{2}(\mathbb{G})\right)$ restricts to the canonical right $L^{1}(\mathbb{G})$-module action on $L^{\infty}(\mathbb{G})$, it follows that $\Psi$ is a completely contractive right $L^{1}(\mathbb{G})$-module map such that $\Psi \circ \Gamma=\operatorname{id}_{L^{\infty}(\mathbb{G})}$. Since $L^{\infty}(\mathbb{G})$ is faithful in $\bmod -L^{1}(\mathbb{G})$, Proposition 4.3.3 entails the relative 1-injectivity of $L^{\infty}(\mathbb{G})$ in $\bmod -L^{1}(\mathbb{G})$, and therefore the 1-injectivity of $L^{\infty}(\mathbb{G})$ in $\bmod -L^{1}(\mathbb{G})$ by Proposition 4.3.5.

$$
\begin{gathered}
(3) \Rightarrow(1) \text { : Viewing } \mathcal{B}\left(L^{2}(\mathbb{G})\right) \text { as a right operator } L^{1}(\mathbb{G}) \text {-module via } \\
T \triangleleft f=(f \otimes \mathrm{id}) \Gamma^{l}(T)=(f \otimes \mathrm{id}) W^{*}(1 \otimes T) W, \quad f \in L^{1}(\mathbb{G}), T \in \mathcal{B}\left(L^{2}(\mathbb{G})\right),
\end{gathered}
$$

as $L^{\infty}(\mathbb{G})$ is 1-injective in $\bmod -L^{1}(\mathbb{G})$, the identity on $L^{\infty}(\mathbb{G})$ extends to a com- 
pletely contractive morphism $E: \mathcal{B}\left(L^{2}(\mathbb{G})\right) \rightarrow L^{\infty}(\mathbb{G})$. Proposition 4.2.18 then entails the amenability of $\widehat{\mathbb{G}}$.

Remark 4.3.13. The proof that amenability of $\widehat{\mathbb{G}}$ implies 1-injectivity of $L^{\infty}(\mathbb{G})$ in $\bmod -L^{1}(\mathbb{G})$ in Theorem 4.3.12 relies on the $\mathcal{T}_{\triangleright}$-module structure of $\mathcal{B}\left(L^{2}(\mathbb{G})\right)$ and the commutation relation $(4.4)$ at the level of $\mathcal{T}\left(L^{2}(\mathbb{G})\right)$. At present it is not clear how to prove this implication without passing through $\mathcal{T}\left(L^{2}(\mathbb{G})\right)$.

Analogously, there is a left module version of Theorem 4.3.12 involving the left product $\triangleleft$.

Theorem 4.3.14. Let $\mathbb{G}$ be a locally compact quantum group. The following conditions are equivalent:

1. $\widehat{\mathbb{G}}$ is amenable;

2. $\mathcal{B}\left(L^{2}(\mathbb{G})\right)$ is 1-injective in $\mathcal{T}_{\triangleleft}-\bmod$;

3. $L^{\infty}(\mathbb{G})$ is 1-injective in $L^{1}(\mathbb{G})-\bmod$.

Several applications of Theorem 4.3.12 will be pursued in the next section. For now, let us highlight the special cases of commutative and co-commutative quantum groups, respectively.

Corollary 4.3.15. Let $G$ be a locally compact group. The following conditions hold:

1. $\mathcal{B}\left(L^{2}(G)\right)$ is 1-injective in $\bmod -\mathcal{T}_{\triangleright_{a}}$;

2. $\mathcal{B}\left(L^{2}(G)\right)$ is 1-injective in $\mathcal{T}_{\triangleleft a}-\bmod$;

3. $L^{\infty}(G)$ is 1-injective in $\bmod -L^{1}(G)$;

4. $L^{\infty}(G)$ is 1-injective in $L^{1}(G)-\bmod$.

We remark that conditions (3) and (4) in Corollary 4.3.15 are not entirely new, and can be derived by known methods (see [18, Theorem 2.4]). The next corollary, however, is entirely new. 
Corollary 4.3.16. Let $G$ be a locally compact group. The following conditions are equivalent:

1. $G$ is amenable;

2. $\mathcal{B}\left(L^{2}(G)\right)$ is 1-injective in $\bmod -\mathcal{T}_{\triangleright_{s}}$;

3. $\mathcal{B}\left(L^{2}(G)\right)$ is 1-injective in $\mathcal{T}_{\triangleleft_{s}}-\bmod$;

4. $\mathcal{L}(G)$ is 1-injective in $\bmod -A(G)$;

5. $\mathcal{L}(G)$ is 1-injective in $A(G)-\bmod$.

Remark 4.3.17. One cannot replace 1-injectivity with relative 1-injectivity in conditions (4) and (5) of Corollary 4.3.16. The relative 1-injectivity of $\mathcal{L}(G)$ as an operator $A(G)$-module is in fact equivalent to inner amenability of $G$, as we will show in Theorem 5.1.4.

We finish this section by connecting amenability of a locally compact quantum group $\mathbb{G}$ with injectivity of $L^{\infty}(\widehat{\mathbb{G}})$ as an operator $\mathcal{T}_{\triangleright}$-module. As it turns out, and will become apparent in the proof, in the case of $L^{\infty}(\widehat{\mathbb{G}})$ it suffices to consider relative injectivity.

Theorem 4.3.18. Let $\mathbb{G}$ be a locally compact quantum group. The following statements are equivalent:

1. $\mathbb{G}$ is amenable;

2. $L^{\infty}(\widehat{\mathbb{G}})$ is 1-injective in $\bmod -\mathcal{T}_{\triangleright}$;

3. $L^{\infty}(\widehat{\mathbb{G}})$ is relatively 1 -injective in $\bmod -\mathcal{T}_{\triangleright}$;

4. $L^{\infty}(\widehat{\mathbb{G}})$ is 1-injective in $\mathcal{T}_{\triangleright}-\bmod$;

5. $L^{\infty}(\widehat{\mathbb{G}})$ is relatively 1-injective in $\mathcal{T}_{\triangleright}-$ mod. 
Proof. (1) $\Rightarrow(2)$ : Observe that if $\hat{x} \in L^{\infty}(\widehat{\mathbb{G}})$ such that $0=\hat{x} \triangleright \rho=\langle\hat{x}, \rho\rangle 1$ for all $\rho \in \mathcal{T}\left(L^{2}(\mathbb{G})\right)$, then $\langle\hat{x}, \hat{f}\rangle=0$ for all $\hat{f} \in L^{1}(\widehat{\mathbb{G}})$, forcing $\hat{x}=0$. Thus, $L^{\infty}(\widehat{\mathbb{G}})$ is faithful in $\bmod -\mathcal{T}_{\triangleright}$. Since $\mathbb{G}$ is amenable, we know that $L^{\infty}(\widehat{\mathbb{G}})$ is a 1 -injective operator space by Theorem 4.2.2. By Propositions 4.3.3 and 4.3.5 it therefore suffices to provide a completely contractive morphism which is a left inverse to the map $\Delta^{r}: L^{\infty}(\widehat{\mathbb{G}}) \rightarrow \mathcal{C B}\left(\mathcal{T}\left(L^{2}(\mathbb{G})\right), L^{\infty}(\widehat{\mathbb{G}})\right)$ given by

$$
\Delta^{r}(\hat{x})(\rho)=\hat{x} \triangleright \rho, \quad \hat{x} \in L^{\infty}(\widehat{\mathbb{G}}), \rho \in \mathcal{T}\left(L^{2}(\mathbb{G})\right)
$$

Identifying $\mathcal{C B}\left(\mathcal{T}\left(L^{2}(\mathbb{G})\right), L^{\infty}(\widehat{\mathbb{G}})\right) \cong \mathcal{B}\left(L^{2}(\mathbb{G})\right) \bar{\otimes} L^{\infty}(\widehat{\mathbb{G}})$ via

$$
\langle\Psi, \rho \otimes \hat{f}\rangle=\langle\Psi(\rho), \hat{f}\rangle
$$

for $\Psi \in \mathcal{C B}\left(\mathcal{T}\left(L^{2}(\mathbb{G})\right), L^{\infty}(\widehat{\mathbb{G}})\right), \rho \in \mathcal{T}\left(L^{2}(\mathbb{G})\right)$ and $\hat{f} \in L^{1}(\widehat{\mathbb{G}})$, one easily sees that $\Delta^{r}(\hat{x})=\hat{x} \otimes 1$ for all $\hat{x} \in L^{\infty}(\widehat{\mathbb{G}})$, and the corresponding $\mathcal{T}_{\triangleright}$-module structure on $\mathcal{B}\left(L^{2}(\mathbb{G})\right) \bar{\otimes} L^{\infty}(\widehat{\mathbb{G}})$ is given by

$$
A \triangleright \rho=(\rho \otimes \mathrm{id} \otimes \mathrm{id})\left(\Gamma^{r} \otimes \mathrm{id}\right)(A), \quad A \in \mathcal{B}\left(L^{2}(\mathbb{G})\right) \bar{\otimes} L^{\infty}(\widehat{\mathbb{G}}), \rho \in \mathcal{T}\left(L^{2}(\mathbb{G})\right) .
$$

Since $\mathbb{G}$ is amenable, there exists a conditional expectation $E: \mathcal{B}\left(L^{2}(\mathbb{G})\right) \rightarrow L^{\infty}(\widehat{\mathbb{G}})$ that is a morphism in $\bmod -\mathcal{T}_{\triangleright}$ by Theorem 4.2.2. Fix a state $\hat{f} \in L^{1}(\widehat{\mathbb{G}})$, and define $\Phi^{r}: \mathcal{B}\left(L^{2}(\mathbb{G})\right) \bar{\otimes} L^{\infty}(\widehat{\mathbb{G}}) \rightarrow L^{\infty}(\widehat{\mathbb{G}})$ by

$$
\Phi^{r}(A)=E((\operatorname{id} \otimes \hat{f}) A), \quad A \in \mathcal{B}\left(L^{2}(\mathbb{G})\right) \bar{\otimes} L^{\infty}(\widehat{\mathbb{G}}) .
$$

Then $\Phi^{r}$ is a complete contraction, and for $\hat{x} \in L^{\infty}(\widehat{\mathbb{G}})$ we have

$$
\Phi^{r}\left(\Delta^{r}(\hat{x})\right)=\Phi^{r}(\hat{x} \otimes 1)=E(\hat{x})=\hat{x}
$$

so that $\Phi^{r}$ is a left inverse to $\Delta^{r}$. Moreover, for $A \in \mathcal{B}\left(L^{2}(\mathbb{G})\right) \bar{\otimes} L^{\infty}(\widehat{\mathbb{G}})$ and $\rho \in$ 
$\mathcal{T}\left(L^{2}(\mathbb{G})\right)$, we have

$$
\begin{aligned}
\Phi^{r}(A \triangleright \rho) & =\Phi^{r}\left((\rho \otimes \mathrm{id} \otimes \mathrm{id})\left(\Gamma^{r} \otimes \mathrm{id}\right)(A)\right)=E\left((\rho \otimes \mathrm{id}) \Gamma^{r}((\mathrm{id} \otimes \hat{f}) A)\right) \\
& =E(((\mathrm{id} \otimes \hat{f}) A) \triangleright \rho)=E((\mathrm{id} \otimes \hat{f}) A) \triangleright \rho=\Phi^{r}(A) \triangleright \rho .
\end{aligned}
$$

$(2) \Rightarrow(3)$ is trivial.

$(3) \Rightarrow(1)$ : Since $L^{\infty}(\widehat{\mathbb{G}})$ is relatively 1 -injective in $\bmod -\mathcal{T}_{\triangleright}$, there is a completely contractive morphism $\Phi^{r}: \mathcal{B}\left(L^{2}(\mathbb{G})\right) \bar{\otimes} L^{\infty}(\widehat{\mathbb{G}}) \rightarrow L^{\infty}(\widehat{\mathbb{G}})$ that is a left inverse to $\Delta^{r}$. Define $E: \mathcal{B}\left(L^{2}(\mathbb{G})\right) \rightarrow L^{\infty}(\widehat{\mathbb{G}})$ by $E(T)=\Phi^{r}(T \otimes 1)$ for all $T \in \mathcal{B}\left(L^{2}(\mathbb{G})\right)$. Then $E$ is a morphism, and for $\hat{x} \in L^{\infty}(\widehat{\mathbb{G}})$ we get

$$
E(\hat{x})=\Phi^{r}(\hat{x} \otimes 1)=\Phi^{r}\left(\Delta^{r}(\hat{x})\right)=\hat{x}
$$

so that $E$ is a projection of norm one onto $L^{\infty}(\widehat{\mathbb{G}})$. Theorem 4.2 .2 then entails the amenability of $\mathbb{G}$.

$(1) \Rightarrow(4)$ : As above, it follows that $L^{\infty}(\widehat{\mathbb{G}})$ is faithful in $\mathcal{T}_{\triangleright}-\bmod$, and that $L^{\infty}(\widehat{\mathbb{G}})$ is a 1-injective operator space. By Propositions 4.3.3 and 4.3.5 it therefore suffices to provide a completely contractive morphism which is a left inverse to $\Delta^{l}: L^{\infty}(\widehat{\mathbb{G}}) \rightarrow$ $\mathcal{C B}\left(\mathcal{T}\left(L^{2}(\mathbb{G})\right), L^{\infty}(\widehat{\mathbb{G}})\right)$ given by

$$
\Delta^{l}(\hat{x})(\rho)=\rho \triangleright \hat{x}, \quad \hat{x} \in L^{\infty}(\widehat{\mathbb{G}}), \rho \in \mathcal{T}\left(L^{2}(\mathbb{G})\right) .
$$

With the identification $\mathcal{C B}\left(\mathcal{T}\left(L^{2}(\mathbb{G})\right), L^{\infty}(\widehat{\mathbb{G}})\right) \cong \mathcal{B}\left(L^{2}(\mathbb{G})\right) \bar{\otimes} L^{\infty}(\widehat{\mathbb{G}})$, it follows that $\Delta^{l}(\hat{x})=1 \otimes \hat{x}$ for all $\hat{x} \in L^{\infty}(\widehat{\mathbb{G}})$ and that the corresponding $\mathcal{T}_{\triangleright}$-module structure on $\mathcal{B}\left(L^{2}(\mathbb{G})\right) \bar{\otimes} L^{\infty}(\widehat{\mathbb{G}})$ is given by $\rho \triangleright A=(\mathrm{id} \otimes \rho \otimes \mathrm{id})\left(\Gamma^{r} \otimes \mathrm{id}\right)(A)$ for $A \in$ $\mathcal{B}\left(L^{2}(\mathbb{G})\right) \bar{\otimes} L^{\infty}(\widehat{\mathbb{G}})$ and $\rho \in \mathcal{T}\left(L^{2}(\mathbb{G})\right)$. By amenability of $\mathbb{G}$, there exists a conditional expectation $E: \mathcal{B}\left(L^{2}(\mathbb{G})\right) \rightarrow L^{\infty}(\widehat{\mathbb{G}})$ that is a morphism in $\mathcal{T}_{\triangleright}-\bmod$ by Proposition 4.2.9. Fix a state $\hat{f} \in L^{1}(\widehat{\mathbb{G}})$, put $m:=\hat{f} \circ E \in \mathcal{B}\left(L^{2}(\mathbb{G})\right)^{*}$, and define 
$\Phi^{l}: \mathcal{B}\left(L^{2}(\mathbb{G})\right) \bar{\otimes} L^{\infty}(\widehat{\mathbb{G}}) \rightarrow L^{\infty}(\widehat{\mathbb{G}})$ by

$$
\Phi^{l}(A)=(m \otimes \mathrm{id})(A), \quad A \in \mathcal{B}\left(L^{2}(\mathbb{G})\right) \bar{\otimes} L^{\infty}(\widehat{\mathbb{G}})
$$

Clearly $\Phi^{l}$ is a completely contractive left inverse to $\Delta^{l}$. Furthermore, for $A \in$ $\mathcal{B}\left(L^{2}(\mathbb{G})\right) \bar{\otimes} L^{\infty}(\widehat{\mathbb{G}}), \rho \in \mathcal{T}\left(L^{2}(\mathbb{G})\right)$ and $\hat{g} \in L^{1}(\widehat{\mathbb{G}})$, we have

$$
\begin{aligned}
\left\langle\Phi^{l}(\rho \triangleright A), \hat{g}\right\rangle & =\langle(m \otimes \mathrm{id})(\rho \triangleright A), \hat{g}\rangle=\left\langle m,(\mathrm{id} \otimes \rho) \Gamma^{r}((\mathrm{id} \otimes \hat{g}) A)\right\rangle \\
& =\langle\hat{f}, E(\rho \triangleright((\mathrm{id} \otimes \hat{g}) A))\rangle=\langle\hat{f}, \rho \triangleright E((\mathrm{id} \otimes \hat{g}) A)\rangle \\
& =\langle\rho, 1\rangle\langle m,(\operatorname{id} \otimes \hat{g})(A)\rangle=\langle\rho, 1\rangle\left\langle\Phi^{l}(A), \hat{g}\right\rangle=\left\langle\rho \triangleright \Phi^{l}(A), \hat{g}\right\rangle .
\end{aligned}
$$

(4) $\Rightarrow(5)$ is trivial.

$(5) \Rightarrow(1)$ : Since $L^{\infty}(\widehat{\mathbb{G}})$ is relatively 1 -injective in $\mathcal{T}_{\triangleright}-\bmod$, there is a morphism $\Phi^{l}: \mathcal{B}\left(L^{2}(\mathbb{G})\right) \bar{\otimes} L^{\infty}(\widehat{\mathbb{G}}) \rightarrow L^{\infty}(\widehat{\mathbb{G}})$ that is a left inverse to $\Delta^{l}$. Define $E: \mathcal{B}\left(L^{2}(\mathbb{G})\right) \rightarrow$ $L^{\infty}(\widehat{\mathbb{G}})$ by $E(T)=\Phi^{l}(T \otimes 1)$ for all $T \in \mathcal{B}\left(L^{2}(\mathbb{G})\right)$. Since $E(1)=\Phi^{l}(1 \otimes 1)=$ $\Phi^{l}\left(\Delta^{l}(1)\right)=1, E$ is a unital morphism, and for any state $\hat{f} \in L^{1}(\widehat{\mathbb{G}})$, we have $1=\hat{f} \circ E(1) \leq\|\hat{f} \circ E\| \leq 1$, making $\hat{f} \circ E$ a state in $\mathcal{B}\left(L^{2}(\mathbb{G})\right)^{*}$. By the proof of Proposition 4.2.9, it then follows that the restriction of $\hat{f} \circ E$ to $L^{\infty}(\mathbb{G})$ is a left invariant mean.

Remark 4.3.19. Contrary to Remark 4.2.20, it is unclear whether we can replace 1-injectivity with $C$-injectivity for some $C>1$ in the statements of the results in this section.

\subsection{Applications}

It is a well-known fact in abstract harmonic analysis that closed subgroups of locally compact amenable groups are amenable (see [92, Theorem 1.2.7]). With Theorem 4.3.14 at our disposal, we may now generalize this fact to arbitrary locally compact 
quantum groups.

Let $\mathbb{G}$ and $\mathbb{H}$ be two locally compact quantum groups. Then $\mathbb{H}$ is said to be a closed quantum subgroup of $\mathbb{G}$ (in the sense of Vaes) if there exists a normal unital injective *-homomorphism $\gamma: L^{\infty}(\widehat{\mathbb{H}}) \rightarrow L^{\infty}(\widehat{\mathbb{G}})$ satisfying

$$
(\gamma \otimes \gamma) \circ \widehat{\Gamma}_{\mathbb{H}}=\widehat{\Gamma}_{\mathbb{G}} \circ \gamma
$$

This is not the original definition of Vaes (cf. [108, Definition 2.5]), but was shown to be equivalent in [23, Theorem 3.3]. With this definition, we have an analog of the Herz restriction theorem [46] for quantum groups, that is, $\gamma_{*}: L^{1}(\widehat{\mathbb{G}}) \rightarrow L^{1}(\widehat{\mathbb{H}})$ is a complete quotient map [23, Theorem 3.7]. Indeed, if $\mathbb{G}$ and $\mathbb{H}$ are commutative, with underlying locally compact groups $G$ and $H$, the map $\gamma$ is nothing but the canonical inclusion

$$
\mathcal{L}(H) \ni \lambda_{H}(s) \mapsto \lambda_{G}(s) \in \mathcal{L}(G),
$$

where $\lambda_{H}$ and $\lambda_{G}$ are the left regular representations of $H$ and $G$, respectively. Its pre-adjoint $\gamma_{*}$ is then the canonical quotient map $A(G) \rightarrow A(H)$ coming from the classical Herz restriction theorem.

Remark 4.4.1. There is an a priori weaker notion of closed quantum subgroup of a locally compact quantum group $\mathbb{G}$ due to Woronowicz [23, Definition 3.2]. In what follows, we restrict ourselves to Vaes' definition, so that a closed quantum subgroup of a locally compact quantum group will always refer to the definition (4.17) given above.

Theorem 4.4.2. Let $\mathbb{G}$ and $\mathbb{H}$ be two locally compact quantum groups such that $\mathbb{H}$ is a closed quantum subgroup of $\mathbb{G}$. If $\mathbb{G}$ is amenable then $\mathbb{H}$ is amenable.

Proof. Since $\mathbb{G}$ is amenable, Theorem 4.3 .14 entails the 1-injectivity of $L^{\infty}(\widehat{\mathbb{G}})$ in $L^{1}(\widehat{\mathbb{G}})-\bmod$. The space $\mathcal{B}\left(L^{2}(\mathbb{H})\right)=\mathcal{B}\left(L^{2}(\widehat{\mathbb{H}})\right)$ becomes a left operator $L^{1}(\widehat{\mathbb{G}})$ - 
module via:

$$
\hat{f} \triangleright_{\widehat{\mathbb{G}}} T=\gamma_{*}(\hat{f}) \triangleright_{\widehat{\mathbb{H}}} T=\left(\mathrm{id} \otimes \gamma_{*}(\hat{f})\right) \widehat{\Gamma}_{\widehat{\mathbb{H}}}^{r}(T), \quad \hat{f} \in L^{1}(\widehat{\mathbb{G}}), T \in \mathcal{B}\left(L^{2}(\mathbb{H})\right) .
$$

Clearly, $L^{\infty}(\widehat{\mathbb{H}})$ is an $L^{1}(\widehat{\mathbb{G}})$-submodule of $\mathcal{B}\left(L^{2}(\mathbb{H})\right)$ and $\gamma: L^{\infty}(\widehat{\mathbb{H}}) \rightarrow L^{\infty}(\widehat{\mathbb{G}})$ is a left $L^{1}(\widehat{\mathbb{G}})$-module map. Thus, we may extend $\gamma$ to a completely contractive left $L^{1}(\widehat{\mathbb{G}})$ module map $\widetilde{\gamma}: \mathcal{B}\left(L^{2}(\mathbb{H})\right) \rightarrow L^{\infty}(\widehat{\mathbb{G}})$. Then $\widetilde{\gamma}$ is a unital complete contraction and thus completely positive. Moreover, $L^{\infty}(\widehat{\mathbb{H}})$ is contained in the multiplicative domain of $\widetilde{\gamma}$ (as it extends a ${ }^{*}$-homomorphism), so the bimodule property of completely positive maps over their multiplicative domains ensures that

$$
\widetilde{\gamma}(\hat{x} T \hat{y})=\gamma(\hat{x}) \widetilde{\gamma}(T) \gamma(\hat{y}), \quad \hat{x}, \hat{y} \in L^{\infty}(\widehat{\mathbb{H}}), T \in \mathcal{B}\left(L^{2}(\mathbb{H})\right)
$$

Since $\widetilde{\gamma}$ is a left $L^{1}(\widehat{\mathbb{G}})$-module map, for $x \in L^{\infty}(\mathbb{H})$ and $\hat{f}, \hat{g} \in L^{1}(\widehat{\mathbb{G}})$ we also have

$$
\begin{aligned}
\left\langle\widehat{\Gamma}_{\widehat{\mathbb{G}}}(\widetilde{\gamma}(x)), \hat{f} \otimes \hat{g}\right\rangle & =\langle\widetilde{\gamma}(x), \hat{f} \widehat{\star} \hat{g}\rangle \\
& =\left\langle\hat{g} \triangleright_{\widehat{\mathbb{G}}} \widetilde{\gamma}(x), \hat{f}\right\rangle \\
& =\left\langle\widetilde{\gamma}\left(\hat{g} \triangleright_{\widehat{\mathbb{G}}} x\right), \hat{f}\right\rangle \\
& =\left\langle\widetilde{\gamma}\left(\gamma_{*}(\hat{g}) \triangleright_{\widehat{\mathbb{H}}} x\right), \hat{f}\right\rangle \\
& =\langle\hat{g}, 1\rangle\langle\widetilde{\gamma}(x), \hat{f}\rangle \\
& =\langle\widetilde{\gamma}(x) \otimes 1, \hat{f} \otimes \hat{g}\rangle .
\end{aligned}
$$

The standard argument then shows that $\widetilde{\gamma}(x) \in L^{\infty}(\widehat{\mathbb{G}}) \cap L^{\infty}(\mathbb{G})=\mathbb{C} 1$.

Now, consider the space $X:=\widetilde{\gamma}^{-1}\left(\gamma\left(L^{\infty}(\widehat{\mathbb{H}})\right)\right) \subseteq \mathcal{B}\left(L^{2}(\mathbb{H})\right)$. By above, this is a closed operator $L^{\infty}(\widehat{\mathbb{H}})$-submodule of $\mathcal{B}\left(L^{2}(\mathbb{H})\right)$ containing $L^{\infty}(\mathbb{H})$ and $L^{\infty}(\widehat{\mathbb{H}})$, where the canonical $L^{\infty}(\widehat{\mathbb{H}})$-bimodule action is given by multiplication. By restriction, we obtain a completely contractive $L^{\infty}(\widehat{\mathbb{H}})$-bimodule map $\left.\gamma^{-1} \circ \widetilde{\gamma}\right|_{X}: X \rightarrow L^{\infty}(\widehat{\mathbb{H}}) \subseteq$ $\mathcal{B}\left(L^{2}(\mathbb{H})\right)$. By Wittstock's bimodule extension theorem [116], this map extends to a completely contractive $L^{\infty}(\widehat{\mathbb{H}})$-bimodule map $\Phi: \mathcal{B}\left(L^{2}(\mathbb{H})\right) \rightarrow \mathcal{B}\left(L^{2}(\mathbb{H})\right)$. As in 
the proof of Proposition 4.2.10, it follows that $\Phi \in \mathcal{C B}_{\mathcal{T}_{\triangleleft \mathbb{H}}}\left(\mathcal{B}\left(L^{2}(\mathbb{H})\right)\right)$. Hence, for $x \in L^{\infty}(\mathbb{H})$, and $\rho \in \mathcal{T}\left(L^{2}(\mathbb{H})\right)$

$$
\begin{aligned}
\left(\gamma^{-1} \circ \widetilde{\gamma}\right)\left(x \triangleleft_{\mathbb{H}} \rho\right) & =\Phi\left(x \triangleleft_{\mathbb{H}} \rho\right)=\Phi(x) \triangleleft_{\mathbb{H}} \rho=\left(\left(\gamma^{-1} \circ \widetilde{\gamma}\right)(x)\right) \triangleleft_{\mathbb{H}} \rho \\
& =\langle\rho, 1\rangle\left(\gamma^{-1} \circ \widetilde{\gamma}\right)(x)
\end{aligned}
$$

as $\left(\gamma^{-1} \circ \widetilde{\gamma}\right)\left(L^{\infty}(\mathbb{H})\right)=\mathbb{C} 1$. Thus, $\left.\gamma^{-1} \circ \widetilde{\gamma}\right|_{L^{\infty}(\mathbb{H})}$ is a left invariant mean, and $\mathbb{H}$ is amenable.

Remark 4.4.3. By [94, Theorem 8] together with [23, Theorem 6.1] it follows that a compact quantum subgroup of a co-amenable quantum group is co-amenable. The corresponding question for arbitrary quantum subgroups remains open.

For a locally compact quantum group $\mathbb{G}$, the set of unitary elements $u \in L^{\infty}(\mathbb{G})$ satisfying $\Gamma(u)=u \otimes u$ forms a locally compact group under the relative weak* topology, called the intrinsic group of $\mathbb{G}$, and is denoted $\operatorname{Gr}(\mathbb{G})$. The character group of $\mathbb{G}$ is defined as $\widetilde{\mathbb{G}}:=\operatorname{Gr}(\widehat{\mathbb{G}})$. It follows that $\widetilde{\mathbb{G}_{a}} \cong G$, its underlying locally compact group, and $\widetilde{\mathbb{G}_{s}} \cong \widehat{G}$, the group of continuous characters on $G$. For more details on the character group and properties of the assignment $\mathbb{G} \mapsto \widetilde{\mathbb{G}}$ we refer the reader to $[57,22]$. Since $\widetilde{\mathbb{G}}$ is always a closed quantum subgroup of $\mathbb{G}[22$, Theorem 5.5], we obtain the following generalization of [57, Theorem 5.14] beyond discrete quantum groups.

Corollary 4.4.4. Let $\mathbb{G}$ be a locally compact quantum group. If $\mathbb{G}$ is amenable then $\widetilde{\mathbb{G}}$ is amenable.

In [42] Haagerup provided an elegant characterization of injective von Neumann algebras via decomposability of completely bounded maps. More specifically, a von Neumann algebra $M$ is injective if and only if $\mathcal{C B}(M)=\operatorname{span} \mathcal{C P}(M)$. The next result provides a similar decomposition for $L^{1}(\mathbb{G})$-module maps on $L^{\infty}(\mathbb{G})$ when $L^{\infty}(\mathbb{G})$ is 1-injective in $\bmod -L^{1}(\mathbb{G})$. 
Proposition 4.4.5. Let $\mathbb{G}$ be a locally compact quantum group. If $L^{\infty}(\mathbb{G})$ is 1 injective in $\bmod -L^{1}(\mathbb{G})$ (equivalently, $\widehat{\mathbb{G}}$ is amenable) then

$$
\mathcal{C B}_{L^{1}(\mathbb{G})}\left(L^{\infty}(\mathbb{G})\right)=\operatorname{span} \mathcal{C P}{L^{1}(\mathbb{G})}\left(L^{\infty}(\mathbb{G})\right)
$$

Proof. Viewing $M_{n}\left(L^{\infty}(\mathbb{G})\right)$ as an operator $L^{1}(\mathbb{G})$-module under the amplified action:

$$
\left[x_{i j}\right] \star f=\left[x_{i j} \star f\right], \quad\left[x_{i j}\right] \in M_{n}\left(L^{\infty}(\mathbb{G})\right), f \in L^{1}(\mathbb{G}),
$$

we claim that $M_{n}\left(L^{\infty}(\mathbb{G})\right)$ is 1 -injective in $\bmod -L^{1}(\mathbb{G})$ for any $n \in \mathbb{N}$. Indeed, the canonical morphism

$$
\Delta_{n}: M_{n}\left(L^{\infty}(\mathbb{G})\right) \rightarrow \mathcal{C B}\left(L^{1}(\mathbb{G}), M_{n}\left(L^{\infty}(\mathbb{G})\right)\right)=M_{n}\left(\mathcal{C B}\left(L^{1}(\mathbb{G}), L^{\infty}(\mathbb{G})\right)\right)
$$

is nothing but the $n^{\text {th }}$ amplification of $\Delta: L^{\infty}(\mathbb{G}) \rightarrow \mathcal{C B}\left(L^{1}(\mathbb{G}), L^{\infty}(\mathbb{G})\right)$, so the $n^{\text {th }}$ amplification of a completely contractive module left inverse of $\Delta$ (which exists by 1-injectivity of $L^{\infty}(\mathbb{G})$ ) provides a completely contractive module left inverse to $\Delta_{n}$. Since $M_{n}\left(L^{\infty}(\mathbb{G})\right)$ is 1-injective in $\bmod -\mathbb{C}[102$, Proposition XV.3.2], the claim follows from Propositions 4.3.3 and 4.3.5.

Now, let $\Phi \in \mathcal{C B}_{L^{1}(\mathbb{G})}\left(L^{\infty}(\mathbb{G})\right)$ be a complete contraction, and consider the Paulsen system $\mathcal{S} \subseteq M_{2}\left(L^{\infty}(\mathbb{G})\right)$ defined by

$$
\mathcal{S}=\left\{\left(\begin{array}{cc}
\alpha 1 & x \\
y & \beta 1
\end{array}\right) \mid x, y \in L^{\infty}(\mathbb{G}), \alpha, \beta \in \mathbb{C}\right\}
$$

Then $\mathcal{S}$ is an $L^{1}(\mathbb{G})$-submodule of $M_{2}\left(L^{\infty}(\mathbb{G})\right)$ and $\Phi$ gives rise to a unital completely positive $L^{1}(\mathbb{G})$-module map $\Phi_{\mathcal{S}}: \mathcal{S} \rightarrow M_{2}\left(L^{\infty}(\mathbb{G})\right)$ via off-diagonalization [79]:

$$
\Phi_{\mathcal{S}}\left(\left(\begin{array}{cc}
\alpha 1 & x \\
y & \beta 1
\end{array}\right)\right)=\left(\begin{array}{cc}
\alpha 1 & \Phi(x) \\
\Phi^{*}(y) & \beta 1
\end{array}\right), \quad\left(\begin{array}{cc}
\alpha 1 & x \\
y & \beta 1
\end{array}\right) \in \mathcal{S}
$$


where $\Phi^{*}(y)=\Phi\left(y^{*}\right)^{*}, y \in L^{\infty}(\mathbb{G})$. By 1-injectivity of $M_{2}\left(L^{\infty}(\mathbb{G})\right)$ in $\bmod -$ $L^{1}(\mathbb{G})$, the map $\Phi_{\mathcal{S}}$ extends to a completely contractive $L^{1}(\mathbb{G})$-module map $\widetilde{\Phi}$ : $M_{2}\left(L^{\infty}(\mathbb{G})\right) \rightarrow M_{2}\left(L^{\infty}(\mathbb{G})\right)$ such that

$$
\widetilde{\Phi}\left(\left(\begin{array}{ll}
1 & 0 \\
0 & 1
\end{array}\right)\right)=\left(\begin{array}{ll}
1 & 0 \\
0 & 1
\end{array}\right) .
$$

Hence, $\widetilde{\Phi}$ is completely positive and is of the form

$$
\widetilde{\Phi}\left(\left(\begin{array}{ll}
x_{11} & x_{12} \\
x_{21} & x_{22}
\end{array}\right)\right)=\left(\begin{array}{cc}
\Psi_{1}\left(x_{11}\right) & \Phi\left(x_{12}\right) \\
\Phi^{*}\left(x_{21}\right) & \Psi_{2}\left(x_{22}\right)
\end{array}\right), \quad\left[x_{i j}\right] \in M_{2}\left(L^{\infty}(\mathbb{G})\right)
$$

where $\Psi_{i} \in \mathcal{C} \mathcal{P}_{L^{1}(\mathbb{G})}\left(L^{\infty}(\mathbb{G})\right)$ is the associated map to $P_{i i} \circ \widetilde{\Phi} \circ P_{i i}$, and $P_{i i} \in$ $\mathcal{C} \mathcal{P}_{L^{1}(\mathbb{G})}\left(M_{2}\left(L^{\infty}(\mathbb{G})\right)\right)$ is the diagonal projection onto the $(i, i)^{t h}$ entry for $i=1,2$. By [29, Proposition 5.4.2], it follows that the map

$$
\left.\widetilde{\Phi}\right|_{L^{\infty}(\mathbb{G})}: L^{\infty}(\mathbb{G}) \ni x \mapsto\left(\begin{array}{cc}
\Psi_{1}(x) & \Phi(x) \\
\Phi^{*}(x) & \Psi_{2}(x)
\end{array}\right) \in M_{2}\left(L^{\infty}(\mathbb{G})\right)
$$

is a completely positive $L^{1}(\mathbb{G})$-module map. Thus, via polarization (as in [29, Proposition 5.4.1]), it follows that $\Phi \in \operatorname{span} \mathcal{C P}_{L^{1}(\mathbb{G})}\left(L^{\infty}(\mathbb{G})\right)$.

Remark 4.4.6. As the proof of Proposition 4.4.5 shows, when $L^{\infty}(\mathbb{G})$ is 1 -injective in $\bmod -L^{1}(\mathbb{G})$ we can decompose every element $\Phi \in \mathcal{C B}_{L^{1}(\mathbb{G})}\left(L^{\infty}(\mathbb{G})\right)$ into a linear combination of 4 completely positive $L^{1}(\mathbb{G})$-module maps.

Remark 4.4.7. It would be interesting to study the converse of Proposition 4.4.5, that is, does $\mathcal{C B}_{L^{1}(\mathbb{G})}\left(L^{\infty}(\mathbb{G})\right)=$ span $\mathcal{C P}_{L^{1}(\mathbb{G})}\left(L^{\infty}(\mathbb{G})\right)$ necessitate the amenability of $\widehat{\mathbb{G}}$ ? We believe this to be true, and are currently working towards a proof in collaboration with Zhong-Jin Ruan, who initially proposed the question.

For a locally compact group $G$, it is well-known that $B(G)=M_{c b} A(G)$ whenever $G$ is amenable [24, Corollary 1.8]. Using Proposition 4.4.5 together with [21, Theorem 
5.2] we can now generalize this implication to arbitrary locally compact quantum groups. We note that the same result was obtained under the a priori stronger assumption that $\mathbb{G}$ is co-amenable [49, Theorem 4.2].

Corollary 4.4.8. Let $\mathbb{G}$ be a locally compact quantum group. If $\widehat{\mathbb{G}}$ is amenable then

$$
C_{u}(\mathbb{G})^{*} \cong M_{c b}^{r}\left(L^{1}(\mathbb{G})\right)
$$

Proof. First, we claim that $\mathcal{C B}_{L^{1}(\mathbb{G})}\left(C_{0}(\mathbb{G}), L^{\infty}(\mathbb{G})\right)=\mathcal{C B}_{L^{1}(\mathbb{G})}\left(C_{0}(\mathbb{G})\right)$. One inclusion is obvious, so let $\Phi \in \mathcal{C B}_{L^{1}(\mathbb{G})}\left(C_{0}(\mathbb{G}), L^{\infty}(\mathbb{G})\right)$. Then the restriction of its adjoint $\left.\Phi^{*}\right|_{L^{1}(\mathbb{G})} \in{ }_{L^{1}(\mathbb{G})} \mathcal{C B}\left(L^{1}(\mathbb{G}), M(\mathbb{G})\right)={ }_{L^{1}(\mathbb{G})} \mathcal{C B}\left(L^{1}(\mathbb{G})\right)$, noting that $L^{1}(\mathbb{G})=$ $\left\langle L^{1}(\mathbb{G}) \star L^{1}(\mathbb{G})\right\rangle$ is a closed ideal in $M(\mathbb{G})$. Hence, $\left(\left.\Phi^{*}\right|_{L^{1}(\mathbb{G})}\right)^{*} \in \mathcal{C B}_{L^{1}(\mathbb{G})}^{\sigma}\left(L^{\infty}(\mathbb{G})\right)=$ $\mathcal{C B}_{L^{1}(\mathbb{G})}\left(C_{0}(\mathbb{G})\right)$ by [53, Proposition 4.1]. But

$$
\left\langle\left(\left.\Phi^{*}\right|_{L^{1}(\mathbb{G})}\right)^{*}(x), f\right\rangle=\left\langle x,\left.\Phi^{*}\right|_{L^{1}(\mathbb{G})}(f)\right\rangle=\left\langle x, \Phi^{*}(f)\right\rangle=\langle\Phi(x), f\rangle
$$

for all $x \in C_{0}(\mathbb{G})$ and $f \in L^{1}(\mathbb{G})$, so $\left(\left.\Phi^{*}\right|_{L^{1}(\mathbb{G})}\right)^{*}$ is an extension of $\Phi$ which leaves $C_{0}(\mathbb{G})$ invariant, hence so too does $\Phi$.

Letting $\mathcal{I}$ denote the complete isometry

$$
\mathcal{C B}_{L^{1}(\mathbb{G})}\left(C_{0}(\mathbb{G})\right) \ni \Phi \mapsto\left(\left.\Phi^{*}\right|_{L^{1}(\mathbb{G})}\right)^{*} \in \mathcal{C B}_{L^{1}(\mathbb{G})}^{\sigma}\left(L^{\infty}(\mathbb{G})\right)
$$

and $\mathcal{R}: \mathcal{C B}_{L^{1}(\mathbb{G})}\left(L^{\infty}(\mathbb{G})\right) \rightarrow \mathcal{C B}_{L^{1}(\mathbb{G})}\left(C_{0}(\mathbb{G}), L^{\infty}(\mathbb{G})\right)$ the completely contractive restriction map, it follows that $\mathcal{P}^{\sigma}:=\mathcal{I} \circ \mathcal{R}: \mathcal{C B}_{L^{1}(\mathbb{G})}\left(L^{\infty}(\mathbb{G})\right) \rightarrow \mathcal{C B}_{L^{1}(\mathbb{G})}^{\sigma}\left(L^{\infty}(\mathbb{G})\right)$ is a completely contractive projection onto $\mathcal{C B}_{L^{1}(\mathbb{G})}^{\sigma}\left(L^{\infty}(\mathbb{G})\right)$. Moreover, $\mathcal{P}^{\sigma}$ maps $\mathcal{C P}_{L^{1}(\mathbb{G})}\left(L^{\infty}(\mathbb{G})\right)$ onto $\mathcal{C} \mathcal{P}_{L^{1}(\mathbb{G})}^{\sigma}\left(L^{\infty}(\mathbb{G})\right)$.

Since $\widehat{\mathbb{G}}$ is amenable, $\mathcal{C B}_{L^{1}(\mathbb{G})}\left(L^{\infty}(\mathbb{G})\right)=\operatorname{span} \mathcal{C P}_{L^{1}(\mathbb{G})}\left(L^{\infty}(\mathbb{G})\right)$ by Proposition 4.4.5, so given $\Phi \in \mathcal{C B}_{L^{1}(\mathbb{G})}^{\sigma}\left(L^{\infty}(\mathbb{G})\right)$ there exist $\Phi_{i} \in \mathcal{C P}_{L^{1}(\mathbb{G})}\left(L^{\infty}(\mathbb{G})\right) i=1, \ldots, 4$ such 
that

$\frac{1}{4}\left(\Phi_{1}-\Phi_{2}+i\left(\Phi_{3}-\Phi_{4}\right)\right)=\Phi=\mathcal{P}^{\sigma}(\Phi)=\frac{1}{4}\left(\mathcal{P}^{\sigma}\left(\Phi_{1}\right)-\mathcal{P}^{\sigma}\left(\Phi_{2}\right)+i\left(\mathcal{P}^{\sigma}\left(\Phi_{3}\right)-\mathcal{P}^{\sigma}\left(\Phi_{4}\right)\right)\right)$

and it follows that $\mathcal{C B}_{L^{1}(\mathbb{G})}^{\sigma}\left(L^{\infty}(\mathbb{G})\right)=\operatorname{span} \mathcal{C P}_{L^{1}(\mathbb{G})}^{\sigma}\left(L^{\infty}(\mathbb{G})\right)$. By [21, Theorem $5.2]$, span $\mathcal{C P}_{L^{1}(\mathbb{G})}^{\sigma}\left(L^{\infty}(\mathbb{G})\right) \cong C_{u}(\mathbb{G})^{*}$, so we have $M_{c b}^{r}\left(L^{1}(\mathbb{G})\right) \cong \mathcal{C B}_{L^{1}(\mathbb{G})}^{\sigma}\left(L^{\infty}(\mathbb{G})\right) \cong$ $C_{u}(\mathbb{G})^{*}$.

The observations in the proof of Corollary 4.4.8 lead to the following new characterization of the predual of $M_{c b}^{r}\left(L^{1}(\mathbb{G})\right)$.

Proposition 4.4.9. Let $\mathbb{G}$ be a locally compact quantum group. Then

$$
Q_{c b}^{r}\left(L^{1}(\mathbb{G})\right) \cong C_{0}(\mathbb{G}) \widehat{\otimes}_{L^{1}(\mathbb{G})} L^{1}(\mathbb{G})
$$

completely isometrically.

Proof. As noted in the proof of Corollary 4.4.8, we have

$$
\mathcal{C B}_{L^{1}(\mathbb{G})}\left(C_{0}(\mathbb{G})\right)=\mathcal{C B}_{L^{1}(\mathbb{G})}\left(C_{0}(\mathbb{G}), L^{\infty}(\mathbb{G})\right)
$$

Thus, $\Theta^{r}: M_{c b}^{r}\left(L^{1}(\mathbb{G})\right) \cong \mathcal{C B}_{L^{1}(\mathbb{G})}\left(C_{0}(\mathbb{G}), L^{\infty}(\mathbb{G})\right)$ completely isometrically [53, Proposition 4.1]. We need to show that $\Theta^{r}$ is a weak ${ }^{*}$-weak* homeomorphism. Since

$$
\mathcal{C B}_{L^{1}(\mathbb{G})}\left(C_{0}(\mathbb{G}), L^{\infty}(\mathbb{G})\right) \cong\left(C_{0}(\mathbb{G}) \widehat{\otimes}_{L^{1}(\mathbb{G})} L^{1}(\mathbb{G})\right)^{*}
$$

weak* homeomorphically, and $\Theta^{r}$ is a completely isometric isomorphism, it suffices to show that $\Theta^{r}$ is weak* continuous on bounded sets (see [20, Lemma 10.1]). Let $\left(\hat{b}_{i}^{\prime}\right)_{i \in I}$ be a bounded net in $M_{c b}^{r}\left(L^{1}(\mathbb{G})\right)$ converging weak* to $\hat{b}^{\prime}$. By Proposition 3.7.2, for any $X \in C_{0}(\mathbb{G}) \otimes_{\min } K_{\infty}$ and $\rho \in L^{1}(\mathbb{G}) \widehat{\otimes} T_{\infty}$, we have $\Omega_{X, \rho} \in Q_{c b}^{r}\left(L^{1}(\mathbb{G})\right)$, where

$$
\left\langle\hat{a}^{\prime}, \Omega_{X, \rho}\right\rangle=\left\langle\left(\Theta^{r}\left(\hat{a}^{\prime}\right) \otimes \operatorname{id}_{K_{\infty}}\right)(X), \rho\right\rangle, \quad \hat{a}^{\prime} \in M_{c b}^{r}\left(L^{1}(\mathbb{G})\right) .
$$


In particular, take $y \in K_{\infty}$ and $\tau \in T_{\infty}$ such that $\langle y, \tau\rangle=1$, and let $X=x \otimes y$ and $\rho=f \otimes \tau$ for some $x \in C_{0}(\mathbb{G})$ and $f \in L^{1}(\mathbb{G})$. Then

$$
\begin{aligned}
\left\langle\Theta^{r}\left(\hat{b}_{i}^{\prime}\right)(x), f\right\rangle & =\left\langle\left(\Theta^{r}\left(\hat{b}_{i}^{\prime}\right) \otimes \operatorname{id}_{K_{\infty}}\right)(X), \rho\right\rangle \\
& =\left\langle\hat{b}_{i}^{\prime}, \Omega_{X, \rho}\right\rangle \\
& \rightarrow\left\langle\hat{b}^{\prime}, \Omega_{X, \rho}\right\rangle \\
& =\left\langle\left(\Theta^{r}\left(\hat{b}^{\prime}\right) \otimes \operatorname{id}_{K_{\infty}}\right)(X), \rho\right\rangle \\
& =\left\langle\Theta^{r}\left(\hat{b}^{\prime}\right)(x), f\right\rangle .
\end{aligned}
$$

Hence, $\left(\Theta^{r}\left(\hat{b}_{i}^{\prime}\right)\right)_{i \in I}$ converges point weak ${ }^{*}$ to $\Theta^{r}\left(\hat{b}^{\prime}\right)$ in $\mathcal{C B}\left(C_{0}(\mathbb{G}), L^{\infty}(\mathbb{G})\right)$. Letting $q: C_{0}(\mathbb{G}) \widehat{\otimes} L^{1}(\mathbb{G}) \rightarrow C_{0}(\mathbb{G}) \widehat{\otimes}_{L^{1}(\mathbb{G})} L^{1}(\mathbb{G})$ be the quotient map, and viewing $\Theta^{r}\left(\hat{b}_{i}^{\prime}\right) \in$ $\left(C_{0}(\mathbb{G}) \widehat{\otimes}_{L^{1}(\mathbb{G})} L^{1}(\mathbb{G})\right)^{*}$, for any $x_{1}, \ldots, x_{n} \in C_{0}(\mathbb{G})$ and $f_{1}, \ldots, f_{n} \in L^{1}(\mathbb{G})$ we have

$$
\begin{aligned}
\left\langle\Theta^{r}\left(\hat{b}_{i}^{\prime}\right), q\left(\sum_{k=1}^{n} x_{k} \otimes f_{k}\right)\right\rangle & =\sum_{k=1}^{n}\left\langle\Theta^{r}\left(\hat{b}_{i}^{\prime}\right)\left(x_{k}\right), f_{k}\right\rangle \\
& \rightarrow \sum_{k=1}^{n}\left\langle\Theta^{r}\left(\hat{b}^{\prime}\right)\left(x_{k}\right), f_{k}\right\rangle \\
& =\left\langle\Theta^{r}\left(\hat{b}^{\prime}\right), q\left(\sum_{k=1}^{n} x_{k} \otimes f_{k}\right)\right\rangle .
\end{aligned}
$$

By density of the image $q\left(C_{0}(\mathbb{G}) \otimes L^{1}(\mathbb{G})\right)$ of the algebraic tensor product $C_{0}(\mathbb{G}) \otimes$ $L^{1}(\mathbb{G})$ in $C_{0}(\mathbb{G}) \widehat{\otimes}_{L^{1}(\mathbb{G})} L^{1}(\mathbb{G})$, it follows that $\left(\Theta^{r}\left(\hat{b}_{i}^{\prime}\right)\right)_{i \in I}$ converges weak* to $\Theta^{r}\left(\hat{b}^{\prime}\right)$ in $\mathcal{C B}_{L^{1}(\mathbb{G})}\left(C_{0}(\mathbb{G}), L^{\infty}(\mathbb{G})\right)=\left(C_{0}(\mathbb{G}) \widehat{\otimes}_{L^{1}(\mathbb{G})} L^{1}(\mathbb{G})\right)^{*}$.

Remark 4.4.10. The identification of $Q_{c b}^{r}\left(L^{1}(\mathbb{G})\right)$ in Proposition 4.4 .9 appears to be new even in the co-commutative case, that is, for any locally compact group $G$ we have $Q_{c b}(G) \cong C_{\lambda}^{*}(G) \widehat{\otimes}_{A(G)} A(G)$.

For our next application, we give a simplified proof of the fact that amenability of a discrete quantum group implies co-amenability of its compact dual. For compact Kac algebras, this was shown by Ruan [90, Theorem 4.5] and was later generalized 
to arbitrary compact quantum groups by Tomatsu [104, Theorem 3.8]. Tomatsu's argument relies on the specific modular theory of discrete quantum groups in order to apply the Powers-Størmer inequality (see [40, Lemma 2.10] for instance) in a crucial step. Our argument below completely avoids modular theory and the Powers-Størmer inequality, and instead relies on a new homological approach.

Theorem 4.4.11. A compact quantum group $\mathbb{G}$ is co-amenable if and only if $\widehat{\mathbb{G}}$ is amenable.

Proof. Co-amenability of $\mathbb{G}$ always implies amenability of $\widehat{\mathbb{G}}[6$, Theorem 3.2], so assume $\widehat{\mathbb{G}}$ is amenable. By Theorem 4.3.12 we know that $L^{\infty}(\mathbb{G})$ is 1-injective in $\bmod -L^{1}(\mathbb{G})$. Let $\Phi: L^{\infty}(\mathbb{G}) \bar{\otimes} L^{\infty}(\mathbb{G}) \rightarrow L^{\infty}(\mathbb{G})$ be a completely contractive left inverse to $\Gamma$ which is a right $L^{1}(\mathbb{G})$-module map. As a unital complete contraction, $\Phi$ is completely positive and $\left.\Phi\right|_{C(\mathbb{G}) \otimes_{\min } C(\mathbb{G})} \neq 0$ since $C(\mathbb{G})$ is unital. By $[6$, Theorem 3.3] we also know that $C(\mathbb{G})$ is nuclear, so let $\left(\Psi_{a}\right)_{a \in A}$ be a net of finite-rank, unital completely positive maps converging to $\mathrm{id}_{C(\mathbb{G})}$ in the point-norm topology. For $a \in A$, consider the unital completely positive map $\Phi_{a}: C(\mathbb{G}) \rightarrow C(\mathbb{G})$ given by

$$
\Phi_{a}=\left.\Phi \circ\left(\mathrm{id} \otimes \Psi_{a}\right) \circ \Gamma\right|_{C(\mathbb{G})} .
$$

The fact that $\Phi_{a}$ maps into $C(\mathbb{G})$ can be established using the density of $\Gamma(C(\mathbb{G}))(C(\mathbb{G}) \otimes 1)$ in $C(\mathbb{G}) \otimes_{\min } C(\mathbb{G})$ (see [63, Corollary 6.11]) together with the $\Gamma\left(L^{\infty}(\mathbb{G})\right.$ )-module property of $\Phi$ (see the proof of Theorem 4.3.9). However, the invariance $\Phi_{a}(C(\mathbb{G})) \subseteq C(\mathbb{G})$ will be a byproduct of the following argument.

Since $\Psi_{a}$ is finite rank, there exists $x_{1}^{a}, \ldots, x_{n_{a}}^{a} \in C(\mathbb{G})$ and $\mu_{1}^{a}, \ldots \mu_{n_{a}}^{a} \in M(\mathbb{G})$ such that

$$
\Psi_{a}(x)=\sum_{n=1}^{n_{a}}\left\langle\mu_{n}^{a}, x\right\rangle x_{n}^{a}, \quad x \in C(\mathbb{G}), a \in A .
$$

For each $a \in A$, and $1 \leq n \leq n_{a}$, let $\Phi_{(a, n)}: C(\mathbb{G}) \rightarrow C(\mathbb{G})$ be defined by

$$
\Phi_{(a, n)}(x)=\Phi\left(x \otimes x_{n}^{a}\right), \quad x \in C(\mathbb{G}) .
$$


Then $\Phi_{(a, n)}$ is completely bounded with $\left\|\Phi_{(a, n)}\right\|_{c b} \leq\left\|x_{n}^{a}\right\|_{C(\mathbb{G})}$, and is a right $L^{1}(\mathbb{G})$ module map. Hence, $\Phi_{(a, n)} \in \mathcal{C B}_{L^{1}(\mathbb{G})}(C(\mathbb{G}))=\Theta^{r}\left(M_{c b}^{r}\left(L^{1}(\mathbb{G})\right)\right)$. By Corollary 4.4.8, $M_{c b}^{r}\left(L^{1}(\mathbb{G})\right)=C_{u}(\mathbb{G})^{*}$, so there exist $\nu_{n}^{a} \in C_{u}(\mathbb{G})^{*}$ such that $\Phi_{(a, n)}=\Theta^{r}\left(\nu_{n}^{a}\right)$.

Let $\alpha \in \operatorname{Irr}(\mathbb{G}), 1 \leq i, j \leq n_{\alpha}$. Then

$$
\begin{aligned}
\Phi_{a}\left(u_{i j}^{\alpha}\right) & =\sum_{k=1}^{n_{\alpha}} \Phi\left(\left(\mathrm{id} \otimes \Psi_{a}\right)\left(u_{i k}^{\alpha} \otimes u_{k j}^{\alpha}\right)\right) \\
& =\sum_{k=1}^{n_{\alpha}} \sum_{n=1}^{n_{a}}\left\langle\mu_{n}^{a}, u_{k j}^{\alpha}\right\rangle \Phi\left(u_{i k}^{\alpha} \otimes x_{n}^{a}\right) \\
& =\sum_{k=1}^{n_{\alpha}} \sum_{n=1}^{n_{a}}\left\langle\mu_{n}^{a}, u_{k j}^{\alpha}\right\rangle \Phi_{(a, n)}\left(u_{i k}^{\alpha}\right) \\
& =\sum_{k=1}^{n_{\alpha}} \sum_{n=1}^{n_{a}}\left\langle\mu_{n}^{a}, u_{k j}^{\alpha}\right) \Theta^{r}\left(\nu_{n}^{a}\right)\left(u_{i k}^{\alpha}\right) \\
& =\sum_{k=1}^{n_{\alpha}} \sum_{n=1}^{n_{a}} \Theta^{r}\left(\nu_{n}^{a}\right)\left(\left(\mathrm{id} \otimes \mu_{n}^{a}\right)\left(u_{i k}^{\alpha} \otimes u_{k j}^{\alpha}\right)\right) \\
& =\sum_{n=1}^{n_{a}} \Theta^{r}\left(\nu_{n}^{a}\right)\left(\left(\mathrm{id} \otimes \mu_{n}^{a}\right) \Gamma\left(u_{i j}^{\alpha}\right)\right) \\
& =\sum_{n=1}^{n_{a}} \Theta^{r}\left(\nu_{n}^{a}\right)\left(\Theta^{r}\left(\mu_{n}^{a}\right)\left(u_{i j}^{\alpha}\right)\right) \\
& =\sum_{n=1}^{n_{a}} \Theta^{r}\left(\nu_{n}^{a} \star \mu_{n}^{a}\right)\left(u_{i j}^{\alpha}\right) \\
& =\Theta^{r}\left(\sum_{n=1}^{n_{a}} \nu_{n}^{a} \star \mu_{n}^{a}\right)\left(u_{i j}^{\alpha}\right) .
\end{aligned}
$$

Letting $\mu_{a}=\sum_{n=1}^{n_{a}} \nu_{n}^{a} \star \mu_{n}^{a}$, it follows by density of matrix coefficients (see §3.6) that $\Phi_{a}=\Theta^{r}\left(\mu_{a}\right)$. Moreover, we have $\mu_{a} \in M(\mathbb{G})$ as $M(\mathbb{G})$ is a two-sided ideal in $C_{u}(\mathbb{G})^{*}$. Since $\Theta^{r}$ is a (complete) isometry,

$$
\left\|\mu_{a}\right\|_{M(\mathbb{G})}=\left\|\Theta^{r}\left(\mu_{a}\right)\right\|_{c b}=\left\|\Phi_{a}\right\|_{c b}=1, \quad a \in A,
$$


and since $\Phi_{a}$ converges to $\mathrm{id}_{C(\mathbb{G})}$ in the point-norm topology it follows that

$$
\mu_{a} \star x=\Theta^{r}\left(\mu_{a}\right)(x) \rightarrow x, \quad x \in C(\mathbb{G}) .
$$

Let $\mu$ be a weak* cluster point of $\left(\mu_{a}\right)_{a \in A}$ in the unit ball of $M(\mathbb{G})=C(\mathbb{G})^{*}$. Then $\mu$ is a right identity of $M(\mathbb{G})$. The restricted unitary antipode $R$ maps $C(\mathbb{G})$ into $C(\mathbb{G})$ and satisfies $R^{*}(\mu \star \nu)=R^{*}(\nu) \star R^{*}(\mu)$ for all $\nu \in M(\mathbb{G})$. Hence, $R^{*}(\mu)$ is a left identity of $M(\mathbb{G})$. It follows that $\varepsilon:=\mu+R^{*}(\mu)-\mu \star R^{*}(\mu)$ is an identity for $M(\mathbb{G})$. Hence, $\mathbb{G}$ is co-amenable by [6, Theorem 3.1].

Given a completely contractive Banach algebra $\mathcal{A}$ with a contractive approximate identity, any essential module $X \in \bmod -\mathcal{A}$ is induced by [20, Proposition 6.4]. Since a locally compact quantum group $\mathbb{G}$ is co-amenable if and only if $L^{1}(\mathbb{G})$ has a contractive approximate identity [48, Theorem 2], the next proposition supports the idea that our methods may be applicable to the general duality problem of amenability and co-amenability.

Proposition 4.4.12. Let $\mathbb{G}$ be a locally compact quantum group for which the dual $\widehat{\mathbb{G}}$ is amenable. Then for any closed right ideal $I \unlhd L^{1}(\mathbb{G})$, the multiplication map yields a completely isometric isomorphism $\widetilde{m}_{I}: I \widehat{\otimes}_{L^{1}(\mathbb{G})} L^{1}(\mathbb{G}) \cong\left\langle I \star L^{1}(\mathbb{G})\right\rangle$. In particular, if $I$ is essential, then $I \widehat{\otimes}_{L^{1}(\mathbb{G})} L^{1}(\mathbb{G}) \cong I$, that is, $I$ is an induced right $L^{1}(\mathbb{G})$-module.

Proof. First, note that for any self-induced completely contractive Banach algebra $\mathcal{A}$ and any closed right ideal $J \unlhd \mathcal{A}$, we have $\widetilde{m}_{\mathcal{A} / J}:(\mathcal{A} / J) \widehat{\otimes}_{\mathcal{A}} \mathcal{A} \cong \mathcal{A} /\langle J \cdot \mathcal{A}\rangle$, completely isometrically. Indeed, identifying $(\mathcal{A} /\langle J \cdot \mathcal{A}\rangle)^{*}=\langle J \cdot \mathcal{A}\rangle^{\perp} \subseteq \mathcal{A}^{*}$, it follows that $\left(\widetilde{m}_{\mathcal{A} / J}\right)^{*}:\langle J \cdot \mathcal{A}\rangle^{\perp} \rightarrow\left((\mathcal{A} / J) \widehat{\otimes}_{\mathcal{A}} \mathcal{A}\right)^{*}=\left(N_{\mathcal{A} / J}\right)^{\perp}$ is equal to $\left.\left(\widetilde{m}_{\mathcal{A}}\right)^{*}\right|_{\langle J \cdot \mathcal{A}\rangle}$. In particular, $\left(\widetilde{m}_{\mathcal{A} / J}\right)^{*}$ is a complete isometry. Letting $q: \mathcal{A} \rightarrow \mathcal{A} / J$ be the complete quotient map, if $X \in\left(N_{\mathcal{A} / J}\right)^{\perp}$ then $\left(q^{*} \otimes\right.$ id $)(X) \in\left(N_{\mathcal{A}}\right)^{\perp}$, so there exists $F \in \mathcal{A}^{*}$ such that $\left(q^{*} \otimes \mathrm{id}\right)(X)=\left(\widetilde{m}_{\mathcal{A}}\right)^{*}(F)$ as $\mathcal{A}$ is self-induced. Clearly, $F \in\langle J \cdot \mathcal{A}\rangle^{\perp}$, so $\left(\widetilde{m}_{\mathcal{A} / J}\right)^{*}$ is also surjective. 
By above, it follows that $\widetilde{m}_{L^{1}(\mathbb{G}) / I}:\left(L^{1}(\mathbb{G}) / I\right) \widehat{\otimes}_{L^{1}(\mathbb{G})} L^{1}(\mathbb{G}) \cong L^{1}(\mathbb{G}) /\left\langle I \star L^{1}(\mathbb{G})\right\rangle$. Consider the commutative diagram:

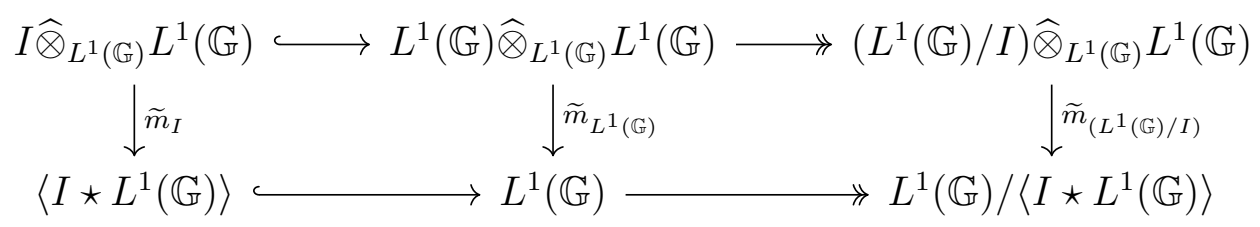

Since $\widehat{\mathbb{G}}$ is amenable, by Theorem 4.3.12 the top, and hence both rows are 1-exact. As the last two columns are completely isometric, it follows that $\widetilde{m}_{I}: I \widehat{\otimes}_{L^{1}(\mathbb{G})} L^{1}(\mathbb{G}) \cong$ $\left\langle I \star L^{1}(\mathbb{G})\right\rangle$ completely isometrically.

A locally compact quantum group $\mathbb{G}$ is said to be regular if

$$
\mathcal{K}\left(L^{2}(\mathbb{G})\right)=\left\langle(\mathrm{id} \otimes \omega)(\sigma V) \mid \omega \in \mathcal{T}\left(L^{2}(\mathbb{G})\right)\right\rangle,
$$

where $\mathcal{K}\left(L^{2}(\mathbb{G})\right)$ denotes the ideal of compact operators on $L^{2}(\mathbb{G})$, and as usual, $\langle\cdot\rangle$ denotes the closed linear span. For example, Kac algebras are regular, as well as discrete and compact quantum groups (see [51]). Under the assumption of regularity, we now obtain a version of Theorem 4.3.12 at the predual level.

Theorem 4.4.13. Let $\mathbb{G}$ be a locally compact quantum group. Consider the following conditions:

1. $\widehat{\mathbb{G}}$ is compact (equivalently, $\mathbb{G}$ is discrete);

2. $\mathcal{T}\left(L^{2}(\mathbb{G})\right)$ is relatively 1-projective in $\mathcal{T}_{\triangleright}-\bmod$;

3. $L^{1}(\mathbb{G})$ is 1-projective in $L^{1}(\mathbb{G})-\bmod$.

Then $(1) \Leftrightarrow(2) \Rightarrow(3)$, and when $\mathbb{G}$ is regular, the conditions are equivalent.

Proof. The implication (1) $\Rightarrow$ (2) follows by an argument similar to the proof of Theorem 4.3.12 using a normal two-sided invariant mean $\widehat{m}^{\prime}$ on $L^{\infty}\left(\widehat{\mathbb{G}}^{\prime}\right)$, which exists by compactness. The implication $(2) \Rightarrow(1)$ also follows similarly to Theorem 4.3.12, 
giving the relative 1-injectivity of $L^{\infty}(\mathbb{G})$ in $\operatorname{nmod}-L^{1}(\mathbb{G})$ and the existence of a normal conditional expectation $E: \mathcal{B}\left(L^{2}(\mathbb{G})\right) \rightarrow L^{\infty}(\mathbb{G})$. Hence, the inclusion $L^{\infty}(\mathbb{G}) \hookrightarrow \mathcal{B}\left(L^{2}(\mathbb{G})\right)$ is admissible in $\mathbf{n m o d}-L^{1}(\mathbb{G})$ with respect to the right $L^{1}(\mathbb{G})$ module structure on $\mathcal{B}\left(L^{2}(\mathbb{G})\right)$ given by (4.16). Relative 1-injectivity then implies the existence of a normal condition expectation $P: \mathcal{B}\left(L^{2}(\mathbb{G})\right) \rightarrow L^{\infty}(\mathbb{G})$ that is a right $L^{1}(\mathbb{G})$-module map under the $\triangleleft$-action. Thus, $\widehat{\mathbb{G}}$ is compact by Proposition 4.2.19.

$(2) \Rightarrow(3)$ : By the above we know that $\widehat{\mathbb{G}}$ is compact and $L^{\infty}(\mathbb{G})$ is relatively 1-injective in nmod $-L^{1}(\mathbb{G})$, which implies that $L^{1}(\mathbb{G})$ is relatively 1-projective in $L^{1}(\mathbb{G})$ - mod. By discreteness of $\mathbb{G}$ we have $L^{\infty}(\mathbb{G}) \cong \bigoplus\left\{M_{n_{\alpha}}(\mathbb{C}) \mid \alpha \in \operatorname{Irr}(\widehat{\mathbb{G}})\right\}$ which implies that $L^{1}(\mathbb{G}) \cong \bigoplus_{1}\left\{T_{n_{\alpha}}(\mathbb{C}) \mid \alpha \in \operatorname{Irr}(\widehat{\mathbb{G}})\right\}$, where $T_{n_{\alpha}}(\mathbb{C})$ is the space of $n_{\alpha} \times n_{\alpha}$ trace-class operators. Hence, $L^{1}(\mathbb{G})$ is 1 -projective in $\mathbb{C}-\bmod$ by $[8$, Proposition 3.6, Proposition 3.7]. The left version of Proposition 4.3.1 then entails the 1-projectivity of $L^{1}(\mathbb{G})$ in $L^{1}(\mathbb{G})-\bmod$.

Now, suppose that $\mathbb{G}$ is regular. Considering again the right $L^{1}(\mathbb{G})$-module structure on $\mathcal{B}\left(L^{2}(\mathbb{G})\right)$ given by the $\triangleleft$-action (4.16), it follows from [51, Corollary 3.6] that $\mathcal{K}\left(L^{2}(\mathbb{G})\right)$ is an essential $L^{1}(\mathbb{G})$-submodule of $\mathcal{B}\left(L^{2}(\mathbb{G})\right)$, that is, $\mathcal{K}\left(L^{2}(\mathbb{G})\right)=$ $\left\langle\mathcal{K}\left(L^{2}(\mathbb{G})\right) \triangleleft L^{1}(\mathbb{G})\right\rangle$. We show $(3) \Rightarrow(1)$.

Since the multiplication $m_{L^{1}(\mathbb{G})}: L^{1}(\mathbb{G}) \widehat{\otimes} L^{1}(\mathbb{G}) \rightarrow L^{1}(\mathbb{G})$ is a complete quotient morphism and $L^{1}(\mathbb{G})$ is 1 -projective in $L^{1}(\mathbb{G})-\bmod$, for every $\varepsilon>0$ there exists a morphism $\Phi_{\varepsilon}: L^{1}(\mathbb{G}) \rightarrow L^{1}(\mathbb{G}) \widehat{\otimes} L^{1}(\mathbb{G})$ satisfying $m_{L^{1}(\mathbb{G})} \circ \Phi_{\varepsilon}=\mathrm{id}_{L^{1}(\mathbb{G})}$ and $\left\|\Phi_{\varepsilon}\right\|_{c b}<$ $1+\varepsilon$. Moreover, we know that $L^{\infty}(\mathbb{G})$ is 1-injective in $\bmod -L^{1}(\mathbb{G})$ as the dual a 1projective module. Thus, $M_{c b}^{r}\left(L^{1}(\mathbb{G})\right) \cong C_{u}(\mathbb{G})^{*}$ by Proposition 4.4 .8 , and $L^{\infty}(\mathbb{G})$ is a 1-injective operator space. Appealing to [102, Theorem 3.1], there exits a net $\left(\Psi_{i}\right)_{i \in I}$ of normal finite-rank complete contractions $\Psi_{i}: L^{\infty}(\mathbb{G}) \rightarrow L^{\infty}(\mathbb{G})$ converging to $\mathrm{id}_{L^{\infty}(\mathbb{G})}$ in the point weak* topology. Using the normal completely bounded morphism $\Phi_{\varepsilon}^{*}: L^{\infty}(\mathbb{G}) \bar{\otimes} L^{\infty}(\mathbb{G}) \rightarrow L^{\infty}(\mathbb{G})$ which is a left inverse of $\Gamma$, one can argue in a similar manner to Theorem 4.4 .11 by averaging the normal finite-rank maps $\Psi_{i}$ into multipliers and use the fact that $L^{1}(\mathbb{G})$ is a two-sided ideal in $C_{u}(\mathbb{G})^{*}$ to obtain a 
bounded net $\left(f_{i}\right)_{i \in I}$ in $L^{1}(\mathbb{G})$ satisfying $f \star f_{i}-f \rightarrow 0$ weakly for all $f \in L^{1}(\mathbb{G})$. The standard convexity argument then yields a bounded right approximate identity for $L^{1}(\mathbb{G})$, and $\mathbb{G}$ is necessarily co-amenable.

Now, since $\pi:\left(\mathcal{T}\left(L^{2}(\mathbb{G})\right), \triangleleft\right) \rightarrow L^{1}(\mathbb{G})$ is a complete quotient morphism, for any $\varepsilon>0$ it also has a right inverse morphism $\Psi_{\varepsilon}: L^{1}(\mathbb{G}) \rightarrow \mathcal{T}\left(L^{2}(\mathbb{G})\right)$ with $\left\|\Psi_{\varepsilon}\right\|_{c b}<$ $1+\varepsilon$. Then $\Psi_{\varepsilon}^{*}: \mathcal{B}\left(L^{2}(\mathbb{G})\right) \rightarrow L^{\infty}(\mathbb{G})$ is a normal completely bounded right $\triangleleft$ module projection onto $L^{\infty}(\mathbb{G})$. Since $L^{1}(\mathbb{G})$ has a contractive approximate identity and $\mathcal{K}\left(L^{2}(\mathbb{G})\right)$ is an essential $L^{1}(\mathbb{G})$-module, we know that $\mathcal{K}\left(L^{2}(\mathbb{G})\right)$ is induced, that is, $\widetilde{m}_{\mathcal{K}\left(L^{2}(\mathbb{G})\right)}: \mathcal{K}\left(L^{2}(\mathbb{G})\right) \widehat{\otimes}_{L^{1}(\mathbb{G})} L^{1}(\mathbb{G}) \rightarrow \mathcal{K}\left(L^{2}(\mathbb{G})\right)$ is a completely isometric isomorphism. Hence, so too is its dual

$$
\left(\widetilde{m}_{\mathcal{K}\left(L^{2}(\mathbb{G})\right)}\right)^{*}: \mathcal{T}\left(L^{2}(\mathbb{G})\right) \cong \mathcal{C B}_{L^{1}(\mathbb{G})}\left(\mathcal{K}\left(L^{2}(\mathbb{G})\right), L^{\infty}(\mathbb{G})\right)
$$

Then $\left.\Psi_{\varepsilon}^{*}\right|_{\mathcal{K}\left(L^{2}(\mathbb{G})\right)} \in \mathcal{C B}_{L^{1}(\mathbb{G})}\left(\mathcal{K}\left(L^{2}(\mathbb{G})\right), L^{\infty}(\mathbb{G})\right)=\left(\widetilde{m}_{\mathcal{K}\left(L^{2}(\mathbb{G})\right)}\right)^{*}\left(\mathcal{T}\left(L^{2}(\mathbb{G})\right)\right)$, so let $\rho \in$ $\mathcal{T}\left(L^{2}(\mathbb{G})\right)$ satisfy $\left(\widetilde{m}_{\mathcal{K}\left(L^{2}(\mathbb{G})\right)}\right)^{*}(\rho)=\left.\Psi_{\varepsilon}^{*}\right|_{\mathcal{K}\left(L^{2}(\mathbb{G})\right)}$. Then for all $y \in \mathcal{K}\left(L^{2}(\mathbb{G})\right)$ and $f \in$ $L^{1}(\mathbb{G})$ we have

$$
\left\langle\left.\Psi_{\varepsilon}^{*}\right|_{\mathcal{K}\left(L^{2}(\mathbb{G})\right)}(y), f\right\rangle=\left\langle\left(\widetilde{m}_{\mathcal{K}\left(L^{2}(\mathbb{G})\right)}\right)^{*}(\rho)(y), f\right\rangle=\langle\rho, y \triangleleft f\rangle=\langle\rho \triangleleft y, f\rangle .
$$

By weak* density of $\mathcal{K}\left(L^{2}(\mathbb{G})\right)$ in $\mathcal{B}\left(L^{2}(\mathbb{G})\right)$, it follows that $\Psi_{\varepsilon}^{*}(T)=\rho \triangleleft T$ for all $T \in \mathcal{B}\left(L^{2}(\mathbb{G})\right)$. In particular,

$$
\pi(\rho) \star x=\rho \triangleleft x=\Psi_{\varepsilon}^{*}(x)=x
$$

for all $x \in L^{\infty}(\mathbb{G})$ as $\Psi_{\varepsilon}^{*}$ is a projection. Then $\pi(\rho)$ is a right identity for $L^{1}(\mathbb{G})$, and using the unitary antipode $R$ as in Theorem 4.4 .11 we may construct a two-sided identity for $L^{1}(\mathbb{G})$, that is, $\mathbb{G}$ is discrete, whence $\widehat{\mathbb{G}}$ is compact.

Analogously, there is a right module version of Theorem 4.4.13. 
Theorem 4.4.14. Let $\mathbb{G}$ be a locally compact quantum group. Consider the following conditions:

1. $\widehat{\mathbb{G}}$ is compact;

2. $\mathcal{T}\left(L^{2}(\mathbb{G})\right)$ is relatively 1-projective in $\bmod -\left(\mathcal{T}\left(L^{2}(\mathbb{G})\right), \triangleleft\right)$;

3. $L^{1}(\mathbb{G})$ is 1-projective in $\bmod -L^{1}(\mathbb{G})$.

Then $(1) \Leftrightarrow(2) \Rightarrow(3)$, and when $\mathbb{G}$ is regular, the conditions are equivalent.

Remark 4.4.15. As in Remark 4.3.11, it is not clear at this time whether we can replace 1-relative projectivity of $\mathcal{T}\left(L^{2}(\mathbb{G})\right)$ with 1-projectivity of $\mathcal{T}\left(L^{2}(\mathbb{G})\right)$ in the statements of Theorems 4.4.13 and 4.4.14. However, one cannot replace 1-projectivity of $L^{1}(\mathbb{G})$ with relative 1-projectivity of $L^{1}(\mathbb{G})$ in condition (3) of Theorems 4.4.13 and 4.4.14, as, for example, $L^{1}(G)$ is always relatively 1-projective for any locally compact group $G$ (see [18, Theorem 2.4]).

Since any co-commutative quantum group is regular, we immediately obtain a new characterization of compact groups.

Corollary 4.4.16. Let $G$ be a locally compact group. The following conditions are equivalent:

1. $G$ is compact;

2. $A(G)$ is 1-projective in $\bmod -A(G)$;

3. $A(G)$ is 1-projective in $A(G)-\bmod$. 


\section{Chapter 5}

\section{Inner Amenability and Relative Injectivity}

\subsection{Classical Theory}

If $G$ is a locally compact group and $p \in[1, \infty]$, then $G$ acts by conjugation on $L^{p}(G)$ via

$$
\beta_{p}(s) f(t)=f\left(s^{-1} t s\right) \Delta(s)^{1 / p}, \quad s \in G, f \in L^{p}(G) .
$$

When $p=2$, we obtain a strongly continuous unitary representation $\beta_{2}: G \rightarrow$ $\mathcal{B}\left(L^{2}(G)\right)$ satisfying $\beta_{2}(s)=\lambda(s) \rho(s)$ for $s \in G$, and when $p=\infty$, the conjugation action becomes

$$
\beta_{\infty}(s) f(t)=f\left(s^{-1} t s\right), \quad s \in G, f \in L^{\infty}(G) .
$$

Following Paterson $[82,2.35 . \mathrm{H}]$, we say that $G$ is inner amenable if there exists a state $m \in L^{\infty}(G)^{*}$ satisfying

$$
\left\langle m, \beta_{\infty}(s) f\right\rangle=\langle m, f\rangle \quad s \in G, f \in L^{\infty}(G) .
$$

Remark 5.1.1. In [27], Effros defined a discrete group $G$ to be "inner amenable" if there exists a conjugation invariant mean $m \in \ell^{\infty}(G)^{*}$ such that $m \neq \delta_{e}$. In what 
follows, inner amenability will always refer to the definition given above (5.1).

The class of inner amenable locally compact groups forms a large, interesting class of groups containing all amenable groups and IN groups, where a locally compact group $G$ is IN if there exists a compact neighborhood of the identity which is invariant under conjugation. If the identity has an entire neighborhood basis consisting of compact, conjugate invariant subsets, then $G$ is said to be SIN, which stands for small invariant neighborhoods. For example, compact, abelian and discrete groups are SIN.

A strongly continuous unitary representation $\pi: G \rightarrow \mathcal{B}\left(H_{\pi}\right)$ of a locally compact group $G$ is said to be amenable if there exists a state $m_{\pi} \in \mathcal{B}\left(H_{\pi}\right)^{*}$ such that

$$
\left\langle m_{\pi}, \pi(s) T \pi(s)^{*}\right\rangle=\left\langle m_{\pi}, T\right\rangle, \quad \forall s \in G, T \in \mathcal{B}\left(H_{\pi}\right) .
$$

This concept was introduced by Bekka [7], who showed, among other things, that $G$ is inner amenable precisely when $\beta_{2}$ is an amenable unitary representation [7, Theorem 2.4]. By [97, Proposition 3.1], inner amenability is equivalent to the existence of a $\beta_{2}$-invariant state on $\beta_{2}(G)^{\prime \prime} \subseteq \mathcal{B}\left(L^{2}(G)\right)$, the von Neumann subalgebra generated by the conjugate representation. We now show that inner amenability is equivalent to the existence of a $\beta_{2}$-invariant state on $\mathcal{L}(G)$, i.e., a $G$-invariant state under the canonical $G$-action on $\mathcal{L}(G)$ given by

$$
x \mapsto \lambda(s) x \lambda(s)^{*}, \quad x \in \mathcal{L}(G), s \in G .
$$

In turn, we answer a question raised by Lau and Paterson in [67, Example 5].

Proposition 5.1.2. A locally compact group $G$ is inner amenable if and only if there exists a $G$-invariant state on $\mathcal{L}(G)$.

Proof. If $G$ is inner amenable, then by [7, Theorem 2.4] there exists a $\beta_{2}$-invariant 
state $m \in \mathcal{B}\left(L^{2}(G)\right)^{*}$, whose restriction to $\mathcal{L}(G)$ is necessarily $G$-invariant, as

$$
\left\langle m, \lambda(s) x \lambda(s)^{*}\right\rangle=\left\langle m, \lambda(s) \rho(s) x \rho(s)^{*} \lambda(s)^{*}\right\rangle=\left\langle m, \beta_{2}(s) x \beta_{2}(s)^{*}\right\rangle=\langle m, x\rangle
$$

for all $x \in \mathcal{L}(G)$ and $s \in G$.

Conversely, suppose $m \in \mathcal{L}(G)^{*}$ is a $G$-invariant state. Since $\mathcal{L}(G)$ is standardly represented on $L^{2}(G)$, every normal state $\omega \in \mathcal{L}(G)_{*}$ is the restriction of a vector state $\omega_{\xi}$ to $\mathcal{L}(G)$ for a unique unit vector $\xi \in \mathcal{P}$, the closed cone generated by $f * J f$ [40, Lemma 2.10], where $f \in C_{c}(G)$, the continuous functions on $G$ with compact support, and $J f(s)=\overline{f\left(s^{-1}\right)} \Delta\left(s^{-1}\right)^{1 / 2}, s \in G$. Hence, there exists a net of unit vectors $\left(\xi_{\alpha}\right)_{\alpha \in A}$ in $\mathcal{P}$ such that $\left(\omega_{\xi_{\alpha}}\right)_{\alpha \in A}$ converges to $m$ in the weak* topology of $\mathcal{L}(G)^{*}$. By $G$-invariance, it follows that

$$
\beta_{2}(s) \cdot \omega_{\xi_{\alpha}} \cdot \beta_{2}(s)^{*}-\omega_{\xi_{\alpha}}=\omega_{\beta_{2}(s) \xi_{\alpha}}-\omega_{\xi_{\alpha}} \rightarrow 0
$$

weakly in $A(G)=\mathcal{L}(G)_{*}$ for all $s \in G$. By the standard convexity argument, it follows that there is a net of unit vectors $\left(\eta_{\gamma}\right)_{\gamma \in C}$ in $\mathcal{P}$ satisfying

$$
\left\|\beta_{2}(s) \cdot \omega_{\eta_{\gamma}} \cdot \beta_{2}(s)^{*}-\omega_{\eta_{\gamma}}\right\|_{A(G)}=\left\|\omega_{\beta_{2}(s) \eta_{\gamma}}-\omega_{\eta_{\gamma}}\right\|_{A(G)} \rightarrow 0, \quad s \in G .
$$

However, since $\beta_{2}(s)=\lambda(s) \rho(s)=\lambda(s) J \lambda(s) J$ we have $\beta_{2}(s) \mathcal{P} \subseteq \mathcal{P}$ for any $s \in G$ by (2.3). By [40, Lemma 2.10], we then have

$$
\left\|\beta_{2}(s) \eta_{\gamma}-\eta_{\gamma}\right\|_{L^{2}(G)}^{2} \leq\left\|\omega_{\beta_{2}(s) \eta_{\gamma}}-\omega_{\eta_{\gamma}}\right\|_{A(G)} \rightarrow 0, \quad s \in G .
$$

Since

$$
\left\|\beta_{2}(s)\left|\eta_{\gamma}\right|-\left|\eta_{\gamma}\right|\right\|_{L^{2}(G)} \leq\left\|\beta_{2}(s) \eta_{\gamma}-\eta_{\gamma}\right\|_{L^{2}(G)},
$$

we may assume without loss of generality that $\eta_{\gamma} \geq 0$. Then, by [40, Lemma 2.10]

$$
\left\|\omega_{\beta_{2}(s) \eta_{\gamma}}-\omega_{\eta_{\gamma}}\right\|_{L^{1}(G)} \leq 2\left\|\beta_{2}(s) \eta_{\gamma}-\eta_{\gamma}\right\|_{L^{2}(G)} \rightarrow 0
$$


for all $s \in G$, and letting $f_{\gamma}:=\eta_{\gamma}^{2}$, we obtain a net of states in $L^{1}(G)$ satisfying

$$
\left\|\beta_{1}(s) f_{\gamma}-f_{\gamma}\right\|_{L^{1}(G)}=\left\|\omega_{\beta_{2}(s) \eta_{\gamma}}-\omega_{\eta_{\gamma}}\right\|_{L^{1}(G)} \rightarrow 0, \quad s \in G .
$$

Any weak* cluster point $m \in L^{\infty}(G)^{*}$ of $\left(f_{\gamma}\right)_{\gamma \in C}$ will therefore be conjugate invariant, and $G$ is inner amenable.

As an immediate corollary, we obtain the following hereditary property of inner amenability which appears to be new.

Corollary 5.1.3. Let $G$ be a locally compact group and let $H$ be a closed subgroup of $G$. If $G$ is inner amenable, then $H$ is inner amenable.

Proof. Letting $\mathcal{L}_{H}(G):=\left\{\lambda_{G}(s) \mid s \in H\right\}^{\prime \prime} \subseteq \mathcal{L}(G)$, it follows that

$$
\Phi: \mathcal{L}(H) \ni \lambda_{H}(s) \mapsto \lambda_{G}(s) \in \mathcal{L}_{H}(G)
$$

is a *-isomorphism of von Neumann algebras. Thus, if $m \in \mathcal{L}(G)^{*}$ is a $G$-invariant state then $m_{H}:=\left.m\right|_{\mathcal{L}_{H}(G)} \circ \Phi \in \mathcal{L}(H)^{*}$ is an $H$-invariant state on $\mathcal{L}(H)$, so $H$ is inner amenable by Proposition 5.1.2.

Since a locally compact group $G$ is IN precisely when $\mathcal{L}(G)$ has a normal $G$ invariant state [103, Proposition 4.2], Proposition 5.1.2 shows that IN is to inner amenability what compactness is to amenability.

We now give a homological characterization of inner amenability.

Theorem 5.1.4. A locally compact group $G$ is inner amenable if and only if $\mathcal{L}(G)$ is relatively 1-injective in $A(G)-\bmod$.

Proof. If $G$ is inner amenable, then by [119, Theorem 1] there exists a net of unit vectors $\left(\xi_{\alpha}\right)_{\alpha \in A}$ in $L^{2}(G)$ satisfying

$$
\left\|\beta_{2}(s) \xi_{\alpha}-\xi_{\alpha}\right\|_{L^{2}(G)} \rightarrow 0
$$


uniformly on compact subsets of $G$. Thus, combining [91, Lemma 3.1, Lemma 4.1], it follows that $\Gamma_{s}: \mathcal{L}(G) \rightarrow \mathcal{L}(G) \bar{\otimes} \mathcal{L}(G)$ has a completely contractive left inverse $\Phi$ which is a left $A(G)$-module map. Since $\mathcal{L}(G)$ is faithful in $A(G)$ - mod, Proposition 4.3.3 implies that $\mathcal{L}(G)$ is relatively 1 -injective.

Conversely, relative 1-injectivity implies the existence of a completely contractive morphism $\Phi: \mathcal{L}(G) \bar{\otimes} \mathcal{L}(G) \rightarrow \mathcal{L}(G)$ satisfying $\Phi \circ \Gamma_{s}=\operatorname{id}_{\mathcal{L}(G)}$. It follows that $\Gamma_{s} \circ \Phi: \mathcal{L}(G) \bar{\otimes} \mathcal{L}(G) \rightarrow \mathcal{L}(G) \bar{\otimes} \mathcal{L}(G)$ is a projection of norm one onto the image of $\Gamma_{s}$, and therefore, as in the proof of Theorem 4.3.9,

$$
x \Phi(T) y=\Phi\left(\Gamma_{s}(x) T \Gamma_{s}(y)\right)
$$

for all $x, y \in \mathcal{L}(G)$ and $T \in \mathcal{L}(G) \bar{\otimes} \mathcal{L}(G)$.

Now, let $x \in \mathcal{L}(G)$. By the module property of $\Phi$ we have $\psi \cdot \Phi(x \otimes 1)=$ $\Phi(x \otimes \psi \cdot 1)=\psi(e) \Phi(x \otimes 1)$ for all $\psi \in A(G)$. The standard argument then gives $\Phi(x \otimes 1) \in \mathbb{C} 1$, so that $m: \mathcal{L}(G) \rightarrow \mathbb{C}$ defined by $\langle m, x\rangle=\Phi(x \otimes 1), x \in \mathcal{L}(G)$, yields a state on $\mathcal{L}(G)$. Moreover, by equation (5.2) we obtain

$$
\begin{aligned}
\left\langle m, \lambda(t) x \lambda(t)^{*}\right\rangle & =\Phi\left(\lambda(t) x \lambda(t)^{*} \otimes 1\right)=\Phi\left((\lambda(t) \otimes \lambda(t))(x \otimes 1)\left(\lambda(t)^{*} \otimes \lambda(t)^{*}\right)\right) \\
& =\Phi\left(\Gamma_{s}(\lambda(t))(x \otimes 1) \Gamma_{s}\left(\lambda(t)^{*}\right)\right)=\lambda(t) \Phi(x \otimes 1) \lambda(t)^{*}=\Phi(x \otimes 1) \\
& =\langle m, x\rangle
\end{aligned}
$$

for any $x \in \mathcal{L}(G)$ and $t \in G$. Thus, $m$ is a $G$-invariant state on $\mathcal{L}(G)$, which by Proposition 5.1.2 implies that $G$ is inner amenable.

In [91, Lemma 3.2] Ruan and $\mathrm{Xu}$ implicity showed that $A(G)$ is relatively 1projective as an operator module over itself whenever $G$ is an IN group (see also [33, Theorem 4.1]). We can now establish the converse, providing a partial solution to the open question of relative $C$-projectivity of $A(G)[33, \S 4]$. 
Corollary 5.1.5. Let $G$ be a locally compact group. Then $A(G)$ is relatively 1projective in $\bmod -A(G)$ if and only if $G$ is an IN group.

Proof. Assuming relative 1-projectivity of $A(G)$, there exists a normal completely contractive left $A(G)$-module map $\Phi: \mathcal{L}(G) \bar{\otimes} \mathcal{L}(G) \rightarrow \mathcal{L}(G)$ such that $\Phi \circ \Gamma_{s}=\mathrm{id}_{\mathcal{L}(G)}$. By the proof of Theorem 5.1.4 we obtain a normal $G$-invariant state on $\mathcal{L}(G)$, which, by [103, Proposition 4.2], implies that $G$ is IN. The converse follows from [91, Lemma $3.2]$.

Not surprisingly, there is a manifestation of inner amenability at the level of $\mathcal{T}\left(L^{2}(G)\right)$. The added advantage, however, is that it leads naturally to a notion of inner amenability for locally compact quantum groups.

Proposition 5.1.6. A locally compact group $G$ is inner amenable if and only if there exists a state $m \in \mathcal{B}\left(L^{2}(G)\right)^{*}$ such that

$$
\left\langle m, \rho \triangleright_{a} T\right\rangle=\left\langle m, T \triangleleft_{a} \rho\right\rangle, \quad \rho \in \mathcal{T}\left(L^{2}(G)\right), T \in \mathcal{B}\left(L^{2}(G)\right) .
$$

Proof. If $G$ is inner amenable, then by [119, Theorem 1] there exists a net of unit vectors $\left(\xi_{\alpha}\right)_{\alpha \in A}$ in $L^{2}(G)$ satisfying

$$
\left\|\lambda(s) \xi_{\alpha}-\rho(s)^{*} \xi_{\alpha}\right\|_{L^{2}(G)}=\left\|\beta_{2}(s) \xi_{\alpha}-\xi_{\alpha}\right\|_{L^{2}(G)} \rightarrow 0
$$

uniformly on compact subsets of $G$. Passing to a subnet, we may assume that the net $\left(\omega_{\xi_{\alpha}}\right)$ of normal states on $\mathcal{B}\left(L^{2}(G)\right)$ converges weak* to some state $m \in \mathcal{B}\left(L^{2}(G)\right)^{*}$. Then, for $\rho \in \mathcal{T}\left(L^{2}(G)\right), T \in \mathcal{B}\left(L^{2}(G)\right)$, we have

$$
\begin{aligned}
\left\langle m, \rho \triangleright_{a} T\right\rangle & =\lim _{\alpha}\left\langle\omega_{\xi_{\alpha}}, \rho \triangleright_{a} T\right\rangle=\lim _{\alpha}\left\langle T \triangleright_{a} \omega_{\xi_{\alpha}}, \rho\right\rangle \\
& =\lim _{\alpha} \int_{G}\left\langle\rho(s) T \rho(s)^{*} \xi_{\alpha}, \xi_{\alpha}\right\rangle \pi(\rho)(s) d s \\
& =\lim _{\alpha} \int_{G}\left\langle T \rho(s)^{*} \xi_{\alpha}, \rho(s)^{*} \xi_{\alpha}\right\rangle \pi(\rho)(s) d s .
\end{aligned}
$$


Using the compact convergence (5.4), it follows that

$$
\begin{aligned}
\lim _{\alpha} \int_{G}\left\langle T \rho(s)^{*} \xi_{\alpha}, \rho(s)^{*} \xi_{\alpha}\right\rangle \pi(\rho)(s) d s & =\lim _{\alpha} \int_{G}\left\langle T \lambda(s) \xi_{\alpha}, \lambda(s) \xi_{\alpha}\right\rangle \pi(\rho)(s) d s \\
& =\lim _{\alpha} \int_{G}\left\langle\lambda(s)^{*} T \lambda(s) \xi_{\alpha}, \xi_{\alpha}\right\rangle \pi(\rho)(s) d s \\
& =\lim _{\alpha}\left\langle\omega_{\xi_{\alpha}} \triangleleft_{a} T, \rho\right\rangle \\
& =\lim _{\alpha}\left\langle\omega_{\xi_{\alpha}}, T \triangleleft_{a} \rho\right\rangle \\
& =\left\langle m, T \triangleleft_{a} \rho\right\rangle .
\end{aligned}
$$

Thus, $m$ satisfies (5.3).

Conversely, if such a state exists, then its restriction to $\mathcal{L}(G)$ satisfies

$$
\left\langle m, x \triangleleft_{a} \rho\right\rangle=\left\langle m, \rho \triangleright_{a} x\right\rangle=\langle\rho, 1\rangle\langle m, x\rangle
$$

for all $x \in \mathcal{L}(G)$ and $\rho \in \mathcal{T}\left(L^{2}(G)\right)$. In particular, $\langle m, x\rangle=\left\langle m, x \triangleleft_{a} \rho\right\rangle$ for all states $\rho \in \mathcal{T}\left(L^{2}(G)\right)$. Let $t \in G$ and $h \in L^{\infty}(G)$. Then

$$
\begin{aligned}
\left\langle\pi\left(\lambda(t)^{*} \rho \lambda(t)\right), h\right\rangle & =\left\langle\lambda(t)^{*} \rho \lambda(t), M_{h}\right\rangle=\left\langle\rho, \lambda(t) M_{h} \lambda(t)^{*}\right\rangle=\left\langle\rho, M_{\lambda(t) h}\right\rangle \\
& =\langle\pi(\rho), \lambda(t) h\rangle=\left\langle\lambda\left(t^{-1}\right) \pi(\rho), h\right\rangle .
\end{aligned}
$$

Hence, $\pi\left(\lambda(t)^{*} \rho \lambda(t)\right)(s)=\pi(\rho)(t s)$ for all $s, t \in G$. Then

$$
\begin{aligned}
\left(\lambda(t) x \lambda(t)^{*}\right) \triangleleft_{a} \rho & =\int_{G} \lambda\left(s^{-1} t\right) x \lambda\left(t^{-1} s\right) \pi(\rho)(s) d s \\
& =\int_{G} \lambda(r)^{*} x \lambda(r) \pi(\rho)(t r) d r \\
& =\int_{G} \lambda(r)^{*} x \lambda(r) \pi\left(\lambda(t)^{*} \rho \lambda(t)\right)(r) d r \\
& =x \triangleleft_{a}\left(\lambda(t)^{*} \rho \lambda(t)\right),
\end{aligned}
$$


so that

$$
\left\langle m, \lambda(t) x \lambda(t)^{*}\right\rangle=\left\langle m,\left(\lambda(t) x \lambda(t)^{*}\right) \triangleleft_{a} \rho\right\rangle=\left\langle m, x \triangleleft_{a}\left(\lambda(t)^{*} \rho \lambda(t)\right)\right\rangle=\langle m, x\rangle
$$

for all $x \in \mathcal{L}(G), t \in G$ and states $\rho \in \mathcal{T}\left(L^{2}(G)\right)$. Thus, $G$ is inner amenable by Proposition 5.1.2.

\subsection{Quantum Theory}

In [35], a notion of inner amenability was given for an arbitrary locally compact quantum group $\mathbb{G}$ by means of the existence of a state $m \in L^{\infty}(\mathbb{G})^{*}$ satisfying

$$
\langle m, x \star f\rangle=\langle m, f \star x\rangle, \quad f \in L^{1}(\mathbb{G}), x \in L^{\infty}(\mathbb{G}) .
$$

In other words, there exits a "tracial" state for the bimodule action of $L^{1}(\mathbb{G})$ on $L^{\infty}(\mathbb{G})$. However, if $\mathbb{G}$ is any co-amenable locally compact quantum group, then any cluster point in $L^{\infty}(\mathbb{G})^{*}$ of a contractive approximate identity in $L^{1}(\mathbb{G})$ is such a tracial state. In particular, any locally compact group gives rise to an "inner amenable" locally compact quantum group in the sense of [35].

In this section we introduce a suitable notion of inner amenability for locally compact quantum groups. Indeed, we will show that $\mathbb{G}_{a}$ is inner amenable precisely when the underlying locally compact group $G$ is inner amenable. We begin a systematic study of its basic properties, provide examples, and discuss related notions. In particular, we investigate its relationship with relative injectivity. 


\subsubsection{Definition and Basic Properties}

Definition 5.2.1. Let $\mathbb{G}$ be a locally compact quantum group. Then we call $\mathbb{G}$ inner amenable if there exists a state $m \in \mathcal{B}\left(L^{2}(\mathbb{G})\right)^{*}$ satisfying

$$
\langle m, \rho \triangleright T\rangle=\langle m, T \triangleleft \rho\rangle, \quad \rho \in \mathcal{T}\left(L^{2}(\mathbb{G})\right), T \in \mathcal{B}\left(L^{2}(\mathbb{G})\right) .
$$

Such a state $m$ is said to be an inner invariant mean.

By Proposition 5.1.6, a commutative quantum group $\mathbb{G}_{a}$ is inner amenable if and only if its underlying locally compact group $G$ is inner amenable, so Definition 5.2.1 is a bona fide generalization of classical inner amenability.

Remark 5.2.2. A natural question is to seek a definition of inner amenability at the level of $L^{\infty}(\mathbb{G})$. Motivated by the group case, one is led to consider the conjugate co-representation $W \sigma V \sigma=W(1 \otimes U) W\left(1 \otimes U^{*}\right) \in L^{\infty}(\mathbb{G}) \bar{\otimes} \mathcal{B}\left(L^{2}(\mathbb{G})\right)$ of $\mathbb{G}$ (see [90]), and the associated map $\widetilde{\beta_{2}}: \mathcal{B}\left(L^{2}(\mathbb{G})\right) \rightarrow L^{\infty}(\mathbb{G}) \bar{\otimes} \mathcal{B}\left(L^{2}(\mathbb{G})\right)$ defined by

$$
\widetilde{\beta_{2}}(T)=(W \sigma V \sigma)^{*}(1 \otimes T) W \sigma V \sigma, \quad T \in \mathcal{B}\left(L^{2}(\mathbb{G})\right) .
$$

If $\widetilde{\beta}_{2}\left(L^{\infty}(\mathbb{G})\right) \subseteq L^{\infty}(\mathbb{G}) \bar{\otimes} L^{\infty}(\mathbb{G})$, i.e., it leaves $L^{\infty}(\mathbb{G})$ globally invariant, then one could define a corresponding conjugation action on $L^{\infty}(\mathbb{G})$. This invariance, however, is not clear beyond the commutative case.

Following [78], we say that a locally compact quantum group $\mathbb{G}$ is strongly inner amenable if there exists a net $\left(\xi_{\alpha}\right)_{\alpha \in A}$ of unit vectors such that

$$
\left\|W \sigma V \sigma\left(\eta \otimes \xi_{\alpha}\right)-\left(\eta \otimes \xi_{\alpha}\right)\right\| \rightarrow 0, \quad \eta \in L^{2}(\mathbb{G}) .
$$

Proposition 5.2.3. Let $\mathbb{G}$ be a locally compact quantum group. If $\mathbb{G}$ is strongly inner amenable then it is inner amenable.

Proof. Let $\left(\xi_{\alpha}\right)_{\alpha \in A}$ be a net of unit vectors asymptotically invariant under the conju- 
gate co-representation. Passing to a subnet, we may assume that $\left(\omega_{J \xi_{\alpha}}\right)_{\alpha \in A}$ converges weak* $^{*}$ to a state $m \in \mathcal{B}\left(L^{2}(\mathbb{G})\right)^{*}$. For any $\eta \in L^{2}(\mathbb{G})$ and $T \in \mathcal{B}\left(L^{2}(\mathbb{G})\right)$, using equation (3.5) together with the adjoint relations of $W$ and $V$, we have

$$
\begin{aligned}
\left\langle m, \omega_{\eta} \triangleright T\right\rangle & =\lim _{\alpha}\left\langle\omega_{J \xi_{\alpha}}, \omega_{\eta} \triangleright T\right\rangle=\lim _{\alpha}\left\langle V(T \otimes 1) V^{*}\left(J \xi_{\alpha} \otimes \eta\right),\left(J \xi_{\alpha} \otimes \eta\right)\right\rangle \\
& =\lim _{\alpha}\left\langle(1 \otimes T) \sigma V^{*} \sigma\left(\eta \otimes J \xi_{\alpha}\right), \sigma V^{*} \sigma\left(\eta \otimes J \xi_{\alpha}\right)\right\rangle \\
& =\lim _{\alpha}\left\langle(1 \otimes T)(\widehat{J} \otimes J) \sigma V \sigma\left(\widehat{J} \eta \otimes \xi_{\alpha}\right),(\widehat{J} \otimes J) \sigma V \sigma\left(\widehat{J} \eta \otimes \xi_{\alpha}\right)\right\rangle \\
& =\lim _{\alpha}\left\langle(1 \otimes T)(\widehat{J} \otimes J) W^{*}\left(\widehat{J} \eta \otimes \xi_{\alpha}\right),(\widehat{J} \otimes J) W^{*}\left(\widehat{J} \eta \otimes \xi_{\alpha}\right)\right\rangle \\
& =\lim _{\alpha}\left\langle(1 \otimes T) W\left(\eta \otimes J \xi_{\alpha}\right), W\left(\eta \otimes J \xi_{\alpha}\right)\right\rangle \\
& =\lim _{\alpha}\left\langle W^{*}(1 \otimes T) W\left(\eta \otimes J \xi_{\alpha}\right),\left(\eta \otimes J \xi_{\alpha}\right)\right\rangle \\
& =\lim _{\alpha}\left\langle\omega_{J \xi_{\alpha}}, T \triangleleft \omega_{\eta}\right\rangle \\
& =\left\langle m, T \triangleleft \omega_{\eta}\right\rangle .
\end{aligned}
$$

By linearity and density of $\left\{\omega_{\eta} \mid \eta \in L^{2}(\mathbb{G})\right\}$ in $\mathcal{T}\left(L^{2}(\mathbb{G})\right)$, it follows that $m$ is an inner invariant mean.

In the group case, strong inner amenability coincides with inner amenability by [119, Theorem 1]. It is not clear, however, if the equivalence persists for general quantum groups.

If $\mathbb{G}$ is a compact quantum group, then it is easy to see that $\Lambda_{\varphi}(1) \in L^{2}(\mathbb{G})$ is invariant under the conjugate co-representation $W \sigma V \sigma$, and hence, $\mathbb{G}$ is strongly inner amenable. More generally, any amenable quantum group is inner amenable.

Proposition 5.2.4. Let $\mathbb{G}$ be a locally compact quantum group. If $\mathbb{G}$ is amenable then it is inner amenable.

Proof. Let $m \in L^{\infty}(\mathbb{G})^{*}$ be a two-sided invariant mean. Then, denoting again by $m$ its restriction to $\operatorname{LUC}(\mathbb{G})$, let $n:=\rho_{0} \circ \Theta^{r}(m) \in \mathcal{B}\left(L^{2}(\mathbb{G})\right)^{*}$, where $\rho_{0} \in \mathcal{T}\left(L^{2}(\mathbb{G})\right)$ 
is a fixed normal state, and consider the state $R^{*}(n) \square n \in \mathcal{B}\left(L^{2}(\mathbb{G})\right)^{*}$ where $R$ is the extended unitary antipode and $\square$ is the left Arens product on $\mathcal{B}\left(L^{2}(\mathbb{G})\right)^{*}$ lifting the multiplication in $\left(\mathcal{T}\left(L^{2}(\mathbb{G})\right), \triangleright\right)$.

Fix $\rho, \omega \in \mathcal{T}\left(L^{2}(\mathbb{G})\right)$ and $T \in \mathcal{B}\left(L^{2}(\mathbb{G})\right)$. Firstly, we have

$$
\begin{aligned}
\langle n \square \rho, T\rangle & =\langle n, \rho \triangleright T\rangle=\left\langle\rho_{0}, \Theta^{r}(m) \circ \Theta^{r}(\pi(\rho))(T)\right\rangle \\
& =\left\langle\rho_{0}, \Theta^{r}(m \square \pi(\rho))(T)\right\rangle=\langle\rho, 1\rangle\langle n, T\rangle .
\end{aligned}
$$

Hence, $n \square \rho=\langle\rho, 1\rangle n$. Secondly, since $\Theta^{r}(m)$ is a right $\mathcal{T}_{\triangleright}$-covariant conditional expectation onto $L^{\infty}(\widehat{\mathbb{G}})$, we also have

$$
\begin{aligned}
\langle n \square(T \triangleleft \rho), \omega\rangle & =\langle n,(T \triangleleft \rho) \triangleright \omega\rangle \\
& =\left\langle\rho_{0}, \Theta^{r}(m)((T \triangleleft \rho) \triangleright \omega)\right\rangle \\
& =\left\langle\rho_{0}, \Theta^{r}(m)(T \triangleleft \rho) \triangleright \omega\right\rangle \\
& =\left\langle\rho_{0}, 1\right\rangle\left\langle\Theta^{r}(m)(T \triangleleft \rho), \omega\right\rangle \\
& =\left\langle\rho_{0}, 1\right\rangle\left\langle\Theta^{r}(m)(T) \triangleleft \rho, \omega\right\rangle \quad \text { (by Proposition 4.2.10) } \\
& =\left\langle\rho_{0}, 1\right\rangle\left\langle\Theta^{r}(m)(T), \rho \triangleleft \omega\right\rangle \\
& =\left\langle\rho_{0}, \Theta^{r}(m)(T) \triangleright(\rho \triangleleft \omega)\right\rangle \\
& =\left\langle\rho_{0}, \Theta^{r}(m)(T \triangleright(\rho \triangleleft \omega))\right\rangle \\
& =\langle n, T \triangleright(\rho \triangleleft \omega)\rangle \\
& =\langle n \square T, \rho \triangleleft \omega\rangle \\
& =\langle(n \square T) \triangleleft \rho, \omega\rangle .
\end{aligned}
$$

Thus, $n \square(T \triangleleft \rho)=(n \square T) \triangleleft \rho$. Putting things together, on the one hand we obtain

$$
\left\langle R^{*}(n) \square n, \rho \triangleright T\right\rangle=\left\langle R^{*}(n),(n \square \rho) \square T\right\rangle=\langle\rho, 1\rangle\left\langle R^{*}(n), n \square T\right\rangle,
$$


and on the other,

$$
\begin{aligned}
\left\langle R^{*}(n) \square n, T \triangleleft \rho\right\rangle & =\left\langle R^{*}(n), n \square(T \triangleleft \rho)\right\rangle=\left\langle R^{*}(n),(n \square T) \triangleleft \rho\right\rangle \\
& =\langle n, R((n \square T) \triangleleft \rho)\rangle=\left\langle n, R_{*}(\rho) \triangleright R(n \square T)\right\rangle \\
& =\left\langle n \square R_{*}(\rho), R(n \square T)\right\rangle=\left\langle R_{*}(\rho), 1\right\rangle\langle n, R(n \square T)\rangle \\
& =\langle\rho, 1\rangle\left\langle R^{*}(n), n \square T\right\rangle .
\end{aligned}
$$

Therefore, $R^{*}(n) \square n$ is the required inner invariant mean.

Combining Corollary 4.2.12 with Proposition 5.2.4, we obtain a quantum group analogue of the Lau-Paterson result in [66, Corollary 3.2], which states that a locally compact group $G$ is amenable if and only if it is inner amenable and $\mathcal{L}(G)$ is 1-injective $\mathbb{C}-\bmod$.

Corollary 5.2.5. A locally compact quantum group $\mathbb{G}$ is amenable if and only if it is inner amenable and $L^{\infty}(\widehat{\mathbb{G}})$ is 1-injective in $\mathbb{C}-\bmod$.

Motivated by the group setting, we propose the following definition of IN for quantum groups.

Definition 5.2.6. Let $\mathbb{G}$ be a locally compact quantum group. We say that $\mathbb{G}$ is IN if there is a normal state $\omega \in \mathcal{T}\left(L^{2}(\mathbb{G})\right)$ satisfying

$$
\langle\omega, \rho \triangleright T\rangle=\langle\omega, T \triangleleft \rho\rangle, \quad \rho \in \mathcal{T}\left(L^{2}(\mathbb{G})\right), T \in \mathcal{B}\left(L^{2}(\mathbb{G})\right) .
$$

Note that $\omega \in \mathcal{T}\left(L^{2}(\mathbb{G})\right)$ satisfies (5.6) if and only if

$$
\omega \triangleright \rho=\rho \triangleleft \omega, \quad \rho \in \mathcal{T}\left(L^{2}(\mathbb{G})\right),
$$

meaning that $\omega$ is a central element of $\mathcal{T}\left(L^{2}(\mathbb{G})\right)$ with respect to the $\mathcal{T}_{\triangleleft}-\mathcal{T}_{\triangleright}$-bimodule structure. This suggests that (5.7) is the appropriate notion of centrality in $\mathcal{T}\left(L^{2}(\mathbb{G})\right)$. 
Indeed, the next proposition shows that the center $\mathcal{Z}\left(\mathcal{T}_{\triangleright}\right)=\{0\}$ for any non-trivial quantum group $\mathbb{G}$.

Proposition 5.2.7. Let $\mathbb{G}$ be a locally compact quantum group. If $\mathcal{Z}\left(\mathcal{T}_{\triangleright}\right) \neq\{0\}$, then $\mathbb{G}=\mathbb{C} 1$ is trivial.

Proof. Let $\omega \in \mathcal{Z}\left(\mathcal{T}_{\triangleright}\right)$ be non-zero. Then $\omega \triangleright T=T \triangleright \omega$ for all $T \in \mathcal{B}\left(L^{2}(\mathbb{G})\right)$, which, by the commutation relation (4.4), means that for any state $\tau \in \mathcal{T}\left(L^{2}(\mathbb{G})\right)$,

$$
\begin{aligned}
T \triangleright \omega & =\tau \widehat{\triangleright}(T \triangleright \omega)=\tau \widehat{\triangleright}(\omega \triangleright T)=\omega \triangleright(\tau \widehat{\triangleright} T) \\
& =(\tau \widehat{\triangleright} T) \triangleright \omega=T \triangleright(\omega \widehat{\triangleright} \tau) .
\end{aligned}
$$

It follows that $\omega$ and $\omega \widehat{\triangleright} \tau$ agree on the weak* dense subspace $\left\langle\mathcal{T}\left(L^{2}(\mathbb{G})\right) \triangleright \mathcal{B}\left(L^{2}(\mathbb{G})\right)\right\rangle$ of $\mathcal{B}\left(L^{2}(\mathbb{G})\right)$, and therefore, by normality, $\omega=\omega \widehat{\triangleright} \tau$. By linearity, we obtain the equality $\omega=\omega \widehat{\triangleright} \tau$ for any $\tau \in \mathcal{T}\left(L^{2}(\mathbb{G})\right)$. Thus, $\omega$ is a left identity for $\widehat{\triangleright}$ which implies that $\widehat{\mathbb{G}}$, and therefore $\mathbb{G}$, is trivial [56, Proposition 3.7].

By the argument of Proposition 5.2.4 it immediately follows that any compact quantum group is IN. Also, any co-commutative quantum group $\mathbb{G}_{s}$ is IN. Indeed, using (3.5) we have

$$
V_{s}=\sigma(1 \otimes U) W_{s}\left(1 \otimes U^{*}\right) \sigma=\sigma(1 \otimes U) \sigma W_{a}^{*} \sigma\left(1 \otimes U^{*}\right) \sigma=(U \otimes 1) W_{a}^{*}(U \otimes 1),
$$

and by equation (3.2),

$$
\begin{aligned}
(U \otimes 1) W_{a}^{*}(U \otimes 1) \xi(s, t) & =W_{a}^{*}(U \otimes 1) \xi\left(s^{-1}, t\right) \Delta(s)^{-1 / 2} \\
& =(U \otimes 1) \xi\left(s^{-1}, s^{-1} t\right) \Delta(s)^{-1 / 2} \\
& =\xi\left(s, s^{-1} t\right) \\
& =W_{a} \xi(s, t)
\end{aligned}
$$

for all $s, t \in G$ and $\xi \in L^{2}(G \times G)$. Hence, $V_{s}=W_{a}$, that is, the right fundamental 
unitary of $\mathbb{G}_{s}$ is the left fundamental unitary of $\mathbb{G}_{a}$. Thus, for any $\rho, \omega \in \mathcal{T}\left(L^{2}(\mathbb{G})\right)$ and $T \in \mathcal{B}\left(L^{2}(\mathbb{G})\right)$ we have

$$
\begin{aligned}
\left\langle\omega \triangleright_{s} \rho, T\right\rangle & =\left\langle\omega,(\operatorname{id} \otimes \rho)\left(V_{s}(T \otimes 1) V_{s}^{*}\right)\right\rangle \\
& =\left\langle\omega,(\operatorname{id} \otimes \rho)\left(W_{a}(T \otimes 1) W_{a}^{*}\right\rangle\right. \\
& =\left\langle\omega,(\rho \otimes \mathrm{id})\left(W_{s}^{*}(1 \otimes T) W_{s}\right)\right\rangle \\
& =\left\langle\rho \triangleleft_{s} \omega, T\right\rangle,
\end{aligned}
$$

implying that every $\omega \in \mathcal{T}\left(L^{2}(\mathbb{G})\right)$ is a normal inner invariant mean for $\mathbb{G}_{s}$.

To further elucidate our definition of IN for quantum groups, we now derive a sufficient criterion.

Proposition 5.2.8. Let $\mathbb{G}$ be a locally compact quantum group. If there exists a non-zero vector $\xi \in L^{2}(\mathbb{G})$ satisfying

$$
\lambda(f) \xi=\widehat{J} \widehat{\tau}_{-i / 2}\left(\lambda(f)^{*}\right) \widehat{J} \xi, \quad f \in L^{1}(\mathbb{G}),
$$

then $\mathbb{G}$ is IN. When $\mathbb{G}=\mathbb{G}_{a}$ is commutative, the converse holds.

Proof. Let $\eta \in L^{2}(\mathbb{G})$. Resolving the identity $\operatorname{id}_{L^{2}(\mathbb{G})}$ with respect to an orthonormal basis $\left(e_{i}\right)_{i \in I}$ of $L^{2}(\mathbb{G})$, we have

$$
\omega_{\eta} \triangleright T=\left(\mathrm{id} \otimes \omega_{\eta}\right) V(T \otimes 1) V^{*}=\sum_{i \in I} V_{i} T V_{i}^{*}
$$

and

$$
T \triangleleft \omega_{\eta}=\left(\omega_{\eta} \otimes \mathrm{id}\right) W^{*}(1 \otimes T) W=\sum_{i \in I} W_{i}^{*} T W_{i}
$$

where $V_{i}=\left(\operatorname{id} \otimes \omega_{e_{i}, \eta}\right)(V), W_{i}=\left(\omega_{\eta, e_{i}} \otimes\right.$ id $)(W)=\lambda\left(\left.\omega_{\eta, e_{i}}\right|_{L^{\infty}(\mathbb{G})}\right)$ and both sums converge in the weak* topology. It suffices to show that $\xi \in L^{2}(\mathbb{G})$ satisfies $V_{i}^{*} \xi=$ $W_{i} \xi$ for all $i \in I$ and all $\eta \in L^{1}(\mathbb{G})$, as the corresponding normalized vector state $\omega_{\widetilde{\xi}} \in \mathcal{T}\left(L^{2}(\mathbb{G})\right)$ with $\widetilde{\xi}:=\xi /\|\xi\|$, would be a normal inner invariant mean for $\mathbb{G}$. To 
this end, we use the fact that $V=(U \otimes 1) \widehat{W}\left(U^{*} \otimes 1\right)$ (see (3.5)) to obtain

$$
\begin{aligned}
V_{i}^{*} & =\left(\mathrm{id} \otimes \omega_{e_{i}, \eta}\right)(V)^{*}=\left(\mathrm{id} \otimes \omega_{\eta, e_{i}}\right)\left(V^{*}\right)=U\left(\mathrm{id} \otimes \omega_{\eta, e_{i}}\right)(\widehat{W}) U^{*} \\
& =U\left(\omega_{\eta, e_{i}} \otimes \mathrm{id}\right)\left(W^{*}\right) U^{*}=U \widehat{S}^{-1}\left(\left(\omega_{\eta, e_{i}} \otimes \mathrm{id}\right)(W)\right) U^{*}=U \widehat{S}^{-1}\left(\lambda\left(\omega_{\eta, e_{i}}\right)\right) U^{*} \\
& =\widehat{J} J \widehat{R}\left(\widehat{\tau}_{i / 2}\left(\lambda\left(\omega_{\eta, e_{i}}\right)\right)\right) J \widehat{J}=\widehat{J}_{\widehat{\tau}_{-i / 2}}\left(\lambda\left(\omega_{\eta, e_{i}}\right)^{*}\right) \widehat{J} .
\end{aligned}
$$

As $W_{i}=\lambda\left(\left.\omega_{\eta, e_{i}}\right|_{L^{\infty}(\mathbb{G})}\right)$, and $\xi$ satisfies $(5.8)$, we have

$$
V_{i}^{*} \xi=\widehat{J} \widehat{\tau}_{-i / 2}\left(\lambda\left(\left.\omega_{\eta, e_{i}}\right|_{L^{\infty}(\mathbb{G})}\right)^{*}\right) \widehat{J} \xi=W_{i} \xi
$$

for all $i \in I$ and $\eta \in L^{2}(\mathbb{G})$.

Now, suppose that $\mathbb{G}=\mathbb{G}_{a}$ is commutative. The relation (5.8) then becomes

$$
\lambda(f) \xi=\rho\left(f^{o}\right) \xi, \quad f \in L^{1}(G)
$$

where $f^{o}(s)=\overline{f\left(s^{-1}\right)} \Delta\left(s^{-1}\right)$ is the usual involution on $L^{1}(G)$. For $s \in G$, let $\delta_{s}$ be the corresponding point mass in $M(G)$. If $\left(f_{i}\right)_{i \in I}$ is a contractive approximate identity in $L^{1}(G)$ then the net $\left(f_{i}^{s}\right)_{i \in I}$ given by $f_{i}^{s}=\delta_{s} * f_{i}$ satisfies the norm convergence

$$
f_{i}^{s} * g \rightarrow \delta_{s} * g \quad \text { and } \quad g * f_{i}^{s} \rightarrow g * \delta_{s}, \quad g \in L^{1}(G)
$$

Since $L^{2}(G)$ is an essential $L^{1}(G)$-module under the action $f * \eta=\lambda(f) \eta, f \in L^{1}(G)$, $\eta \in L^{2}(G)$, by Cohen's factorization theorem we have $L^{2}(G)=L^{1}(G) * L^{2}(G)$. Hence, $\xi=g * \eta$ for some $g \in L^{1}(G)$ and $\eta \in L^{2}(G)$. Then

$$
\lambda(s) \xi=\lambda(s) g * \eta=\lambda\left(\delta_{s} * g\right) \eta=\lim _{i \in I} \lambda\left(f_{i}^{s} * g\right) \eta=\lim _{i \in I} \lambda\left(f_{i}^{s}\right) \xi
$$

On the other hand, $L^{2}(G)$ is also an essential $L^{1}(G)$-module under the action $f \cdot \eta=$ $\rho(f) \eta, f \in L^{1}(G), \eta \in L^{2}(G)$, so again by Cohen's factorization theorem we have $\xi=h \cdot \beta$ for some $h \in L^{1}(G)$ and $\beta \in L^{2}(G)$. Then, by continuity of the involution 
on $L^{1}(G)$ we have

$$
\rho\left(s^{-1}\right) \xi=\rho\left(s^{-1}\right) h * \beta=\rho\left(\delta_{s^{-1}} * h\right) \beta=\lim _{i \in I} \rho\left(\left(f_{i}^{s}\right)^{o} * h\right) \beta=\lim _{i \in I} \rho\left(\left(f_{i}^{s}\right)^{o}\right) \xi .
$$

Thus, by equation (5.9) we have $\lambda(s) \xi=\rho\left(s^{-1}\right) \xi$. Since $s \in G$ was arbitrary, it follows that $\left.\omega_{\xi}\right|_{\mathcal{L}(G)}$ is a normal $G$-invariant state on $\mathcal{L}(G)$, so $G$ is IN by [103, Proposition $4.2]$.

To further elucidate equation (5.8), let us suppose that $\xi$ in Proposition 5.2.8 satisfies $\xi=\Lambda_{\widehat{\varphi}}(\lambda(g))$ for some non-zero $g \in L^{1}(\mathbb{G})$ such that $\lambda(g) \in \mathcal{N}_{\widehat{\varphi}} \cap \mathcal{D}\left(\widehat{\sigma}_{i / 2}\right)$, where $\widehat{\sigma}$ denotes the modular automorphism group of the dual weight $\widehat{\varphi}$. Then for all $f \in L^{1}(\mathbb{G})$ such that $\lambda(f) \in \mathcal{T}_{\widehat{\varphi}}$, we have

$$
\begin{aligned}
\Lambda_{\widehat{\varphi}}(\lambda(f \star g)) & =\lambda(f) \Lambda_{\widehat{\varphi}}(\lambda(g)) \\
& =\widehat{J} \widehat{\tau}_{-i / 2}\left(\lambda(f)^{*}\right) \widehat{J} \Lambda_{\widehat{\varphi}}(\lambda(g)) \\
& =\widehat{J}_{-i / 2}\left(\lambda(f)^{*}\right) \Lambda_{\widehat{\varphi}}\left(\widehat{\sigma}_{i / 2}(\lambda(g))^{*}\right) \\
& =\widehat{J} \Lambda_{\widehat{\varphi}}\left(\widehat{\tau}_{-i / 2}\left(\lambda(f)^{*}\right) \widehat{\sigma}_{i / 2}(\lambda(g))^{*}\right) \\
& =\Lambda_{\widehat{\varphi}}\left(\widehat{\sigma}_{i / 2}\left(\widehat{\tau}_{-i / 2}\left(\lambda(f)^{*}\right) \widehat{\sigma}_{i / 2}(\lambda(g))^{*}\right)^{*}\right) \\
& =\Lambda_{\widehat{\varphi}}\left(\widehat{\sigma}_{-i / 2}\left(\widehat{\sigma}_{i / 2}(\lambda(g)) \widehat{\tau}_{-i / 2}\left(\lambda(f)^{*}\right)^{*}\right)\right) \\
& =\Lambda_{\widehat{\varphi}}\left(\lambda(g) \widehat{\sigma}_{-i / 2}\left(\widehat{\tau}_{i / 2}(\lambda(f))\right)\right) .
\end{aligned}
$$

Thus, we arrive at the following commutation relation

$$
\lambda(f \star g)=\lambda(g) \widehat{\sigma}_{-i / 2}\left(\widehat{\tau}_{i / 2}(\lambda(f))\right), \quad \lambda(f) \in \mathcal{T}_{\widehat{\varphi}} .
$$

If this is satisfied, then by density of $\left\{f \in L^{1}(\mathbb{G}) \mid \lambda(f) \in \mathcal{T}_{\hat{\varphi}}\right\}$ in $L^{1}(\mathbb{G})[9$, Lemma 4.1], together with the fact that $\widehat{S}^{-1}(\lambda(f))=(f \otimes \mathrm{id})\left(W^{*}\right), f \in L^{1}(\mathbb{G})$, equation (5.8) holds. In summary, we have shown the following. 
Proposition 5.2.9. Let $\mathbb{G}$ be a locally compact quantum group. If there exits a nonzero $g \in L^{1}(\mathbb{G})$ satisfying $\lambda(g) \in \mathcal{N}_{\widehat{\varphi}} \cap \mathcal{D}\left(\widehat{\sigma}_{i / 2}\right)$ and

$$
\lambda(f \star g)=\lambda(g) \widehat{\sigma}_{-i / 2}\left(\widehat{\tau}_{i / 2}(\lambda(f))\right), \quad \lambda(f) \in \mathcal{T}_{\widehat{\varphi}},
$$

then $\mathbb{G}$ is $I N$.

If $\mathbb{G}$ is a Kac algebra, then $\widehat{\tau}_{i / 2}=\mathrm{id}$, and the commutation relation (5.10) reduces to

$$
\lambda(f \star g)=\lambda(g) \widehat{\sigma}_{-i / 2}(\lambda(f)), \quad \lambda(f) \in \mathcal{T}_{\widehat{\varphi}} .
$$

Moreover, if $\mathbb{G}$ is unimodular, meaning $\varphi=\varphi \circ R$, then $\widehat{\sigma}_{i / 2}=$ id [63, Proposition 8.9] and the relation further reduces to

$$
\lambda(f \star g)=\lambda(g) \lambda(f)=\lambda(g \star f), \quad \lambda(f) \in \mathcal{T}_{\widehat{\varphi}} .
$$

Therefore, if $\mathbb{G}$ is a unimodular Kac algebra, then a sufficient condition to be IN is $\mathcal{Z}\left(L^{1}(\mathbb{G})\right) \cap \lambda^{-1}\left(\mathcal{N}_{\hat{\varphi}}\right) \neq\{0\}$. For example, any discrete Kac algebra is IN, as $\lambda^{-1}\left(\mathcal{N}_{\widehat{\varphi}}\right)=L^{1}(\mathbb{G})$ in that case. Recall that Mozak's theorem ensures the equivalence of IN and $\mathcal{Z}\left(L^{1}(\mathbb{G})\right) \neq\{0\}$ in the commutative case $\mathbb{G}=\mathbb{G}_{a}$ [73]. In general, the equivalence of IN and having non-trivial center $\mathcal{Z}\left(L^{1}(\mathbb{G})\right.$ ) remains open (note that one direction is obvious).

Remark 5.2.10. A locally compact quantum group $\mathbb{G}$ is said to be $\operatorname{SIN}$ if $\operatorname{LUC}(\mathbb{G})=$ RUC $(\mathbb{G})[47]$. All compact and discrete quantum groups are SIN, and a commutative quantum group is SIN precisely when its underlying locally compact group is SIN [72]. Contrary to the group case, however, it is not clear whether every SIN quantum group is IN. 


\subsubsection{Relative Injectivity}

In [91, Lemma 4.1] Ruan and Xu showed that the dual $L^{\infty}(\widehat{\mathbb{G}})$ of a strongly inner amenable Kac algebra $\mathbb{G}$ is relatively 1-injective in $L^{1}(\widehat{\mathbb{G}})-\bmod$. We will now show that relative 1-injectivity follows from the a priori weaker notion of inner amenability. In the process we will also show that inner amenability passes to the character group.

Proposition 5.2.11. Let $\mathbb{G}$ be a locally compact quantum group. Consider the following conditions:

1. $\widehat{\mathbb{G}}$ is inner amenable;

2. $L^{\infty}(\mathbb{G})$ is relatively 1 -injective in $L^{1}(\mathbb{G})-\bmod$;

3. $\widetilde{\widehat{G}}=\operatorname{Gr}(\mathbb{G})$ is inner amenable.

Then $(1) \Rightarrow(2) \Rightarrow(3)$. When $\mathbb{G}$ is co-commutative, the conditions are equivalent.

Proof. $(1) \Rightarrow(2)$ : By [64, Proposition 2.15], the unitary operator $U \otimes U:=\widehat{J} J \otimes \widehat{J} J$ on $L^{2}(\mathbb{G}) \otimes L^{2}(\mathbb{G})$ intertwines the right fundamental unitaries of $\widehat{\mathbb{G}}$ and $\widehat{\mathbb{G}}^{\prime}$, denoted $\widehat{V}$ and $\widehat{V}^{\prime}$, respectively. Similarly, $U \otimes U$ intertwines the left fundamental unitaries of $\widehat{\mathbb{G}}$ and $\widehat{\mathbb{G}}^{\prime}$, denoted by $\widehat{W}$ and $\widehat{W}^{\prime}$, respectively. One then obtains a one-to-one correspondence between inner invariant means on $\mathcal{B}\left(L^{2}(\mathbb{G})\right)$ with respect to $\widehat{\mathbb{G}}$ and $\widehat{\mathbb{G}}^{\prime}$ via conjugation with $U$, making $\widehat{\mathbb{G}}$ inner amenable if and only if $\widehat{\mathbb{G}}^{\prime}$ is. Thus, assuming inner amenability of $\widehat{\mathbb{G}}$, we let $\widehat{m}^{\prime} \in \mathcal{B}\left(L^{2}(\mathbb{G})\right)^{*}$ be an inner invariant mean with respect to $\widehat{\mathbb{G}}^{\prime}$.

By Proposition 4.3.3, it suffices to provide a completely contractive morphism which is a left inverse to the map $\Delta: L^{\infty}(\mathbb{G}) \rightarrow \mathcal{C B}\left(L^{1}(\mathbb{G}), L^{\infty}(\mathbb{G})\right)$ given by

$$
\Delta(x)(f)=x \triangleright f, \quad T \in \mathcal{B}\left(L^{2}(\mathbb{G})\right), \rho \in \mathcal{T}\left(L^{2}(\mathbb{G})\right) .
$$

Identifying $\mathcal{C B}\left(L^{1}(\mathbb{G}), L^{\infty}(\mathbb{G})\right) \cong L^{\infty}(\mathbb{G}) \bar{\otimes} L^{\infty}(\mathbb{G})$ via

$$
\langle\Phi, f \otimes g\rangle=\langle\Phi(f), g\rangle, \quad \Phi \in \mathcal{C B}\left(L^{1}(\mathbb{G}), L^{\infty}(\mathbb{G})\right), f, g \in L^{1}(\mathbb{G})
$$


we have $\Delta=\Gamma$, and that the corresponding $L^{1}(\mathbb{G})$-module structure on $L^{\infty}(\mathbb{G}) \bar{\otimes} L^{\infty}(\mathbb{G})$ is defined by $X \triangleright f=(f \otimes \mathrm{id} \otimes \mathrm{id})\left(\Gamma^{r} \otimes \mathrm{id}\right)(X)$ for $X \in L^{\infty}(\mathbb{G}) \bar{\otimes} L^{\infty}(\mathbb{G})$ and $f \in L^{1}(\mathbb{G})$.

We proceed along similar lines as in Theorem 4.3.12. First, consider the map $\Phi: \mathcal{B}\left(L^{2}(\mathbb{G})\right) \bar{\otimes} L^{\infty}(\mathbb{G}) \rightarrow L^{\infty}(\mathbb{G})$ defined by

$$
\Phi(A)=\left(\mathrm{id} \otimes \widehat{m}^{\prime}\right)\left(V^{*} A V\right) \quad A \in \mathcal{B}\left(L^{2}(\mathbb{G})\right) \bar{\otimes} L^{\infty}(\mathbb{G}) .
$$

Clearly, $\Phi$ is a completely contractive left inverse to $\Gamma$. We now show that $\Phi$ is a right $\mathcal{T}_{\triangleright}$-module map. This will complete the proof since Proposition 4.1.6 will entail the invariance $\Phi\left(L^{\infty}(\mathbb{G}) \bar{\otimes} L^{\infty}(\mathbb{G})\right) \subseteq L^{\infty}(\mathbb{G})$, and the restricted module action $\mathcal{T}_{\triangleright} \curvearrowright$ $L^{\infty}(\mathbb{G})$ is the pertinent $L^{1}(\mathbb{G})$-module action. To this end, fix $A \in \mathcal{B}\left(L^{2}(\mathbb{G})\right) \bar{\otimes} L^{\infty}(\mathbb{G})$ and $\rho \in \mathcal{T}\left(L^{2}(\mathbb{G})\right)$. Then

$$
\begin{aligned}
\Phi(A \triangleright \rho) & =\Phi\left((\rho \otimes \mathrm{id} \otimes \mathrm{id})\left(V_{12} A_{13} V_{12}^{*}\right)\right) \\
& =\left(\mathrm{id} \otimes \widehat{m}^{\prime}\right)(\rho \otimes \mathrm{id} \otimes \mathrm{id})\left(V_{23}^{*} V_{12} A_{13} V_{12}^{*} V_{23}\right) \\
& =\left(\mathrm{id} \otimes \widehat{m}^{\prime}\right)(\rho \otimes \mathrm{id} \otimes \mathrm{id})\left(V_{12} V_{23}^{*} V_{13}^{*} A_{13} V_{13} V_{23} V_{12}^{*}\right) \\
& =(\rho \otimes \mathrm{id})\left(V\left(\mathrm{id} \otimes \mathrm{id} \otimes \widehat{m}^{\prime}\right)\left(V_{23}^{*} V_{13}^{*} A_{13} V_{13} V_{23}\right) V^{*}\right) .
\end{aligned}
$$

Now, using the fact that $\widehat{V}^{\prime}=\sigma V^{*} \sigma$, where $\sigma$ is the flip map on $L^{2}(\mathbb{G}) \otimes L^{2}(\mathbb{G})$, for any $\tau, \omega \in \mathcal{T}\left(L^{2}(\mathbb{G})\right)$, we have

$$
\begin{aligned}
& \left\langle\left(\mathrm{id} \otimes \mathrm{id} \otimes \widehat{m}^{\prime}\right)\left(V_{23}^{*} V_{13}^{*} A_{13} V_{13} V_{23}\right), \tau \otimes \omega\right\rangle \\
& =\left\langle\left(\mathrm{id} \otimes \mathrm{id} \otimes \widehat{m}^{\prime}\right)\left(V_{23}^{*}(\sigma \otimes 1) V_{23}^{*} A_{23} V_{23}(\sigma \otimes 1) V_{23}\right), \tau \otimes \omega\right\rangle \\
& =\left\langle\left(\mathrm{id} \otimes \mathrm{id} \otimes \widehat{m}^{\prime}\right)\left(V_{13}^{*} V_{23}^{*} A_{23} V_{23} V_{13}\right), \omega \otimes \tau\right\rangle \\
& =\left\langle\left(\mathrm{id} \otimes \widehat{m}^{\prime}\right)\left(V^{*}\left(1 \otimes(\tau \otimes \mathrm{id})\left(V^{*} A V\right)\right) V\right), \omega\right\rangle \\
& =\left\langle\left(\widehat{m}^{\prime} \otimes \mathrm{id}\right)\left(\widehat{V}^{\prime}\left((\tau \otimes \mathrm{id})\left(V^{*} A V\right) \otimes 1\right) \widehat{V}^{\prime *}\right), \omega\right\rangle \\
& =\left\langle\widehat{m}^{\prime}, \omega \widehat{\triangleright}^{\prime}\left((\tau \otimes \mathrm{id})\left(V^{*} A V\right)\right)\right\rangle \\
& =\left\langle\widehat{m}^{\prime},\left((\tau \otimes \mathrm{id})\left(V^{*} A V\right)\right) \widehat{\triangleleft}^{\prime} \omega\right\rangle .
\end{aligned}
$$


But $(\tau \otimes \mathrm{id})\left(V^{*} A V\right) \in L^{\infty}(\mathbb{G})$ and $\widehat{W}^{\prime} \in L^{\infty}\left(\widehat{\mathbb{G}}^{\prime}\right) \bar{\otimes} L^{\infty}(\mathbb{G})^{\prime}$, so

$$
\begin{aligned}
\left\langle\widehat{m}^{\prime},\left((\tau \otimes \mathrm{id})\left(V^{*} A V\right)\right) \widehat{\triangleleft}^{\prime} \omega\right\rangle & =\left\langle\widehat{m}^{\prime},(\omega \otimes \mathrm{id})\left(\widehat{W}^{\prime *}\left(1 \otimes\left((\tau \otimes \mathrm{id})\left(V^{*} A V\right)\right)\right) \widehat{W}^{\prime}\right)\right\rangle \\
& =\left\langle\widehat{m}^{\prime},(\tau \otimes \mathrm{id})\left(V^{*} A V\right)\right\rangle\langle\omega, 1\rangle \\
& =\left\langle\left(\mathrm{id} \otimes \widehat{m}^{\prime} \otimes \mathrm{id}\right)\left(V^{*} A V \otimes 1\right), \tau \otimes \omega\right\rangle \\
& =\langle\Phi(A) \otimes 1, \tau \otimes \omega\rangle .
\end{aligned}
$$

As $\tau$ and $\omega$ were arbitrary, we have

$$
\begin{aligned}
\Phi(A \triangleright \rho) & =(\rho \otimes \mathrm{id})\left(V\left(\mathrm{id} \otimes \mathrm{id} \otimes \widehat{m}^{\prime}\right)\left(V_{23}^{*} V_{13}^{*} A_{13} V_{13} V_{23}\right) V^{*}\right) \\
& =(\rho \otimes \mathrm{id})\left(V(\Phi(A) \otimes 1) V^{*}\right) \\
& =\Phi(A) \triangleright \rho .
\end{aligned}
$$

$(2) \Rightarrow(3)$ : Recall that $\operatorname{Gr}(\mathbb{G})$ is a group of unitaries in $L^{\infty}(\mathbb{G})$, so it acts naturally on $L^{\infty}(\mathbb{G})$ by conjugation. The existence of a state $m \in L^{\infty}(\mathbb{G})^{*}$ which is $\operatorname{Gr}(\mathbb{G})$ invariant follows directly from the argument of Theorem 5.1.4. Since $\widetilde{\mathbb{G}}$ is a closed quantum subgroup of $\widehat{\mathbb{G}}$ in the sense of Vaes [22, Theorem 5.5], there exists a normal *-homomorphism $\gamma: \widetilde{L^{\infty}(\widetilde{\widehat{\mathbb{G}}})} \rightarrow L^{\infty}(\mathbb{G})$ intertwining the co-multiplications. As $\left.\widetilde{L^{\infty}(\widetilde{\widehat{\mathbb{G}}}}\right)=\mathcal{L}(\widetilde{\widehat{\mathbb{G}}})=\mathcal{L}(\operatorname{Gr}(\mathbb{G}))$, the state $m \circ \gamma \in \mathcal{L}(\operatorname{Gr}(\mathbb{G}))^{*}$ is $\operatorname{Gr}(\mathbb{G})$-invariant, making $\operatorname{Gr}(\mathbb{G})$ inner amenable by Proposition 5.1.2.

When $\mathbb{G}=\mathbb{G}_{s}$ is co-commutative, then $\operatorname{Gr}\left(\mathbb{G}_{s}\right)=G$ and the implication $(3) \Rightarrow(1)$ follows immediately from Proposition 5.1.2.

Remark 5.2.12. In the proof of Proposition 5.2.11, we did not use the full strength of the inner invariant mean $\widehat{m}^{\prime} \in \mathcal{B}\left(L^{2}(\mathbb{G})\right)^{*}$, but rather its restriction to $L^{\infty}(\mathbb{G})$ which was an invariant state for the left action of $L^{1}\left(\widehat{\mathbb{G}}^{\prime}\right)$ on $L^{\infty}(\mathbb{G})$. This weaker notion of inner amenability, which we shall call weak inner amenability of $\widehat{\mathbb{G}}^{\prime}$, has been considered by Kalantar [55]. Inner amenability of $\widehat{\mathbb{G}}^{\prime}$ entails weak inner amenability of $\widehat{\mathbb{G}}^{\prime}$, but their equivalence is not clear beyond the group case. 
Remark 5.2.13. We suspect, but have been unable to prove that relative 1-injectivity of $L^{\infty}(\mathbb{G})$ in $L^{1}(\mathbb{G})-\bmod$ implies inner amenability of $\widehat{\mathbb{G}}$ for general locally compact quantum groups $\mathbb{G}$.

\subsubsection{Biflatness and Biprojectivity}

As in the one-sided case, for a completely contractive Banach algebra $\mathcal{A}$, we say that an operator $\mathcal{A}$-bimodule $X$ is $C$-biflat (respectively, relatively $C$-biflat) if its dual $X^{*}$ is $C$-injective (respectively, relatively $C$-injective) in $\mathcal{A}-\bmod -\mathcal{A}$.

In [91, Theorem 4.3] Ruan and Xu provided a sufficient condition for relative 1-biflatness of $L^{1}(\widehat{\mathbb{G}})$ for any Kac algebra $\mathbb{G}$ by means of the existence of a net of unit vectors $\left(\xi_{\alpha}\right)_{\alpha \in A}$ which are asymptotically invariant under the conjugate corepresentation $W \sigma V \sigma$ and for which $\left.\omega_{\xi_{\alpha}}\right|_{L^{\infty}(\mathbb{G})}$ is a bounded approximate identity of $L^{1}(\mathbb{G})$. In the group setting, this condition is precisely the existence of a quasi-central bounded approximate identity (see $[69,96]$ ). We may now obtain the same conclusion under weaker hypotheses.

Proposition 5.2.14. Let $\mathbb{G}$ be a locally compact quantum group for which there exists a right invariant mean $m \in L^{\infty}(\mathbb{G})^{*}$ satisfying

$$
\left\langle m, \omega \widehat{\triangleright}^{\prime} x\right\rangle=\langle\omega, 1\rangle\langle m, x\rangle, \quad \omega \in \mathcal{T}\left(L^{2}(\mathbb{G})\right), x \in L^{\infty}(\mathbb{G}) .
$$

Then $L^{\infty}(\mathbb{G})$ is relatively 1-injective in $L^{1}(\mathbb{G})-\bmod -L^{1}(\mathbb{G})$. When $\mathbb{G}=\mathbb{G}_{s}$ is co-commutative, the converse is also true.

Proof. By Proposition 4.3.4, it suffices to provide a completely contractive $L^{1}(\mathbb{G})$ bimodule map $\Phi: L^{\infty}(\mathbb{G}) \bar{\otimes} L^{\infty}(\mathbb{G}) \rightarrow L^{\infty}(\mathbb{G})$ which is a left inverse to $\Gamma$. Defining $\Phi(X)=(\mathrm{id} \otimes m)\left(V^{*} X V\right), X \in L^{\infty}(\mathbb{G}) \bar{\otimes} L^{\infty}(\mathbb{G})$, as in Proposition 5.2.11, it immediately follows that $\Phi$ is a completely contractive right $L^{1}(\mathbb{G})$-module map and $\Phi \circ \Gamma=\operatorname{id}_{L^{\infty}(\mathbb{G})}$. However, since $m$ is also a right invariant mean on $L^{\infty}(\mathbb{G})$, the module argument from Theorem 4.3.9 shows that $\Phi$ is also a left $L^{1}(\mathbb{G})$-module map. 
When $\mathbb{G}=\mathbb{G}_{s}$ is co-commutative, then for $f \in L^{1}(G)$ let $\widehat{\Theta}_{f}: \mathcal{L}(G) \bar{\otimes} \mathcal{L}(G) \rightarrow$ $\mathcal{L}(G) \bar{\otimes} \mathcal{L}(G)$ be the normal completely bounded map given by

$$
\widehat{\Theta}_{f}(X)=\int_{G}\left(\lambda(t)^{*} \otimes \lambda(t)^{*}\right) X(\lambda(t) \otimes \lambda(t)) f(t) d t, \quad X \in \mathcal{L}(G) \bar{\otimes} \mathcal{L}(G) .
$$

Then, given a completely contractive $A(G)$-bimodule left inverse $\Phi: \mathcal{L}(G) \bar{\otimes} \mathcal{L}(G) \rightarrow$ $\mathcal{L}(G)$ to $\Gamma_{s}$, it follows as in Theorem 5.1.4 that $\Gamma_{s} \circ \Phi$ is a $\Gamma_{s}(\mathcal{L}(G))$-bimodule map. By Wittstock's bimodule extension theorem [116], this map extends to an $\Gamma_{s}\left(\mathcal{L}(G)\right.$ )-bimodule map $\Psi: \mathcal{B}\left(L^{2}(G)\right) \bar{\otimes} \mathcal{B}\left(L^{2}(G)\right) \rightarrow \mathcal{B}\left(L^{2}(G)\right) \bar{\otimes} \mathcal{B}\left(L^{2}(G)\right)$. Moreover, by [71, Lemma 2.3] we may approximate $\Psi$ in the point weak* topology by a net $\left(\Psi_{\alpha}\right)_{\alpha \in A}$ of normal completely bounded $\Gamma_{s}(\mathcal{L}(G))$-bimodule maps. Thus, for any $X \in \mathcal{L}(G) \bar{\otimes} \mathcal{L}(G)$, we have

$$
\begin{aligned}
\Gamma_{s} \circ \Phi\left(\widehat{\Theta}_{f}(X)\right) & =\Psi\left(\widehat{\Theta}_{f}(X)\right) \\
& =\Psi\left(\int_{G}\left(\lambda(t)^{*} \otimes \lambda(t)^{*}\right) X(\lambda(t) \otimes \lambda(t)) f(t) d t\right) \\
& =w^{*}-\lim _{\alpha} \Psi_{\alpha}\left(\int_{G}\left(\lambda(t)^{*} \otimes \lambda(t)^{*}\right) X(\lambda(t) \otimes \lambda(t)) f(t) d t\right) \\
& =w^{*}-\lim _{\alpha}\left(\int_{G} \Psi_{\alpha}\left(\left(\lambda(t)^{*} \otimes \lambda(t)^{*}\right) X(\lambda(t) \otimes \lambda(t))\right) f(t) d t\right) \\
& =w^{*}-\lim _{\alpha}\left(\int_{G} \Psi_{\alpha}\left(\Gamma_{s}\left(\lambda(t)^{*}\right) X \Gamma_{s}(\lambda(t))\right) f(t) d t\right) \\
& =w^{*}-\lim _{\alpha}\left(\int_{G} \Gamma_{s}\left(\lambda(t)^{*}\right) \Psi_{\alpha}(X) \Gamma_{s}(\lambda(t)) f(t) d t\right) \\
& =w^{*}-\lim _{\alpha} \widehat{\Theta}_{f}\left(\Psi_{\alpha}(X)\right) \\
& =\widehat{\Theta}_{f}(\Psi(X)) \\
& =\widehat{\Theta}_{f}\left(\Gamma_{s} \circ \Phi(X)\right),
\end{aligned}
$$

where we used normality of $\Psi_{\alpha}$ and $\widehat{\Theta}_{f}$ in the fourth and eighth equality, respectively. By definition of $\widehat{\Theta}_{f}$, we have $\widehat{\Theta}_{f} \circ \Gamma_{s}=\Gamma_{s} \circ \widehat{\Theta}^{l}(f)$, so the above calculation entails $\Gamma_{s} \circ \Phi \circ \widehat{\Theta}_{f}=\Gamma_{s} \circ \widehat{\Theta}^{l}(f) \circ \Phi$, which, by injectivity of $\Gamma_{s}$ implies $\Phi \circ \widehat{\Theta}_{f}=\widehat{\Theta}^{l}(f) \circ \Phi$. 
As in the proof of Theorem 5.1.4, the restriction $\left.\Phi\right|_{\mathcal{L}(G) \otimes 1}$ defines a state $m \in$ $\mathcal{L}(G)^{*}$. The bimodule property of $\Phi$ ensures that $m$ is a left invariant mean for the $A(G)$-action on $\mathcal{L}(G)$. Moreover, if $\rho \in \mathcal{T}\left(L^{2}(G)\right)$ with $\left.\rho\right|_{L^{\infty}(G)}=f$, then

$$
\begin{aligned}
\left\langle m, x \triangleleft_{a} \rho\right\rangle & =m\left(\widehat{\Theta}^{l}(f)(x)\right)=m\left(\int_{G} \lambda(s)^{*} x \lambda(s) f(s) d s\right) \\
& =\Phi\left(\int_{G}\left(\lambda(s)^{*} \otimes \lambda(s)^{*}\right)(x \otimes 1)(\lambda(s) \otimes \lambda(s)) f(s) d s\right) \\
& =\Phi\left(\widehat{\Theta}_{f}(x \otimes 1)\right)=\widehat{\Theta}^{l}(f)(\Phi(x \otimes 1)) \\
& =\langle\rho, 1\rangle\langle m, x\rangle
\end{aligned}
$$

for all $x \in \mathcal{L}(G)$. The result then follows from the fact that $V_{s}=W_{a}$ (as shown in $\S 5.2 .1)$. Indeed,

$$
\begin{aligned}
\omega \widehat{\triangleright}_{s}^{\prime} x & =(\mathrm{id} \otimes \omega)\left(\widehat{V}_{s}^{\prime}(x \otimes 1) \widehat{V}_{s}^{\prime *}\right)=(\omega \otimes \mathrm{id})\left(V_{s}^{*}(1 \otimes x) V_{s}\right) \\
& =(\omega \otimes \mathrm{id})\left(W_{a}^{*}(1 \otimes x) W_{a}\right)=x \triangleleft_{a} \omega
\end{aligned}
$$

for all $\omega \in \mathcal{T}\left(L^{2}(G)\right)$ and $x \in \mathcal{L}(G)$. Hence, $m$ also satisfies (5.12).

Remark 5.2.15. In Proposition 5.2.14 we weakened the hypothesis of [91, Theorem 4.3] to the existence of a right invariant mean on $L^{\infty}(\mathbb{G})$ which is invariant under the $\mathcal{T}_{\widehat{D}^{\prime}}$-action of the dual, that is, the weak inner amenability of $\widehat{\mathbb{G}}$ and amenability of $\mathbb{G}$ are realized by the same state. In contrast, Ruan and Xu [91, Theorem 4.3] assume that both the strong inner amenability and co-amenability of $\widehat{\mathbb{G}}$ are realized by the same state.

Remark 5.2.16. Proposition 5.2.14 gives a full characterization of 1-biflatness of the Fourier algebra $A(G)$ in terms of the existence of a state $m \in \mathcal{L}(G)^{*}$ which was invariant under both the right $A(G)$-action and the right $L^{1}(G)$-action on $\mathcal{L}(G)$. We expect that this condition is equivalent to the existence of a quasi-central bounded approximate identity for $L^{1}(G)$, i.e., $G$ is QSIN (see [96]), and are currently working towards a proof in collaboration with Zsolt Tanko. If true, this would provide a partial 
converse to [2, Theorem 2.4], concerning the open question of relative $C$-biflatness of $A(G)$ and its relation to QSIN.

A completely contractive Banach algebra $\mathcal{A}$ is operator amenable if it is relatively $C$-biflat for some $C>0$ and has a bounded approximate identity. This is not the original definition of operator amenability, but was shown to be equivalent in [91, Theorem 2.4]. This notion is the operator module analogue of the classical concept introduced by Johnson [52], who showed that the group algebra $L^{1}(G)$ of a locally compact group $G$ is (operator) amenable if and only if $G$ is amenable. In the breakthrough paper [89], Ruan established the dual result, showing that the Fourier algebra $A(G)$ of a locally compact group $G$ is operator amenable precisely when $G$ is amenable. Since $L^{\infty}(G)$ is a 1-injective operator space, and the $\mathcal{T}_{\widehat{\triangleright}_{a}^{\prime}}$-action is trivial on $L^{\infty}(G)$, it follows from Propositions 4.3.8 and 5.2.14 together with [52] that a locally compact group $G$ is amenable if and only if $L^{1}(G)$ is 1-biflat. Dually, if $G$ were amenable, then $\mathcal{L}(G)$ is an injective operator space, and it is relatively 1-injective in $A(G)-\bmod -A(G)$ by [91, Theorem 4.4]. Thus, Proposition 4.3 .8 together with [89, Theorem 3.6] show that a locally compact group $G$ is amenable if and only if $A(G)$ is 1-biflat. We may therefore interpret the above results as the equivalence

$$
L^{1}(G) \text { is 1-biflat } \Leftrightarrow A(G) \text { is 1-biflat. }
$$

As we will now prove, this is true in much greater generality.

Theorem 5.2.17. Let $\mathbb{G}$ be a locally compact quantum group. Then $L^{1}(\mathbb{G})$ is 1-biflat if and only if $L^{1}(\widehat{\mathbb{G}})$ is 1-biflat.

Proof. Clearly, it suffices to show one direction by quantum Pontrjagin duality, so suppose that $L^{1}(\widehat{\mathbb{G}})$ is 1 -biflat, that is, $L^{\infty}(\widehat{\mathbb{G}})$ is 1 -injective in $L^{1}(\widehat{\mathbb{G}})-\bmod -L^{1}(\widehat{\mathbb{G}})$. Consider the $L^{1}(\widehat{\mathbb{G}})$-bimodule structure on $\mathcal{B}\left(L^{2}(\mathbb{G})\right)$ given by

$$
\hat{f} \widehat{\triangleright} T=(\mathrm{id} \otimes \hat{f}) \widehat{V}(T \otimes 1) \widehat{V}^{*} \quad \text { and } \quad T \widehat{\triangleleft} \hat{f}=(\hat{f} \otimes \mathrm{id}) \widehat{W}^{*}(1 \otimes T) \widehat{W},
$$


for $\hat{f} \in L^{1}(\widehat{\mathbb{G}})$ and $T \in \mathcal{B}\left(L^{2}(\mathbb{G})\right)$. Then by 1-injectivity, $\operatorname{id}_{L^{\infty}(\widehat{\mathbb{G}})}$ extends to a completely contractive $L^{1}(\widehat{\mathbb{G}})$-bimodule projection $E: \mathcal{B}\left(L^{2}(\mathbb{G})\right) \rightarrow L^{\infty}(\widehat{\mathbb{G}})$. By the left $\mathcal{T}_{\bar{D}}$-module property, it follows from the standard argument that $E\left(L^{\infty}(\mathbb{G})\right) \subseteq$ $L^{\infty}(\mathbb{G}) \cap L^{\infty}(\widehat{\mathbb{G}})=\mathbb{C} 1$. Also, Proposition 4.2 .10 implies that $E$ is a right $\mathcal{T}_{\triangleleft}$-module map. Let $R$ be the extended unitary antipode of $\mathbb{G}$. Then

$$
(R \otimes R)\left(\widehat{V}^{\prime}\right)=(R \otimes R)\left(\sigma V^{*} \sigma\right)=\Sigma(R \otimes R)\left(V^{*}\right)=\Sigma(\widehat{J} \otimes \widehat{J})(V)(\widehat{J} \otimes \widehat{J})=\Sigma \widehat{W},
$$

where the last equality follows from equation (3.5) and the adjoint relations of $W$ and $V$. Let $E_{R}: \mathcal{B}\left(L^{2}(\mathbb{G})\right) \rightarrow L^{\infty}\left(\widehat{\mathbb{G}}^{\prime}\right)$ be the projection of norm one $R \circ E \circ R$. Then for $\omega \in \mathcal{T}\left(L^{2}(\mathbb{G})\right)$ and $T \in \mathcal{B}\left(L^{2}(\mathbb{G})\right)$, we have

$$
\begin{aligned}
E_{R}\left(\omega \widehat{\triangleright}^{\prime} T\right) & =R\left(E\left(R\left((\mathrm{id} \otimes \omega) \widehat{V}^{\prime}(T \otimes 1) \widehat{V}^{*}\right)\right)\right) \\
& =R\left(E\left((\operatorname{id} \otimes \omega \circ R)(R \otimes R)\left(\widehat{V}^{\prime}(T \otimes 1) \widehat{V}^{\prime *}\right)\right)\right) \\
& =R\left(E\left((\operatorname{id} \otimes \omega \circ R)\left((R \otimes R)\left(\widehat{V}^{\prime *}\right)(R(T) \otimes 1)(R \otimes R)\left(\widehat{V}^{\prime}\right)\right)\right)\right) \\
& =R\left(E\left((\operatorname{id} \otimes \omega \circ R)\left(\left(\Sigma \widehat{W}^{*}\right)(R(T) \otimes 1)(\Sigma \widehat{W})\right)\right)\right) \\
& =R\left(E\left((\omega \circ R \otimes \mathrm{id})\left(\widehat{W}^{*}(1 \otimes R(T)) \widehat{W}\right)\right)\right) \\
& =R(E(R(T) \widehat{\triangleleft}(\omega \circ R))) \\
& =R(E(R(T)) \widehat{\triangleleft}(\omega \circ R)) \\
& =\omega \widehat{\triangleright}^{\prime} E_{R}(T) .
\end{aligned}
$$

Thus, $E_{R}$ is a left $\mathcal{T}_{\widehat{\triangleright}^{\prime}}$-module map. Since $R\left(L^{\infty}(\mathbb{G})\right)=L^{\infty}(\mathbb{G})$, the restriction $\left.E_{R}\right|_{L^{\infty}(\mathbb{G})}$ defines a state $m \in L^{\infty}(\mathbb{G})^{*}$ satisfying

$$
\left\langle m, \omega \widehat{\triangleright}^{\prime} x\right\rangle=\langle\omega, 1\rangle\langle m, x\rangle, \quad \omega \in \mathcal{T}\left(L^{2}(\mathbb{G})\right), x \in L^{\infty}(\mathbb{G})
$$

But $E$ was also a right $\mathcal{T}_{\triangleleft}$-module map, which implies that $E_{R}$ is a left $\mathcal{T}_{\triangleright}$-module 
map by the generalized antipode relation (4.3). Thus, we also have

$$
\langle m, \omega \triangleright x\rangle=\langle\omega, 1\rangle\langle m, x\rangle, \quad \omega \in \mathcal{T}\left(L^{2}(\mathbb{G})\right), x \in L^{\infty}(\mathbb{G}),
$$

meaning that $m$ is a right invariant mean on $L^{\infty}(\mathbb{G})$. By Proposition 5.2.14 it follows that $L^{\infty}(\mathbb{G})$ is relatively 1-injective in $L^{1}(\mathbb{G})-\bmod -L^{1}(\mathbb{G})$.

By 1-injectivity of $L^{\infty}(\widehat{\mathbb{G}})$ in $L^{1}(\widehat{\mathbb{G}})-\bmod -L^{1}(\widehat{\mathbb{G}})$, there exists a completely contractive morphism $\Phi: L^{\infty}(\widehat{\mathbb{G}}) \bar{\otimes} L^{\infty}(\widehat{\mathbb{G}}) \rightarrow L^{\infty}(\widehat{\mathbb{G}})$ which is a left inverse to $\widehat{\Gamma}$. It follows that $\left.\Phi\right|_{L^{\infty}(\widehat{\mathbb{G}}) \otimes 1}$ defines a state $\widehat{m} \in L^{\infty}(\widehat{\mathbb{G}})^{*}$ which is a right $L^{1}(\widehat{\mathbb{G}})$-module map, i.e., $\widehat{\mathbb{G}}$ is amenable. Hence, $L^{\infty}(\mathbb{G})$ is a 1-injective operator space by Theorem 4.2.2. Proposition 4.3 .8 then entails the 1 -injectivity of $L^{\infty}(\mathbb{G})$ in $L^{1}(\mathbb{G})-\bmod -$ $L^{1}(\mathbb{G})$.

The relative biprojectivity of $L^{1}(\mathbb{G})$, that is, relative projectivity of $L^{1}(\mathbb{G})$ as an operator bimodule over itself, has been completely characterized: $L^{1}(\mathbb{G})$ is relatively $C$-biprojective if and only if $L^{1}(\mathbb{G})$ is relatively 1-biprojective if and only if $\mathbb{G}$ is a compact Kac algebra $[1,19,11]$. The corresponding characterization for (relative) $C$ biflatness is wide open. Remarkably, if a compact quantum group $\mathbb{G}$ has a relatively $C$-biflat convolution algebra $L^{1}(\mathbb{G})$, then it must be a Kac algebra [11, Theorem 1.1].

We finish this section with a generalization of [56, Theorem 4.9] beyond coamenable quantum groups, which at the same time characterizes the 1-biprojectivity of $L^{1}(\mathbb{G})$.

Theorem 5.2.18. Let $\mathbb{G}$ be a locally compact quantum group. Then the following conditions are equivalent:

1. $\mathbb{G}$ is finite-dimensional.

2. $\mathcal{T}_{\triangleright}$ is relatively 1-biprojective;

3. $L^{1}(\mathbb{G})$ is 1-biprojective; 
Proof. (1) $\Rightarrow$ (2) follows from [56, Theorem 4.9].

(2) $\Rightarrow$ (3) follows similarly to the proof of Theorem 4.4.13, giving the relative 1-biprojectivity of $L^{1}(\mathbb{G})$ together with the 1-biprojectivity of $L^{1}(\mathbb{G})$ as an operator space. Proposition 4.3.7 then yields (3).

(3) $\Rightarrow(1)$ The 1-biprojectivity of $L^{1}(\mathbb{G})$ ensures the existence of a normal completely bounded $L^{1}(\mathbb{G})$-bimodule left inverse $\Phi: L^{\infty}(\mathbb{G}) \bar{\otimes} L^{\infty}(\mathbb{G}) \rightarrow L^{\infty}(\mathbb{G})$ to $\Gamma$. As usual, the restriction $\left.\Phi\right|_{L^{\infty}(\mathbb{G}) \otimes 1}: L^{\infty}(\mathbb{G}) \rightarrow L^{\infty}(\mathbb{G})$ maps into $\mathbb{C}$, and, moreover, it is a right $L^{1}(\mathbb{G})$-module map, so $\mathbb{G}$ is compact by normality of $\Phi$. Since compact quantum groups are regular, we may repeat the proof of $(3) \Rightarrow(1)$ from Theorem 4.4.13 to deduce the discreteness of $\mathbb{G}$. Thus, $\mathbb{G}$ is finite-dimensional by [56, Theorem $4.8]$.

Remark 5.2.19. As in Remark 4.4.15, it is not clear whether we can replace relative 1-biprojectivity of $\mathcal{T}_{\triangleright}$ with 1-biprojectivity of $\mathcal{T}_{\triangleright}$ in Theorem 5.2.18. 


\section{Chapter 6}

\section{Future Directions}

We believe there are many interesting avenues of research that are generated by this thesis. The first, is to study the non-relative homological properties of various $L^{1}(\mathbb{G})$-modules of interest in abstract harmonic analysis, such as $C_{0}(\mathbb{G}), M(\mathbb{G})$ and $L^{2}(\mathbb{G})$. Another, would be a complete systematic investigation of the concept of inner amenability for locally compact quantum groups. We have clearly only scratched the surface, and further analysis should lead to interesting examples. In particular, to what extent can the well-known results surrounding IN and SIN groups be generalized to locally compact quantum groups?

Given a locally compact quantum group $\mathbb{G}$ and a von Neumann algebra $M$, a left co-action of $\mathbb{G}$ on $M$ is a normal, unital, injective $*$-homomorphism $\alpha: M \rightarrow$ $L^{\infty}(\mathbb{G}) \bar{\otimes} M$ satisfying (id $\left.\otimes \alpha\right) \circ \alpha=(\Gamma \otimes$ id) $\circ \alpha$. Taking a faithful representation $M \subseteq \mathcal{B}(H)$, the crossed product of $M$ by $\mathbb{G}$, denoted $M \rtimes_{\alpha} \mathbb{G}$ is defined as the von Neumann subalgebra of $\mathcal{B}\left(L^{2}(\mathbb{G})\right) \bar{\otimes} \mathcal{B}(H)$ generated by $\alpha(M)$ and $L^{\infty}(\widehat{\mathbb{G}}) \otimes 1$. It is

well-known that there exits an co-action of the dual $L^{\infty}(\widehat{\mathbb{G}})$, and hence an operator $L^{1}(\widehat{\mathbb{G}})$-module structure on the crossed product $M \rtimes_{\alpha} \mathbb{G}$ (see [107]). As in the group case, there is a notion of Zimmer amenability for co-actions of quantum groups, so a natural question is to seek a characterization of Zimmer amenability in terms of injectivity of the crossed product under the induced $L^{1}(\widehat{\mathbb{G}})$-module structure. Indeed, 
when $M=\mathbb{C} 1$ and $\alpha$ is trivial, we have $M \rtimes_{\alpha} \mathbb{G}=L^{\infty}(\widehat{\mathbb{G}})$, and the action is Zimmer amenable precisely when $\mathbb{G}$ is amenable, that is, when $L^{\infty}(\widehat{\mathbb{G}})$ is 1 -injective as an operator $L^{1}(\widehat{\mathbb{G}})$-module.

Finally, having refined injectivity of group von Neumann algebras $\mathcal{L}(G)$ through a covariance condition, that is, injectivity as an operator $A(G)$-module, a natural question is to seek a similar manifestation at the level of group $C^{*}$-algebras. In other words, is there a notion of "covariant nuclearity" which captures amenability of the underlying group? One may ask similar questions regarding weaker approximation properties such has weak amenability and the Haagerup property. One is then led to consider an analogue of the Grothendieck programme for Banach modules, including module approximation properties and mapping spaces of morphisms. Similar ideas have appeared previously for modules over $C^{*}$-algebras, in particular, Hilbert $C^{*}$ modules, however there has been relatively little concerning operator modules over more general completely contractive Banach algebras. With the theory of quantum groups currently under heavy investigation, and whose underlying structure is governed by operator modules over non- $C^{*}$-algebras, it seems natural to pursue these questions in the near future. 


\section{Bibliography}

[1] ARISTOV, O. Y., Amenability and compact type for Hopf-von Neumann algebras from the homological point of view. Banach algebras and their applications, 15-37, Contemp. Math., 363, Amer. Math. Soc., Providence, RI, 2004.

[2] ARISTOV, O. Y., RUNDE, V., SPRONK, N., Operator biflatness of the Fourier algebra and approximate indicators for subgroups. J. Funct. Anal. 209 (2004), no. 2, 367-387.

[3] ARVESON, W. B., Subalgebras of $C^{*}$-algebras. Acta Math. 123 (1969), 141-224.

[4] BAAJ, S., SKANDALIS, G., Unitaires multiplicatifs et dualité pour les produits croisé de $C^{*}$-algèbres. Ann. Sci. École Norm. Sup. 26 (1993), no. 4, 425-488.

[5] BANICA, T., Le groupe quantique compact libre $U(n)$. Comm. Math. Phys. 190 (1997), 143-172.

[6] BÉDOS, E., TUSET, L., Amenability and co-amenability for locally compact quantum groups. Internat. J. Math. 14 (2003), no. 8, 865-884.

[7] BEKKA, M. E., Amenable unitary representations of locally compact groups. Invent. Math. 100 (1990), no. 2, 383-401.

[8] BLECHER, D. P., The standard dual of an operator space. Pacific J. Math. 153 (1992), no. 1, 15-30.

[9] BRANNAN, M., DAWS, M., SAMEI, E., Completely bounded representations of convolution algebras of locally compact quantum groups. Münster J. Math. 6 (2013), 445-482.

[10] DE CANNIÈRE, J., HAAGERUP, U., Multipliers of the Fourier algebras of some simple Lie groups and their discrete subgroups. Amer. J. Math. 107 (1985), no. 2, 455-500.

[11] CASPERS, M., LEE, H. H., RICARD, E., Operator biflatness of the $L^{1}$-algebras of compact quantum groups. J. Reine Angew. Math. 700 (2015), 235-244. 
[12] CHOI, M.-D., A Schwarz inequality for positive linear maps on $C^{*}$-algebras. Illinois J. Math. 18 (1974), 565-574.

[13] CHOI, M.-D., EFFROS, E. G., Nuclear $C^{*}$-algebras and injectivity: the general case. Indiana Univ. Math. J. 26 (1977), no. 3, 443-446.

[14] CHRISTENSEN, E., SINCLAIR, A. M., On von Neumann algebras which are complemented subspaces of $\mathcal{B}(H)$. J. Funct. Anal. 122 (1994), no. 1, 91-102.

[15] CONNES, A., Classification of injective factors. Cases $I I_{1}, I I_{\infty}, I I I_{\lambda}, \lambda \neq 1$. Ann. of Math. (2) 104 (1976), no. 1, 73-115.

[16] COWLING, M., HAAGERUP, U., Completely bounded multipliers of the Fourier algebra of a simple Lie group of real rank one. Invent. Math. 96 (1989), no. 3, 507-549.

[17] CRANN, J., NEUFANG, M., Amenability and covariant injectivity of locally compact quantum groups. Trans. Amer. Math. Soc. To appear. ArXiv:1208.2986.

[18] DALES, H. G., POLYAKOV, M. E., Homological properties of modules over group algebras. Proc. London Math. Soc. (3) 89 (2004), no. 2, 390-426.

[19] DAWS, M., Operator biprojectivity of compact quantum groups. Proc. Amer. Math. Soc. 138 (2010), no. 4, 1349-1359.

[20] DAWS, M., Multipliers, self-induced and dual Banach algebras. Dissertationes Math. 470 (2010).

[21] DAWS, M., Completely positive multipliers of quantum groups. Internat. J. Math. 23 (2012), no. 12, 1250132.

[22] DAWS, M., Categorical aspects of quantum groups: multipliers and intrinsic groups, Canad. J. Math. To appear. ArXiv:1410.2156.

[23] DAWS, M., KASPRZAK, P., SKALSKI, A., SOŁTAN, P. M., Closed quantum subgroups of locally compact quantum groups. Adv. Math. 231 (2012), no. 6, 3473-3501.

[24] DE CANNIÈRE, J., HAAGERUP, U., Multipliers of the Fourier algebras of some simple Lie groups and their discrete subgroups. Amer. J. Math. 107 (1985), no. 2, 455-500.

[25] DE LA HARPE, P., Moyennabilité du groupe unitaire et propriété P de Schwartz des algèbres de von Neumann. Algèbres d'opérateurs (Sém., Les Plans-sur-Bex, 1978), pp. 220-227, Lecture Notes in Math., 725, Springer, Berlin, 1979.

[26] DESMEDT, P., QUAEGEBEUR, J., VAES, S., Amenability and the bicrossed product construction. Illinois J. Math. 46 (2002), no. 4, 1259-1277. 
[27] Effros, E. G., Property $\Gamma$ and inner amenability. Proc. Amer. Math. Soc. 47 (1975), 483-486.

[28] EFFROS, E. G., LANCE, C. E., Tensor products of operator algebras. Adv. Math. 25 (1977), no. 1, 1-34.

[29] EFFROS, E. G., RUAN, Z.-J., Operator Spaces. London Mathematical Society Monographs, New Series 23, Oxford University Press, New York, 2000.

[30] ENOCK, M., SCHWARTZ, J. M., Kac Algebras and Duality of Locally Compact Groups. Springer-Verlag, Berlin, 1992.

[31] EYMARD, P., L'algèbre de Fourier d'un groupe localement compact. Bull. Soc. Math. France 92 (1964), 181-236.

[32] FOLlAnd, G. B., A Course in Abstract Harmonic Analysis. Studies in Advanced Mathematics, CRC Press Incorporated, Florida, 1995.

[33] FORREST, B. E., LEE, H. H., SAMEI, E., Projectivity of modules over Fourier algebras. Proc. Lond. Math. Soc. (3) 102 (2011), no. 4, 697-730.

[34] GHAHRAMANI, F., Isometric representations of $M(G)$ on $\mathcal{B}(H)$. Glasgow Math. J. 23 (1982), no. 2, 119-122.

[35] GANEI, M. R., NASR-ISFAHANI, R., Inner amenability of locally compact quantum groups. Internat. J. Math. 24 (2013), no. 7, 1350058.

[36] GIORDANO, T., PESTOV, V., Some extremely amenable groups related to operator algebras and ergodic theory. J. Inst. Math. Jussieu 6 (2007), no. 2, 279-315.

[37] GRANIRER, E., Weakly almost periodic and uniformly continuous functionals on the Fourier algebra of any locally compact group. Trans. Amer. Math. Soc. 189 (1974), 371-382.

[38] GRANIRER, E., LAU. A. T.-M., Invariant means on locally compact groups. Illinois J. Math. 15 (1971), 249-257.

[39] GUICHARDET, A., Tensor products of $C^{*}$-algebras. Aarhus University Lecture Notes Series no. 12, 1969.

[40] HAAGERUP, U., The standard from of von Neumann algebras. Math. Scand. 37 (1975), 271-283.

[41] HAAGERUP, U.,, Decomposition of completely bounded maps on operator algebras. Unpublished, Odense University, Denmark, 1980. 
[42] HAAGERUP, U., Injectivity and decomposition of completely bounded maps. Operator algebras and their connections with topology and ergodic theory (Busteni, 1983), 170-222, Lecture Notes in Math., 1132, Springer, Berlin, 1985.

[43] HAAGERUP, U., KRAUS, J., Approximation properties for group $C^{*}$-algebras and group von Neumann algebras. Trans. Amer. Math. Soc. 344 (1994), no.2, 667-699.

[44] HELEMSKII, A. Y., Metric version of flatness and Hahn-Banach type theorems for normed modules over sequence algebras. Studia Math. 206 (2011), no. 2, 135160.

[45] HELEMSKII, A. Y., Banach and Locally Convex Algebras. Translated from the Russian by A. West. Oxford Science Publications. The Clarendon Press, Oxford University Press, New York, 1993.

[46] HERZ, C., Harmonic synthesis for subgroups. Ann. Inst. Fourier (Grenoble) 23 (1973), no. 3, 91-123.

[47] HU, Z., NEUFANG, M., RUAN, Z.-J., On topological centre problems and SIN quantum groups. J. Funct. Anal. 257 (2009), no. 2, 610-640.

[48] HU, Z., NEUFANG, M., RUAN, Z.-J., Multipliers on a new class of Banach algebras, locally compact quantum groups, and topological centres. Proc. Lond. Math. Soc. (3) 100 (2010), no. 2, 429-458.

[49] HU, Z., NEUFANG, M., RUAN, Z.-J., Completely bounded multipliers over locally compact quantum groups. Proc. Lond. Math. Soc. (3) 103 (2011), no. 1, $1-39$.

[50] HU, Z., NEUfANG, M., RUAN, Z.-J., Module maps over locally compact quantum groups. Studia Math. 211 (2012), no. 2, 111-145.

[51] HU, Z., NEUFANG, M., RUAN, Z.-J., Convolution of trace class operators over locally compact quantum groups. Canad. J. Math. 65 (2013), no. 5, 1043-1072.

[52] JOHNSON, B. E., Cohomology in Banach algebras. Memoirs of the American Mathematical Society, No. 127. American Mathematical Society, Providence, R.I., 1972.

[53] JUNGE, M., NEUFANG, M., RUAN, Z.-J., A representation theorem for locally compact quantum groups. Internat. J. Math. 20 (2009), no. 3, 377-400.

[54] KADISON, R. V., RINGROSE, J. R., Fundamentals of the Theory of Operator Algebras, Volume II: Advanced Theory. Graduate Studies in Mathematics, 16. American Mathematical Society, Providence, RI, 1997. 
[55] KALANTAR, M., Private communication.

[56] KALANTAR, M., NEUFANG, M., Duality, cohomology, and geometry of locally compact quantum groups. J. Math. Anal. Appl. 406 (2013), no. 1, 22-33.

[57] KALANTAR, M., NEUFANG, M., From quantum groups to groups. Canad. J. Math. 65 (2013), no. 5, 1073-1094.

[58] KASPRZAK, P., SOŁTAN, P. M., Embeddable quantum homogeneous spaces. J. Math. Anal. Appl. 411 (2014), no. 2, 574-591.

[59] KRAUS, J., RUAN, Z.-J., Multipliers of Kac algebras. Internat. J. Math. 8 (1997), no. 2, 213-248.

[60] KRAUS, J., RUAN, Z.-J., Approximation properties for Kac algebras. Indiana Univ. Math. J. 48 (1999), no. 2, 469-535.

[61] KUSTERMANS, J., Locally compact quantum groups in the universal setting. Internat. J. Math. 12 (2001), no. 3, 289-338.

[62] KUSTERMANS, J., One-parameter representations on $C^{*}$-algebras. Preprint Odense Universitet, 1997.

[63] KUSTERMANS, J., VAES, S., Locally compact quantum groups. Ann. Sci. École Norm. Sup. (4) 33 (2000), no. 6, 837-934.

[64] KUSTERMANS, J., VAES, S., Locally compact quantum groups in the von Neumann algebraic setting. Math. Scand. 92 (2003), no. 1, 68-92.

[65] LANCE, C., On nuclear $C^{*}$-algebras. J. Funct. Anal. 12 (1973), 157-176.

[66] LAU, A. T.-M., PATERSON, A. L. T., Inner amenable locally compact groups. Trans. Amer. Math. Soc. 325 (1991), no. 1, 155-169.

[67] LAU, A. T.-M., PATERSON, A. L. T., Group amenability properties for von Neumann algebras. Indiana Univ. Math. J. 55 (2006), no. 4, 1363-1388.

[68] LEPTIN, H., Sur l'algèbre de Fourier d'un groupe localement compact. C. R. Acad. Sci. Paris Sér. A-B 266 (1968), A1180-A1182.

[69] LOSERT, V., RINDLER, H., Asymptotically central functions and invariant extensions of Dirac measure. Probability measures on groups, VII (Oberwolfach, 1983), 368-378, Lecture Notes in Math., 1064, Springer, Berlin, 1984.

[70] MAJID, S., Hopf-von Neumann algebra bicrossproducts, Kac algebra bicrossproducts, and the classical Yang-Baxter equations. J. Funct. Anal. 95 (1991), no. 2, 291-319. 
[71] MAY, G., NEUHARDT, E., WITTSTOCK, G., The space of completely bounded module homomorphisms. Arch. Math. (Basel) 53 (1989), no. 3, 283-287.

[72] MILNES, P., Uniformity and uniformly continuous functions for locally compact groups. Proc. Amer. Math. Soc. 109 (1990), no. 2, 567-570.

[73] MOSAK, R. D., Central functions in group algebras. Proc. Amer. Math. Soc. 29 (1971), 613-616.

[74] NEUFANG, M., Abstrakte Harmonische Analyse und Modulhomomorphismen ü von Neumann-Algebren. Ph.D. Thesis at University of Saarland, Saarbrücken, Germany, 2000.

[75] NEUFANG, M., Amplification of completely bounded operators and Tomiyama's slice maps. J. Funct. Anal. 207 (2004), no. 2, 300-329.

[76] NEUFAnG, M., RUAN, Z.-J., SPRONK, N., Completely isometric representations of $M_{c b} A(G)$ and $U C B(\widehat{G})^{*}$. Trans. Amer. Math. Soc. 360 (2008), no. 3, 1133-1161.

[77] NEUFANG, M., RUNDE, V., Harmonic operators: the dual perspective. Math. Z. 255 (2007), no. 3, 669-690.

[78] OKAYASU, R., OZAWA, N., TOMATSU, R., Haagerup approximation property via bimodules. ArXiv:1501.06293.

[79] PAULSEN, V. I., Every completely polynomially bounded operator is similar to a contraction. J. Funct. Anal. 55 (1984), no. 1, 1-17.

[80] PAUlSEN, V. I., Completely Bounded Maps and Operator Algebras. Cambridge University Press, 2002.

[81] PATERSON, A. L. T., Nuclear $C^{*}$-algebras have amenable unitary groups. Proc. Amer. Math. Soc. 114 (1992), no. 3, 719-721.

[82] PATERSON, A. L. T., Amenability. Mathematical Surveys and Monographs 29, American Mathematical Society, Providence, Rhode Island, 1988.

[83] PIRKOVSKII, A. Y., Biprojectivity and biflatness for convolution algebras of nuclear operators. Canad. Math. Bull. 47 (2004), no. 3, 445-455.

[84] PISIER, G., The operator Hilbert space $O H$, complex interpolation and tensor norms. Mem. Amer. Math. Soc. 122 (1996), no. 585.

[85] RENAUD, P. F., Invariant means on a class of von Neumann algebras. Trans. Amer. Math. Soc. 170 (1972), 285-291. 
[86] REN, L.-B., Introduction to Operator Algebras. World Scientific Publishing Co. Pte. Ltd. (1992).

[87] RUAN, Z.-J., Injectivity of operator spaces. Trans. Amer. Math. Soc. 315 (1989), no. $1,89-104$.

[88] RUAN, Z.-J., On the predual of dual algebras. J. Operator Theory 27 (1992), no. 1, 179-192.

[89] RUAN, Z.-J., Operator amenability of $A(G)$. Amer. J. Math. 117 (1995), no. 6, 1449-1474.

[90] RUAN, Z.-J., Amenability of Hopf von Neumann algebras and Kac algebras. J. Funct. Anal. 139 (1996), no. 2, 466-499.

[91] RUAN, Z.-J., XU, G., Splitting properties of operator bimodules and operator amenability of Kac algebras. Operator theory, operator algebras and related topics (Timisoara, 1996), 193-216, Theta Found., Bucharest, 1997.

[92] RUNDE, V., Lectures on Amenability. Lecture Notes in Mathematics 1774, Springer-Verlag Berlin-Heidelberg, 2002.

[93] RUNDE, V., Uniform continuity over locally compact quantum groups. J. Lond. Math. Soc. (2) 80 (2009), no. 1, 55-71.

[94] SALMI, P., Compact quantum subgroups and left invariant $C^{*}$-subalgebras of locally compact quantum groups. J. Funct. Anal. 261 (2011), no. 1, 1-24.

[95] SOŁTAN, P., VISELTER, A., A note on amenability of locally compact quantum groups. Canad. Math. Bull. 57 (2014), no. 2, 424-430.

[96] STOKKE, R., Quasi-central bounded approximate identities in group algebras of locally compact groups. Illinois J. Math. 48 (2004), no. 1, 151-170.

[97] STOKKE, R., Amenable representations and coefficient subspaces of FourierStieltjes algebras. Math. Scand. 98 (2006), no. 2, 182-200.

[98] STØRMER, E., Regular abelian Banach algebras of linear maps of operator algebras. J. Funct. Anal. 37 (1980), 331-373.

[99] TAKESAKI, M., A characterization of group algebras as a converse of TannakaStinespring-Tatsuuma duality theorem. Amer. J. Math. 91 (1969), 529-564.

[100] TAKESAKI, M., Theory of Operator Algebras I. Encyclopedia of Mathematical Sciences 124, Springer-Verlag Berlin-Heidelberg-New York, 2002.

[101] TAKESAKI, M., Theory of Operator Algebras II. Encyclopedia of Mathematical Sciences 125, Springer-Verlag Berlin-Heidelberg-New York, 2003. 
[102] TAKESAKI, M., Theory of Operator Algebras III. Encyclopedia of Mathematical Sciences 127, Springer-Verlag Berlin-Heidelberg-New York, 2003.

[103] TAYLOR, K. F., The type structure of the regular representation of a locally compact group. Math. Ann. 222 (1976), no. 3, 211-224.

[104] TOMATSU, R., Amenable discrete quantum groups. J. Math. Soc. Japan 58 (2006), no. 4, 949-964.

[105] TOMIYAMA, J., On the projection of norm one in $W^{*}$-algebras. Proc. Japan Acad. 33 (1957), 608-612.

[106] VAES, S., Locally Compact Quantum Groups. Ph. D. thesis, K.U. Leuven, 2000.

[107] VAES, S., The unitary implementation of a locally compact quantum group action. J. Funct. Anal. 180 (2001), no. 2, 426-480.

[108] VAES, S., A new approach to quantum and imprimitivity results. J. Funct. Anal. 229 (2005), 317-374.

[109] VAES, S., VAINERMAN, L., On low-dimensional locally compact quantum groups. Locally compact quantum groups and groupoids (Strasbourg, 2002), 127187, IRMA Lect. Math. Theor. Phys., 2, de Gruyter, Berlin, 2003.

[110] VAES, S., VAN DAELE, A., The Heisenberg commutation relations, commuting squares and the Haar measure on locally compact quantum groups. Operator Algebras and Mathematical Physics 379-400 (Constanta, 2001) (Theta, Bucharest, 2003).

[111] VAN DAELE, A., WANG, S., Universal quantum groups. Internat. J. Math. 7, (1996), no. 2, 289-338.

[112] VAN DAELE, A., Locally compact quantum groups. A von Neumann algebra approach. SIGMA 10 (2014), 082, 41 pp.

[113] WENDEL, J. G., Left centralizers and isomorphisms of group algebras. Pacific J. Math 2, 251-261 (1952).

[114] WHITE, M. C., Injective modules for uniform algebras. Proc. London Math. Soc. (3) 73 (1996), no. 1, 155-184.

[115] WITTSTOCK, G., Ein operatorvertiger Hahn-Banach Satz. J. Func. Anal., 40 (1981), 127-50.

[116] WITTSTOCK, G., Extension of completely bounded $C^{*}$-module homomorphisms. Operator algebras and group representations, Vol. II (Neptun, 1980), 238-250, Monogr. Stud. Math., 18, Pitman, Boston, MA, 1984. 
[117] WORONOWICZ, S. L., Twisted SU(2) group. An example of a noncommutative differential calculus. Publ. Res. Inst. Math. Sci. 23 (1987), no. 1, 117-181.

[118] WORONOWICZ, S. L., Compact quantum groups. Symétries quantiques (Les Houches, 1995), 845-884, North-Holland, Amsterdam, 1998.

[119] YUAN, C. K., The existence of inner invariant means on $L^{\infty}(G)$. J. Math. Anal. Appl. 130 (1988), no. 2, 514-524.

[120] ZOBEIDI, A., Every topologically amenable locally compact quantum group is amenable. Bull. Aust. Math. Soc. 87 (2013), no. 1, 149-151. 


\section{Index}

$C^{*}$-algebra, 10

$I I_{1}$-factor, 15

*-representation, 13

faithful, 13

irreducible, 13

spatially nondegenerate, 13

admissible morphism, 28

analytic element, 19

antipode, 42

Arens products, 9

Arens regular Banach algebra, 9

Banach *-algebra, 9

Banach algebra, 8

Banach module, 8

bounded approximate identity, 8

character group, 94

closed quantum subgroup, 92

co-action, 134

completely bounded multiplier, 50

completely contractive Banach algebra, 25

operator amenable, 130

self-induced, 27

conditional expectation, 25

conjugate co-representation, 115

dual operator module, 26

dual quantum group, 37

exact sequence, 29

Fourier algebra, 16

Fourier-Stieltjes algebra, 17

fundamental unitary, 36

group algebra, 11
Haar measure, 6

Hopf-von Neumann algebra, 30

injective von Neumann algebra, 25

intrinsic group, 94

Kac algebra, 43

left Haar weight, 31

left invariant mean

extreme, 68

locally compact group, 6

locally compact quantum group, 34

left regular representation

locally compact group, 15

locally compact quantum group, 36

left uniformly continuous function, 11

locally compact group, 6

amenable, 7

IN, 108

inner amenable, 107

SIN, 108

locally compact quantum group, 31

amenable, 34

co-amenable, 34

co-commutative, 31

commutative, 31

compact, 34

discrete, 34

IN, 118

inner amenable, 115

regular, 103

SIN, 123

strongly inner amenable, 115

mapping

complete quotient, 21 
completely bounded, 21

completely contractive, 21

completely isometric, 21

completely positive, 21

covariant, 65

exact complete quotient, 21

normal, 15

measure algebra, 11

minimal tensor product, 14

modular automorphism group, 19

modular function, 6

module tensor product, 26

multiplicative domain, 22

multiplier algebra, 45

nuclear $C^{*}$-algebra, 25

operator module, 26

biflat, 127

biprojective, 132

essential, 26

faithful, 26

flat, 28

induced, 27

injective, 28

projective, 27

relatively biflat, 127

relatively biprojective, 132

relatively injective, 28

relatively projective, 27

operator space, 21

pentagonal relation, 36

positive

element, 12

map, 12

predual of a von Neumann algebra, 15

reduced $C^{*}$-algebra

locally compact group, 16

locally compact quantum group, 39

right Haar weight, 31

right regular representation

locally compact group, 16 locally compact quantum group, 37

right uniformly continuous function, 12

scaling group, 42

state, 12

faithful, 12

pure, 12

tracial, 12

strict topology, 45

topological group, 6

trace-class operators, 10

unitary antipode, 42

extended, 55

unitary co-representation, 47

unitization, 8

universal $C^{*}$-algebra

locally compact group, 13

locally compact quantum group, 44

von Neumann algebra, 14

weight, 17 